\title{
WestVirginiaUniversity
}

THE RESEARCH REPOSITORY @ WVU

Graduate Theses, Dissertations, and Problem Reports

2012

\section{Development of a Software Tool to Estimate Airfoil Feature Variations}

Prasheel Chaganti

West Virginia University

Follow this and additional works at: https://researchrepository.wvu.edu/etd

\section{Recommended Citation}

Chaganti, Prasheel, "Development of a Software Tool to Estimate Airfoil Feature Variations" (2012).

Graduate Theses, Dissertations, and Problem Reports. 228.

https://researchrepository.wvu.edu/etd/228

This Thesis is protected by copyright and/or related rights. It has been brought to you by the The Research Repository @ WVU with permission from the rights-holder(s). You are free to use this Thesis in any way that is permitted by the copyright and related rights legislation that applies to your use. For other uses you must obtain permission from the rights-holder(s) directly, unless additional rights are indicated by a Creative Commons license in the record and/ or on the work itself. This Thesis has been accepted for inclusion in WVU Graduate Theses, Dissertations, and Problem Reports collection by an authorized administrator of The Research Repository @ WVU. For more information, please contact researchrepository@mail.wvu.edu. 


\title{
Development of a Software Tool to Estimate Airfoil Feature Variations
}

\author{
Prasheel Chaganti
}

Thesis submitted to the Benjamin M. Statler College of Engineering and Mineral Resources at West Virginia University in partial fulfillment of the requirements for the degree of

\author{
Master of Science \\ in \\ Industrial Engineering \\ Dr. Rashpal S. Ahluwalia, Chair \\ Dr. Majid Jaridi \\ Mr. Donald J. Scott
}

Morgantown, West Virginia

December 2012

Keywords: Airfoil Manufacturing; Compressor blade; Software tool; CMM Inspection 


\section{ABSTRACT \\ Development of a Software Tool to Estimate Airfoil Feature Variations \\ Prasheel Chaganti}

The objective of this thesis is to design and develop a software tool that analyzes the incoming raw material inspection data obtained from a Coordinate Measuring Machine (CMM) and estimates feature variation created within the manufacturing process i.e. from the raw material stage to finished stage. This tool is used not only to disposition whether a lot is conforming or non-conforming, but also to provide the root installation operators an ideal $\mathrm{N}$-angle, Leading Edge Angle (LEA) and Trailing Edge Angle (TEA) target that maximize the yield of the lot after further processing. The tool also helps reduce the number of airfoil sections which need to be inspected both at In-Process and Final CMM inspection stages, thereby saving a considerable amount of inspection time as well as providing estimated cost savings of over a million dollars a year to the business. 


\section{ACKNOWLEDGEMENT}

I would like to acknowledge, first and foremost, Dr. Ahluwalia for his unwavering support throughout the degree program. He was very understanding, helpful and extremely patient when I was going through the toughest phases of my life in losing my mother a year after I started my master’s program.

My sincere thanks to Dr. Majid Jaridi for serving as a member of my thesis committee; I would also like to sincerely thank the Industrial and Management Systems Engineering department for giving me the opportunity to study at this prestigious university. I am forever indebted to each and everyone who helped me throughout my master's.

I would also like to thank Mr. James Dalton; he was a great mentor throughout my time in the mechanical lab, his dedication, hard work, relentless pursuit for safety is contagious.

I would like to acknowledge Mr. Donald Scott for his help throughout this project. I would also like to thank my director of technical services Mr. Patrick Markham for allowing me to work and present this project as my thesis.

I would like to thank Mr. Richard Zappulla II for his immense help working on MATLAB programming; I thoroughly enjoyed working with him. He is probably the smartest person I have ever met in my life.

I would like to thank my family members, my father Mr. Raghava Rao Chaganti, for his immense sacrifices to fund my studies. Of course my brother Prasanth and my sister iii 
Geetha, for not letting me give up on my master's, and for their unconditional love and support.

I would like to dedicate this thesis to my beloved mother (Late) Mrs. Sujatha Chaganti. She was the biggest inspiration in my life; she always preached that through hard work one can achieve anything. I hope that I made her proud. I love you mom and I really miss you.

Last but not the least to my lovely wife Mrs. Caitlin Murphy Chaganti, for her immense support and help when I really needed to focus and work on my master's. Thank you for being there for me; without you none of this would be possible. You are my great source of strength and inspiration. I love you. 


\section{TABLE OF CONTENTS}

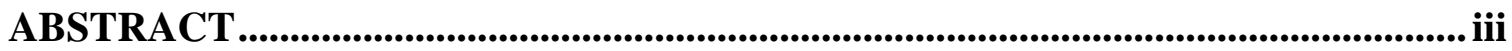

ACKNOWLEDGEMENT......................................................................................................ii

TABLE OF CONTENTS ......................................................................................................... v

LIST OF FIGURES ................................................................................................................. viii

LIST OF TABLES ….......................................................................................................

LIST OF NOTATIONS..................................................................................................... xii

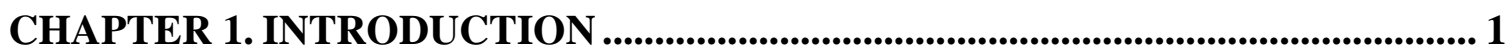

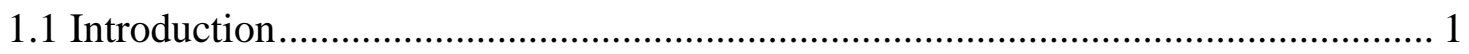

1.2 Background ................................................................................................ 2

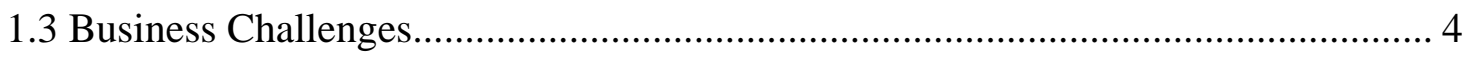

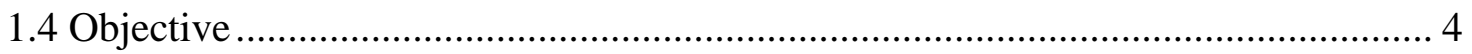

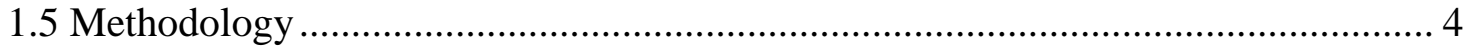

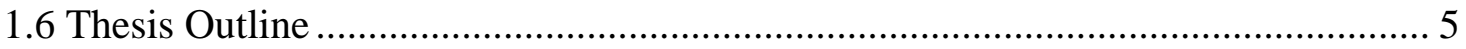

CHAPTER 2. COMPRESSOR BLADE GEOMETRY ............................................... 7

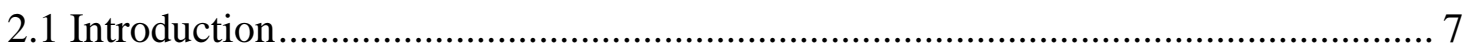

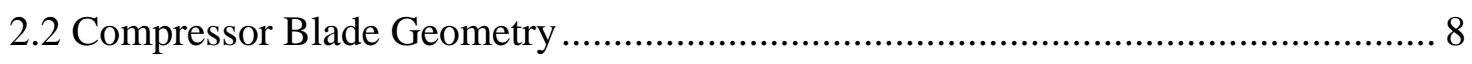

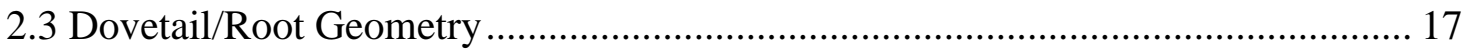

2.4 Aircraft Engines / Part families............................................................................. 17

CHAPTER 3. COMPRESSOR BLADE MANUFACTURING PROCESS............... 19

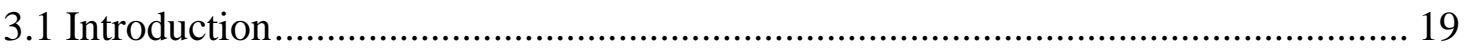

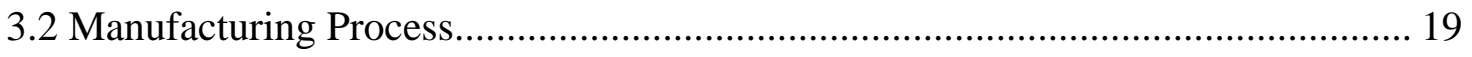

CHAPTER 4. COMPRESSOR BLADE INSPECTION ............................................... 29

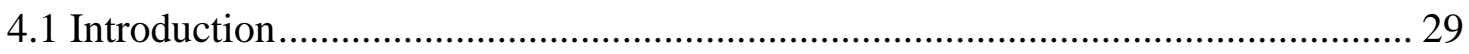

4.2 Coordinate Measuring Machine........................................................................... 29 


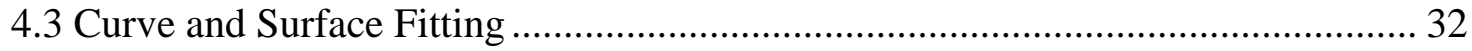

4.4 Airfoil Data Processing (PC-DMIS Blade)....................................................... 32

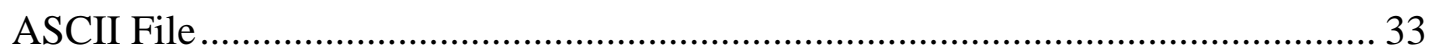

CHAPTER 5. SOFTWARE TOOL .................................................................... 36

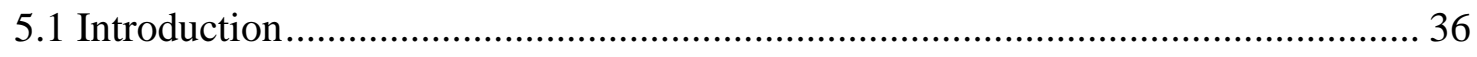

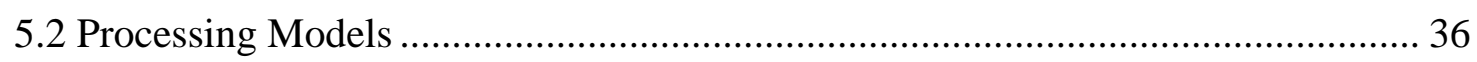

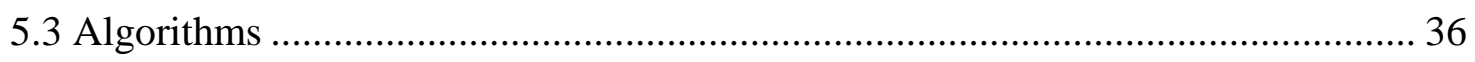

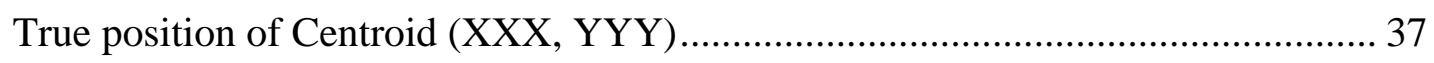

Delta True Position (DTPXXX, DTPYYY, DTPN), Adjacent Section Deviation

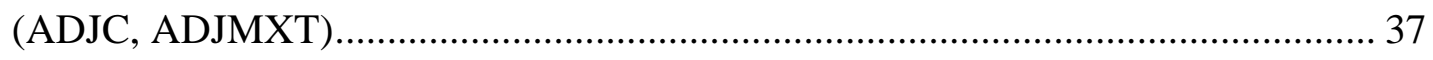

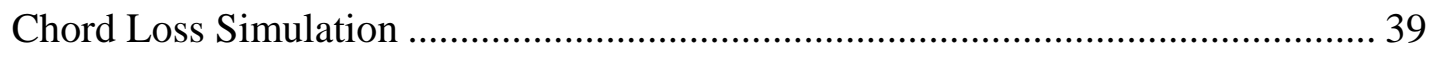

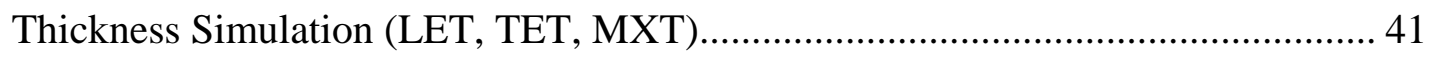

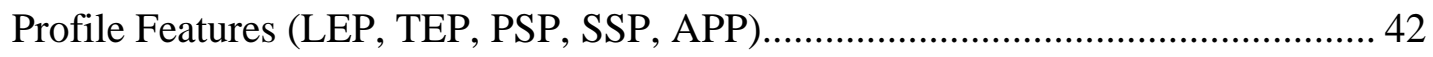

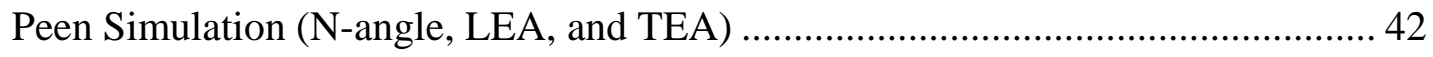

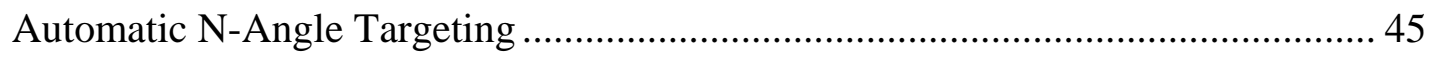

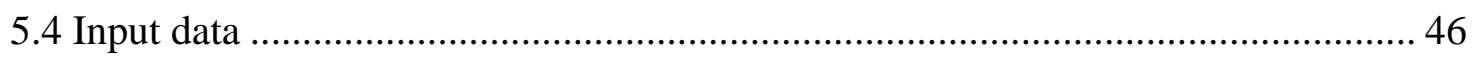

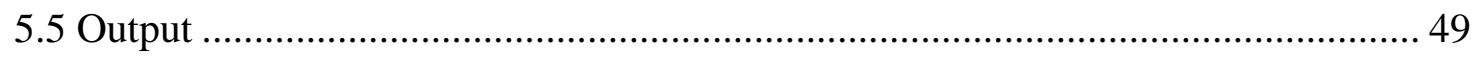

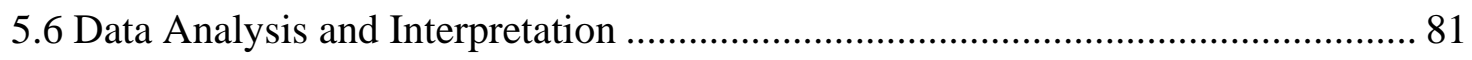

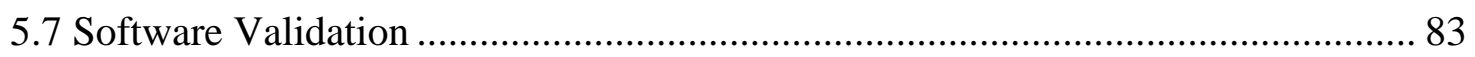

CHAPTER 6. CONCLUSION AND FUTURE WORK....................................... 86

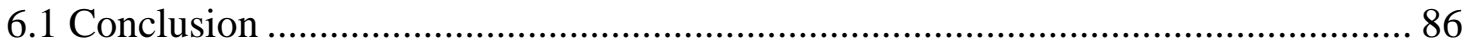

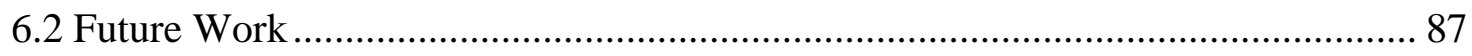

REFERENCES......................................................................................................................8 88

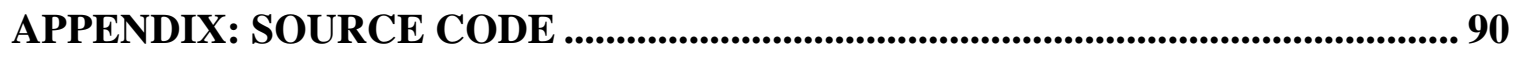

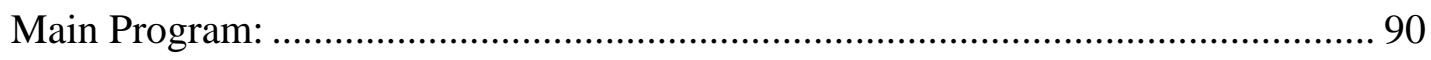


Standard Deviation \& Average Calculations:

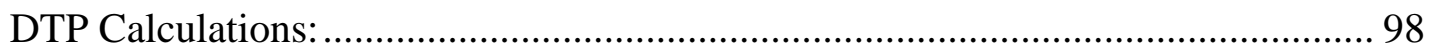

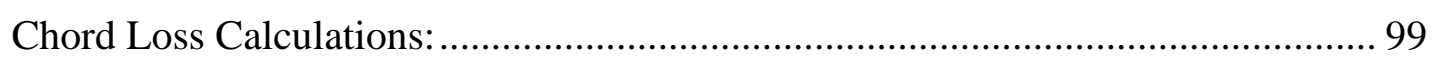

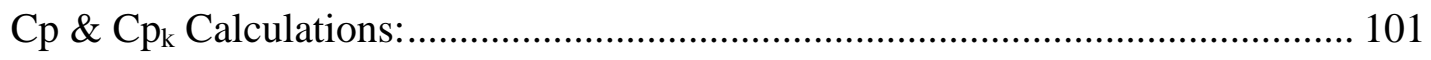

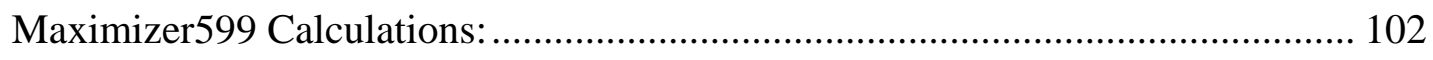

Minimum Cpk Calculations:.............................................................................. 104

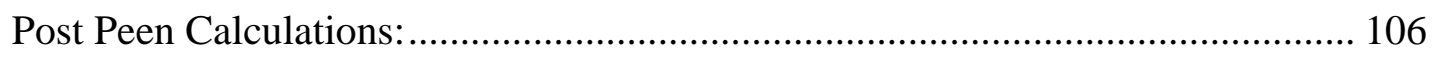

Spreadsheet Maker Calculations:_.............................................................................. 107

Normal Plot Calculations:.................................................................................. 111

Post Peen Plot Calculations: ................................................................................. 115

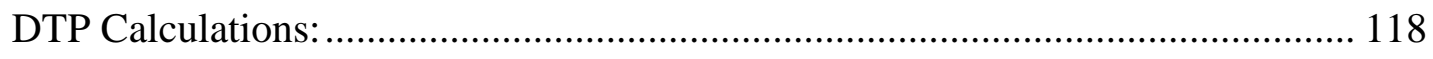

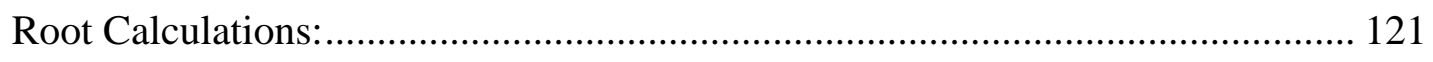

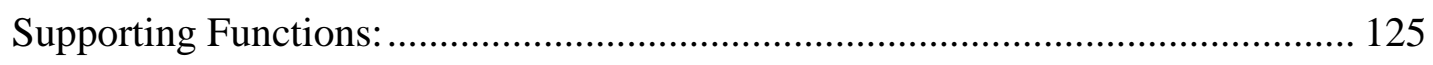




\section{LIST OF FIGURES}

Figure 1-1: A typical Compressor Blade ……………...................................................... 3

Figure 2-1: Axial Flow Jet Engine [11] ..................................................................... 7

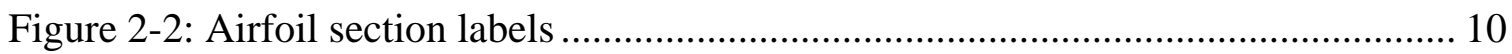

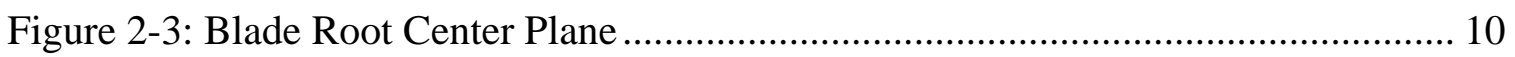

Figure 2-4: Section Label and Z-gage ..................................................................... 11

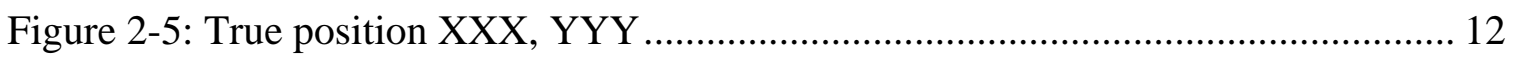

Figure 2-7: Leading, Trailing, and Maximum Thickness ................................................ 13

Figure 2-8: N-angle Deviation ......................................................................................... 14

Figure 2-9: Leading and Trailing Edge Angle.............................................................. 15

Figure 2-10: All-Around Profile ……………………………………………………..... 15

Figure 2-11: Pressure and Suction Side Profile ................................................................ 16

Figure 2-12: Leading Edge and Tailing Edge Profile....................................................... 16

Figure 2-13: Typical Compressor Blade...................................................................... 17

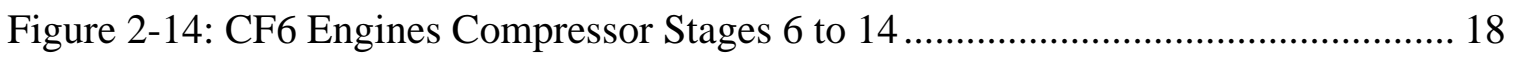

Figure 3-1: Typical forging with near net finish airfoil.................................................... 20

Figure 3-2: Encapsulation Fixture \& Encapsulated Part..................................................... 21

Figure 3-3: Forging (green) and Finished part (metallic) overlap view ............................ 23

Figure 3-4: ECG tip ground part before and after............................................................ 24

Figure 3-5: Penetrant application \& dwell, crack readout under a black light[21][22].... 26

Figure 3-6: Shot peening dimple, compressive layer after shot peening ........................... 27

Figure 3-7: Different shapes \& sizes of media, ceramic \& plastic media ......................... 28

Figure 4-1: System Components of a CMM [24] .......................................................... 31

Figure 4-2: Sample ASCII file for a section of airfoil ...................................................... 34 
Figure 4-3: Airfoil Section Definition by Points and Local Normals............................ 35

Figure 5-1: Airfoil Section Centroid Deviation Differences ........................................ 39

Figure 5-2: Visual representation fo post peen Cpk vs Offset ....................................... 46

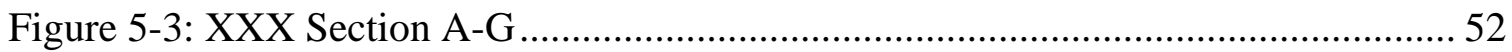

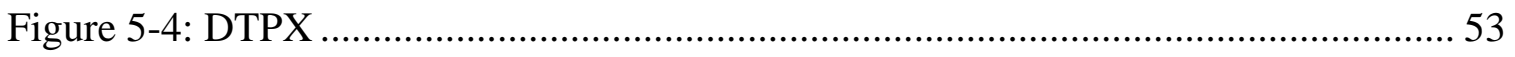

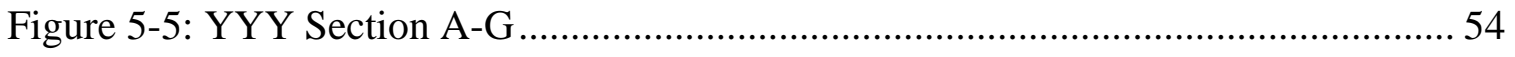

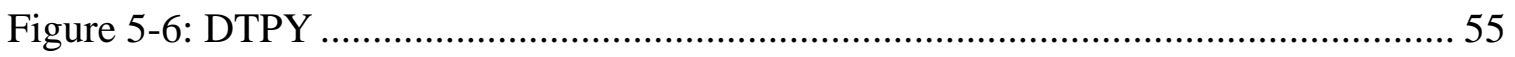

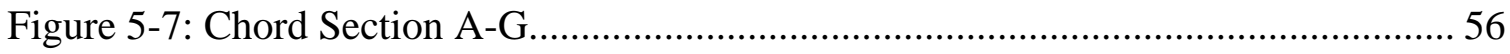

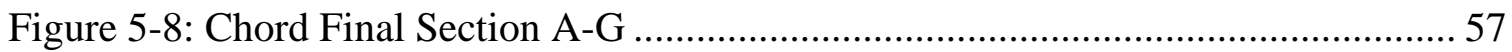

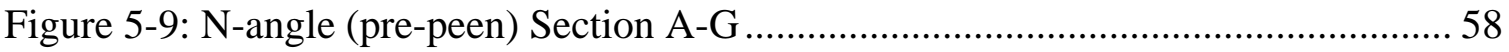

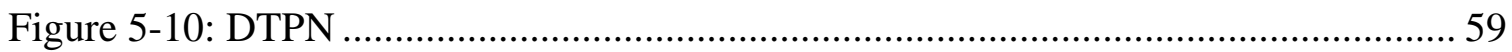

Figure 5-11: Leading Edge Angle (pre-peen) Section A-G........................................ 60

Figure 5-12: CLEA (Camber LEA)- Information only........................................... 61

Figure 5-13: Trailing Edge Angle (pre-peen) Section A-G ......................................... 62

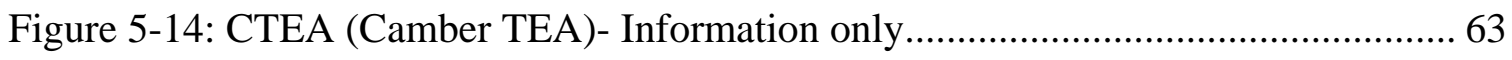

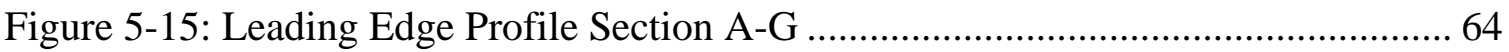

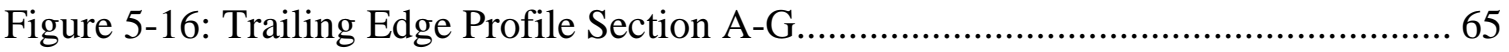

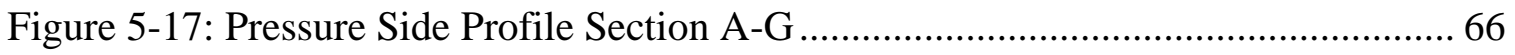

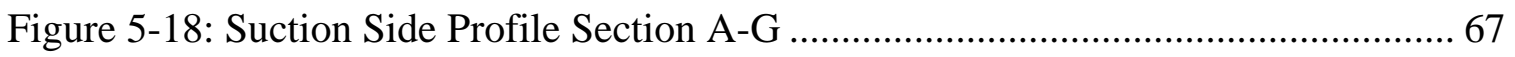

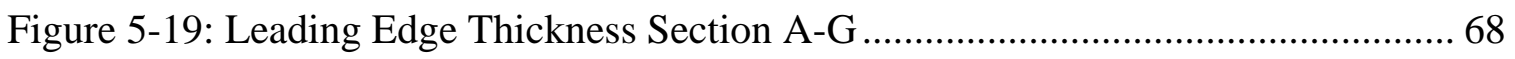

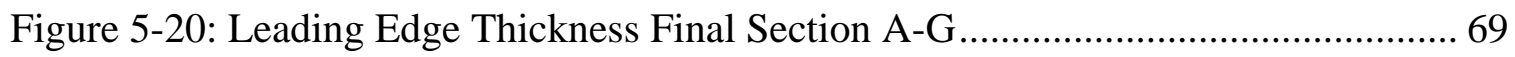

Figure 5-21: Trailing Edge Thickness Section A-G ................................................ 70

Figure 5-22: Trailing Edge Thickness Final Section A-G ........................................... 71

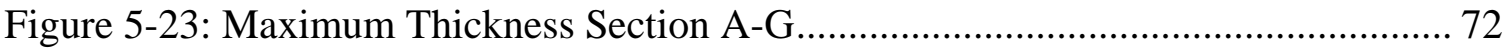


Figure 5-24: Maximum Thickness Final Section A-G ............................................. 73

Figure 5-25: N-angle (post-peen) Section A-G with N-angle target offset ..................... 74

Figure 5-26: Leading Edge Angle (post-peen) Section A-G with N-angle target ............ 75

Figure 5-27: Trailing Edge Angle (post-peen) Section A-G with N-angle target ............ 76

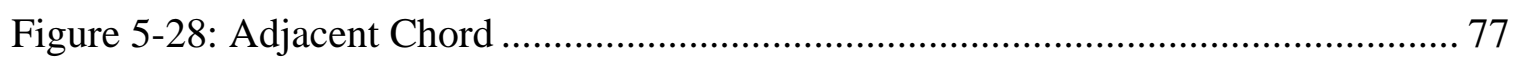

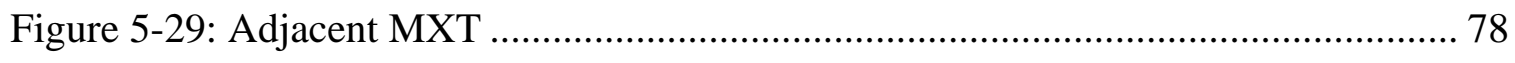

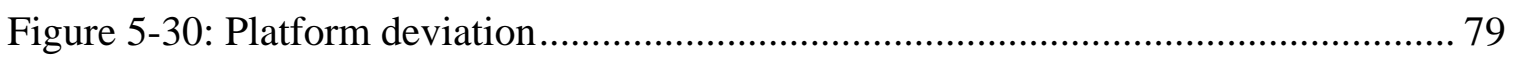

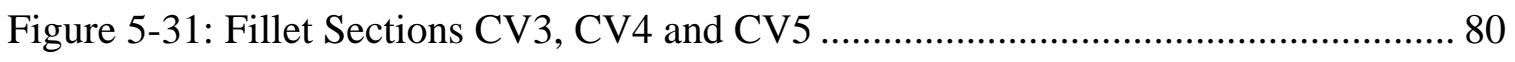

Figure 5-32: IMS sheet for N-Angle target and CMM reduced section inspection........... 82 


\section{LIST OF TABLES}

Table 5-1: Centroid deviation per section......................................................................... 38

Table 5-2: Centroid deviation calculations ......................................................................... 38

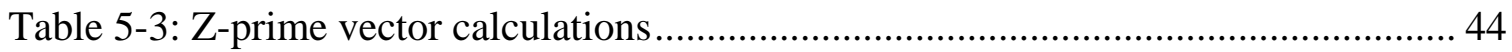

Table 5-4: Airfoil Inspection Data ................................................................................ 47

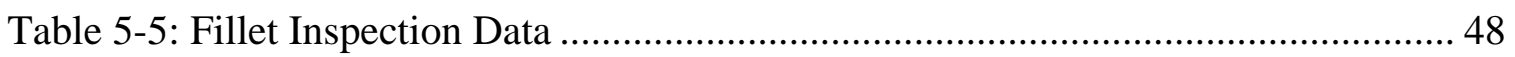

Table 5-6: Platform Inspection Data................................................................................ 48

Table 5-7: Raw data forging to final calculations........................................................... 50

Table 5-8: $\mathrm{C}_{\mathrm{p}}$ and $\mathrm{C}_{\mathrm{pk}}$ calculations at IP (In-Process) ………………………………..... 51

Table 5-9: $\mathrm{C}_{\mathrm{pk}}$ at final processing................................................................................ 51

Table 5-10: Calculated feature output from the software tool............................................ 84

Table 5-11: Actual feature output of manufactured lot (CMM Final Inspection) ............. 84

Table 5-12: Difference between calculated and actual feature output............................... 84

Table 5-13: Cost saving before and after tool implementation.......................................... 85 


\section{LIST OF NOTATIONS}

\begin{tabular}{|c|c|}
\hline $\mathrm{TE}$ & Trailing Edge \\
\hline LE & Leading Edge \\
\hline CC & Concave Side \\
\hline $\mathrm{CV}$ & Convex Side \\
\hline $\mathrm{C}$ & Chord Length \\
\hline $\mathrm{C}_{\mathrm{F}}$ & Chord Length Final \\
\hline $\mathrm{C}_{\mathrm{L}}$ & Chord Loss \\
\hline LET & Leading Edge Thickness \\
\hline $\mathrm{LET}_{\mathrm{F}}$ & Leading Edge Thickness Final \\
\hline TET & Trailing Edge Thickness \\
\hline $\mathrm{TET}_{\mathrm{F}}$ & Trailing Edge Thickness Final \\
\hline MXT & Maximum Thickness \\
\hline $\mathrm{MXT}_{\mathrm{F}}$ & Maximum Thickness Final \\
\hline $\mathrm{N}, \mathrm{N}_{1}$ & N-Angle \\
\hline LEA, LEA 1 & Leading Edge Angle \\
\hline CLEA & Camber Leading Edge Angle \\
\hline CTEA & Camber Trailing Edge Angle \\
\hline $\mathrm{TEA} \mathrm{TEA}_{1}$ & Trailing Edge Angle \\
\hline $\mathrm{Pn}_{1}$ & Post-peen N-angle \\
\hline Plea $_{1}$ & Post-peen Leading Edge Angle \\
\hline Ptea $_{1}$ & Post-peen Trailing Edge Angle \\
\hline AAP & All Around Profile \\
\hline PSP & Pressure Side Profile \\
\hline SSP & Suction Side Profile \\
\hline LEP & Leading Edge Profile \\
\hline TEP & Trailing Edge Profile \\
\hline CMM & Coordinate Measuring Machine \\
\hline FAA & Federal Aviation Administration \\
\hline OEM & Original Equipment Manufacturer \\
\hline
\end{tabular}




\begin{tabular}{|l|l|}
\hline PMA & Part Manufacturing Approval \\
\hline NDT & Non-Destructive Testing \\
\hline BRCP & Blade Root Center Plane \\
\hline ECG & Electro Chemical Grinding \\
\hline CAD & Computer Aided Design \\
\hline FPI & Florescent Penetrant Inspection \\
\hline SPC & Statistical Process Control \\
\hline UCL & Upper Control Limit \\
\hline LCL & Lower Control Limit \\
\hline USL & Upper Specification Limit \\
\hline LSL & Lower Specification Limit \\
\hline IMS & Inspection Method Sheet \\
\hline MCL & Mean Camber Line \\
\hline
\end{tabular}




\section{CHAPTER 1. INTRODUCTION}

\subsection{Introduction}

Two of the inventions that have greatly shaped our modern day lives are the invention of the computer and the invention of the fixed wing aircraft [1]. Both of these have come a long way since their inception. Computers [2], for instance, are used in nearly every facet of our lives from smallest microchips to the largest servers. Modern day computers are put to use in every major industry. They power our healthcare industry, aid in supplying energy to our homes, and drive most elements in our manufacturing facilities. In manufacturing, computers have taken the production of aircraft components to a whole new level. The computer's impact on component design, prototyping, test simulation etc made manufacturing of these modern day aircraft possible. Without computers, airplanes, as we know it, would not exist.

The impact of an aircraft on our modern world is felt in many aspects of our lives; the products and services that were never available are at our finger tips today, the exotic foods that we eat, the medication we use, the life saving organ transplants, the manner in which we go to wars, national surveillance, etc. The accessibility of air travel on an international level has changed the way we do business, taking local and regional markets to a global stage. Both the computer and the fixed wing aircraft have had a critical impact on the development and globalization of our modern society [3].

The jet engine [4] is one of the most critical components of an aircraft. A typical jet engine has a fan, compressor, combustor, turbine and an exhaust system. It is imperative to understand the workings of a jet engine in order to know compressor blade design. Essentially the engine sucks the air in at the front of the engine through a fan, and the air flows into the compressor section where it is compressed thus 
raising the pressure. This compressed air is then mixed with fuel, and an electric spark ignites the mixture. The burning gas expands in the turbine section and blasts through the exhaust system or nozzle at the back of the engine. Since the working fluid passes through the engine parallel to the axis of rotation of the engine, these engines are known as axial flow engines [5].

\subsection{Background}

The airfoil is a very common shape found in nature; the most obvious ones are the wings of a bird, the fins of a fish etc. Each airfoil shape has a distinct character, and they vary by shape and sizes depending on the function of that airfoil. The most notable airfoils are used in airplane wings, fan blades, and propellers. One such application of an airfoil is the compressor blade that is used in the high pressure and low pressure compressor sections of a jet engine. The focus of this thesis is on compressor blades [6].

\section{Forging}

"Forging is defined as the plastic deformation of metals at elevated temperature into a predetermined size or shape using compressive forces exerted through some means of hand hammers, small power hammers, die, press or upsetting machine” [7]. The metal is normally, but not always, preheated to a desired temperature before the forging operation [8]. The forging processes can be classified into hot forging and cold forging, with each classification providing its own advantages and disadvantages.

In the forging process, as the metal is pounded, the grain deformation causes an unbroken chain of grain flow following the shape of the part; this creates parts that are significantly stronger than those created from other conventional metal working processes. This advantage of a high strength-to-weight ratio is the reason why they are used in applications where human safety and reliability are critical. Some of the 
applications of the forged parts are found within items such as airplanes, automobiles, earth mowing equipment, golf equipment, missiles etc [8].

\section{Compressor Blades}

A compressor blade (also known as blade) has two main sections: Airfoil and Root (also known as dovetail), as shown in Figure 1-1 below. The root secures the airfoil to the disk; there are several disks to accommodate each stage of the compressor blade. The blade geometry is discussed more in detail in Chapter 2. Airfoils are created using a forging process to near net tolerances at a supplier. These forging lots, once received from the supplier are then inspected on a CMM (Coordinate Measuring Machine) [9]. Lot accept/reject determination is made by comparing inspection results to the design requirements. If a lot is found to be acceptable, the remainder of the manufacturing process is carried out.

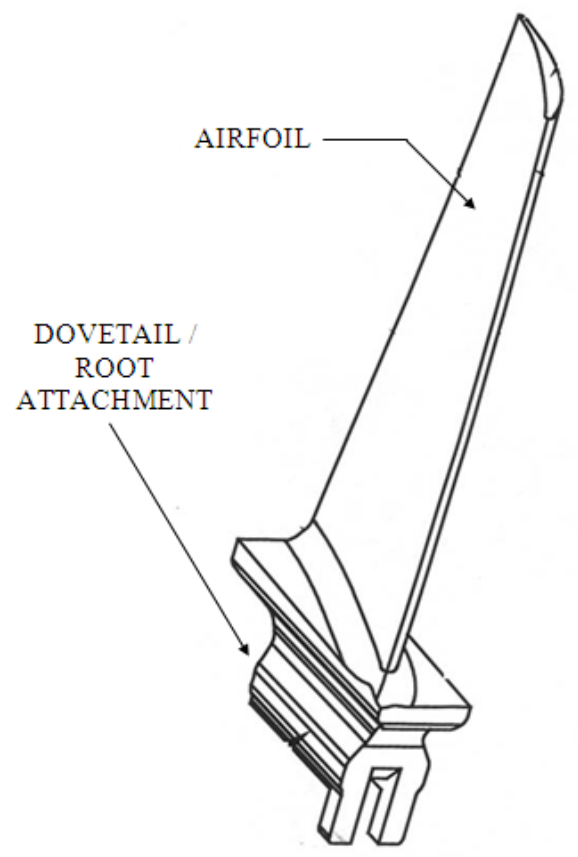

Figure 1-1: A typical Compressor Blade 


\subsection{Business Challenges}

The compressor blade is a key component of a jet engine. Due to the importance of its application and consequences of its failure causing in-flight shutdowns, the FAA (Federal Aviation Administration) has classified them as "major" parts. The complex design, in addition to the significant characterization, makes the manufacturing and inspection of the compressor blades a daunting task. Some of the dimensional tolerances that are required to be maintained are defined to ten thousands of an inch. Due to the high volume of the manufacturing and the inspection of all airfoil features, the inspection costs have increased significantly. Here in lies a serious need to reduce the inspection costs while maintaining the highest levels of quality.

\subsection{Objective}

The objective of this thesis is to design and develop a software tool that estimates airfoil feature variations throughout the manufacturing process which will help reduce the CMM inspection time and CMM inspection costs.

\subsection{Methodology}

The airfoil section of the compressor blade is forged and shipped from a supplier; the dovetail is installed and the compressor blade is processed through the remainder of the manufacturing process. Once the forgings are received they are CMM inspected; the inspection data are then analyzed to verify the dimensional accuracy of the forgings, essentially to accept or reject the forging lots before proceeding with the rest of the manufacturing process.

Because of the considerable variation inherent in the forging process, we must take it upon ourselves to capture these process effects and adjust the manufacturing process accordingly to conform to the design 
requirements. The idea is that once we understand all process effects on the features, one can accurately predict these feature variations throughout the manufacturing process thereby eliminating some of the redundant airfoil section CMM inspections which are built into the process. Hence the process effects are analyzed throroughly, and models are formulated and packaged into a software tool for simplifying the calculations to assist in lot disposition, reduced section inspection. The end result is to reduce considerable inspection time and inspections costs.

In addition to the above, an ideal $\mathrm{N}$-angle offset, which will be discussed in detail in Chapter 3, will assist the grind operator target the critical airfoil features like N-angle, LEA, TEA in relation to the root, to maximixe the yield of the manufacturing lot.

To summarize the methodology:

a) Develop a software tool that can estimate changes in airfoil features from forging to finish stage. This will help reduce the number of airfoil section inspections, therefore decreasing inspection time and inspection costs.

b) Compute the Ideal $\mathrm{N}$-angle offset target value which will potentially eliminate fallouts at final inspection, thereby increasing the yield.

c) Develop criteria to accept or reject a forging lot based on the inspection results.

\subsection{Thesis Outline}

In Chapter 1 the topic of interest is introduced to the reader and business challenges were explained which leads to a methodology that is clearly defined to set the boundaries of this thesis leading to the objective of the thesis. 
Chapter 2 gives the reader a thorough knowledge of the compressor blade features that are discussed in this thesis. This chapter also briefly discusses different jet engines and different stages of compressor blades.

Chapter 3 addresses the compressor blade manufacturing process to provide a better understanding of how the compressor blade features are affected by the manufacturing process.

Chapter 4 discusses the Coordinate Measuring Machine, the compressor blade inspection process, and understanding curve fitting to process airfoil feature data.

Chapter 5 covers the software tool development, algorithms, data input, computations and output from the tool. An example is studied which explains in detail the data analysis and interpretation of the results and also the validation of the results.

Chapter 6 deals with the conclusion and future work. 


\section{CHAPTER 2. COMPRESSOR BLADE GEOMETRY}

\subsection{Introduction}

The compressors used in the modern jet engines are the axial-flow compressor type. The axial-flow jet compressor is one in which the working fluid (air) flow enters the compressor in an axial direction (parallel with the axis of rotation) and exits from the gas turbine also in an axial direction, as shown in Figure 2-1 below. The axial-flow compressor compresses the working fluid by first accelerating the fluid and then diffusing it to obtain a pressure increase. The fluid is accelerated by a row of rotating airfoils (blades) called the rotor, and then diffused in a row of stationary blades called the stator. The diffusion in the stator converts the velocity increase gained in the rotor to a pressure increase. A combination of a rotor followed by a stator makes up a stage in a compressor. A compressor consists of several stages [10].

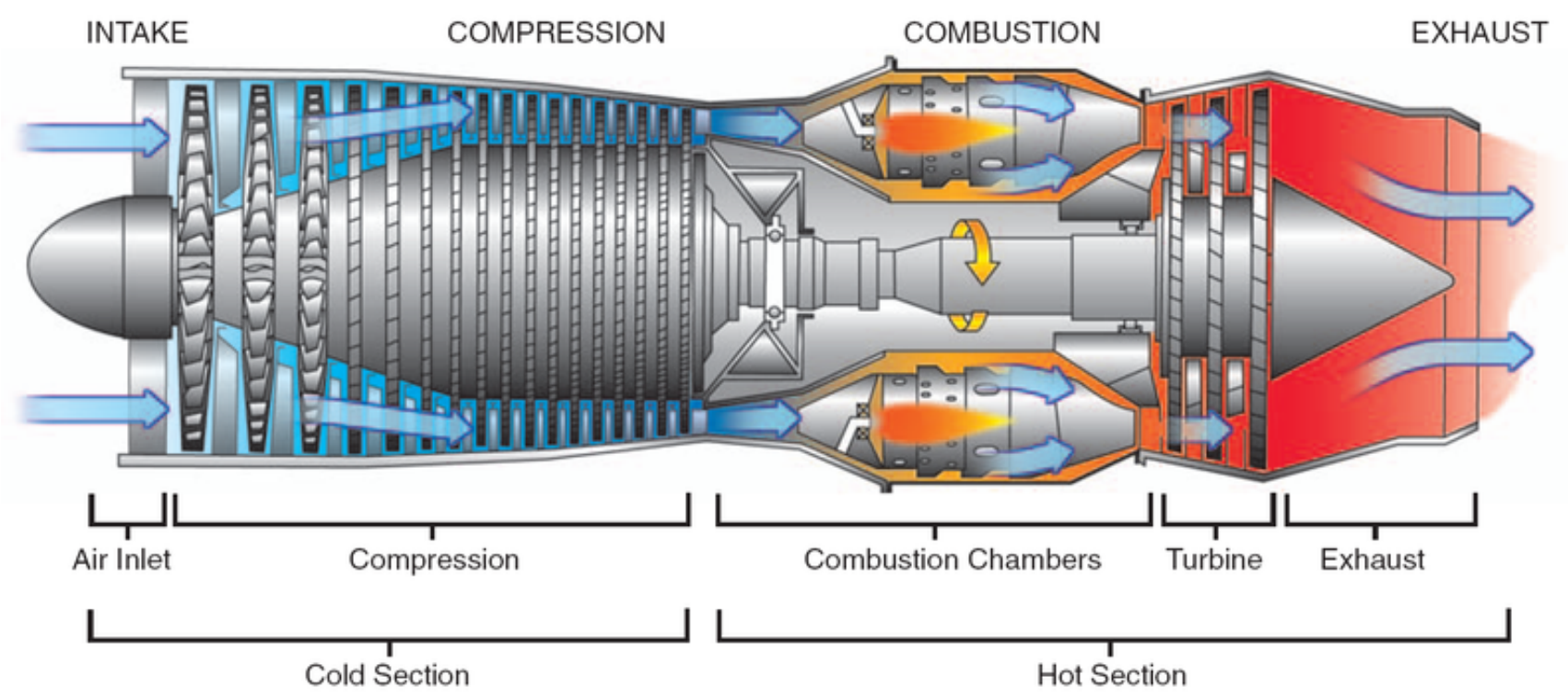

Figure 2-1: Axial Flow Jet Engine [11]

Axial flow compressors produce a continuous flow of compressed gas, and have the benefits of high efficiencies and large mass flow capacity, particularly in relation to their cross-section. They do, 
however, require several rows of airfoils to achieve large pressure rises making them complex and expensive relative to other designs [12].

\subsection{Compressor Blade Geometry}

All gas turbine propulsion systems must have a compressor component that develops some or all the pressure increase specified by the system design cycle. Shaft for the compression process is supplied by the turbine component of the system. In a modern jet engine, the compressor unit is typically divided into two sections: the low-pressure compressor and high-pressure compressor. Compressor blades designs are drastically different from engine to engine as they depend on the design characteristics that change with each stage within a jet engine. It is rather interesting to note that these compressor airfoils would exhibit some of the same behavioral characteristics that you would see in isolated airfoils (wings, etc). For example, they are subjected to lift and drag forces, they stall, and they generate boundary layers, wakes and under certain circumstances shock waves. However, compressor blades operate under conditions unlike typical isolated airfoils [13].

\section{Airfoil Geometry}

Typical compressor blade geometry is shown in Figure 2-1. It consists of four main segments: airfoil, airfoil fillet, platform and root also known as dovetail due to its shape. An airfoil is an aerodynamic surface mounted within a flow area intended to redirect the working fluid with that area. An airfoil's pressure side is the concave surface of the airfoil, while an airfoil's suction side is the convex surface of the airfoil. The airfoil's leading edge is the forward facing edge surface of the airfoil, and the trailing edge is the aft edge surface of the airfoil [14]. 


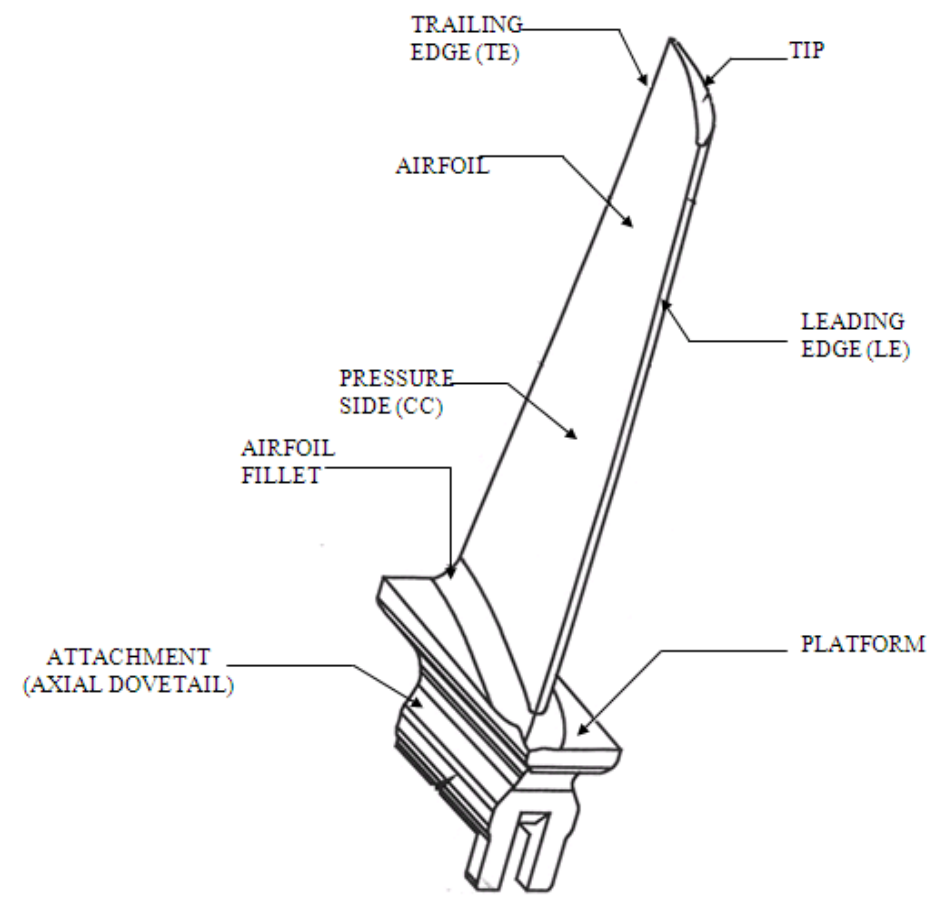

Figure 2-1: Typical Geometry of a Compressor Blade

It is a common practice in the industry is to divide the airfoil up into sections usually denoted by letters A, B, C... etc., depending on how long the airfoil is, as shown in Figure 2-2. The sections are at a known distance from a set datum scheme and all airfoil features are inspected at each specified section using the CMM machines and they are compared to the design model for any deviations. Described below are compressor blade features.

Mean Camber Line (MCL) is a line generated from the midpoints between suction side (convex side or CV side) and pressure side (concave side or CC side) profiles. 


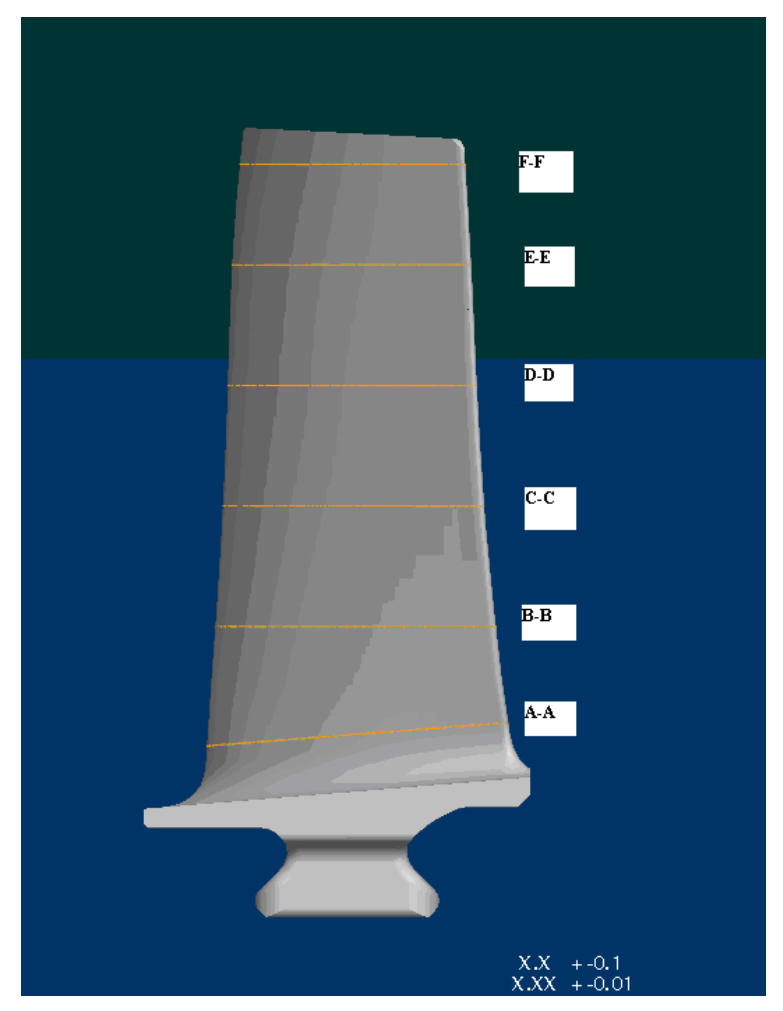

Figure 2-2: Airfoil section labels

Figure 2-3 shows the Blade Root Center Plane (BRCP) view, located at the longitudinal symmetrical center of the dovetail/root attachment.

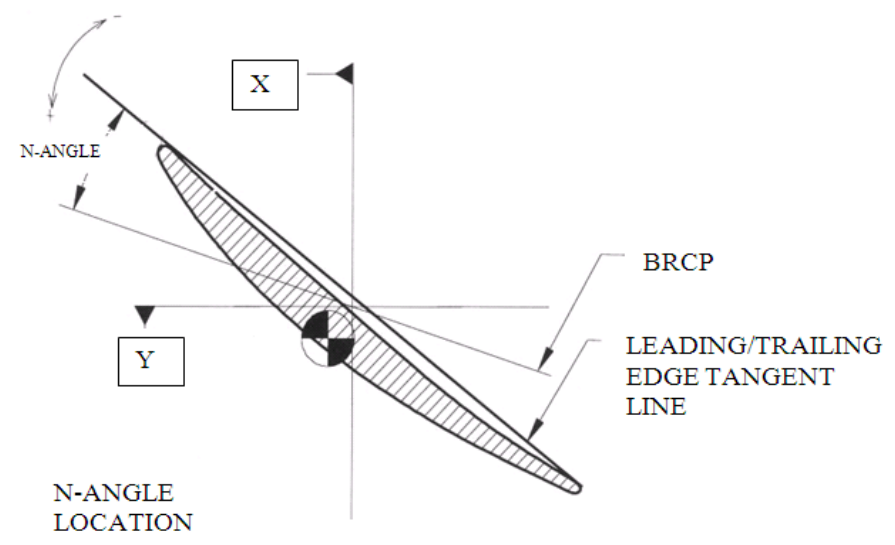

Figure 2-3: Blade Root Center Plane 
Figure 2-4 shows Section Label and Z-Gage, The section label corresponds to a given cross section taken at the specified gage (basic) distance from the stacking line coordinate system defined on the part drawing. If the cross section is canted (angled) then the gage distance is the point along the stacking line where the cant angle is applied. Canted sections have only one rotation which is about the y-axis. All canted section parameters are calculated perpendicular to the stacking line at the gage distance (they are not calculated in the cant plane).

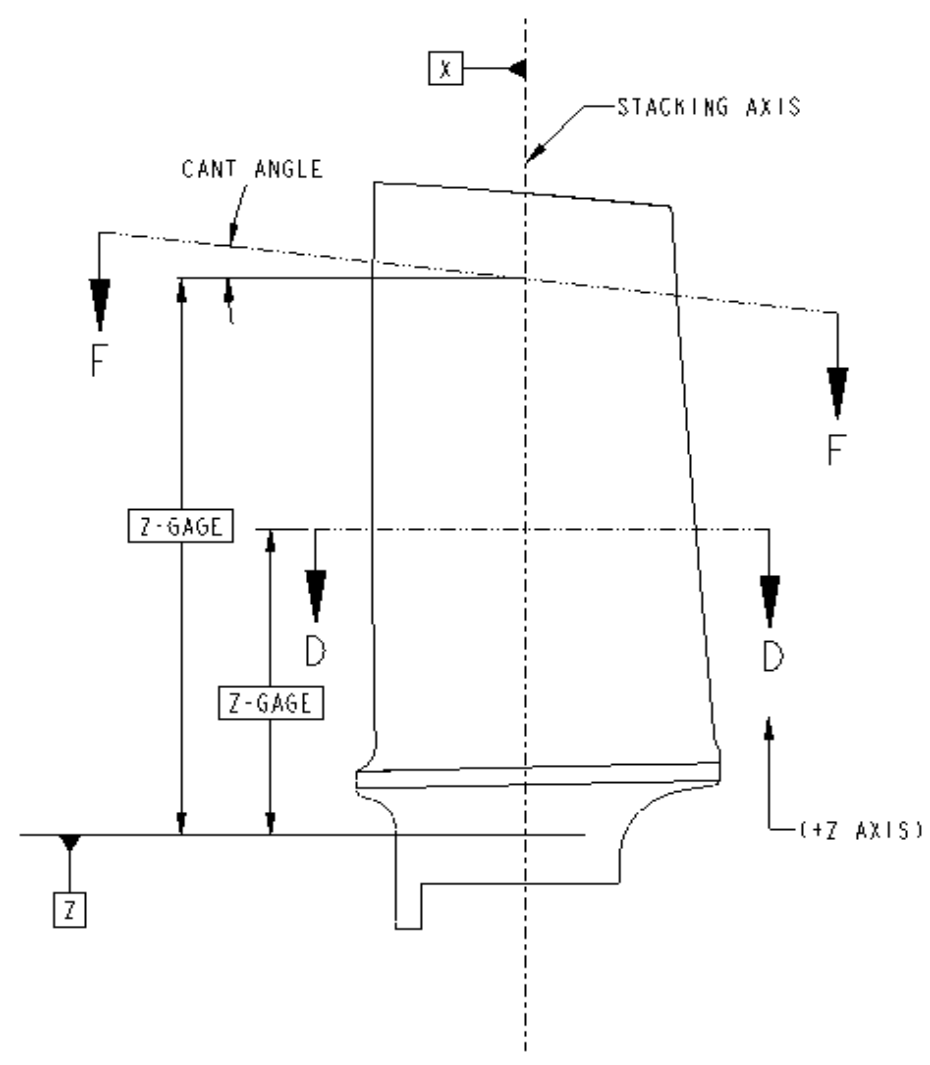

Figure 2-4: Section Label and Z-gage 
Figure 2-5 shows the True position of the centroid [XXX, YYY], measured with respect to the stacking axis. The stacking axis is the datum line normal to datum $\mathrm{Z}$, through datum $\mathrm{X}$ and $\mathrm{Y}$ and extending radially outward. The actual section centroid deviation is reported. In addition each adjacent centroid deviation difference and each $\mathrm{N}$-angle deviation difference must not exceed the drawing requirements.

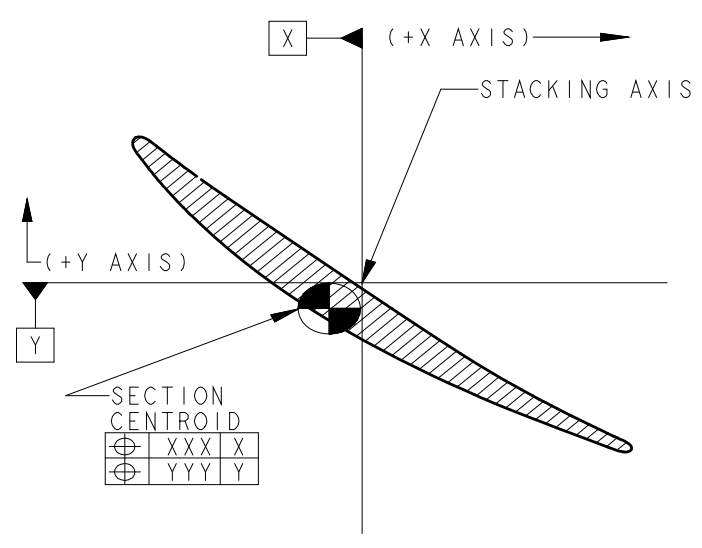

Figure 2-5: True position XXX, YYY

Figure 2-6 shows the Chord length $[\mathrm{C}]$ is defined by the maximum length of the airfoil cross-section. The chord line is the straight line passing through the Leading Edge (LE) point and Trailing Edge (TE) point.

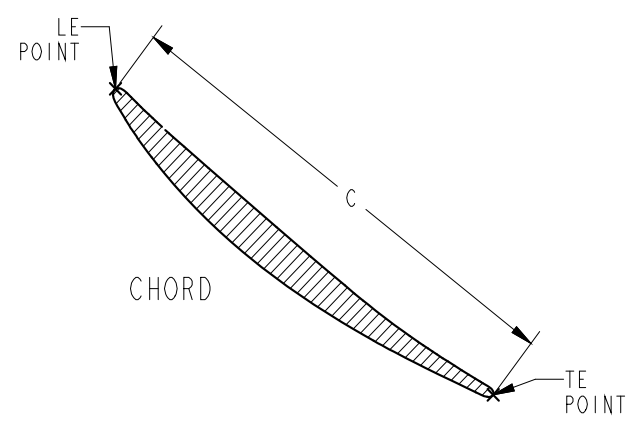

Figure 2-6: Chord Length Deviation 
The Leading Edge Thickness (LET) and Trailing Edge Thickness (TET) deviation are the thickness deviations at a basic distance from the leading and trailing edges measured along the mean camber line. The leading edge and trailing edge thickness deviation are taken at a gage (basic) distance from the LE point parallel to the mean camber line. The Maximum Thickness (MXT) deviation occurs at the thickest point along the mean camber line, as shown in the Figure 2-7

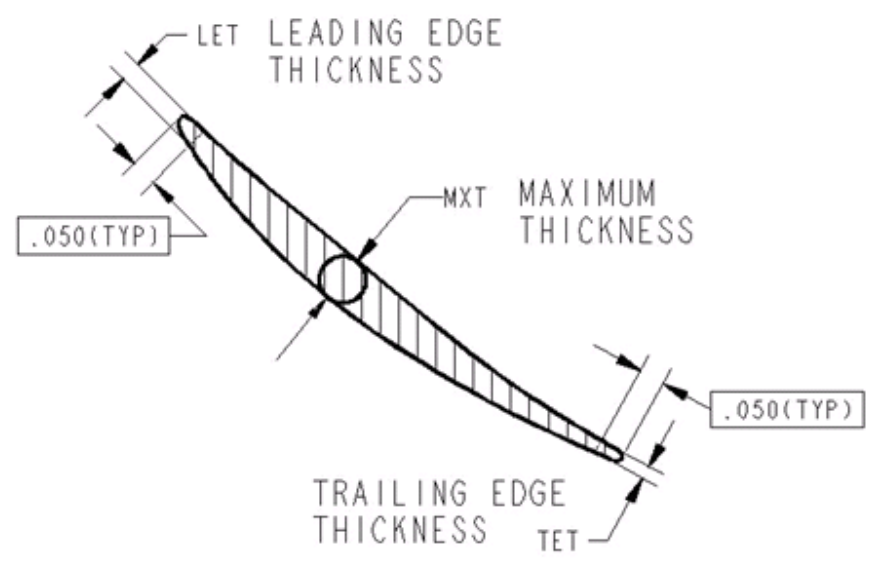

Figure 2-7: Leading, Trailing, and Maximum Thickness

The $\mathrm{N}$-angle $[\mathrm{N}]$ is an angle determined by extending a line across the tangency points of the pressure side surface of the airfoil and the applicable datum. The rotation occurs about the section centroid, as shown in the Figure 2-8. 


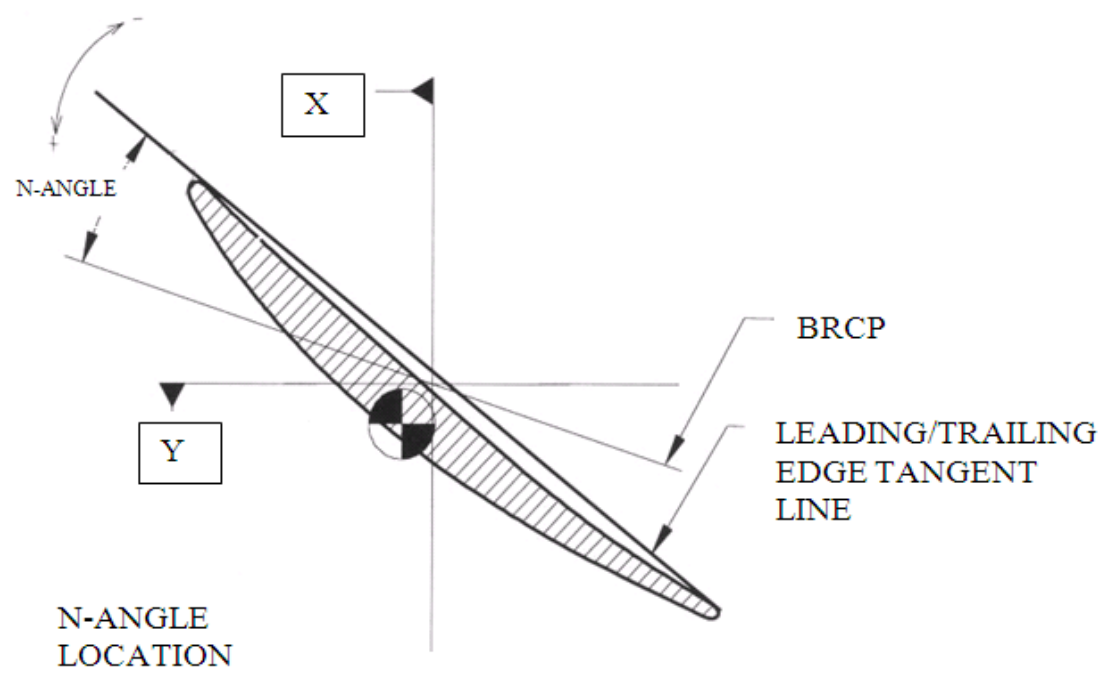

Figure 2-8: N-angle Deviation

The Leading Edge Angle [LEA] deviation is taken at a gage (basic) distance from the LE point. First the camber angle deviation is calculated by best fitting a straight line through the mean chord line between the LET gage distance and LEA gage distance and computing the deviation from the nominal. Since the camber angle measurement is taken after the section has been best fit for $\mathrm{N}$-angle deviation, the $\mathrm{N}$-angle deviation is added to the camber angle deviation resulting in leading edge angle deviation with respect to the applicable datum. Trailing Edge Angle [TEA] deviation is calculated in a similar manner as shown in Figure 2-9. 


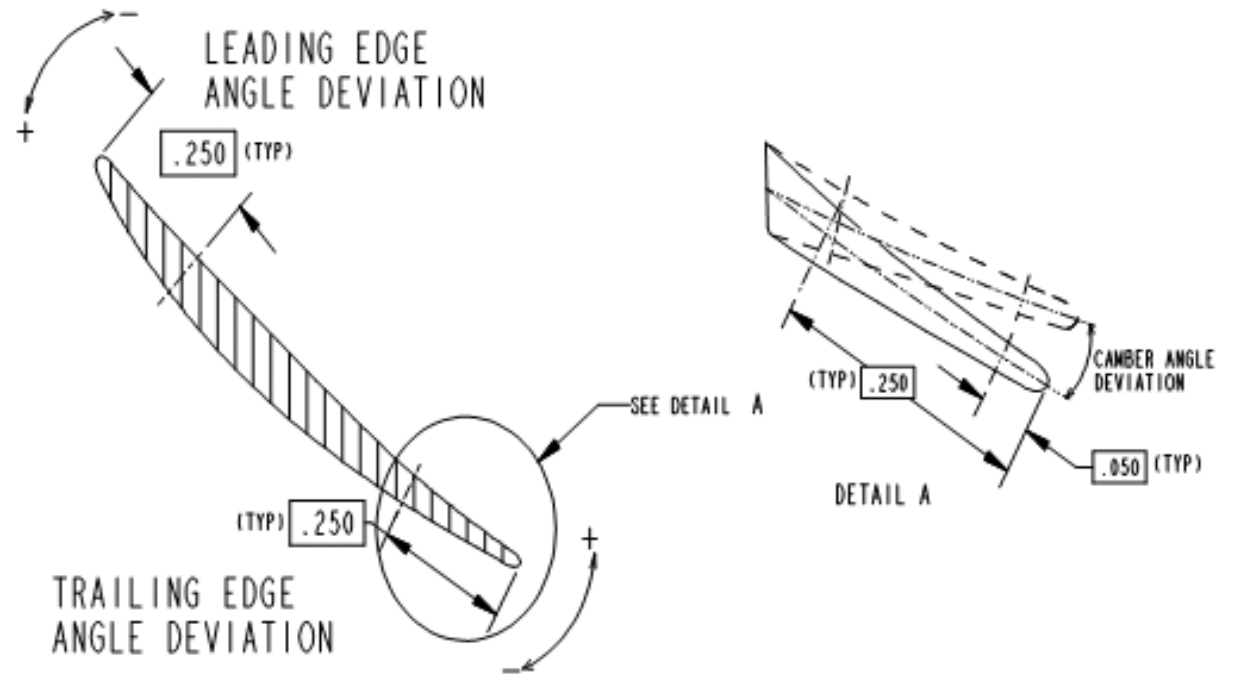

Figure 2-9: Leading and Trailing Edge Angle

The All-Around Section Profile [AAP] deviation from nominal is calculated after the best fitting of the airfoil cross section. Transitional and Rotational degrees of freedom are permitted. The allowable limits apply simultaneously around the airfoil, normal to basic airfoil. LEP, TEP points are not included in AAP, as shown in Figure 2-10 below.

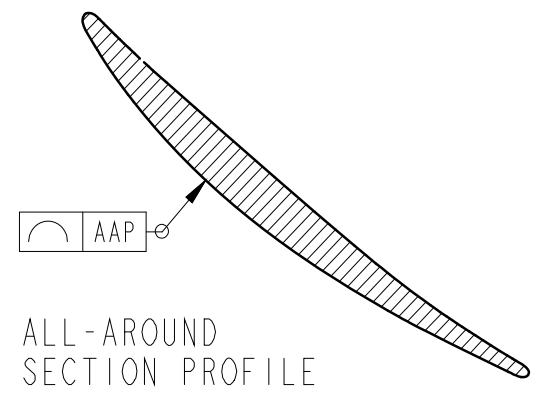

Figure 2-10: All-Around Profile 
The Pressure Side Profile [PSP] and Suction Side Profile [SSP] deviations are calculated independently after the all-around section profiles are best fit. These profile deviations generally have tighter tolerances when compares to the AAP, so the individual Pressure and Suction side contours are closely monitored for proper form, as shown in Figure 2-11.
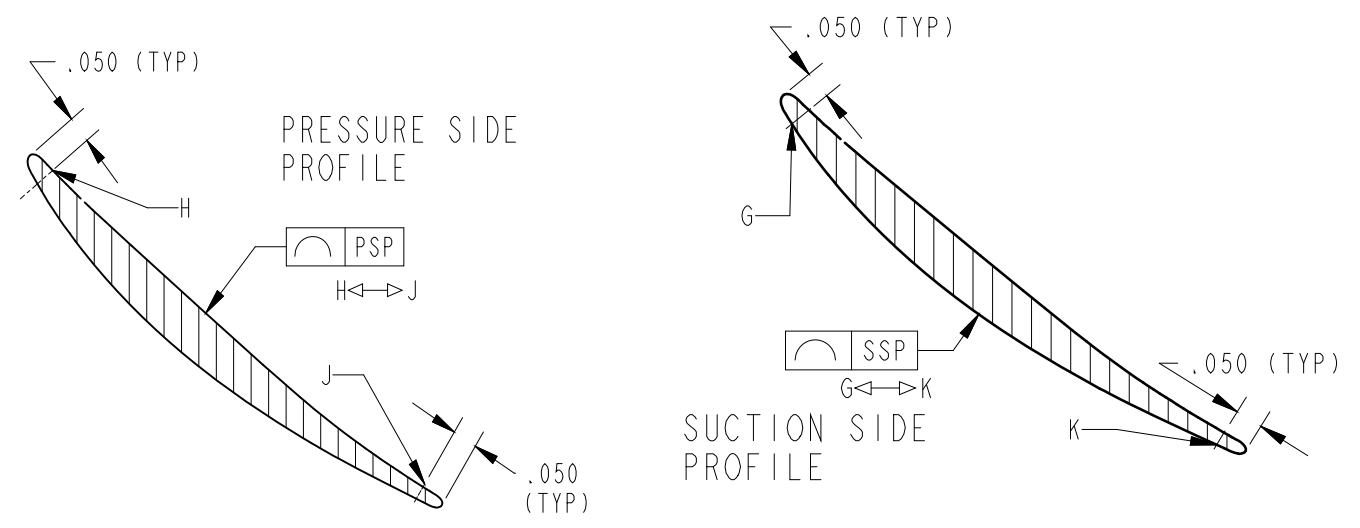

Figure 2-11: Pressure and Suction Side Profile

The Leading Edge Profile [LEP] and Trailing Edge Profile [TEP] deviations are calculated independently after the best-fits. The basic (gage) distance is measured along the mean camber line, as shown in the Figure 2-12
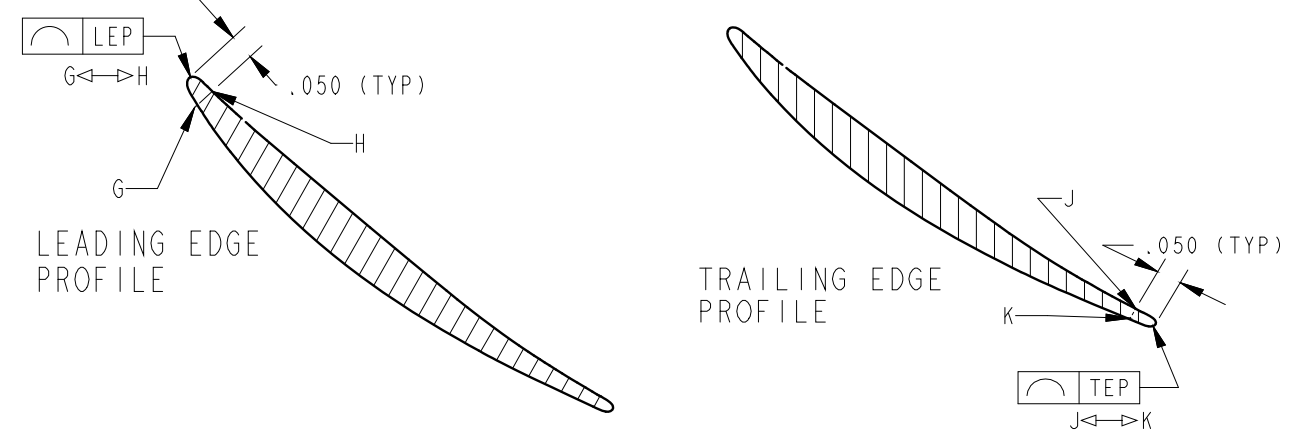

Figure 2-12: Leading Edge and Tailing Edge Profile 


\subsection{Dovetail/Root Geometry}

Dovetail is the airfoil mounting feature located at the base of the airfoil. It is typically an axial dovetail, a tangential dovetail, or a pinned root. The dovetail is what secures the airfoil to the rotor and keeps it in desired location. Platform is a mounting plate which provides transition from the airfoil fillet(s) to attachment (dovetail) features. Fillet is the transition radius between the airfoil and the platform. As shown in Figure 2-13 below, a typical compressor blade geometry used in modern axial flow engines.

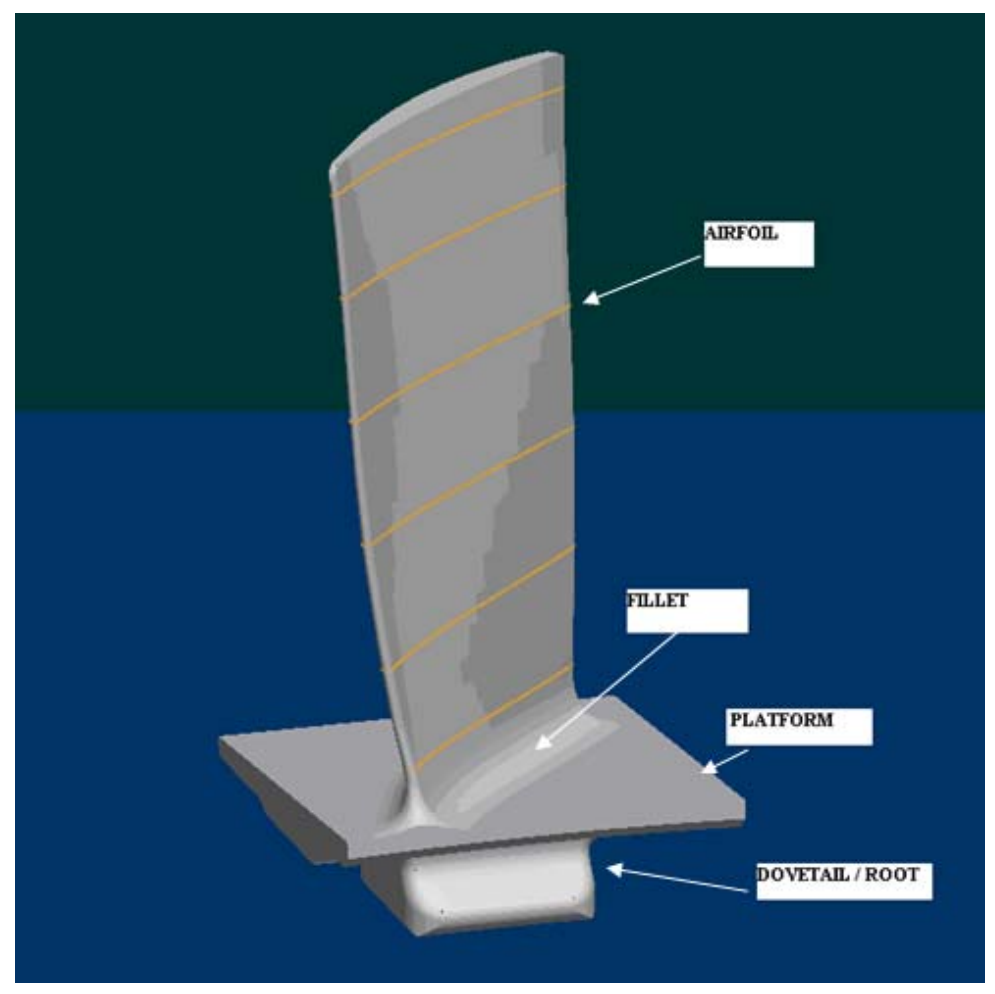

Figure 2-13: Typical Compressor Blade

\subsection{Aircraft Engines / Part families}

The airfoil design changes with different Original Equipment Manufacturer (OEMs), some of the most common engines out in the field in the commercial airline industry are the GE CF6-80 and CFM56 series engines. The CF6 series [15] is a family of high bypass turbo fan engines by General Electric. 
The major applications of the engine include Airbus A300, Airbus A330, Boeing 747, Boeing 767, and McDonnell Douglas DC-10. As shown in the Figure 2-14, the different stages of compressor blades that are being manufactured on this engine.
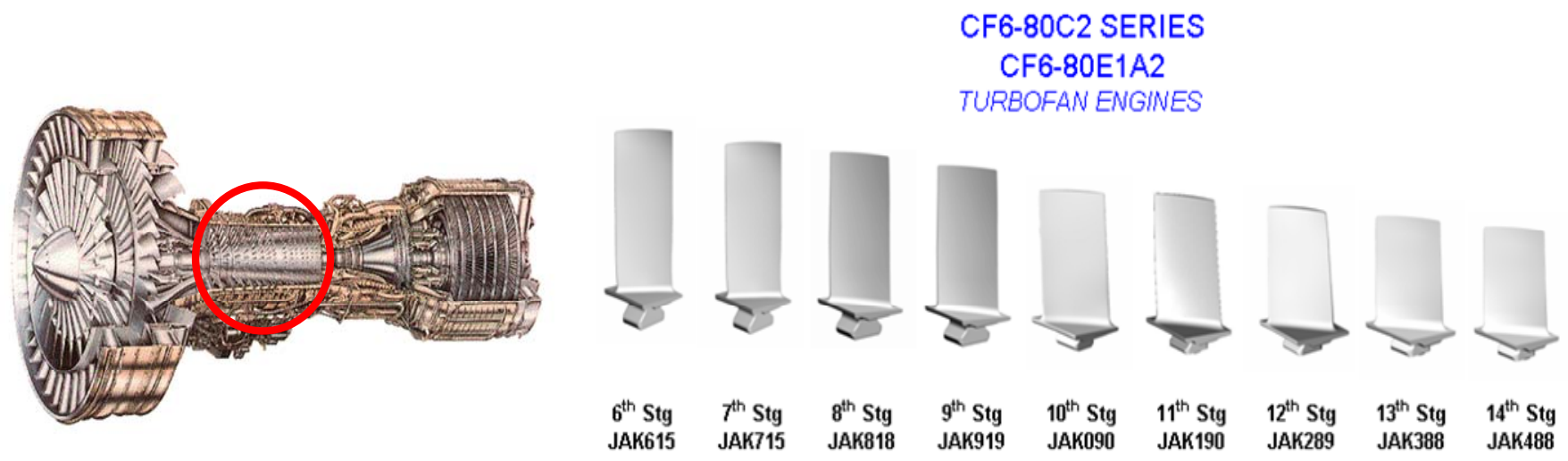

Figure 2-14: CF6 Engines Compressor Stages 6 to 14

CFM56 series is a family of high bypass turbofan engines made by joint venture between General Electric and SNECMA [16]. The major applications of the engine include Airbus [A320, A340], Boeing 737. As shown in Figure 2-15, the different stages of compressor blades that are being manufactured on this engine.
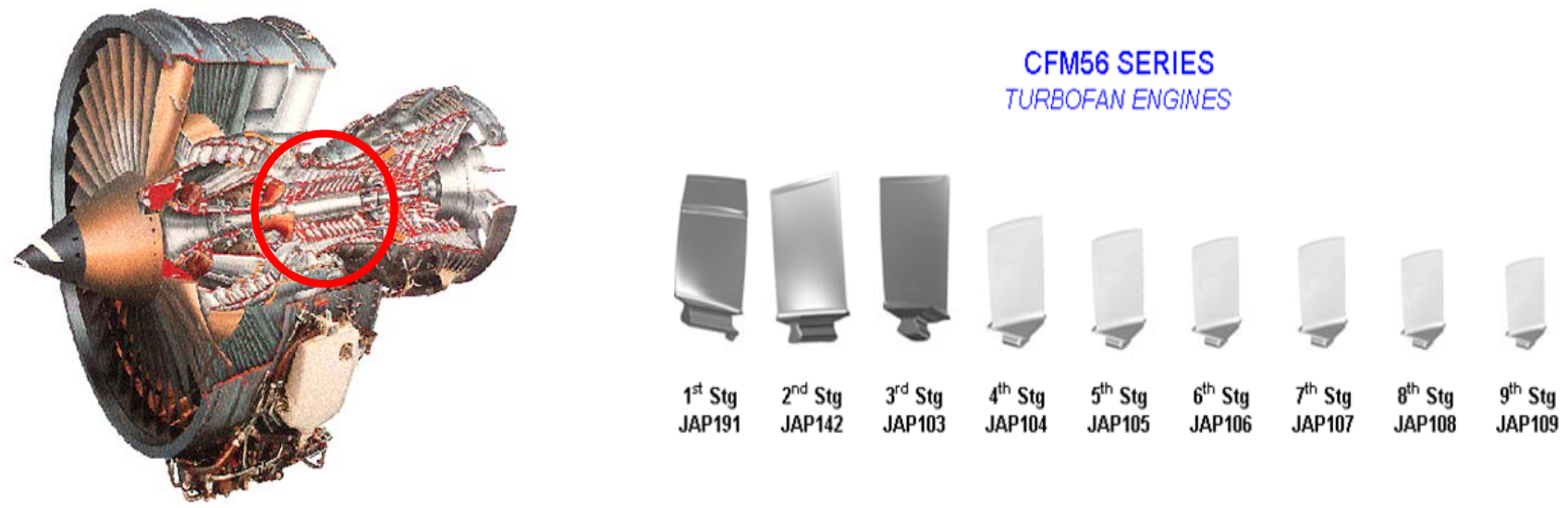

Figure 2-15: CFM56 Engines Compressor Stages 1 to 9 


\section{CHAPTER 3. COMPRESSOR BLADE MANUFACTURING PROCESS}

\subsection{Introduction}

Compressor blade manufacturing is a complex process where extra care is needed when handling the blades. Even tiny surface imperfections such as scratches, nicks and dings can lead to cracking of the blade when operating at full speeds. The impact of a cracked part can be detrimental to the performance of the engine and the aircraft itself. The manufacturing sequence is listed below by each operation for a better understanding of each process effect on blade features.

\subsection{Manufacturing Process}

The compressor blade manufacturing is divided into two main sections: the airfoil manufacturing and dovetail manufacturing. The airfoil manufacturing is subcontracted to a vendor who forges the airfoil to near net finish and ships the forgings to our business unit. Figure 3-1 shows a typical forging. These forgings are inspected using a CMM as soon as they are received and when found acceptable are released to the shop to have dovetail and further finish processing to manufacture a finished compressor blade. These forgings come in lots usually heat treated together and were assigned a heat code number to identify them as a batch for cases when the traceability is required to trace them back to the heat treatment operation at the vendor. To minimize variation within the lot the supplier is required to send all the parts together from the same heat code number.

The steps required to transform an incoming forging to a finished compressor blade are discussed below. 


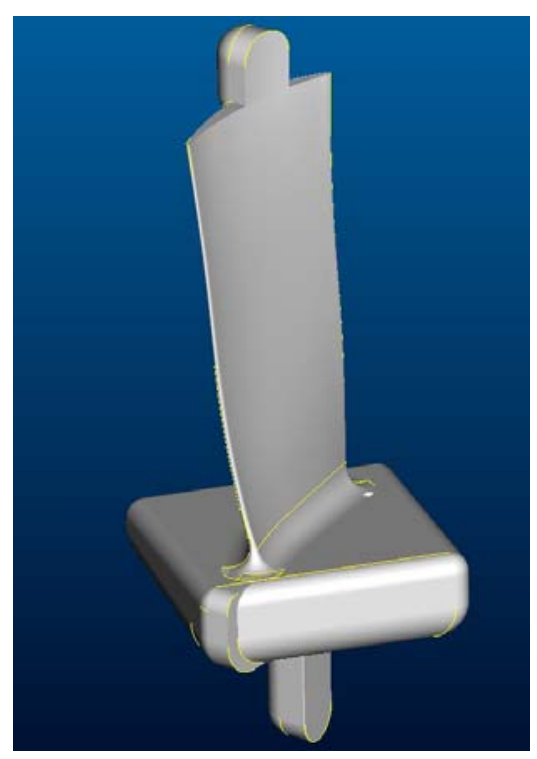

Figure 3-1: Typical forging with near net finish airfoil

\section{Encapsulation}

In general, encapsulation is the inclusion of one part within another substance so that the included part is not apparent. This process is extremely important and useful when an airfoil forging is surrounded by a material (usually a high-tech alloy) which is softer than the blade but strong enough to hold the blade in the fixtures, positioned in the desired direction and location. In essence it is holding the blade in a material to accommodate the processing of the blade which is otherwise impossible due to the complex shape of the blade. This makes the process of rough milling and root installation of a blade easier. The encapsulation material typically has a relatively low melting point so that the operator can melt and pour it around the blade [typically in a fixtures] to form the desired shape, but at the same time its melting point should be high enough to withstand the heat generated during roughing and root installation process. A common alloy that is used for the encapsulation process is CERROTRU [17]. A typical encapsulated blade is shown in the Figure 3-2. De-capsulation is the removal, or the making apparent, a part that was previously encapsulated. 


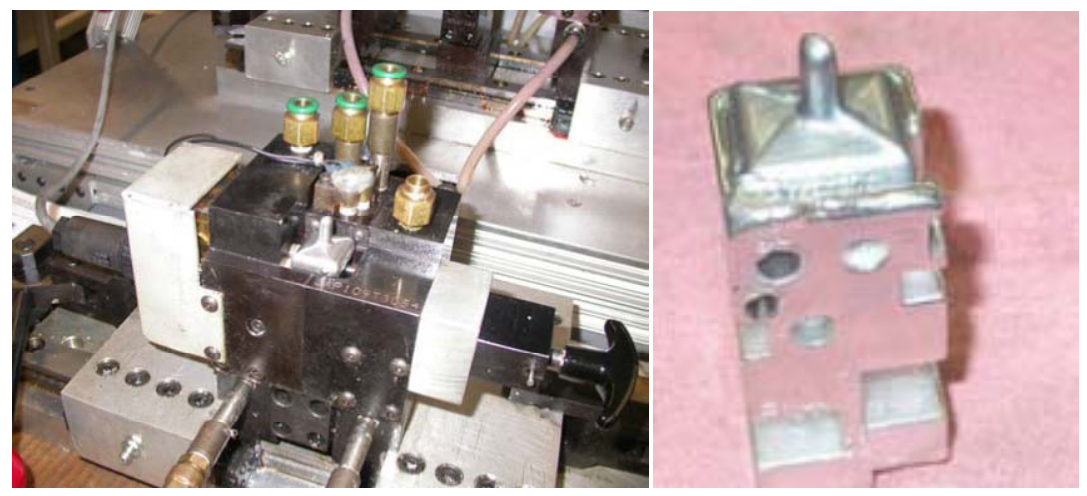

Figure 3-2: Encapsulation Fixture \& Encapsulated Part

\section{Rough Milling}

Rough Milling is a process in which the encapsulated part is milled to a desired shape and size to form a rough envelope for the next process to finish the remaining shape. This process is done essentially to reduce the stock that following process needs to work with, thereby saving the tool life and also reducing the processing time on the 5-axis grinder.

\section{Grinding}

Grinding is a process in which a machine tool is used for producing very fine finishes or make very light cuts, using an abrasive wheel as the cutting device. This wheel is made up of various sizes and types of stones, diamonds or of inorganic materials [18].

Typically, the grinding processes break down into three general categories. They are rough grinding, precision grinding and high or ultra precision grinding. The differentiating factor for each of these categories is the amount of metal removed. The metal removal is balanced against the desired tolerance or finish. In grinding, like turning and milling, high metal removal rates are generally in inversely proportional to close tolerances. This is main reason why manufacturers use roughing and finishing passes [18]. 
In rough grinding, the desired work piece/wheel interaction is focused on cutting. In these applications, maximum metal removal is the goal. Cutting off billets, snagging gates and risers from castings, or grinding weld beads smooth, are all processes where the maximum amount of metal removal is the goal. Precise control of the size and surface finish is a secondary consideration [18].

To create size and surface finish control for high metal removal in the precision grinding application, roughing passes are generally followed by finish passes. Precision grinding applications combine high metal removal with good part size control [18].

In ultra precision grinding operations, little or no actual cutting is done. Instead, the work piece surface is in effect rubbed clean primarily by sliding action from very fine abrasive grains. Ultra precision grinding is the surface finishing of a very precisely sized work piece. Most surface finishing processes generally fall into this category. These include lapping and polishing [18].

The grinding wheel designs are created using the finished part CAD models where the form of the dovetail is controlled extremely carefully. The 5-axis grinders install the entire dovetail features using the rough grinding wheel on the first few passes, and then finishing wheel cleans up for final finish.

The grinding process is where $80 \%$ of the airfoil/dovetail features are installed, leaving the remaining $20 \%$ for further finishing processes. A typical ground part is shown in the Figure 3-3, which shows an overlap of a forging (transparent green color) and a finished part (metallic color) to illustrate the transformation process. 


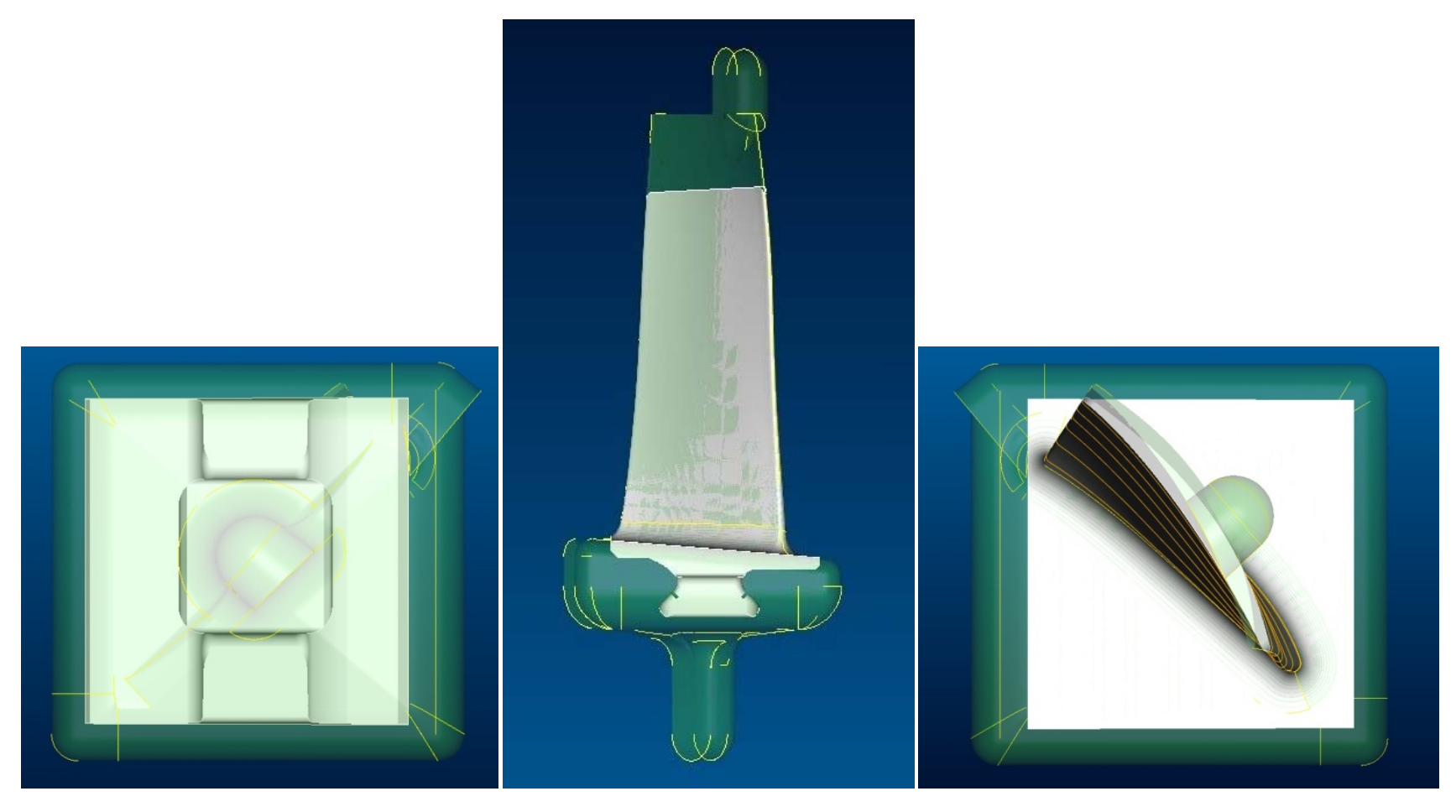

Figure 3-3: Forging (green) and Finished part (metallic) overlap view

\section{Polishing/Blending}

Polishing/Blending is the process by which the root features that were installed at the Root installation process are blended to obtain the desired uniform finish to achieve a smooth transition between the airfoil and dovetail. This process allows a smooth flow of the working fluid [compressed air] in the engine. In addition to that, it also cleans the burrs and raised material created by prior operations which could act as stress locators during the operational conditions resulting in the failure of the blade. This is an extremely important operation considering the impact of the finished blade on the engine performance. 


\section{ECG Tip Grinding}

The ECG process is used to cut the Tip of the airfoil to the desired length per design requirements, as shown in Figure 3-4. This process uses a combination of electrochemical and mechanical action to remove the material from the metals that are electrically conductive. There is a small gap between the wheel and the work piece due to the fact that the abrasive particles on the ECG wheel extend beyond the conductive bond surface. The electrolytic action begins when the gap is filled with an electrolyte, where the wheel acts are cathode and the work piece acts as the anode. Because of the electrochemical nature, the work piece is ground without significant contact to the metal; hence it produces pieces without burrs and without generating heat, distortion, or stress. Material removal occurs through a combination of electrochemical action which removes $90 \%$ of the material and mechanical grinding action, which removes the remaining $10 \%$ [19].

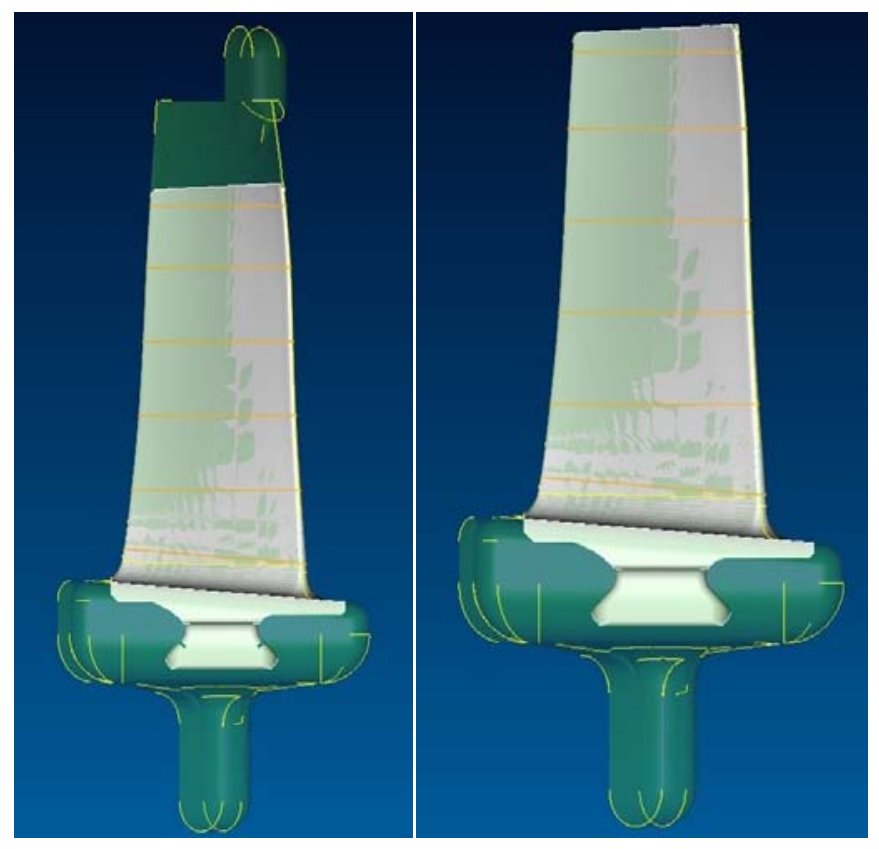

Figure 3-4: ECG tip ground part before and after 


\section{Pre-cleaning [ETCH]}

Etching is a process in which the surface of a material is altered by inducing a chemical reaction. This is a cleaning requirement to be carried out prior to FPI, which is discussed in the section below. The test surface should be free of any contamination s such as, oil, dirt, or grease that could keep the penetrant out of a defect such as cracks, dents etc. This can give false indications. Etching takes care of any kind of contamination which is why it is the most stable cleaning technique used in the aerospace industry. The etching process is also used to remove the top surface of the material depending on the concentration of the acid. In softer materials like titanium, the etch process is used to removed a portion of abusive machined layer.

\section{FPI- Fluorescent Liquid Penetrant Inspection}

FPI, or florescent penetrant inspection, is probably the most widely used NDT (non destructive testing) method used in the aerospace industry today. It entails pre-cleaning, which was discussed in the aforementioned section, the application of liquid florescent penetrant where the penetrant seeps into the defects (cracks) in the material after a dwell (wait) time, the careful removal of the liquid penetrant from the surface without removing it from the cracks, and finally a contrasting developer application which helps with easily reading the cracks against a black light as seen the Figure 3-5 below. A certified level I or II inspector usually does the readout of the compressor blades under the black light and dispositions the parts as conforming or non-conforming [20]. 

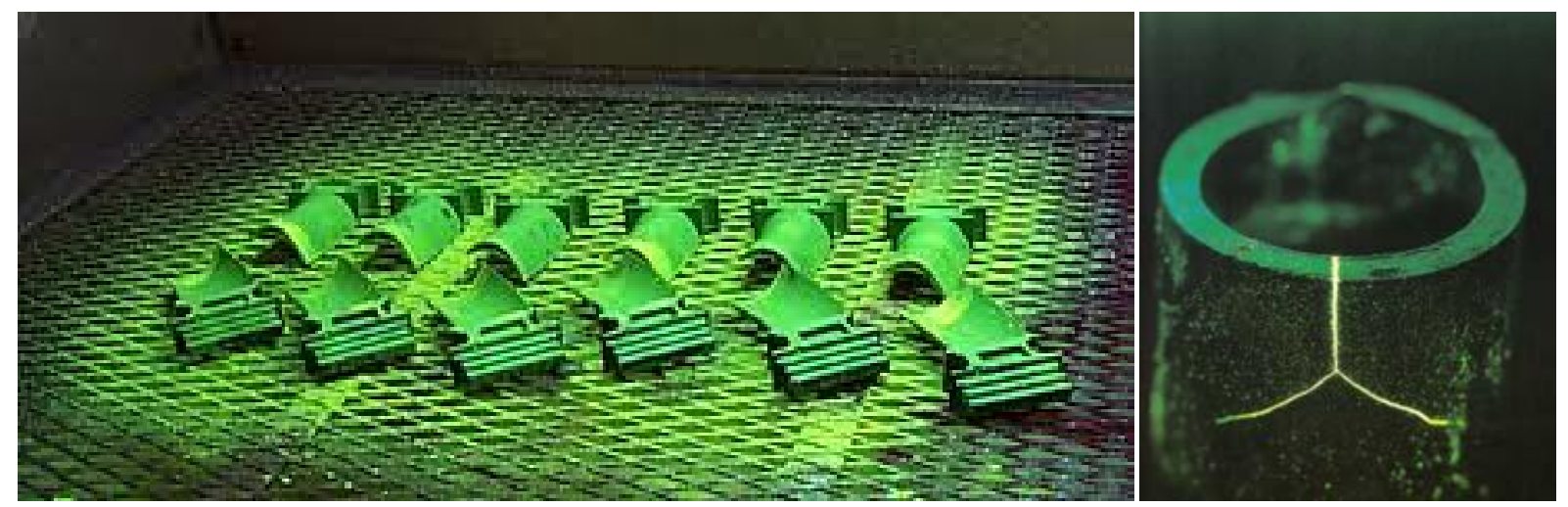

Figure 3-5: Penetrant application \& dwell, crack readout under a black light[21][22]

\section{Shot Peening}

After the compressor blades have passed through the FPI operation, they are moved on to shot peen. During shot peening, the airfoil undergoes a cold working process which is designed to introduce compressive stresses into the work piece in order to prevent propagation of surface cracks while the airfoils are operational. As the compressor blades move through the shot peen machine they are sprayed with cast steel shot at a designated intensity. As the shot contacts the surface of the part, it imparts small indentations, or dimples, to the surface of the blades, as shown in Figure 3-6. These dimples create a uniform compressive layer at the surface of the blades, which prevents all fatigue and stress corrosion failures. The shot peening process is also known to increase the fatigue strength of the part, which significantly increases the part life. The root of the blade is shot peened to a higher intensity than the airfoil [23]. 

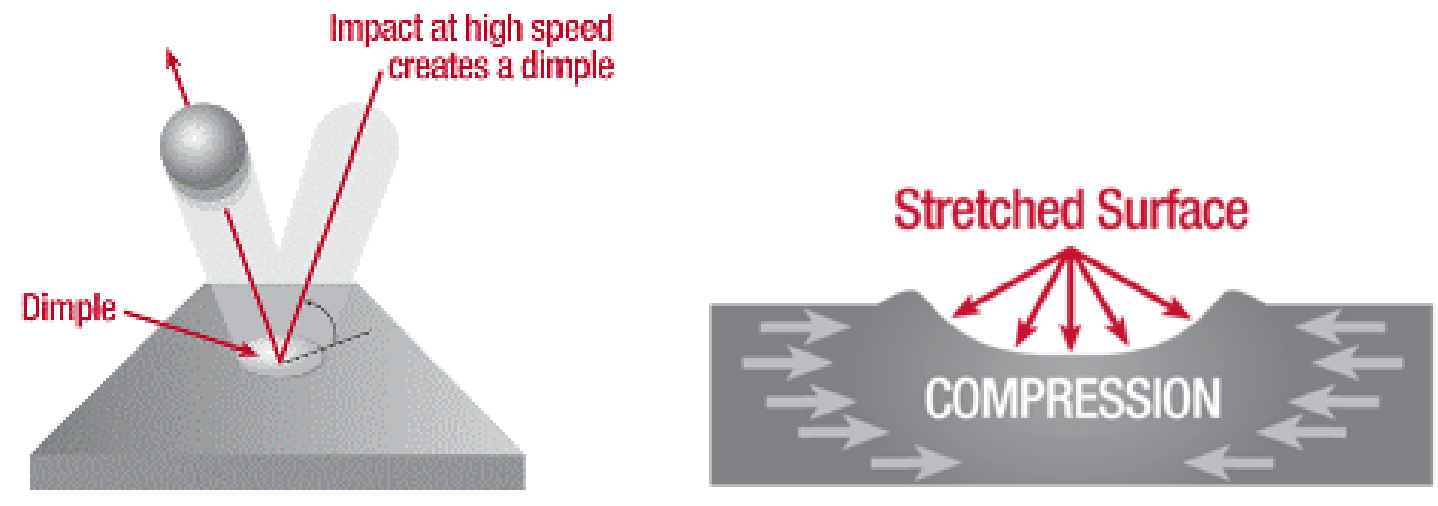

Figure 3-6: Shot peening dimple, compressive layer after shot peening

\section{Vibratory Mass Media Finish}

After the compressor blades have been through the shot peening operation, they go through a vibratory media finish operation. The vibratory media finish consists of cycling the compressor blades through selective media types of various sizes and shapes, as shown in Figure 3-7. The ceramic media rubs against the blades to carefully clean and polish the edges of the part and the overall blade. This operation uses the vibration of the tumbler to assist with the ribbing action along with a cleaning compound. The amplitude and vibration settings can be changed depending on the different size and shape of compressor blade stages.

This operation is essential to achieving the required surface finish per design requirements. It is ideal for finishing parts prior to painting, plating, heat treating, anodizing, and coating and sometimes it is the ideal final finish. As is the case with the compressor blades, they require a matte finish and vibratory media finish operation provides just that. 


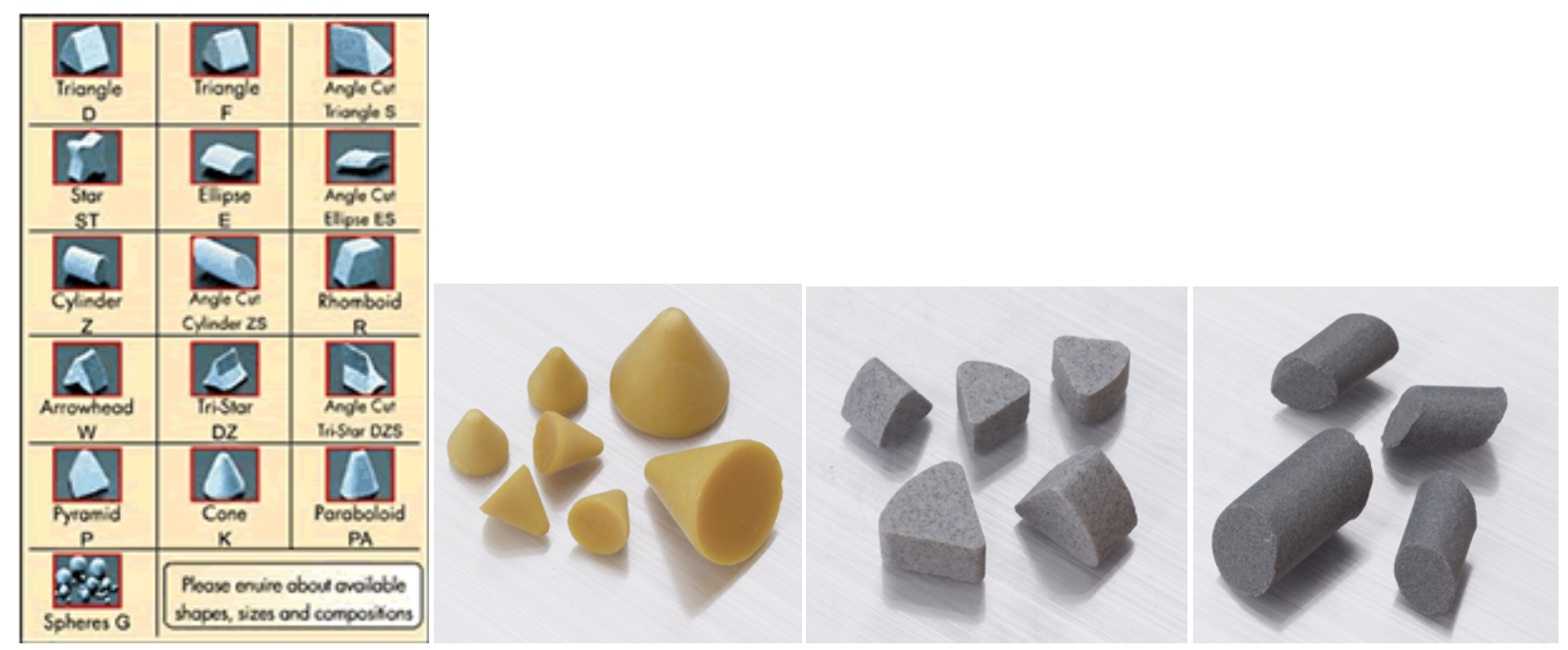

Figure 3-7: Different shapes \& sizes of media, ceramic \& plastic media

\section{Final Inspection}

Final inspection entails all the visual inspection, surface finish inspection, weight and other non dimensional requirements. After all the final inspection requirements are met, the parts are passed and packed and moved to stock to be shipped to the customer. 


\section{CHAPTER 4. COMPRESSOR BLADE INSPECTION}

\subsection{Introduction}

In looking back over the evolution of the measurement, since the days of ancient Egyptians building pyramids to modern day architecture, the measurement systems have come a long way to the point that measurement is an integral part of our everyday lives. Since the concept of interchangeable parts gained increased recognition, the automobile industry flourished with mass production, and as a result it was necessary to have parts made to absolute standards. The automation of machine tools created the need for faster and more flexible means of measuring. This requirement resulted in a new industry of threedimensional measuring machines. In recent times, the emphasis on Statistical Process Control (SPC) for quality improvement has accelerated the demand for faster and more accurate measurements. Coordinate Measuring Machines (CMM's) have become more capable to fulfill these growing requirements [24].

\subsection{Coordinate Measuring Machine}

A CMM is a great tool to reduce time taken to inspect complex parts. There are few limitations to the feature types whose dimensions cannot be measured by a CMM, as it depends on the size and shape of the part being inspected and as long as there is accessibility of the probe to the features, they can be measured. The flexibility coupled with accuracy of measurement is the reason why CMMs are widely accepted in the metrology world. One of the biggest advantages is the decreased inspection time which always translates into cost saving for the businesses [24].

The primary function of a CMM is to measure the actual shape of a workpiece, compare it against the desired shape, and evaluate the metrological information such as size, form, location, and orientation. 
The actual comparison is usually accomplished using data processing software with some advanced features to calculate complex feature dimensions [24].

The form of the workpiece is obtained by collecting a cloud of data points over the surface of the part. The data collection can be carried using contact and non-contact measuring heads. The data collection is carried using hard probing touch sensors that are scanning head and non-scanning head for continuous and discrete data points. Every measurement point is expressed in terms of its measured coordinates. Some sensors are capable of also collecting direction vectors of the measured points, which usually allows for better accuracies. However, it is not possible to evaluate the dimensional parameters directly from the measured coordinates. An analytical model is needed to compare it against the measured data to evaluate the parameters. The model contains ideal geometric data that is obtained usually from the CAD design. This is accomplished by applying the best-fit algorithms to fit the measured data set to the geometric model [24].

A standard CMM consists of following essential system components, as shown in Figure 4-1 [24]:

- A mechanical frame with three axes

- Probe head carrying the sensor that actually measures the part

- A control unit

- A computer with peripheral equipment (printer, plotter etc.) and software to calculate and display measurement results. The computer usually is connected to a network from where it can get programs and computer-aided design (CAD) files and it can send the measurement reports and data. 

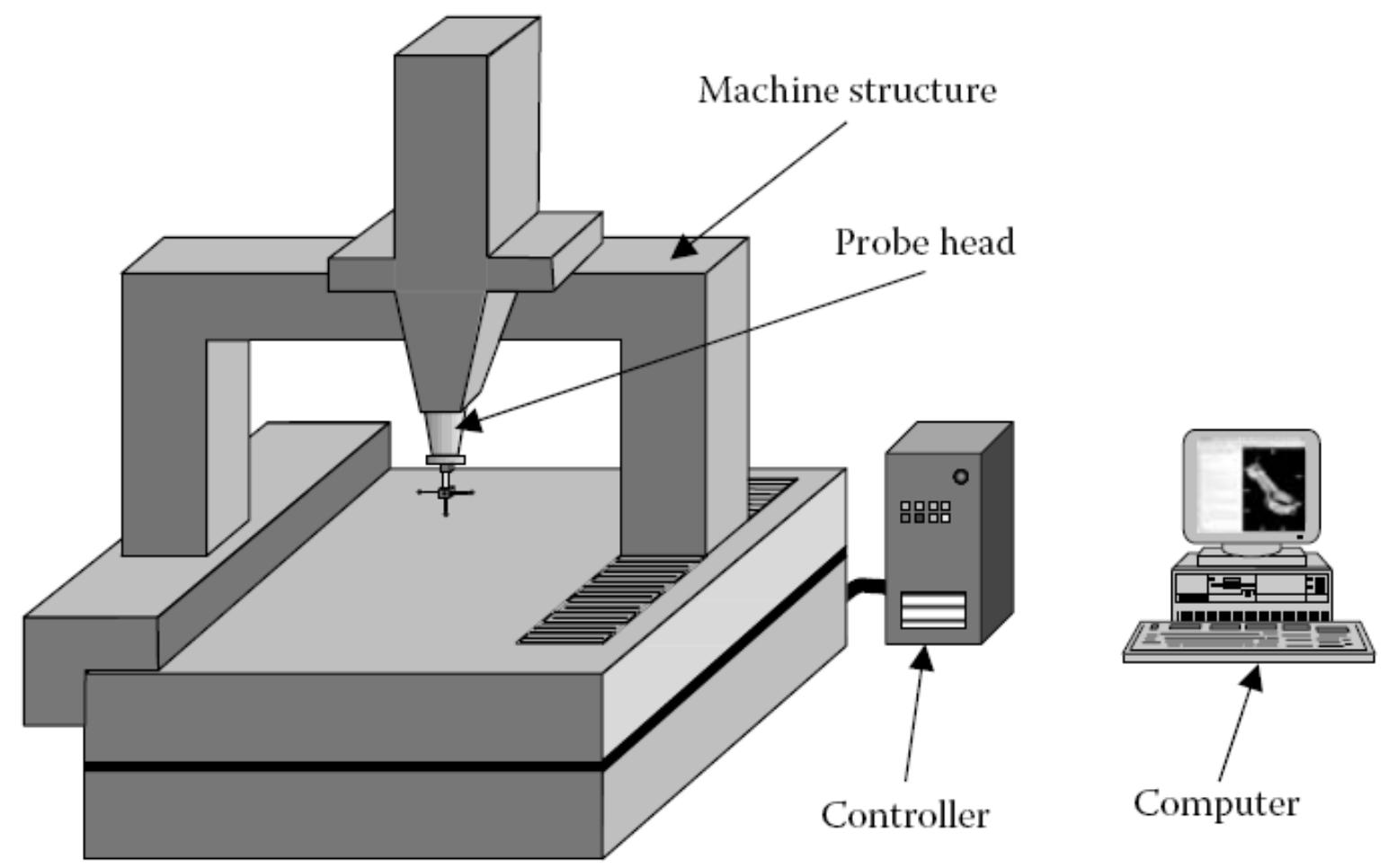

Figure 4-1: System Components of a CMM [24]

The three carriages of a CMM form a Cartesian reference coordinate system to which the probe head is attached. Transducers or scales determine the displacement along a coordinate path. This allows any point in the measurement volume of the CMM to be covered by the measurements using a spatial reference point on the probe head. This reference point is usually the center of the probe tip for contact sensors [24]. A measurement with a CMM comprises of the following steps:

- Calibration of the stylus or probe tip with respect to the probe head reference point, normally using a calibrated sphere (provided an electromechanical three-dimensional probe is used)

- Determination of the workpiece position and orientation (workpiece coordinate system) in relation to the machine coordinate system. 
- Measurement of the surface points on the workpiece

- Evaluation of the geometric parameters of the workpiece

- Representation or reporting of the measurement results

\subsection{Curve and Surface Fitting}

CMMs can measure a variety of features including sizes, forms, and locations for an extremely wide array of features simply provided that the CMM probe has the necessary access to the features. From its appearance, the CMM seems to only detect a collection of individual points. But it is, in fact, the software that processes these points that turns the CMM from a mere point collector into an immensely flexible, powerful measuring instrument [24].

A key component of CMM software is curve and surface fitting. Such fitting of CMM data points is necessary in order to assess feature size, location, or form deviation, or to establish a local coordinate system from datum features.

\subsection{Airfoil Data Processing (PC-DMIS Blade)}

PC-DMIS Blade software, developed by WILCOX Associates in partnership with various blade manufactures, is a turnkey solution for the analog scanning of blade sections. PC-DMIS Blade is a Visual Basic add-on to the basic PC-DMIS package. It has a simple to use interface, which lets you quickly identify parts, select the sections to measure and initiate scanning sequences [25].

PC-DMIS Blade uses traditional, section-based techniques to analyze blade measurements. Blade manufacturers have historically relied on guillotine gages to measure blade characteristics like contour and twist angles. These gages provide concise information, but they are expensive to make and 
maintain. A CMM using PC-DMIS Blade provides a faster, more flexible and less costly approach without compromising accuracy [25].

PC-DMIS Blade produces easy to understand graphical reports. Making blade measurement easy is only half of the equation. The second half is providing useful, concise information to operators on the shop floor. PC-DMIS Blade provides a wide range of outputs in simple to read, one-page reports. Users can configure it to report on important characteristics including things like chord width, leading edge thickness, twist angle, and mean camber line [25].

PC-DMIS Blade includes a range of alignment procedures. Proper alignment is the key to proper blade measurement. In addition to supporting the preferred method of root holding with XYZ offsets and Aangle rotation to the stacking axis, PC-DMIS Blade also supports 3D iterative alignments using either CAD surface models or 6 point rest [25].

\section{ASCII File}

The ASCII file contains airfoil section geometry definition that is defined by the drawing and the corresponding model, as shown in Figure 4-2. Section geometry is comprised of a series of point coordinates and corresponding normal vectors (as shown in Figure 4-3) derived from the parent airfoil surface. This data is used by the PCDMIS Blade software as the calculation basis for all airfoil section geometric characteristics defined in Chapter 2. 


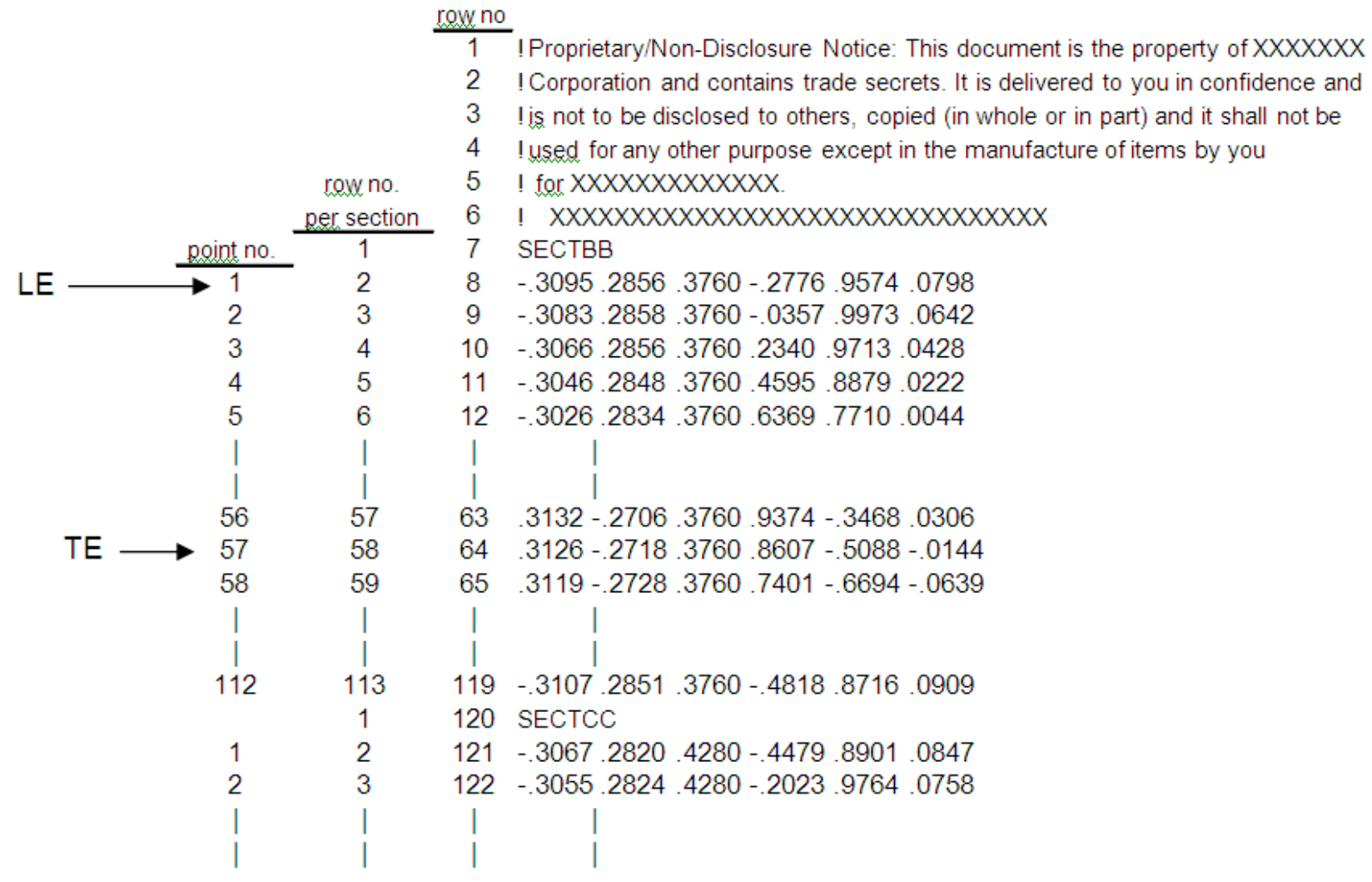

Figure 4-2: Sample ASCII file for a section of airfoil 


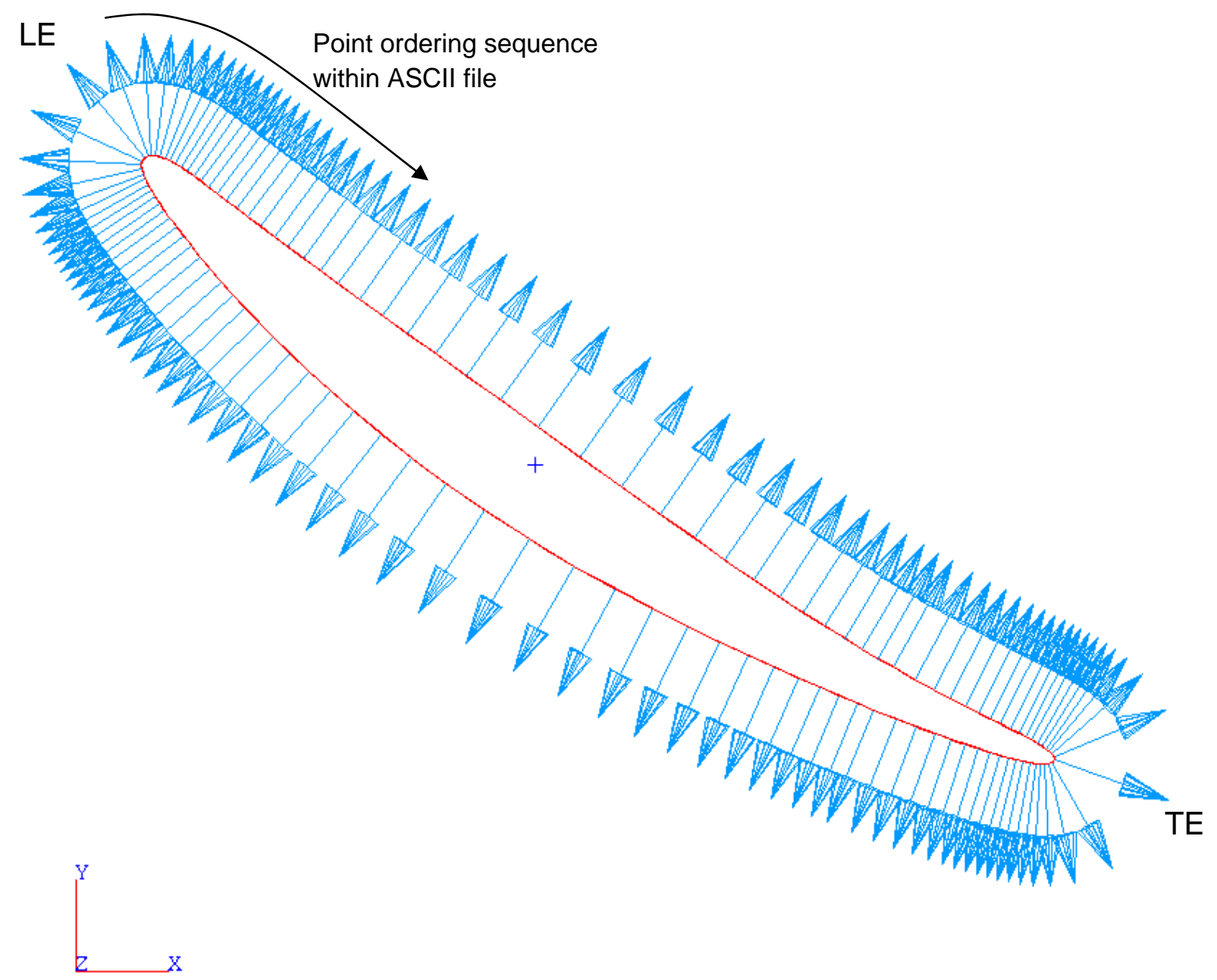

Figure 4-3: Airfoil Section Definition by Points and Local Normals 


\section{CHAPTER 5. SOFTWARE TOOL}

\subsection{Introduction}

The software tool was initially programmed in Minitab [26] using individual macros. Minitab is a powerful statistical analysis software when it comes to basic statistics, but it lacked the ability to program complex algorithms and mathematical equations. MATLAB, on the other hand, provided just the things Minitab was lacking, in addition to having the flexibility with data manipulation and visualization [27]. Once all the algorithms were tested, and validated in Minitab the program was rewritten in MATLAB for advanced programming flexibility.

\subsection{Processing Models}

Different stages of compressor blades were studied from forging to finish stage by inspecting all features using different heat code lots and the data was analyzed and compared to forging data to understand the processing effects. These processing effects were then formulated into each part-specific model that accurately estimated the airfoil feature tolerance variations from forging to finish process. The following section provides an overview of material types associated with the different stages of compressor blades. Due to proprietary reasons, process details and their effects are not discussed.

\subsection{Algorithms}

Each airfoil feature algorithms and its calculations that are packaged in the tool are discussed in this section. It describes the design and development of a software tool specific to each compressor blade feature that is being estimated. It is essential to have a thorough knowledge of compressor blade features discussed in Chapter 2 and compressor blade manufacturing process discussed in Chapter 3 to understand the material in this section. 


\section{True position of Centroid (XXX, YYY)}

These features are relatively straight forward to program. Since the root is installed after the airfoil has already being established, the operator has an enough room to install the root, of course within the allowed tolerance zone. Once established, these features have no significant changes in terms of shift from further processing of the blade except for shot peening. Shot peening with higher intensities outside the design tolerances has known to twist and bend the airfoil out of shape. Hence operating characteristics for the shot peening operations should be closely monitored and controlled to mitigate any risks of an operator error. The true position of the centroid is plotted using the tolerances obtained from the blue print for individual sections.

\section{Delta True Position (DTPXXX, DTPYYY, DTPN), Adjacent Section Deviation (ADJC, ADJMXT)}

The actual centroid locations of the above features must fall within their respective true position tolerance zones as shown in Figure 5-1. In addition, each adjacent centroid deviation must not exceed blueprint requirements. As the name implies, adjacent centroid deviation ('Delta True Position’ or DTP) is the calculated true position deviation difference between a given section and a section adjacent to it.

Table 5-1 and 5-2 show a calculation example of a compressor blade. Where $\mathrm{X}_{\mathrm{A}} \ldots \ldots \mathrm{X}_{\mathrm{E}}$ is the centroid deviation for their respective sections and “T” is Upper Specification Limit (USL) for that feature. Acceptance and rejection criteria are given by equation 1 and 2 respectively. Similar to true position, shot peening is the only process that has an effect on the DTP features.

$$
\begin{aligned}
& \text { If }\left|X_{I}-X_{J}\right|-T \leq 0 \text { then Accept } \\
& \text { If }\left|X_{I}-X_{J}\right|-T>0 \text { then Reject }
\end{aligned}
$$

Where $\mathrm{I}=\mathrm{B}, \mathrm{C}$....etc and $\mathrm{J}=\mathrm{A}, \mathrm{B}$...etc which is immediate adjacent section 
Table 5-1: Centroid deviation per section

\begin{tabular}{|c|c|}
\hline SECTION & $(\mathrm{XXX})$ Centroid deviation, in \\
\hline $\mathrm{AA}$ & $\mathrm{X}_{\mathrm{A}}$ \\
\hline $\mathrm{BB}$ & $\mathrm{X}_{\mathrm{B}}$ \\
\hline $\mathrm{CC}$ & $\mathrm{X}_{\mathrm{C}}$ \\
\hline $\mathrm{DD}$ & $\mathrm{X}_{\mathrm{D}}$ \\
\hline $\mathrm{EE}$ & $\mathrm{X}_{\mathrm{E}}$ \\
\hline
\end{tabular}

Table 5-2: Centroid deviation calculations

\begin{tabular}{|c|c|c|c|c|c|}
\hline Sect Pair & DTPXXX & ABS(DTPXXX) & USL & Difference & Disposition \\
\hline A-B & $\mathrm{X}_{\mathrm{B}-\mathrm{X}_{\mathrm{A}}}$ & $\mid \mathrm{X}_{\mathrm{B}-\mathrm{X}_{\mathrm{A}} \mid}$ & $\mathrm{T}$ & $\mid \mathrm{X}_{\mathrm{B}-\mathrm{X}_{\mathrm{A}} \mid-\mathrm{T}}$ & Accept/Reject \\
\hline B-C & $\mathrm{X}_{\mathrm{C}-\mathrm{X}_{\mathrm{B}}}$ & $\mid \mathrm{X}_{\mathrm{C}-\mathrm{X}_{\mathrm{B}} \mid}$ & $\mathrm{T}$ & $\mid \mathrm{X}_{\mathrm{C}-\mathrm{X}_{\mathrm{B}} \mid-\mathrm{T}}$ & Accept/Reject \\
\hline C-D & $\mathrm{X}_{\mathrm{D}-} \mathrm{X}_{\mathrm{C}}$ & $\left|\mathrm{X}_{\mathrm{D}-} \mathrm{X}_{\mathrm{C}}\right|$ & $\mathrm{T}$ & $\mid \mathrm{X}_{\mathrm{D}-\mathrm{X}_{\mathrm{C}} \mid-\mathrm{T}}$ & Accept/Reject \\
\hline D-E & $\mathrm{X}_{\mathrm{E}-\mathrm{X}_{\mathrm{D}}}$ & $\left|\mathrm{X}_{\mathrm{E}-} \mathrm{X}_{\mathrm{D}}\right|$ & $\mathrm{T}$ & $\left|\mathrm{X}_{\mathrm{E}-} \mathrm{X}_{\mathrm{D}}\right|-\mathrm{T}$ & Accept/Reject \\
\hline
\end{tabular}




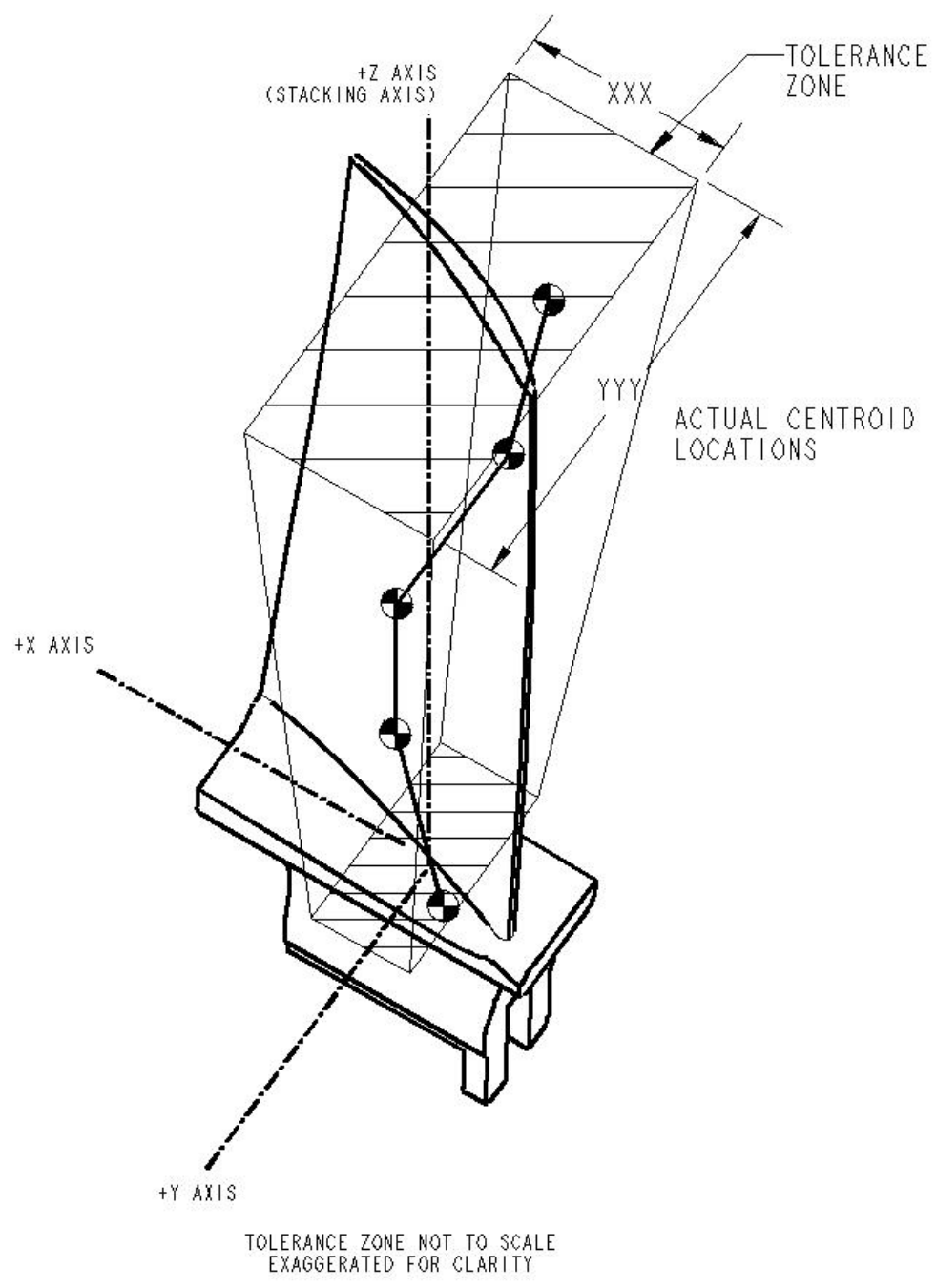

Figure 5-1: Airfoil Section Centroid Deviation Differences

\section{Chord Loss Simulation}

Chord changes from forging to final stages are mainly due to the Pre-FPI Etch process, Vibratory Media finish and shot peening processes. Etching is a process in which the surface of a material is altered by inducing a chemical reaction. As the material is removed, however small it might be, it has an effect on chord length. The same principle applies to Vibratory Media finish where the parts are moved through a non-abrasive media, where the media peens and pounds the edges and surface of the part. Depending on 
the length of time in the vibratory media finish the parts have shown to have some material loss. The shot peening process, on the other hand, entails impacting the surface of the blade with shot (cast steel, ceramic etc.) with force sufficient to create plastic deformation; this drastically alters the surface of the blade. Also, the fact that the blades are pre-twisted at the forging level and untwisted after the shot peening process has direct effect on the chord length.

Chord loss varies with the type of material for different compressor stage blades. Typical chord loss due to the above mentioned reasons ranges from .002 to .003 inches. But for softer alloys, like titanium, the chord loss is usually higher.

Various studies were conducted for different material types and different stages of the compressor blades using different heat codes chosen randomly. The methodology for conducting different studies and its results are out of the scope of this thesis. The chord loss function for a typical compressor blade is given by equation 3 :

$$
C_{F}=C+C_{L}
$$

Where

$C_{F}$ is the Chord Final;

$C$ is the Chord at forging level;

$C_{L}$ is the chord loss during the process.

Chord loss equations for nickel alloy, stainless steel and titanium alloy are given by equations 4, 5 and 6 respectively

$$
C_{L}=0.0-0.002 \times I D X / \operatorname{Max}(I D X)
$$




$$
\begin{aligned}
& C_{L}=-0.002-0.001 \times I D X / \operatorname{Max}(I D X) \\
& C_{L}=-0.002-0.002 \times I D X / \operatorname{Max}(I D X)
\end{aligned}
$$

Where $I D X$ is a sequential number allocated to each airfoil section from first to last; usually from $(0,1$, 2....etc.)

\section{Thickness Simulation (LET, TET, MXT)}

Similar to the Chord feature, the thickness features are affected by Pre-FPI etch process, vibratory media

finish and shot peening process. In fact, shot peening and vibratory media finish have a significant effect on the edge thickness as it the most exposed feature of the compressor blade. Thickness loss studies have been done to analyze various stages of the compressor blades using various heat codes. The thickness loss after final process is typically a constant value that is taken out of the forging thickness values. Final thickness loss is given by the equations 7, 8 and 9 for LET, TET and MXT respectively.

$$
L E T_{F}=L E T-T_{L}
$$

Where

$\operatorname{LET}_{F}$ is the final thickness

LET is the thickness at forging level for each section

$T_{L}$ is the thickness loss

$T E T$ and $M X T$ values are computed accordingly.

$$
\begin{aligned}
& T E T_{F}=T E T-T_{L} \\
& M X T_{F}=M X T-T_{L}
\end{aligned}
$$




\section{Profile Features (LEP, TEP, PSP, SSP, APP)}

The compressor blade profiles are critical features that affect the performance of the blade and the engine itself. These features also have an impact on the life of the blades; the efficiency of the fluid transfer between stages has a drastic effect on the efficiency of the engine. At first the all around profile deviation from the nominal is calculated after the least squares best-fit of the airfoil cross section. All other profile features are calculated after AAP is calculated. Please refer to Chapter 2 for airfoil geometry for further understanding these features.

All processing effects have an impact on the profile features, including Pre-FPI etch process, vibratory media finish and shot peening process. The profile tolerances won't change from forging to finish as the actual profile values are always best fitted to the nominal values.

\section{Peen Simulation (N-angle, LEA, and TEA)}

The shot peening operation is carried to produce a compressive residual stress layer and modify the mechanical properties of the metals. It entails impacting the surface with shot (cast Steel, glass, ceramic etc.) with force sufficient to create plastic deformation. Due to the high intensity of the shot peening, the airfoil tends to untwist after the shot peening process, and hence it is a common practice to introduce a pre-twist to compensate for the un-twist. These pre-twist values were studied across the different stages of compressor blades, and as with the other features, the amount of twist completely depends on the material of the compressor blade and also the intensity with which the surface being shot peened.

In order to provide the grind operator a simple way to target the N, LEA, and TEA with respect to the true position XXX and YYY, it is a common practice to center the data to the lowest section of the Nangle values. LEA and TEA are directly controlled by how the $\mathrm{N}$-angle is targeted, and they follow suit. 
Typically Section A is the most commonly used for $\mathrm{N}$-angle target, but it sometimes can be section B in cases where Section A is a reference section.

Several studies have been conducted to analyze the pre-peen and post-peen twist changes to N, LEA and TEA with respect to XXX and YYY. The methodology for conducting different studies and its results are out of the scope of this thesis. Please see the calculations below for a typical compressor blade; it usually ranges anywhere from 6 minutes on harder materials (Nickel Alloys) to 12 minutes on softer materials (Titanium Alloys). The peen simulation is given by equations 10, 11 and 12 for N-angle, LEA and TEA respectively.

$$
N_{F}=N_{I}+\text { OFFSET }+ \text { PEENCLOSURE }
$$

Where I = Sections (A, B ...etc)

$$
\begin{aligned}
& L E A_{F}=L E A_{I}+\text { OFFSET + PEENCLOSURE } \\
& T E A_{F}=T E A_{I}+\text { OFFSET + PEENCLOSURE }
\end{aligned}
$$

OFFSET $=$ Targeted Offset provided to the grind operator to maximize the yield of the lot, usually in set increments of +/-3 minutes [-21, -18, -15, -12, -9, -6, -3, 0, 3, 6, 9, 12, 15, 18, 21]

PEEN CLOSURE is the post peen un-twist for each specific Z-Prime at a set gage distance for each section.

Z-Prime vectors are calculated using the blueprint requirements of a Z-gage value taken at the stacking axis for each section label. Table 5-3 shows sample Z-prime vector calculations. 
Table 5-3: Z-prime vector calculations

\begin{tabular}{|c|c|c|}
\hline Section label & Z-gage distance at stacking axis & $\begin{array}{c}Z-P R I M E_{I}=\frac{\left(Z_{I}-Z_{A}\right)}{\left(Z_{G}-Z_{A}\right)} \\
\text { Where } \mathrm{I}=\mathrm{A}, \mathrm{B} \ldots \mathrm{G}\end{array}$ \\
\hline $\mathrm{A}$ & $\mathrm{Z}_{\mathrm{A}}=0.4$ & $\mathbf{. 0 0 0 0}$ \\
\hline $\mathrm{B}$ & $\mathrm{Z}_{\mathrm{B}}=0.55$ & $\mathbf{. 1 0 7 1}$ \\
\hline $\mathrm{C}$ & $\mathrm{Z}_{\mathrm{C}}=0.8$ & $\mathbf{. 2 8 5 7}$ \\
\hline $\mathrm{D}$ & $\mathrm{Z}_{\mathrm{D}}=1.05$ & $\mathbf{. 4 6 4 2}$ \\
\hline $\mathrm{E}$ & $\mathrm{Z}_{\mathrm{E}}=1.3$ & $\mathbf{. 6 4 2 8}$ \\
\hline $\mathrm{F}$ & $\mathrm{Z}_{\mathrm{F}}=1.55$ & $\mathbf{. 8 2 1 4}$ \\
\hline $\mathrm{G}$ & $\mathrm{Z}_{\mathrm{G}}=1.8$ & $\mathbf{1 . 0 0 0}$ \\
\hline
\end{tabular}

Peen Closure equations are different for each stage compressor blades and they vary based on the material type. Peen closure is given by equations 13, 14 and 15 for Nickel Alloys, stainless steel and titanium respectively. Typically peen closure of 6 minutes from root to tip is seen in Nickel Alloys, 12 minutes for stainless steel and 15 minutes for titanium alloys.

$$
\text { PEEN CLOSURE }=0.0-2.5 \times\left(Z-P R I M E_{I}\right)-3.72 \times\left(Z-P R I M E_{I}\right)^{2}
$$

Where I= A, B......G.

$$
\begin{aligned}
& \text { PEEN CLOSURE }=-1.0-2.36 \times\left(Z-P R I M E_{I}\right)-8.64 \times\left(Z-P R I M E_{I}\right)^{2} \\
& \text { PEEN CLOSURE }=0.0-11.465 \times\left(Z-P R I M E_{I}\right)-3.64 \times\left(Z-P R I M E_{I}\right)^{2}
\end{aligned}
$$




\section{Automatic N-Angle Targeting}

The ideal $\mathrm{N}$-angle offset should be calculated in a way that all three (N-angle, LEA and TEA) features for all sections for a given lot sample have the highest Cpk values, which essentially means that no part falls out of specification tolerances after final processing. Calculation of $\mathrm{N}$-angle offset can help the grind operator maximize the yield.

The algorithm that accomplishes the above is maximize999. The function of this algorithm is that, given the measured N-angle, LEA and TEA data, it returns an ideal N-angle that will provide the greatest postpeen yield. This is accomplished by maximizing both the lower centered data as well as the upper centered data as a function of N-Angle.

\section{Optimizing the N-Angle}

The theory behind finding the optimal N-Angle is that in order to maximize any yield using SPC (Statistical Process Control) is to have very small variations that are closely grouped around the nominal value, in other words, have close to zero deviation from the target value. This results in a high process capability $\left(\mathrm{C}_{\mathrm{pk}}\right)$ value. $\mathrm{C}_{\mathrm{pk}}$ is given by the Equation 16

$$
\mathrm{C}_{\mathrm{pk}}=\operatorname{Min}\left[\frac{(U C L-\bar{x})}{(3 \sigma)}, \frac{(\bar{x}-L C L)}{(3 \sigma)}\right]
$$

The function then generates post-peen $\mathrm{Cp}_{\mathrm{k}}$ data for both the upper and lower centered data for nonreference sections. That is, it generates both sets of data but does not assign either value as the $C p_{k}$ value for sections that are inspected. Instead, it compares the two values and finds the minimum difference between the two. Essentially, maximizer599 is finding the offset angle that will result in both the upper and lower centered data being as similar as possible and producing the greatest yield possible. 
Visually, as represented in the Figure 5-2 below, maximizing both the lower and upper centered data will result in an offset of approximately -12 minutes and an average Cpk of approximately 1.7.

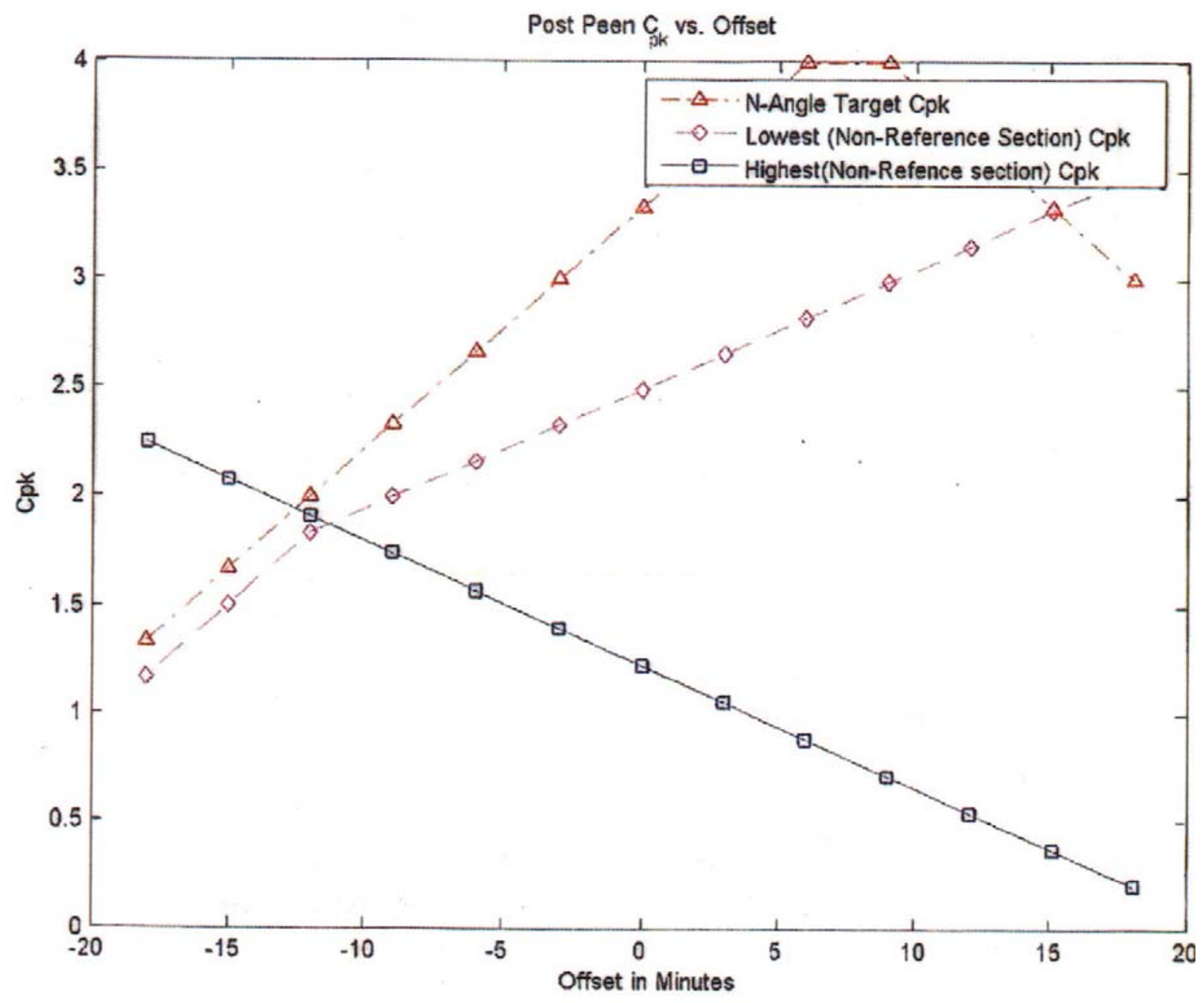

Figure 5-2: Visual representation fo post peen Cpk vs Offset

\subsection{Input data}

The forging lots that were received from the supplier have to be inspected using the CMM to accept or reject the lot. A random sample is taken from the lot for inspection; the sample size selection criteria used is based on MIL-STD-105E [28]. General inspection level II is used and based on single sample plan for normal inspection the sample size quantity of $10 \%$ (of the lot size ) or 20 minimum is used for 
selection. These parts are then inspected; the raw inspection data is processed through blade software that performs the liner and curvilinear fitting for each cross section based on the feature definitions. The data is then compared to the original reverse engineered airfoil section data comprising of a series of point coordinates and corresponding normal vectors to calculate the deviations for each feature. These deviations are then reported in a text file output which is used as an input to the software tool, developed during this project.

The input is then compiled in a spreadsheet which has airfoil, fillet and platform data each on a separate sheet in that order. Table 5-4 shows airfoil inspection data, Table 5-5 shows fillet inspection data and Table 5-6 shows platform inspection data.

Table 5-4: Airfoil Inspection Data

\begin{tabular}{|c|c|c|c|c|c|c|c|c|c|c|c|c|c|c|c|c|c|c|c|c|c|c|c|c|c|}
\hline & A & B & C & D & E & $\mathrm{F}$ & G & $\mathrm{H}$ & 1 & 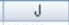 & K & L & M & N & 0 & $\mathrm{P}$ & Q & R & $s$ & $T$ & U & $V$ & W & $x$ & $Y$ \\
\hline 1 & pin & lot_code & insp & ib_ver & te_pt & units & SECT & $C N$ & $x X X+2$ & YYYH2 & C & N & CLEÅ & LEA & CTEA & TEA & LET & TET & MXT & A.PAma: & A.A.Pmin & LEP & PSP & TEP & SSP \\
\hline 2 & A2JAK289_D & A6.-246 & LPICMN & $10 \times 4.3$ & $\mathrm{MCL}$ & inłmin & A & 101 & $11-0.00387$ & 0.00174 & -0.00242 & -11.54 & $\begin{array}{l}4 \quad-5.97 \\
4\end{array}$ & $\quad-17.51$ & 12.94 & $\begin{array}{l}4 \quad 1.4 \\
\end{array}$ & $4 \cdot-0.00085$ & 0.00075 & -0.00139 & $\begin{array}{l}3 \\
3\end{array}$ & -0.00265 & 0.00171 & 0.00439 & 0.00231 & 0.00264 \\
\hline 3 & A2JAK289_D & A.6-246 & LPI_CMN & & $\mathrm{MCL}$ & inłmin & A & 102 & $2 \cdot-0.00329$ & 0.00072 & 0.00207 & -10.46 & 7.42 & -3.04 & 10.69 & 0.23 & -0.00007 & -0.00024 & -0.00059 & 0.0013 & -0.00149 & 0.00108 & 0.00279 & 0.00302 & 0.00091 \\
\hline 4 & A.JAKK289_D & A6-246 & LPICMN & $11 \times 4.3$ & $\mathrm{MCL}$ & inłmin & A & 103 & $\begin{array}{l}3-0.00336 \\
\end{array}$ & 0.00208 & 0.00214 & -6.79 & 6.47 & .0 .32 & 10.82 & 4.03 & $\begin{array}{c}3 \\
-0.00007\end{array}$ & -0.00034 & 0.00026 & 0.00144 & -0.00105 & 0.00096 & 0.0025 & 0.00281 & 0.00101 \\
\hline 5 & A2JAK289_D & A.6-246 & LPI_CMI & IN $v 4.3$ & $\mathrm{MCL}$ & inłmin & A & 104 & $\begin{array}{l}4 \cdot-0.00243 \\
\end{array}$ & 0.00085 & -0.00026 & -8.5 & 6.59 & -1.91 & 11.64 & 3.13 & 0.00019 & 0.0014 & 0.00136 & 0.00199 & -0.00068 & 0.00185 & 0.00267 & 0.00184 & 0.00109 \\
\hline 6 & A2JARK289_D & A.6-246 & LPI_CMI & Inv 4.3 & $\mathrm{MCL}$ & inłmin & A & 105 & $\begin{array}{ll}5 & -0.0025 \\
\end{array}$ & 0.00129 & 0.00165 & -5.18 & 6.55 & 1.37 & 10.41 & 5.23 & 0.00049 & -0.00096 & -0.00042 & 0.00103 & $\begin{array}{l}-0.00122 \\
\end{array}$ & 0.00176 & 0.00225 & 0.00363 & 0.00111 \\
\hline 7 & A2JAK289_D & A.6-246 & LPI_CMI & IN $v 4.3$ & $\mathrm{MCL}$ & indmin & A & 106 & $\begin{array}{cc}6 & -0.00324\end{array}$ & 0.00132 & 0.00294 & .12 .18 & 6.24 & .5 .94 & 13.05 & 0.87 & -0.00138 & -0.00044 & 0.00031 & 0.00133 & -0.00127 & 0.00215 & 0.0026 & 0.00332 & 0.00212 \\
\hline 8 & A2JAK289_D & $A 6-246$ & LPICCMI & 11044.3 & $\mathrm{MCL}$ & intmin & A & 107 & $7 \quad-0.00527$ & 0.00231 & -0.00195 & .15 .48 & 6.77 & -8.72 & 14.94 & -0.55 & -0.00018 & 0.00105 & -0.00235 & 0.00205 & -0.00253 & 0.00106 & 0.00458 & 0.0034 & 0.00203 \\
\hline 9 & A2JAK289_D & A.6-246 & LPICMN & IN $v 4.3$ & $\mathrm{MCL}$ & inłmin & A & 108 & -0.00183 & 0.00092 & -0.00399 & .5 .63 & .5 .99 & .11 .62 & 11.27 & 5.64 & 0.00063 & 0.00069 & 0.00021 & 0.00103 & -0.00117 & 0.0032 & 0.00208 & 0.00256 & 0.00182 \\
\hline 10 & A2JAK289_D & A.6-246 & LPICCMN & & ICL & intmin & A & 109 & $\begin{array}{ll}9 & -0.00246\end{array}$ & 0.00124 & 0.00142 & -6.79 & 6.09 & -0.7 & 11.1 & 4.31 & -0.00048 & -0.00033 & -0.00004 & 0.001 & -0.00132 & 0.00097 & 0.00232 & 0.0026 & 0.0013 \\
\hline 11 & A2JAK289_D & A.6-246 & LPICMN & 1104.3 & $\mathrm{MCL}$ & inłmin & & 110 & $\begin{array}{ll}0 & -0.00519 \\
\end{array}$ & 0.00304 & -0.00207 & -18.07 & 6.38 & -11.69 & 12.93 & -5.14 & -0.00119 & 0.00145 & -0.00075 & 0.00184 & -0.00204 & 0.0014 & 0.00388 & 0.00435 & 0.00161 \\
\hline 12 & A2JAK289_D & A.6-246 & LPI_CMI & IN 44.3 & & inłmin & A & 111 & 11.0 .0039 & 0.00272 & .0.00459 & -16.72 & 6.15 & -10.57 & 16.21 & .0 .51 & -0.00101 & .0 .00025 & -0.0012 & 0.0018 & -0.00289 & 0.00163 & 0.00469 & 0.00251 & 0.00272 \\
\hline 13 & A2JAK289-D & A.6-246 & LPI_CMI & & $\mathrm{MCL}$ & inłmin & A & 112 & -0.00116 & -0.00009 & 0.00247 & -6.49 & 6.42 & -0.08 & 12.19 & 5.7 & -0.00126 & -0.00014 & 0.00043 & 0.00132 & -0.00131 & 0.00124 & 0.00263 & 0.00242 & 0.00162 \\
\hline 14 & A2JAK289_D & A.6-246 & LPI_CMN & $1 \times v 4.3$ & $\mathrm{MCL}$ & intmin & A & 113 & -0.00184 & 0.00092 & 0.00039 & .7 & 7.71 & 0.71 & 11.11 & 4.12 & -0.00093 & 0.00042 & .0 .00082 & 0.00113 & -0.00142 & 0.00091 & 0.00256 & 0.00264 & 0.00123 \\
\hline 15 & A2JAK289_D & A.6-246 & LPICCMI & $11 \times 44.3$ & & inłmin & & 114 & $\cdot .0 .00422$ & 0.00282 & -0.00071 & -13.62 & 6.82 & -6.8 & 12.33 & -1.29 & 0.00005 & 0.00057 & -0.00042 & 0.00153 & -0.00142 & 0.00118 & & 0.00283 & 0.00084 \\
\hline 16 & A2JAK289_D & A6-246 & LPI_CMR & $1 \times \cup 4.3$ & $\mathrm{MCL}$ & intmin & A & 115 & .0 .00195 & 0.00084 & 0.00201 & -5.21 & 6.21 & 1 & 10.97 & 5.76 & $\cdot 0.00072$ & .0 .00011 & 0.00014 & 0.0015 & .0 .0009 & 0.00174 & 0.00237 & 0.00342 & 0.00137 \\
\hline 17 & A2JAK289_D & A.6-246 & LPI_CMN & IN $v 4.3$ & $\mathrm{MCL}$ & intmin & A & 116 & -0.00377 & 0.00255 & 0.00195 & .8 .23 & 7.88 & -0.35 & 11.18 & 2.94 & .0 .00121 & -0.00072 & .0 .00148 & 0.00096 & -0.00148 & 0.00183 & 0.00244 & 0.00354 & 0.00086 \\
\hline 18 & A2JAK289_D & A.6-246 & LPI_CMN & $10 \times 4.3$ & n & intmin & & 117 & .0 .00347 & 0.00161 & 0.00116 & -11.18 & 7.02 & .4 .17 & & 0.06 & .0 .00028 & 0.00065 & .0 .00023 & 0.00154 & -0.00147 & 0.00099 & & 0.00307 & 0.00127 \\
\hline 19 & A2JAK289_D & A.6-246 & LPI_CMN & In $v 4.3$ & M & intmin & A & 118 & $\begin{array}{r}8.0 .00289 \\
\end{array}$ & 0.00122 & 0.00417 & -10.22 & 6.41 & -3.81 & 10.86 & 0.64 & -0.00009 & -0.00036 & 0.0005 & 0.00127 & -0.0008 & 0.00127 & 0.00208 & 0.00244 & 0.00137 \\
\hline 20 & A2JAK 289 & A.6-246 & LPICCMN & $11 \times 4.3$ & $\mathrm{MCL}$ & inłmin & A & 119 & $\begin{array}{cc}-0.00436 \\
\end{array}$ & 0.00206 & 0.00036 & -9.27 & .5 .94 & -15.21 & 12.82 & 3.55 & 0.00136 & -0.00025 & -0.00124 & 0.00164 & -0.00237 & 0.00109 & 0.00401 & 0.00195 & 0.0014 \\
\hline 21 & A2JAK289_D & A.6-246 & LPI_CMN & $11 \times 4.3$ & M & intmin & & 120 & -0.0026 & 0.00137 & 0.00206 & .7 .37 & 6.9 & -0.47 & 10.94 & 3.58 & 0.00063 & 0.00047 & 0.00074 & 0.00138 & -0.00128 & 0.00208 & 0.00266 & 0.00236 & 0.00132 \\
\hline 22 & A2JAK289_D & A6-246 & LPI_CMR & & & intr & & 101 & & 0.00273 & -0.00138 & -13.4 & & -6.4 & & 2.2 & 0.00 & 0.00062 & -0.0013 & 0.00186 & -0.0 & 0.0011 & 0.00416 & 0.00149 & 0.00132 \\
\hline 23 & A2JAKK289_D & $A 6-246$ & LPI_CMI & $10 \times 4.3$ & $\mathrm{MCL}$ & intmin & & 102 & -0.0034 & 0.00204 & 0.00161 & .9 .98 & 10.76 & 0.78 & 8.13 & .1 .85 & 0.00103 & .0 .00018 & -0.00114 & 0.00102 & . 0.00124 & 0.00099 & 0.00226 & 0.00232 & 0.00176 \\
\hline 24 & A2JAK289_D & A.6-246 & LPI_CMN & IN $\cup 4.3$ & & intmin & & 103 & -0.00349 & 0.00254 & 0.00186 & .5 .34 & 6.27 & 0. & & 4.18 & & .0 .00124 & -0.00149 & 0.00094 & .0 .00107 & 0.00191 & 0.00191 & 0.00315 & 0.00185 \\
\hline 25 & A2JAK289_D & A.6-246 & LPI_CMI & IN $v 4.3$ & & & & 104 & $\begin{array}{r}-0.00297 \\
4\end{array}$ & 0.00174 & .0 .0002 & & & .0 & & 0.44 & & & 0.00052 & 0.00 & & & & 0.0017 & 0.00123 \\
\hline 26 & A2JAK289_D & A.6-246 & LPI_CMN & IN $v 4.3$ & & intmi & & 105 & $\begin{array}{ll}5 & -0.0031\end{array}$ & 0.00268 & 0.00121 & .4 & 7.8 & 3.5 & 5.04 & 0.7 & 0.00119 & -0.00173 & -0.0011 & 0.00066 & -0.00143 & 0.00264 & 0.00128 & 0.00382 & 0.00209 \\
\hline 27 & A2JAK 289-D & A6-246 & LPI_CMI & $11 \times 44.3$ & & intm & & 106 & -0.00332 & 0.0025 & 0.00309 & .12 .24 & 8.39 & -3.85 & 15.1 & 2.86 & -0.0 & -0.00084 & -0.00043 & 0.00134 & -0.0 & 0.00118 & 0.00267 & 0.00315 & 0.00168 \\
\hline 28 & A2JAK289-D & A.6-246 & LPI_CMN & IN $\cup 4.3$ & & & & 107 & & & -0.00216 & -10.07 & 10.8 & 0.7 & 10.84 & 77 & & 0.0007 & & 0.00 & & 0.0017 & & 0.00343 & 0.00162 \\
\hline 29 & JAK289_D & A6-246 & LPI_CMI & IN 44.3 & & & & 108 & -0.0 & & -0.00174 & -3.16 & 4. & & & 1.67 & 0.00 & -0.001 & -0.00 & 0.00007 & -0.00125 & 0.00278 & 084 & 0.00348 & 0.00132 \\
\hline 30 & JAK289_D & A.6-246 & LPI_CMN & IN 44.3 & & & & 109 & & 255 & 0.00338 & .9 & 7.1 & -1. & & 48 & -0.00026 & -0.0018 & .0 .00 & 0.00 & -0.0013 & 0.00104 & 0.00 & 0.00338 & 0.00161 \\
\hline 31 & JAK289_D & A.6-246 & LPICCMI & & & & & 110 & -0.00 & 0.0 & 0.00 & -12.94 & 6.23 & -6 & & .81 & & & & & & & & & \\
\hline 32 & $\mathrm{~A}_{2}$ & $86-246$ & LPI_CMI & IN $v 4.3$ & & & & 111 & $1 \cdot .0 .00$ & 0.00355 & .0 .00296 & -13.1 & & .7 .0 & & 6.19 & $\begin{array}{ll}9 & -0.00 \\
\end{array}$ & .0 .00 & & 0.00 & -0.0 & 0.00061 & & 0.00351 & 0.00186 \\
\hline 33 & A2JAK289_D & A.6-246 & LPI_CMR & $14 \times 4.3$ & & intr & & 112 & & 0.0018 & 0.0023 & .8 .72 & & 1. & & 0.72 & $2 \quad 0.0002$ & -0.00116 & -0.00 & 0.00 & -0.00 & 0.00066 & 0.00 & 0.00309 & 0.00159 \\
\hline 34 & A2JAK 289 & A.6-246 & LPICMN & $10 \times 4.3$ & & & & 113 & $\begin{array}{ll}3 & -0.00 \\
\end{array}$ & & 0.00 & -9.4 & & & & -2.77 & -0.0 & -0.00058 & & 0.00 & & 0.00 & 0.00 & 0.00247 & 0.0015 \\
\hline 35 & JAK289_D & A6-246 & LPI_CMI & IN $\cup 4.3$ & & & & 114 & $\begin{array}{l}4 \\
+\quad-0.0\end{array}$ & 0.0 & 0.0 & -10.05 & 9 & .0 .3 & 10.24 & 0.19 & 0.00048 & -0.00054 & -0.00 & 0.00131 & $\begin{array}{l}-0.00087 \\
\end{array}$ & 0.00151 & 0.00219 & 0.00349 & 0.00113 \\
\hline 36 & 9 & A6-246 & LPICCMN & $10 \times 4$ & & & & & & 0.0 & & & & & & & -0.00 & -0.00097 & -0.00 & 0.0006 & & 0.00125 & 0.00164 & 0.00304 & 0.00138 \\
\hline 37 & $\mathrm{~A}_{2}$ & $8.6-246$ & LPL_CM & & & & & 116 & & & & .8 & & & & & -0.0 & & -0.0 & 0.0 & & 0.0 & & 0.00347 & 0.00156 \\
\hline 38 & A2JAK 289_D & A6-246 & LPI_CMI & IN 44.3 & & & E & 117 & -0.00 & & & & 7.03 & -1.1 & 9. & 1.21 & 0.00091 & -0.0004 & -0.0003 & 0.0009 & -0.00105 & 0.00177 & 0.00195 & 0.00284 & 0.00138 \\
\hline 39 & A2JAK289_D & $8,6-246$ & LPI_CMI & Iv $\sim 4.3$ & $\mathrm{MC}$ & intrin & B & 118 & .0 .0 & 0.0026 & 0.00322 & .10 .53 & 9.2 & -1.33 & 10.75 & 0.22 & -0.00003 & .0 .00078 & -0.00051 & 0.00098 & -0.00093 & 0.00068 & 0.00191 & 0.00258 & 0.00157 \\
\hline 40 & A2JAK289_D & A.6-246 & LPI_CMI & & & & $\mathrm{B}$ & 119 & & & .0 .00099 & & & & 12.2 & & & -0.00044 & -0.00148 & 0.00128 & -0.00217 & & 0.00345 & & 0.00101 \\
\hline 41 & A2JAK 289 & A6-246 & LPICCMN & $10 \times 4.3$ & & & B & 120 & -0.00 & & & & & 0.4 & & 2.22 & & -0.00116 & & 0.00 & -0.0 & 0.00102 & 0.00131 & 0.00276 & 0.00186 \\
\hline 42 & A2JAKK289_D & $86-246$ & LPI_CMI & IN $v 4.3$ & $\mathrm{MCL}$ & intm & c & 101 & .0 .00388 & 0.00402 & 0.00008 & .7 .78 & 4.8 & .2 .93 & 12.92 & 5.14 & 0.00067 & 0.00136 & -0.00083 & 0.00175 & -0.00127 & 0.00111 & 0.00302 & 0.00127 & 0.00088 \\
\hline 43 & A2JAK 289 & A.6-246 & LPI_CM & & & & o & & & & & & & & & & & & & & & & & & \\
\hline 44 & A2JAK2 & $8,6-2$ & LPI_CMI & $10 \times 4.3$ & & & c & 1 & & & & 1.2 & & & & 11.32 & & & & & & 163 & & 0.00164 & 0.00124 \\
\hline 45 & A2JAKK289-D & A6-246 & LPICCMI & $11 \times v 4.3$ & $\mathrm{MCL}$ & indmin & C & 104 & -0.00371 & 0.00332 & 0.00115 & -2.65 & 4.62 & 1.97 & 9.75 & 7.1 & 0.0012 & 0.00054 & 0.00004 & 0.00136 & -0.00054 & 0.00174 & 0.00191 & 0.00115 & 0.00097 \\
\hline 46 & A2JAK289_D & A6-246 & LFI_CMR & $11 \times 44.3$ & $\mathrm{MCL}$ & indmin & c & 105 & -0.00349 & 0.0041 & 0.00036 & 1.69 & 5.95 & 7.63 & 7.97 & 9.65 & 0.00087 & -0.00113 & -0.00046 & 0.00055 & -0.00101 & 0.00178 & 0.00126 & 0.00219 & 0.00146 \\
\hline & & & & & & & & & & & & & & & & & & & & & & & & & \\
\hline
\end{tabular}


Table 5-5: Fillet Inspection Data

\begin{tabular}{|c|c|c|c|c|c|c|c|}
\hline 4 & A & $B$ & C & D & $E$ & $\mathrm{~F}$ & G \\
\hline 1 & 101 & DCV3 & $\mathrm{M}$ & 0.0025 & 0.0025 & 0.006 & 0 \\
\hline 2 & 102 & DCV 3 & M & 0.0024 & 0.0024 & 0.006 & 0 \\
\hline 3 & 103 & DCV3 & $\mathrm{M}$ & 0.0024 & 0.0024 & 0.006 & 0 \\
\hline 4 & 104 & DCV3 & $\mathrm{M}$ & 0.0021 & 0.0021 & 0.006 & 0 \\
\hline 5 & 105 & DCV3 & $\mathrm{M}$ & 0.0024 & 0.0024 & 0.006 & 0 \\
\hline 6 & 106 & DCV 3 & $\mathrm{M}$ & 0.0026 & 0.0026 & 0.006 & 0 \\
\hline 7 & 107 & DCV 3 & $\mathrm{M}$ & 0.0019 & 0.0019 & 0.006 & 0 \\
\hline 8 & 108 & DCV 3 & $\mathrm{M}$ & 0.002 & 0.002 & 0.006 & 0 \\
\hline 9 & 109 & DCV 3 & $\mathrm{M}$ & 0.003 & 0.003 & 0.006 & 0 \\
\hline 10 & 110 & DCV 3 & M & 0.0025 & 0.0025 & 0,006 & 0 \\
\hline 11 & 111 & DCV 3 & $\mathrm{M}$ & 0.0039 & 0.0039 & 0.006 & 0 \\
\hline 12 & 112 & DCV3 & $\mathrm{M}$ & 0.0018 & 0.0018 & 0.006 & 0 \\
\hline 13 & 113 & DCV3 & $\mathrm{M}$ & 0.0027 & 0.0027 & 0.006 & 0 \\
\hline 14 & 114 & DCV3 & $\mathrm{M}$ & 0.0009 & 0.0009 & 0.006 & 0 \\
\hline 15 & 115 & DCV3 & $\mathrm{M}$ & 0.0034 & 0.0034 & 0.006 & 0 \\
\hline 16 & 116 & DCV 3 & $\mathrm{M}$ & 0.0028 & 0.0028 & 0.006 & 0 \\
\hline 17 & 117 & DCV3 & $\mathrm{M}$ & 0.002 & 0.002 & 0.006 & 0 \\
\hline 18 & 118 & DCV 3 & $\mathrm{M}$ & 0.0029 & 0.0029 & 0.006 & 0 \\
\hline 19 & 119 & DCV 3 & M & 0.002 & 0.002 & 0.006 & 0 \\
\hline 20 & 120 & DCV 3 & $\mathrm{M}$ & 0.0018 & 0.0018 & 0.006 & 0 \\
\hline 21 & 101 & DCV4 & $\mathrm{M}$ & 0.0035 & 0.0035 & 0.006 & 0 \\
\hline 22 & 102 & DCV4 & $\mathrm{M}$ & 0.0026 & 0.0026 & 0.006 & 0 \\
\hline 23 & 103 & DCV4 & $\mathrm{M}$ & 0.0028 & 0.0028 & 0.006 & 0 \\
\hline 24 & 104 & DCV4 & $\mathrm{M}$ & 0.0021 & 0.0021 & 0.006 & 0 \\
\hline 25 & 105 & DCV4 & $\mathrm{M}$ & 0.0025 & 0.0025 & 0.006 & 0 \\
\hline 26 & 106 & DCV4 & $\mathrm{M}$ & 0.0031 & 0.0031 & 0.006 & 0 \\
\hline 27 & 107 & DCV4 & $\mathrm{M}$ & 0.0022 & 0.0022 & 0.006 & 0 \\
\hline 28 & 108 & DCV4 & $\mathrm{M}$ & 0.0021 & 0.0021 & 0.006 & 0 \\
\hline 29 & 109 & DCV4 & $\mathrm{M}$ & 0.0034 & 0.0034 & 0.006 & 0 \\
\hline 30 & 110 & DCV4 & $\mathrm{M}$ & 0.0029 & 0.0029 & 0.006 & 0 \\
\hline 31 & 111 & DCV4 & $\mathrm{M}$ & 0.0047 & 0.0047 & 0.006 & 0 \\
\hline 32 & 112 & DCV4 & $\mathrm{M}$ & 0.0021 & 0.0021 & 0.006 & 0 \\
\hline$\frac{12}{14}$ & $\begin{array}{l}112 \\
\text { Sheet }\end{array}$ & ONys & on & Canas & n $10 \mathrm{nay}$ & a nor & \\
\hline
\end{tabular}

Table 5-6: Platform Inspection Data

\begin{tabular}{|c|c|c|c|c|c|c|}
\hline 4 & A & C & D & $E$ & $\mathrm{~F}$ & G \\
\hline 1 & 101 IC5 & Y & -0.0764 & 0 & 0.0002 & 0.0002 \\
\hline 2 & 102 IC5 & Y & -0.0764 & 0 & 0.0002 & 0.0002 \\
\hline 3 & 103 IC5 & Y & -0.0764 & 0 & 0.0002 & 0.0002 \\
\hline 4 & 104 IC5 & Y & -0.0764 & 0 & 0.0002 & 0.0002 \\
\hline 5 & 105 IC5 & Y & -0.0764 & 0 & 0.0002 & 0.0002 \\
\hline 6 & 106 IC5 & Y & -0.0764 & 0 & 0.0002 & 0.0002 \\
\hline 7 & 107 IC5 & Y & -0.0764 & 0 & 0.0002 & 0.0002 \\
\hline 8 & 108 IC5 & Y & -0.0764 & 0 & 0.0002 & 0.0002 \\
\hline 9 & 109 IC5 & Y & -0.0764 & 0 & 0.0002 & 0.0002 \\
\hline 10 & 110 IC5 & Y & -0.0764 & 0 & 0.0002 & 0.0002 \\
\hline 11 & 111 IC5 & Y & -0.0764 & 0 & 0.0002 & 0.0002 \\
\hline 12 & 112 IC5 & Y & -0.0764 & 0 & 0.0002 & 0.0002 \\
\hline 13 & 113 IC5 & Y & -0.0764 & 0 & 0.0002 & 0.0002 \\
\hline 14 & 114 IC5 & Y & -0.0764 & 0 & 0.0002 & 0.0002 \\
\hline 15 & 115 IC5 & Y & -0.0764 & 0 & 0.0002 & 0.0002 \\
\hline 16 & 116 IC5 & Y & -0.0764 & 0 & 0.0002 & 0.0002 \\
\hline 17 & 117 IC5 & Y & -0.0764 & 0 & 0.0002 & 0.0002 \\
\hline 18 & 118 IC5 & Y & -0.0764 & 0 & 0.0002 & 0.0002 \\
\hline 19 & 119 IC5 & Y & -0.0764 & 0 & 0.0002 & 0.0002 \\
\hline 20 & 120 IC5 & Y & -0.0764 & 0 & 0.0002 & 0.0002 \\
\hline 21 & 101 IC9 & Y & 0.0681 & 0 & 0.0002 & 0.0002 \\
\hline 22 & 102 IC9 & Y & 0.0681 & 0 & 0.0002 & 0.0002 \\
\hline 23 & 103 IC9 & Y & 0.0681 & 0 & 0.0002 & 0.0002 \\
\hline 24 & 104 IC9 & Y & 0.0681 & 0 & 0.0002 & 0.0002 \\
\hline 25 & $105 \mathrm{IC} 9$ & Y & 0.0681 & 0 & 0.0002 & 0.0002 \\
\hline 26 & 106 IC9 & Y & 0.0681 & 0 & 0.0002 & 0.0002 \\
\hline 27 & 107 IC9 & Y & 0.0681 & 0 & 0.0002 & 0.0002 \\
\hline 28 & 108 IC9 & Y & 0.0681 & 0 & 0.0002 & 0.0002 \\
\hline 29 & 109 IC9 & Y & 0.0681 & 0 & 0.0002 & 0.0002 \\
\hline 30 & $110 \mathrm{IC9}$ & Y & 0.0681 & 0 & 0.0002 & 0.0002 \\
\hline 31 & 111 IC9 & Y & 0.0681 & 0 & 0.0002 & 0.0002 \\
\hline 32 & 112 IC9 & Y & 0.0681 & 0 & 0.0002 & 0.0002 \\
\hline & $\begin{array}{l}\text { 11n In } \\
\text { Sheet1 }\end{array}$ & Y̌shee & & & $\mathrm{AnOMn}$ & ก ח ח \\
\hline
\end{tabular}




\subsection{Output}

The output from the software tool contains all feature control plots, which are explained in detail below, and a spreadsheet comprised of raw data with all feature finish process calculations, $C_{p}$ and $C_{p k}$ calculations at forging stage and $\mathrm{C}_{\mathrm{pk}}$ calculations after all finish processing. The raw data spreadsheet table is as shown in the Table 5-7; it consists of a raw data where all the forging to final calculations are compiled, a $\mathrm{C}_{\mathrm{p}}$ and $\mathrm{C}_{\mathrm{pk}}$ calculation sheet as shown in Table 5-8 and $\mathrm{C}_{\mathrm{pk}}$ values after final processing as shown in Table 5-9.

The chart type used in the tool is a run chart with process capability indices added to it. These run charts have different sections (A through G) plotted for the same feature on a single plot, and each plot has the control limits calculated for each section, which is atypical of a run chart. Each chart consists of observation number on the $x$-axis and deviation from the nominal on the y-axis. The upper and lower specification limits that are taken from the blue print requirements plotted in blue colored lines [Note: The specification limits on certain features (XXX, YYY, LEP etc) are different for various cross sections]. The nominal value of the feature is plotted in Teal colored line. The upper and lower control limits calculated from the spread within the data are plotted in Red colored lines. The mean value of the data is represented by the purple colored line. The black dots represent the actual observations for each section that is a non-reference section, and yellow dots are for information only, not for product acceptance. The output screen shots for features are shown from Figures 5-3 to 5-31. 
Table 5-7: Raw data forging to final calculations

\begin{tabular}{|c|c|c|c|c|c|c|c|c|c|c|c|c|c|}
\hline 4 & $\mathrm{BX}$ & BY & $\mathrm{BZ}$ & $\mathrm{CA}$ & $\mathrm{CB}$ & $\mathrm{CC}$ & CD & CE & CF & CG & $\mathrm{CH}$ & $\mathrm{Cl}$ & CJ \\
\hline 1 & Sect & Offset & Closure & Pn1 Mean & Plea1 Mean & Ptea1 Mean & Pn1 & Plea1 & Ptea1 & & LET_Final & TET_Final & MXT_Final \\
\hline 2 & A & -3 & -1 & -4 & 0.805 & 7.9815 & -5.7435 & -11.7135 & 7.1965 & & $\overline{-0} .0012$ & $\overline{0} .0005$ & $\overline{-0} .0017$ \\
\hline 3 & $B$ & -3 & -1.352 & -3.6675 & 4.361 & 6.7635 & -4.6635 & 2.7565 & 6.0265 & & -0.0004 & -0.0005 & -0.0009 \\
\hline 4 & C & -3 & -2.3796 & 2.4144 & 7.7574 & 11.1169 & -0.9935 & 5.4765 & 9.8265 & & -0.0004 & -0.0006 & 0 \\
\hline 5 & D & -3 & -3.9582 & 2.8043 & 5.5103 & 8.8498 & -2.7035 & 3.8865 & 8.9265 & & -0.0001 & 0.0011 & 0.0011 \\
\hline 6 & $E$ & -3 & -6.0878 & -0.4853 & 3.2357 & 8.6357 & 0.6165 & 7.1665 & 11.0265 & & 0.0002 & -0.0013 & -0.0007 \\
\hline 7 & $\mathrm{~F}$ & -3 & -8.7684 & -3.2679 & -2.4374 & 6.8441 & -6.3835 & -0.1435 & 6.6665 & & -0.0017 & -0.0007 & 0 \\
\hline 8 & G & -3 & -12 & 4.476 & 2.869 & 11.96 & -9.6835 & -2.9235 & 5.2465 & & -0.0005 & 0.0008 & -0.0027 \\
\hline 9 & & & & & & & 0.1665 & -5.8235 & 11.4365 & & 0.0003 & 0.0004 & -0.0001 \\
\hline 10 & & & & & & & -0.9935 & 5.0965 & 10.1065 & & -0.0008 & -0.0006 & -0.0003 \\
\hline 11 & & & & & & & -12.2735 & -5.8935 & 0.6565 & & -0.0015 & 0.0012 & -0.001 \\
\hline 12 & & & & & & & -10.9235 & -4.7735 & 5.2865 & & -0.0013 & -0.0005 & -0.0015 \\
\hline 13 & & & & & & & -0.6935 & 5.7165 & 11.4965 & & -0.0016 & -0.0004 & 0.0001 \\
\hline 14 & & & & & & & -1.2035 & 6.5065 & 9.9165 & & -0.0012 & 0.0001 & -0.0011 \\
\hline 15 & & & & & & & -7.8235 & -1.0035 & 4.5065 & & -0.0002 & 0.0003 & -0.0007 \\
\hline 16 & & & & & & & 0.5865 & 6.7965 & 11.5565 & & -0.001 & -0.0004 & -0.0002 \\
\hline 17 & & & & & & & -2.4335 & 5.4465 & 8.7365 & & -0.0015 & -0.001 & -0.0018 \\
\hline 18 & & & & & & & -5.3835 & 1.6265 & 5.8565 & & -0.0006 & 0.0004 & -0.0005 \\
\hline 19 & & & & & & & -4.4235 & 1.9865 & 6.4365 & & -0.0004 & -0.0007 & 0.0002 \\
\hline 20 & & & & & & & -3.4735 & -9.4135 & 9.3465 & & 0.0011 & -0.0005 & -0.0015 \\
\hline 21 & & & & & & & -1.5735 & 5.3265 & 9.3765 & & 0.0003 & 0.0002 & 0.0004 \\
\hline 22 & & & & & & & -7.9955 & -1.0155 & 7.6445 & & -0.0002 & 0.0003 & -0.0016 \\
\hline 23 & & & & & & & -4.5355 & 6.2245 & 3.5945 & & 0.0007 & -0.0005 & -0.0014 \\
\hline 24 & & & & & & & 0.1045 & 6.3745 & 9.6245 & & 0.0011 & -0.0015 & -0.0018 \\
\hline 25 & & & & & & & -3.9355 & 4.5345 & 5.8845 & & 0.0011 & 0.0003 & 0.0002 \\
\hline 26 & & & & & & & 1.1045 & 8.9545 & 6.1445 & & 0.0009 & -0.002 & -0.0014 \\
\hline 27 & & & & & & & -6.7955 & 1.5945 & 8.3045 & & -0.0009 & -0.0011 & -0.0007 \\
\hline 28 & & & & & & & -4.6255 & 6.2145 & 6.2145 & & 0.0012 & 0.0004 & -0.0023 \\
\hline 29 & & & & & & & 2.2845 & 6.3545 & 7.1145 & & 0.0005 & -0.0021 & -0.0016 \\
\hline 30 & & & & & & & -3.5555 & 3.5445 & 7.9245 & & -0.0006 & -0.0021 & -0.0009 \\
\hline 31 & & & & & & & -7.4955 & -1.2655 & 6.2545 & & -0.0008 & -0.0004 & -0.0019 \\
\hline 32 & & & & & & & -7.6555 & -1.5755 & 11.6345 & & -0.001 & -0.0017 & -0.0018 \\
\hline 27 & $\rightarrow 1$ & Raw Data & Cp-Cpk a & IP $<$ CPK a & FINAL $<$ & & n nדer & croar & CAcar & & Annna & nnnar & ก nnกn \\
\hline
\end{tabular}


Table 5-8: $\mathrm{C}_{\mathrm{p}}$ and $\mathrm{C}_{\mathrm{pk}}$ calculations at IP (In-Process)

\begin{tabular}{|c|c|c|c|c|c|c|c|c|c|c|}
\hline 4 & A & B & C & D & $E$ & $F$ & G & $\mathrm{H}$ & 1 & $\mathrm{~J}$ \\
\hline 1 & $\mathrm{xxx}$ & YYY & $\mathrm{C}$ & $\mathrm{N}$ & LEA & TEA & LET & TET & IXXT & $\mathbb{P}$ \\
\hline 2 & 2.4 & 2.8 & 1.4 & 2.2 & 1.4 & 2.9 & 1.6 & 1.7 & 1.3 & C. \\
\hline 3 & 4.3 & 4.5 & 1.7 & 3.0 & 2.9 & 3.7 & 1.6 & 1.5 & 1.8 & C. \\
\hline 4 & 8.4 & 5.2 & 3.9 & 3.4 & 2.8 & 2.9 & 2.2 & 1.8 & 3.1 & C. \\
\hline 5 & 9.0 & 4.3 & 3.2 & 3.7 & 3.0 & 3.1 & 2.7 & 1.9 & 2.5 & C. \\
\hline 6 & 9.1 & 4.3 & 3.3 & 4.4 & 5.0 & 3.0 & 2.9 & 2.4 & 2.5 & C. \\
\hline 7 & 9.3 & 4.8 & 4.4 & 4.2 & 3.6 & 3.2 & 4.2 & 2.8 & 2.8 & C. \\
\hline 8 & 9.6 & 5.2 & 3.9 & 3.7 & 3.6 & 2.9 & 4.0 & 2.6 & 2.8 & C. \\
\hline 9 & & & & & & & & & & \\
\hline 10 & 1.4 & 2.2 & 1.3 & 2.2 & 1.2 & 1.5 & 1.5 & 1.4 & 1.3 & $C_{-k}$ \\
\hline 11 & 2.7 & 2.9 & 1.5 & 2.9 & 2.1 & 2.4 & 1.2 & 1.4 & 1.6 & $C_{-k}$ \\
\hline 12 & 5.5 & 2.7 & 3.5 & 2.9 & 1.8 & 1.4 & 1.5 & 1.6 & 3.0 & $C_{-k}$ \\
\hline 13 & 6.8 & 2.3 & 2.8 & 3.3 & 2.3 & 2.0 & 2.1 & 1.8 & 2.1 & $C_{-k}$ \\
\hline 14 & 6.6 & 2.0 & 2.4 & 4.2 & 4.5 & 2.1 & 2.9 & 1.7 & 1.9 & $C_{-k}$ \\
\hline 15 & 7.0 & 2.6 & 3.7 & 3.6 & 3.3 & 2.3 & 4.0 & 1.9 & 1.5 & $C_{-k}$ \\
\hline 16 & 7.2 & 3.1 & 3.5 & 3.1 & 3.2 & 1.5 & 2.7 & 2.2 & 1.4 & $C_{-k}$ \\
\hline
\end{tabular}

Table 5-9: $\mathrm{C}_{\mathrm{pk}}$ at final processing

\begin{tabular}{|c|c|c|c|c|c|c|c|c|}
\hline & A & B & C & D & E & F & G & H \\
\hline 1 & C & N & LEA & TEA & LET & TET & MXT & Final \\
\hline 2 & 1.2 & 3.6 & 2.6 & 4.3 & 1.7 & 1.5 & 1.3 & $C_{p k}$ \\
\hline 3 & 1.5 & 5.0 & 4.7 & 5.7 & 2.0 & 1.2 & 1.5 & $C_{p k}$ \\
\hline 4 & 3.4 & 5.7 & 4.1 & 3.9 & 2.6 & 1.7 & 2.8 & $C_{p k}$ \\
\hline 5 & 2.8 & 6.2 & 4.7 & 4.5 & 3.4 & 1.8 & 2.3 & $C_{p k}$ \\
\hline 6 & 3.3 & 7.9 & 8.3 & 4.4 & 3.0 & 1.9 & 2.1 & $C_{p k}$ \\
\hline 7 & 4.0 & 7.0 & 6.2 & 4.8 & 4.7 & 2.2 & 1.7 & $C_{p k}$ \\
\hline 8 & 3.2 & 6.0 & 6.1 & 3.9 & 4.8 & 2.4 & 1.6 & $C_{p k}$ \\
\hline
\end{tabular}


As shown in the Figure 5-3, the XXX feature plotted has a USL and LSL that are different for each section hence they are staggered (represented by the blue lines) as opposed to a single line. The deviations from the nominal values are reported by the CMM, and these are plotted for each section (represented by black dots). The red lines above and below the data measurements are the UCL and LCL calculated using the equation 16. The plot features are identical for all features except for those specified clearly.

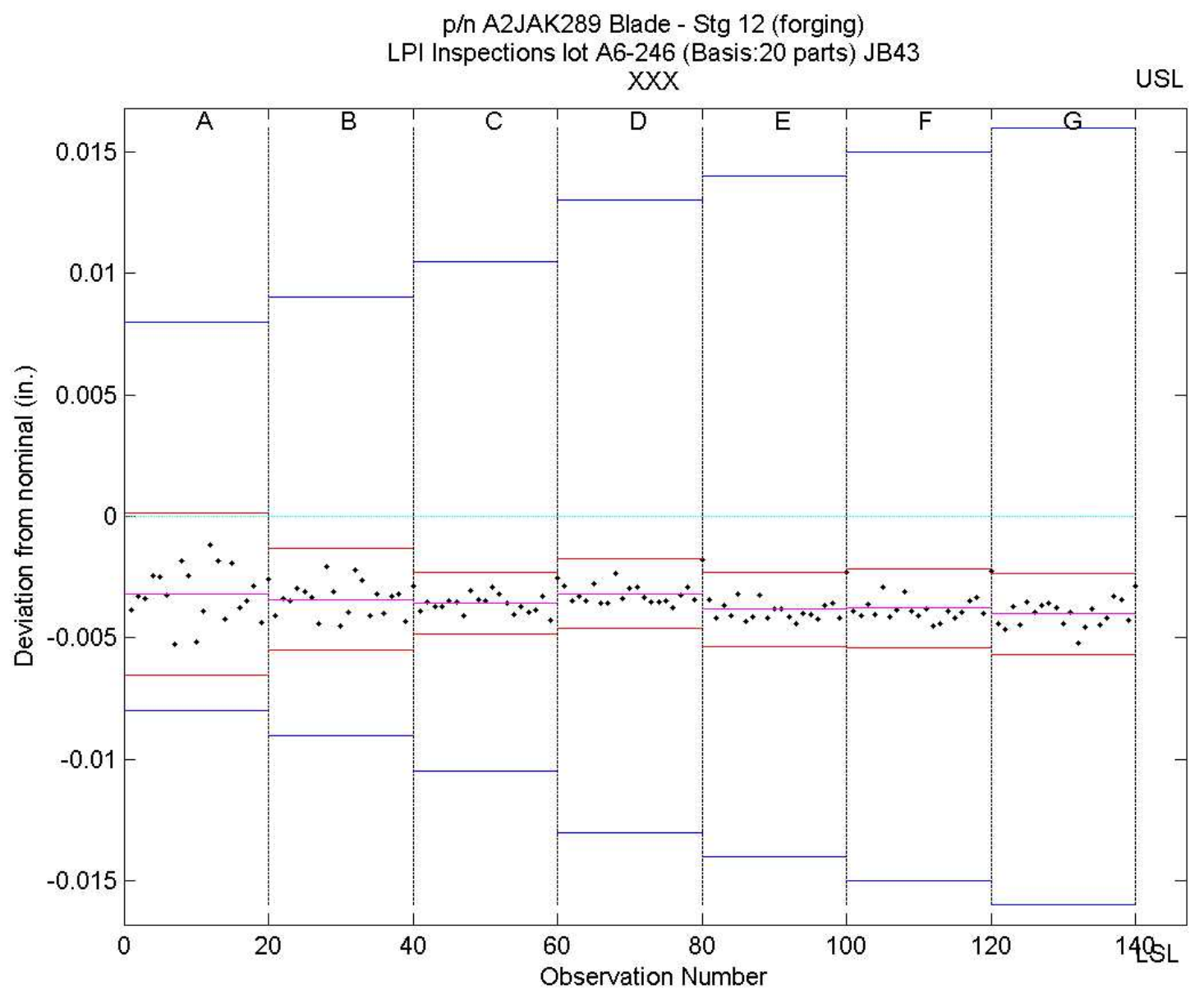

Figure 5-3: XXX Section A-G 
As shown in Figure 5-4, the DTPX (Delta True Position for XXX) was calculated using Equation 1. The deviation difference for a given section and its immediate adjacent sections are plotted. The USL and LSL are identical for each section calculation. The UCL and LCL were calculated using Equation 16.

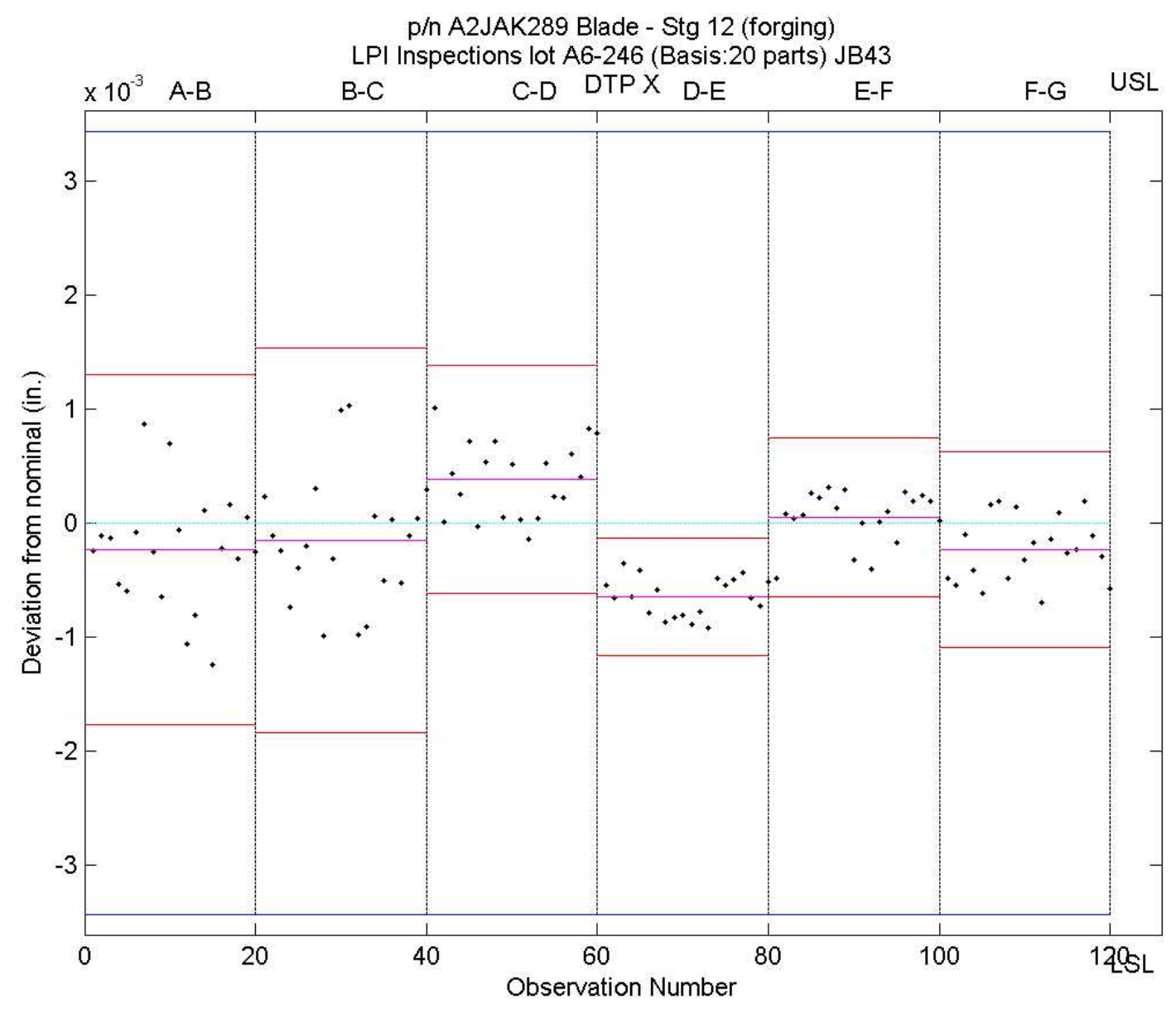

Figure 5-4: DTPX 
As shown in the Figure 5-5, the YYY feature calculations and plots are same as the XXX feature. The USL and LSL are different for each section hence they are staggered (represented by the blue lines) as opposed to a single line. The plot features are identical to other plots.

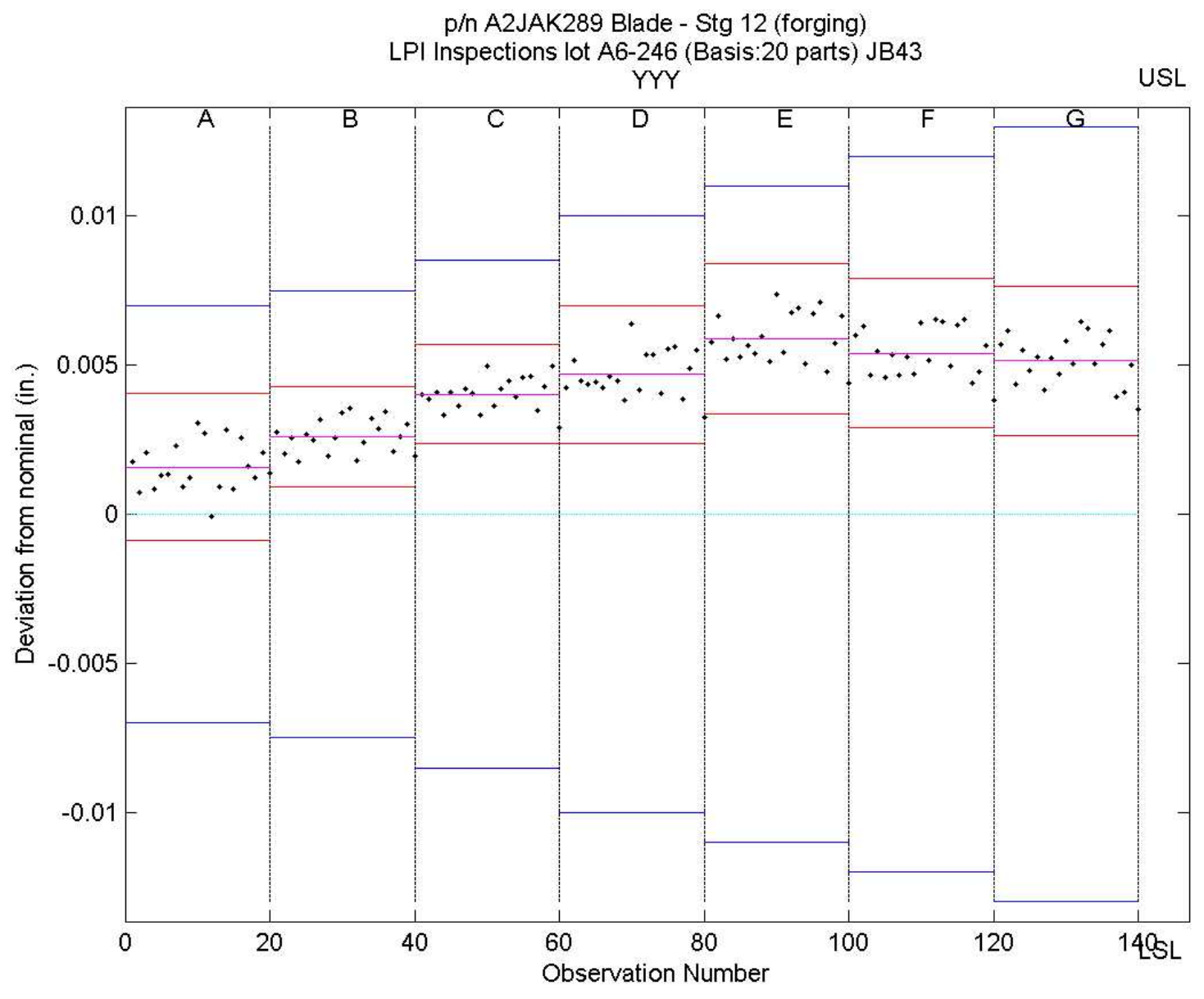

Figure 5-5: YYY Section A-G 
As shown in Figure 5-6, the DTPY (Delta True Position for YYY) was calculated using Equation 1 the same way DTPX is calculated, the deviation difference for a given section and its immediate adjacent sections are plotted. The USL and LSL are identical for each section calculation.

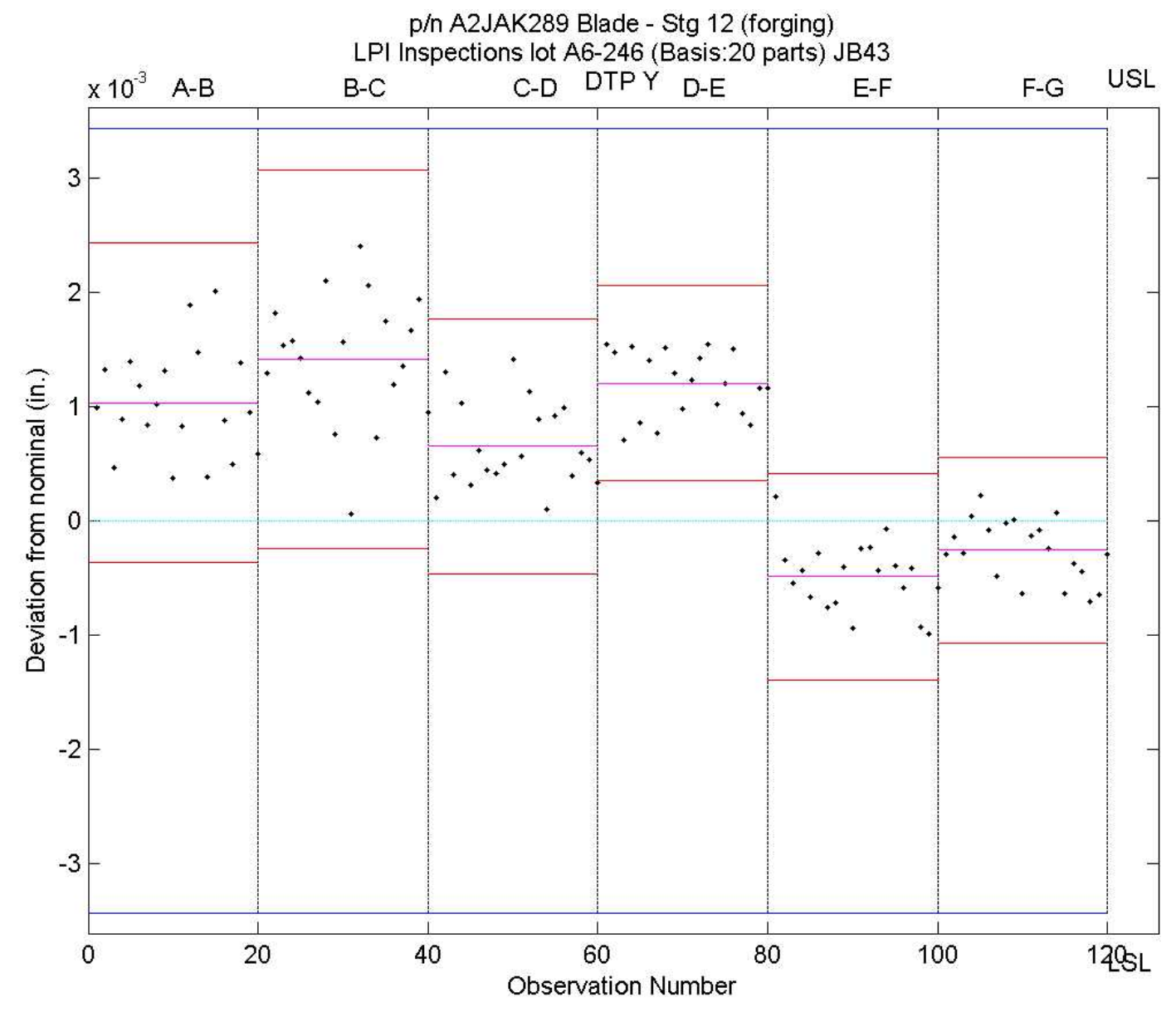

Figure 5-6: DTPY 
As shown in Figure 5-7, the chord values are plotted for each section. The UCL and LCL were calculated using Equation 16 and plot features are identical to other plots.

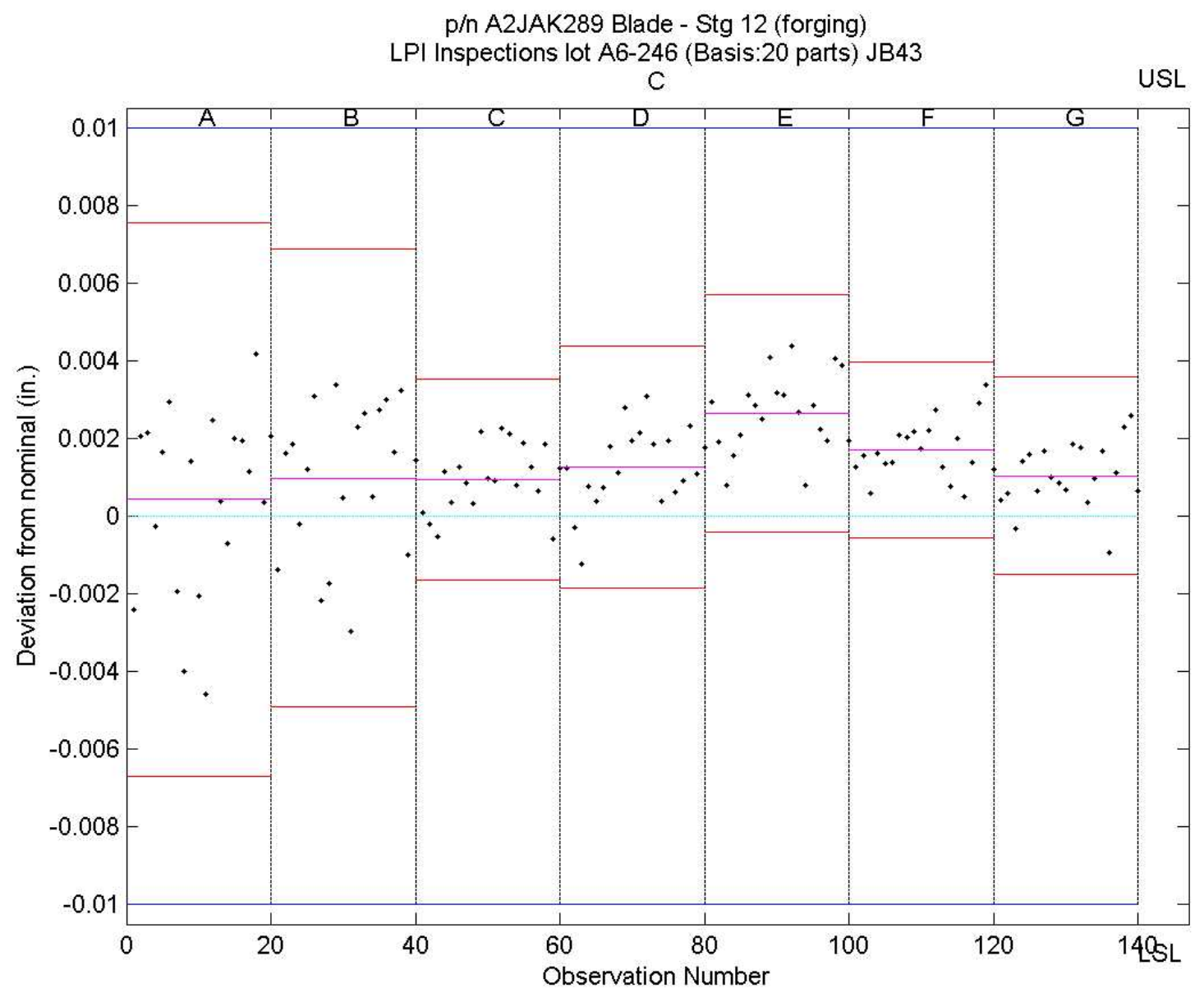

Figure 5-7: Chord Section A-G 
As shown in Figure 5-8, the chord final calculations were calculated using Equation 3after final processing; the USL and LSL are identical for each section. All the other plot features are identical to the other plots.

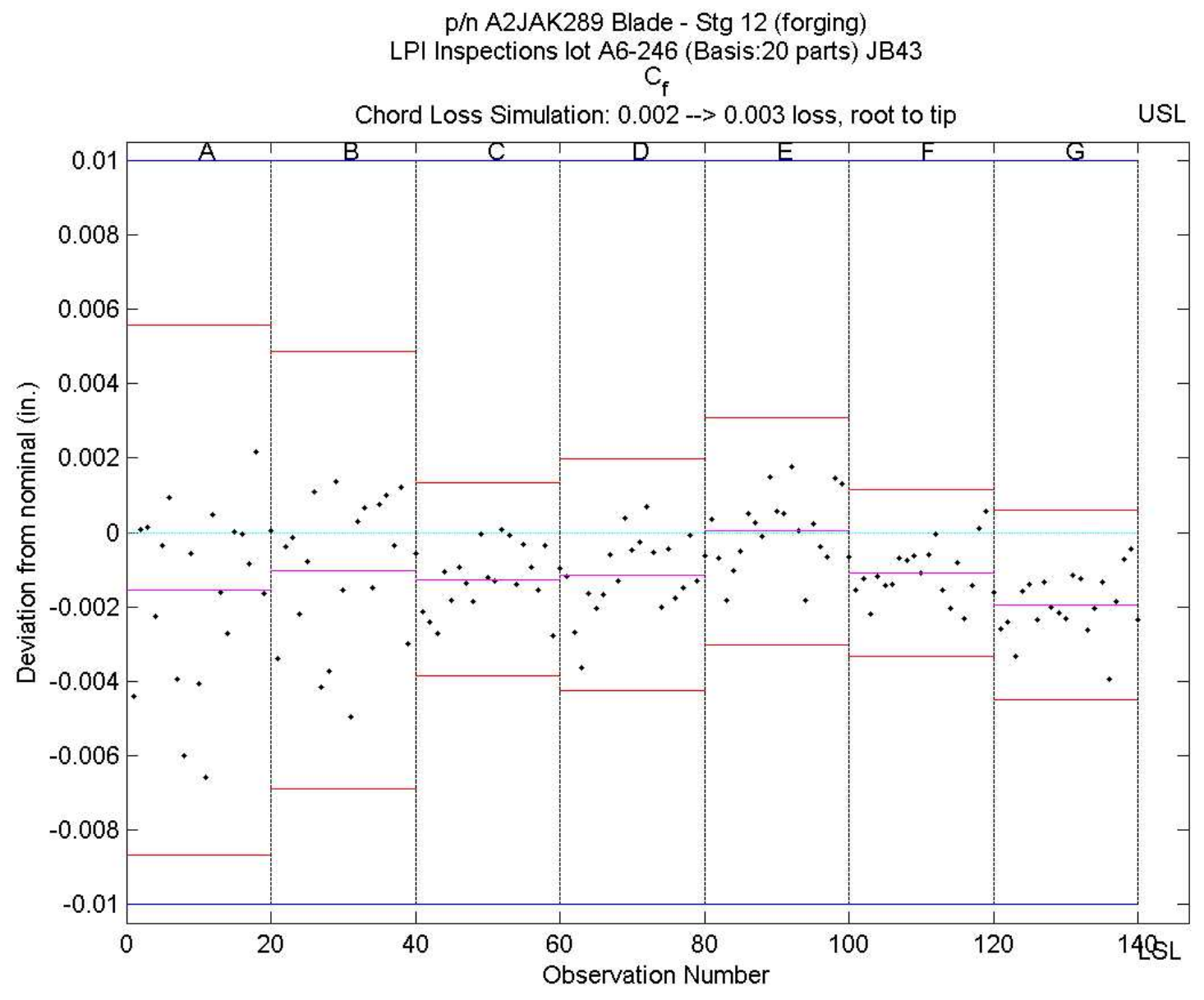

Figure 5-8: Chord Final Section A-G 
As shown in Figure 5-9, the N-angle plot has the data centered to section A to assist the operator with better N-angle offset targeting. The USL and LSL values are different for each section, hence they are staggered. The UCL and LCL were calculated using Equation 16 and all other plot features are identical to the other plots.

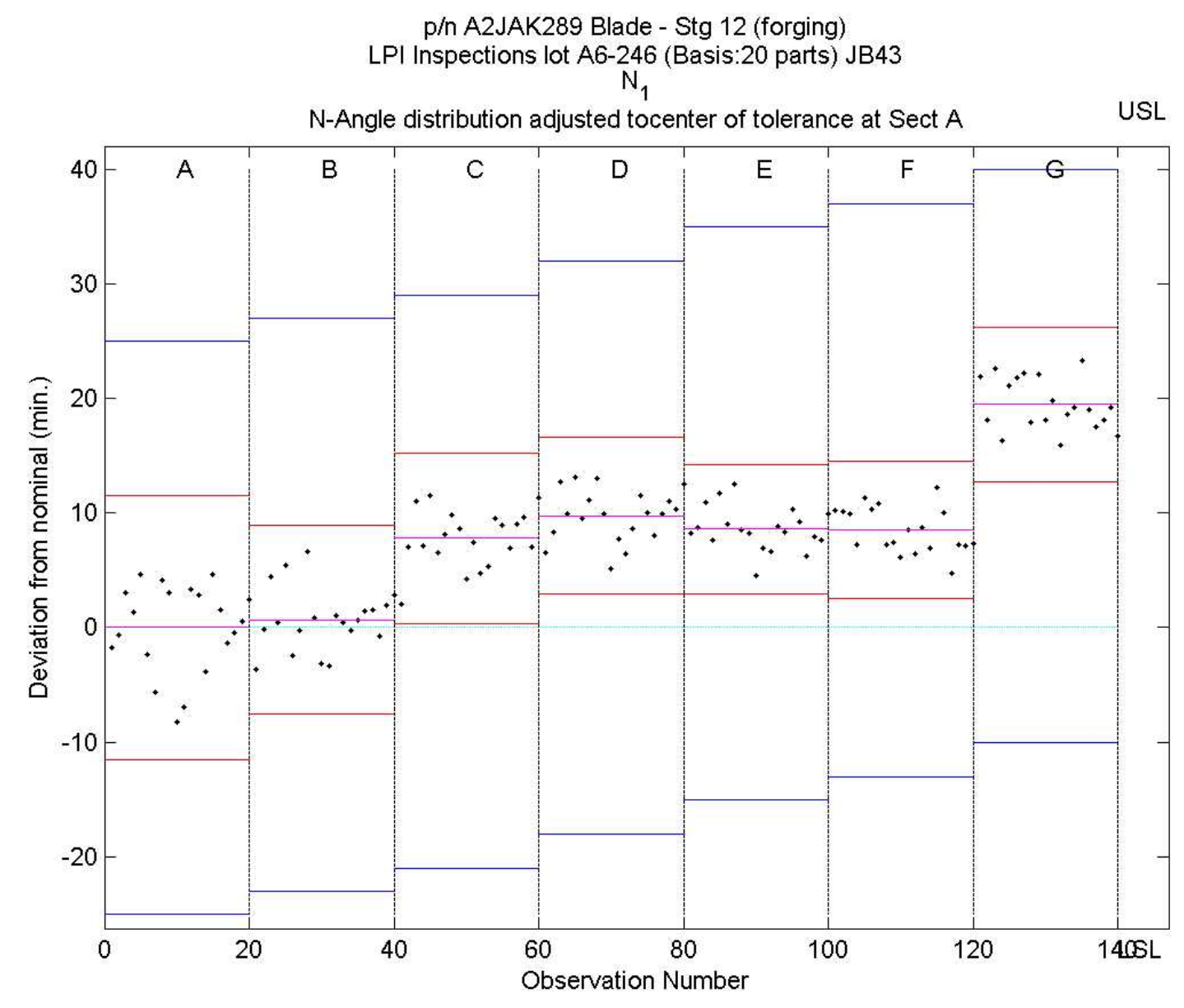

Figure 5-9: N-angle (pre-peen) Section A-G 
As shown in Figure 5-10, the DTPN was calculated using Equation 1 similar to DTPX and DTPY, USL and LSL are identical for all sections.

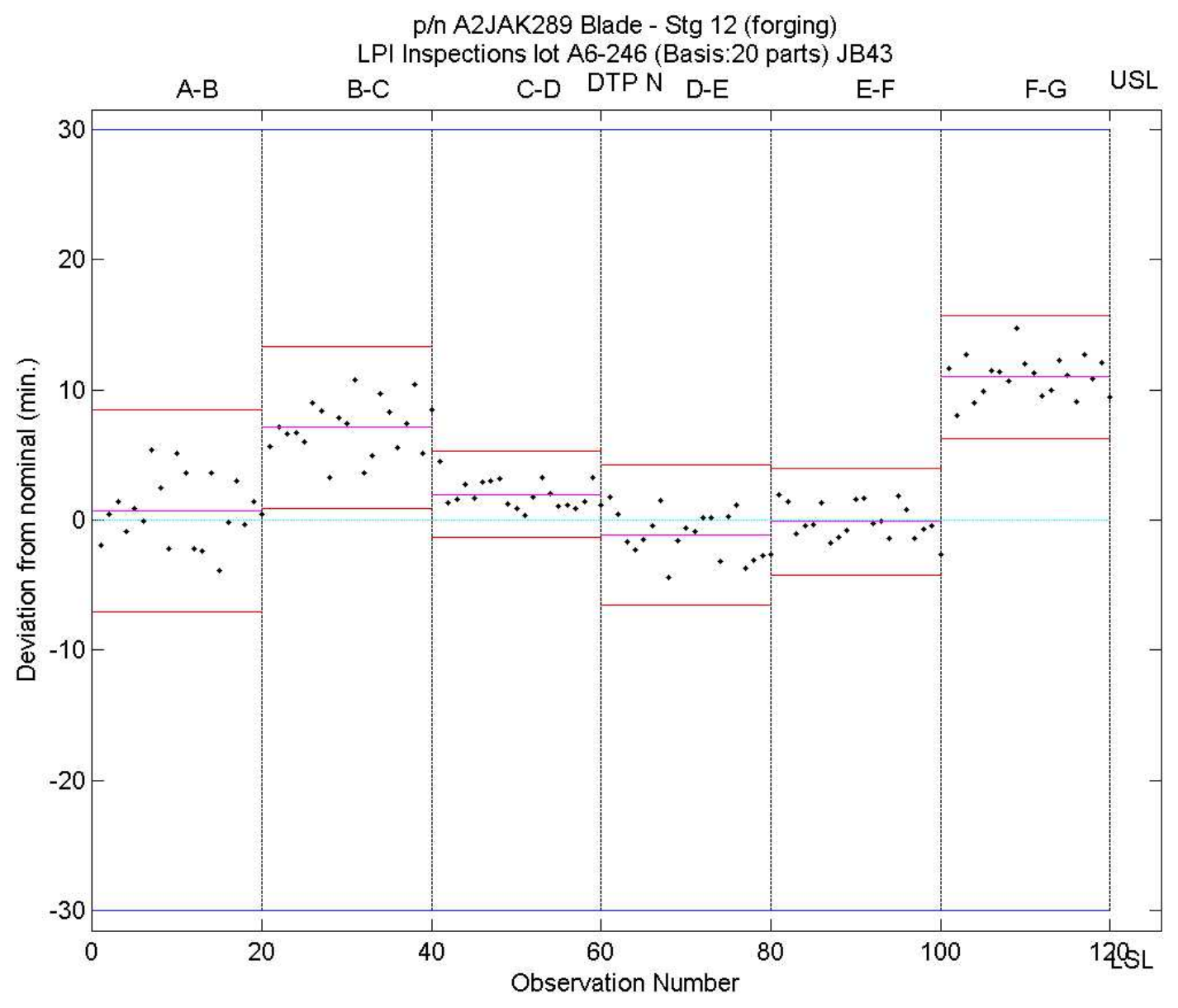

Figure 5-10: DTPN 
As shown in Figure 5-11, the LEA plot has the data centered to section A to assist the operator with better $\mathrm{N}$-angle offset targeting, as $\mathrm{N}$-angle controls LEA and TEA. Section 'A' data points are colored in yellow since it is a reference section per design. The USL and LSL values are different for each section, hence they are staggered. The UCL and LCL were calculated using Equation 16 and all other plot features are identical to the other plots.

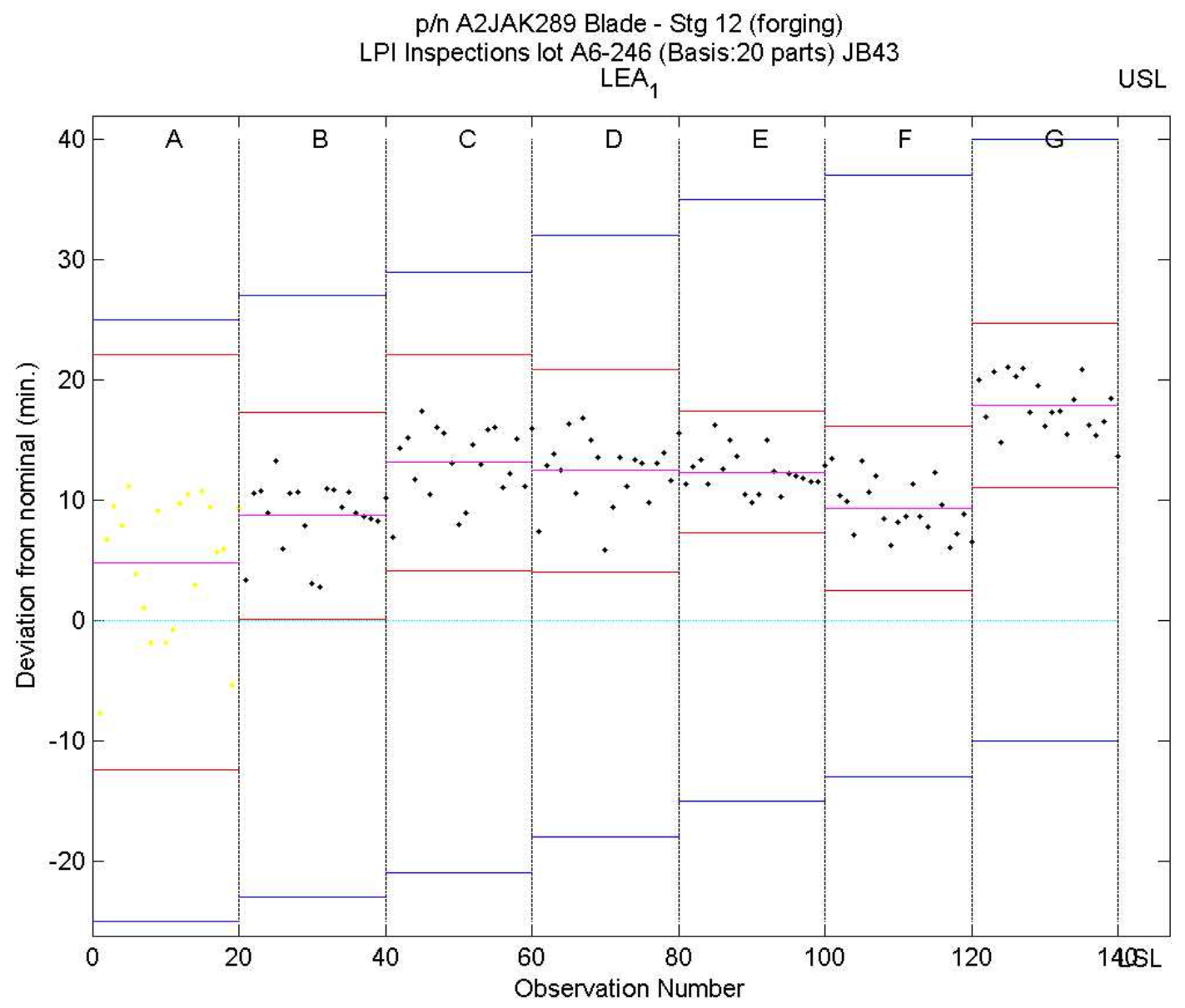

Figure 5-11: Leading Edge Angle (pre-peen) Section A-G 
As shown in Figure 5-12, the CLEA (Camber Leading Edge Angle) is for information only, it is not a product requirement.

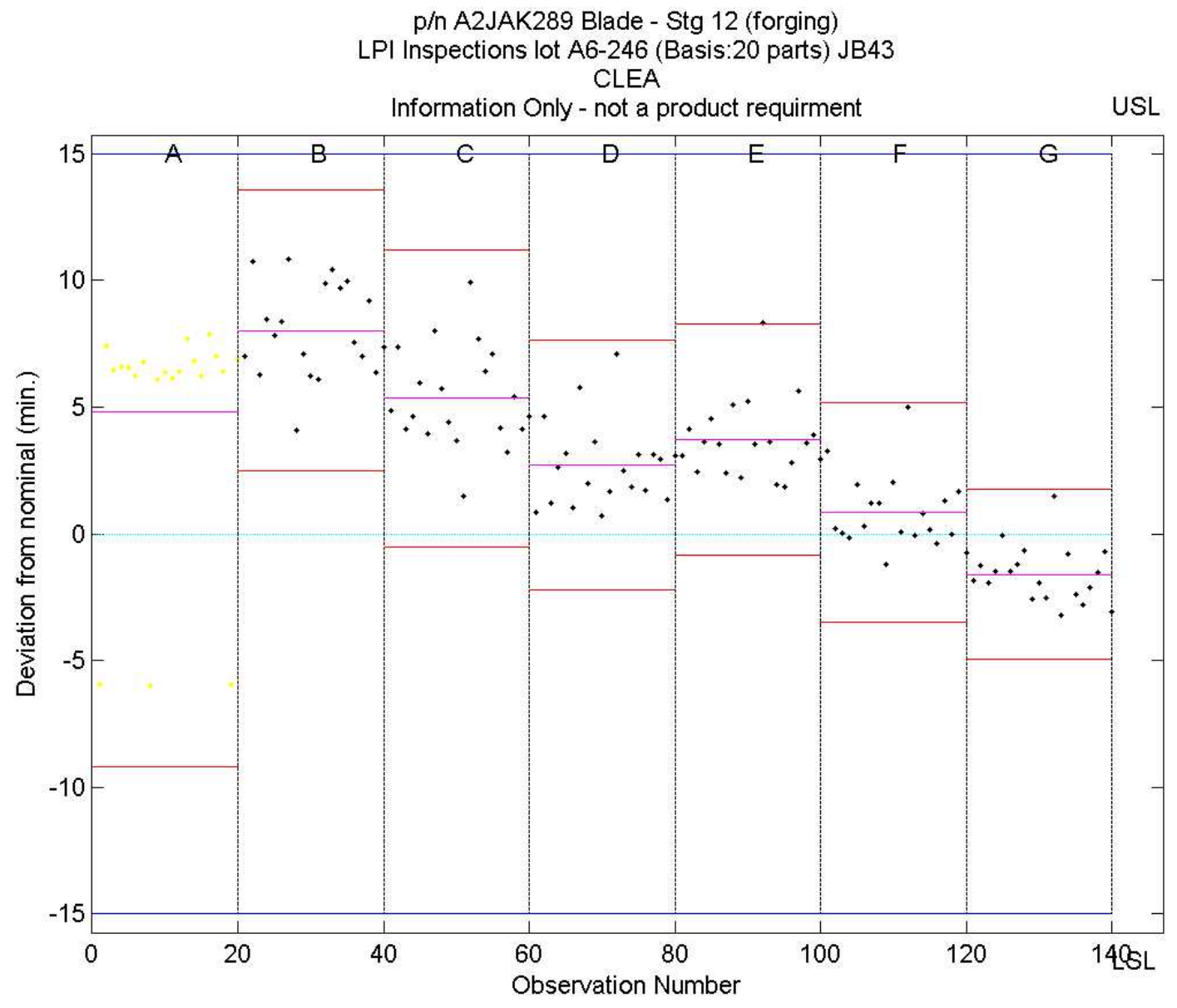

Figure 5-12: CLEA (Camber LEA)- Information only 
As shown in Figure 5-13, the TEA plot has the data centered to section A to assist the operator with better $\mathrm{N}$-angle offset targeting, as $\mathrm{N}$-angle controls LEA and TEA. Section 'A' data points are colored in yellow since it is a reference section per design. The USL and LSL values are different for each section, hence they are staggered. The UCL and LCL were calculated using equation 16 and all other plot features are identical to the other plots.

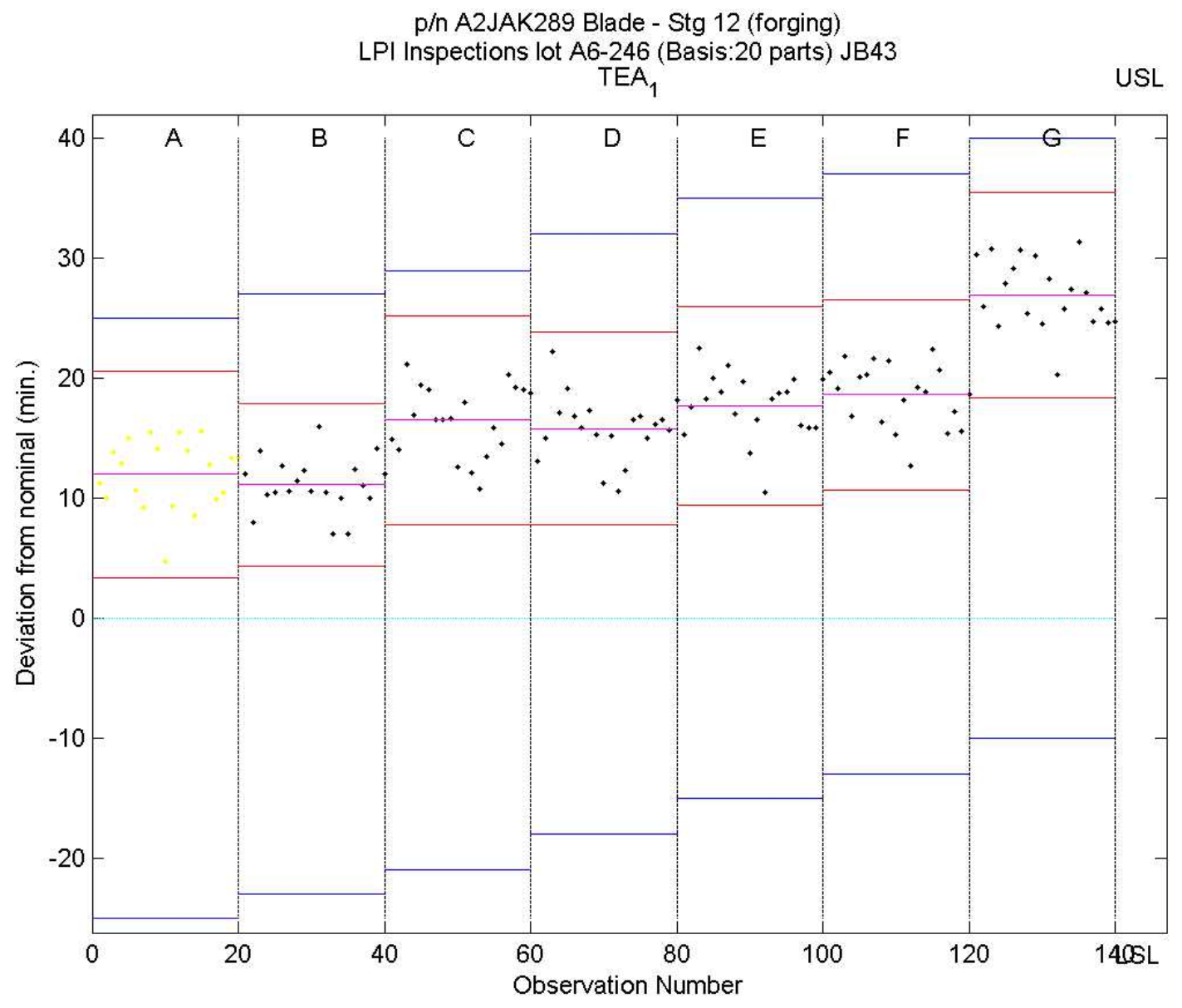

Figure 5-13: Trailing Edge Angle (pre-peen) Section A-G 
As shown in Figure 5-14, the CTEA (Camber Trailing Edge Angle) is for information only, it is not a product requirement.

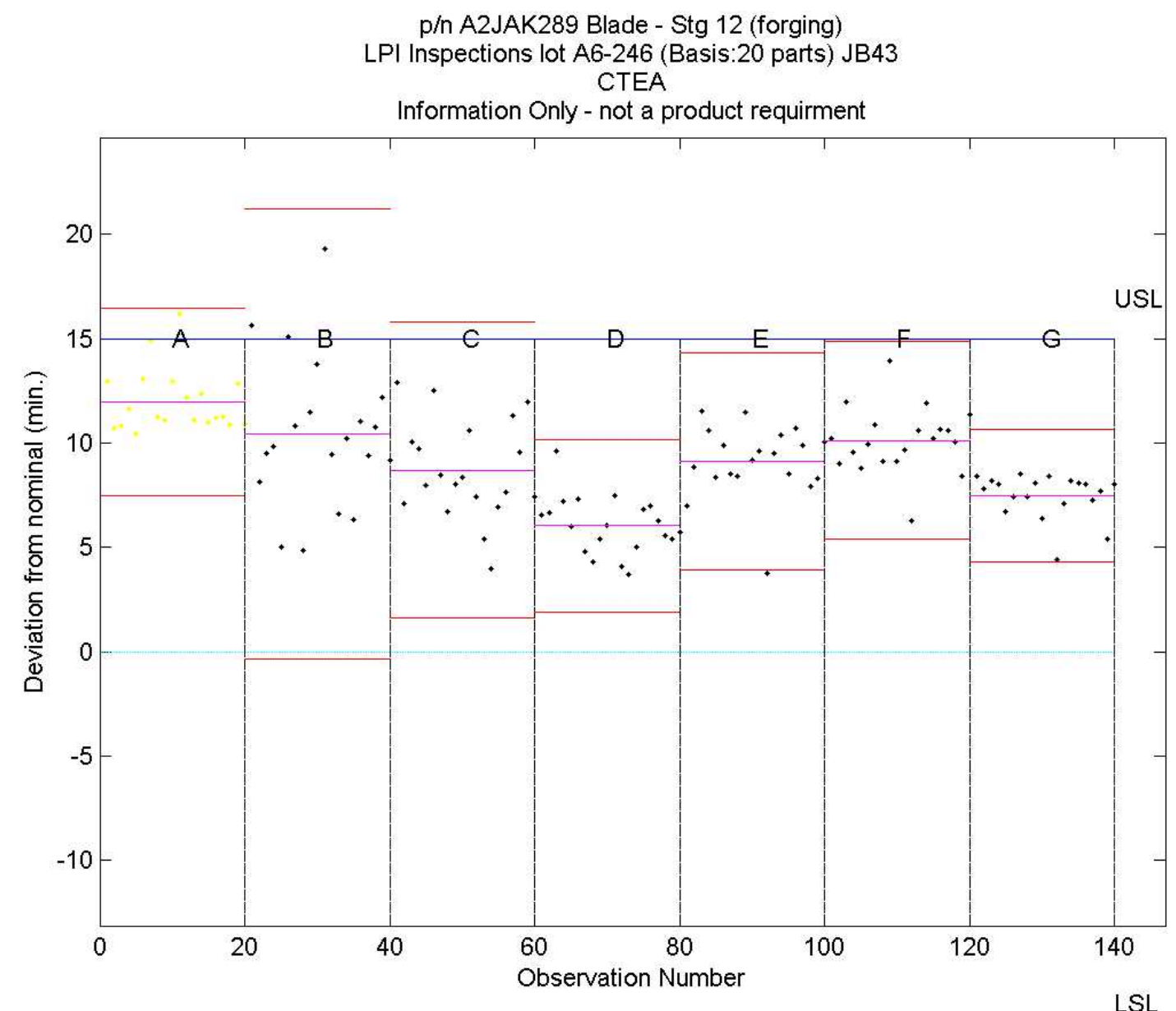

Figure 5-14: CTEA (Camber TEA)- Information only 
As shown in Figure 5-15, the LEP (Leading Edge Profile) is plotted similar to other features. The UCL and LCL were calculated using Equation 16. The USL is identical for all sections except for Section 'A'; hence they are staggered for that section. Because this is a one sided plot, the LSL is zero.

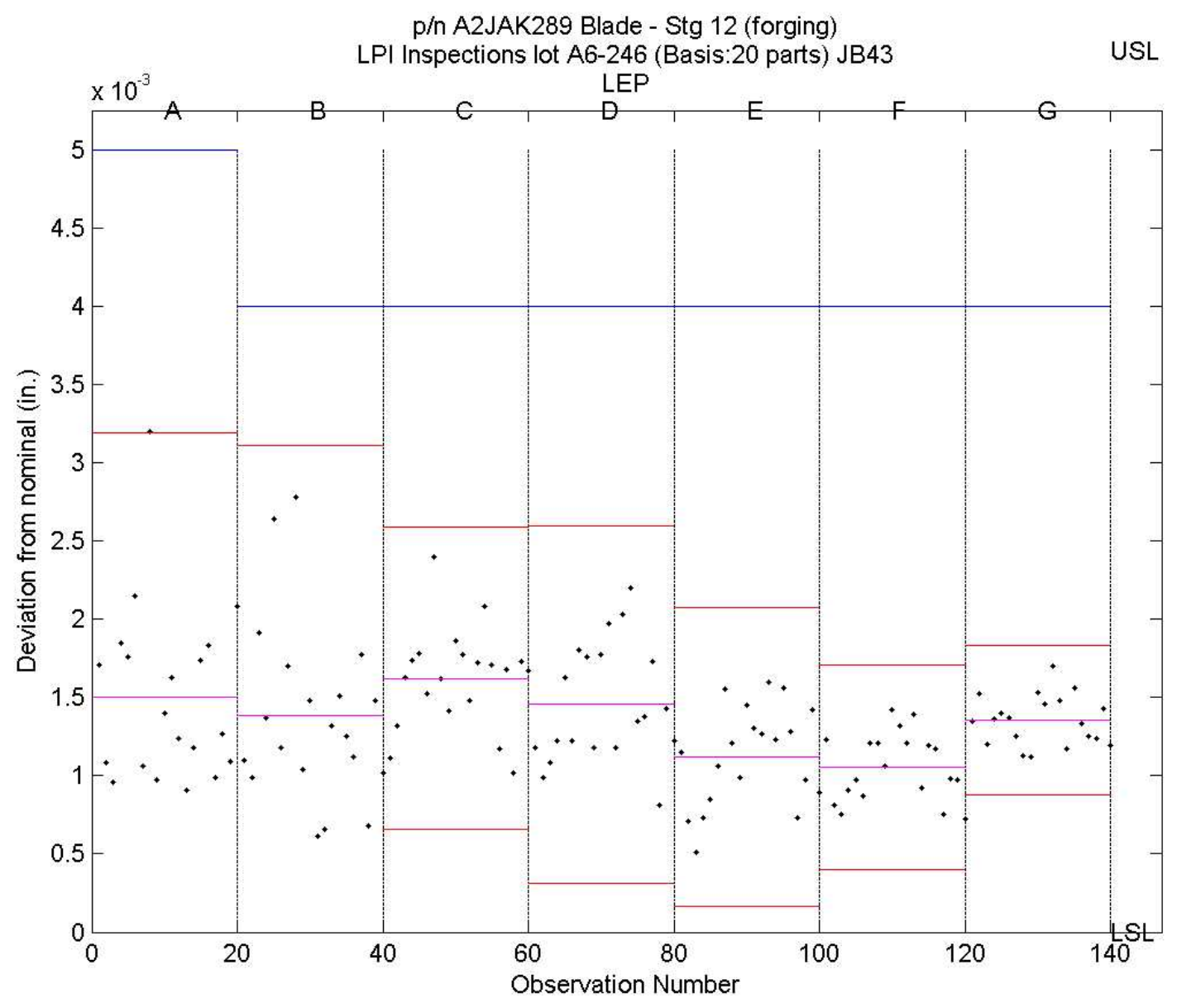

Figure 5-15: Leading Edge Profile Section A-G 
As shown in Figure 5-16, the TEP (Trailing Edge Profile) is plotted similar to other features. The UCL and LCL were calculated using Equation 16. The USL and LSL are identical for all sections except for Section 'A'; hence they are staggered for that section. Because this is a one sided plot, the LSL is zero.

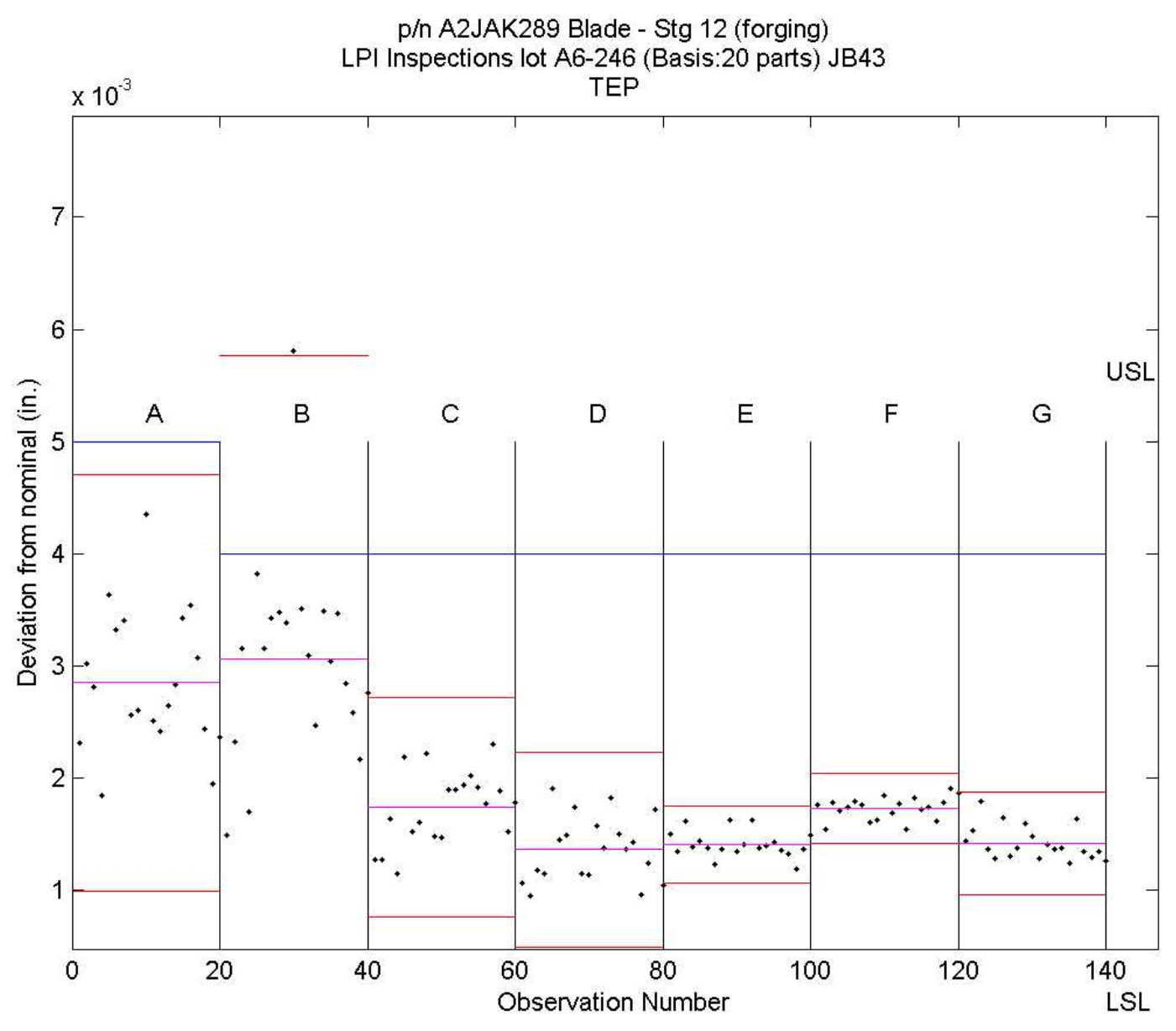

Figure 5-16: Trailing Edge Profile Section A-G 
As shown in Figure 5-17, the PSP (Pressure Side Profile) is plotted similar to other features. The UCL and LCL were calculated using Equation 16. The USL and LSL are identical for all sections. Because this is a one sided plot, the LSL is zero.

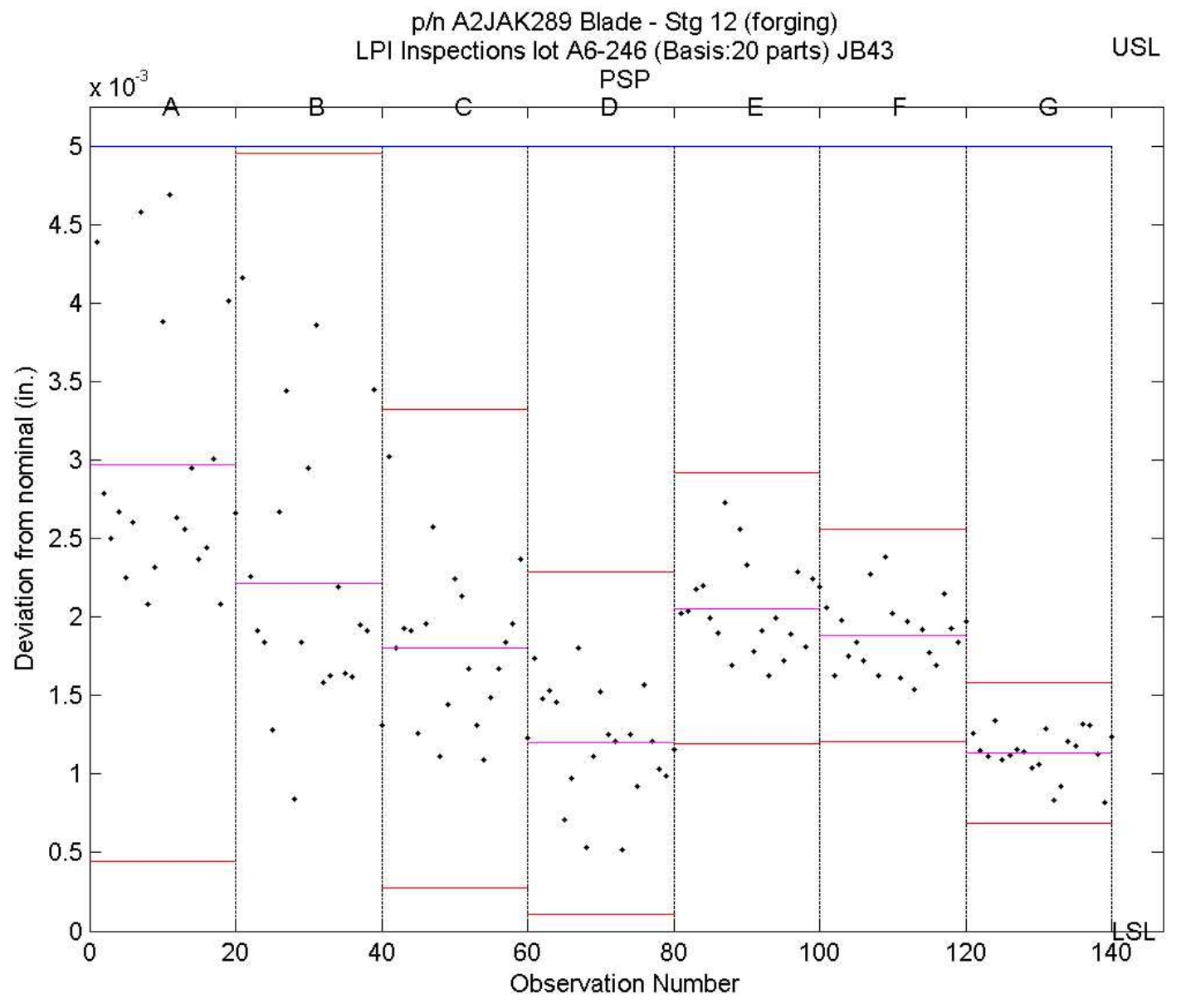

Figure 5-17: Pressure Side Profile Section A-G 
As shown in Figure 5-18, the SSP (Suction Side Profile) is plotted similar to other features. The UCL and LCL were calculated using Equation 16. The USL and LSL are identical for all sections. Because this is a one sided plot, the LSL is zero.

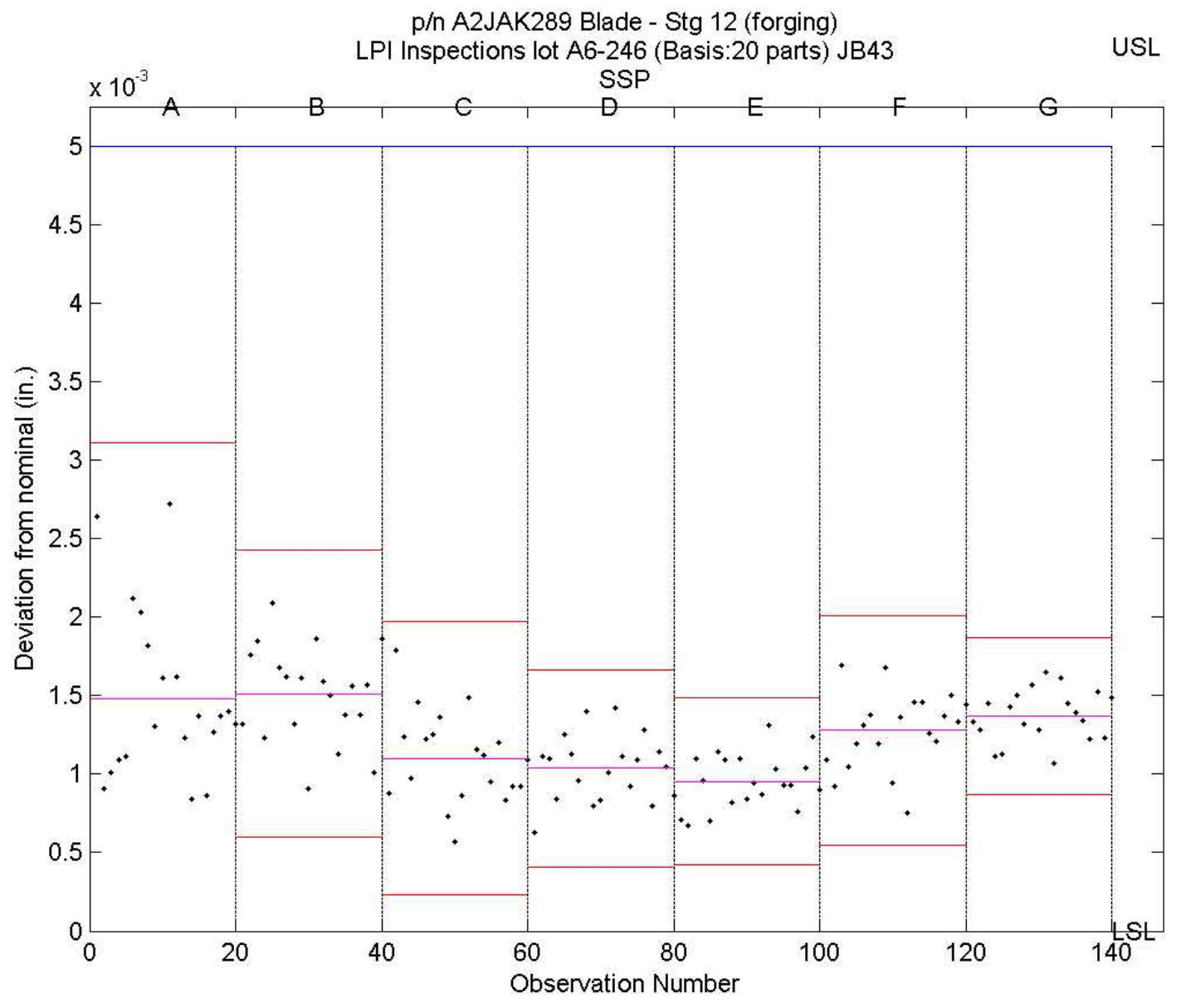

Figure 5-18: Suction Side Profile Section A-G 
As shown in Figure 5-19, the LET (Leading Edge Thickness) is plotted similar to other features. The UCL and LCL were calculated using Equation 16. The USL and LSL are identical for all sections.

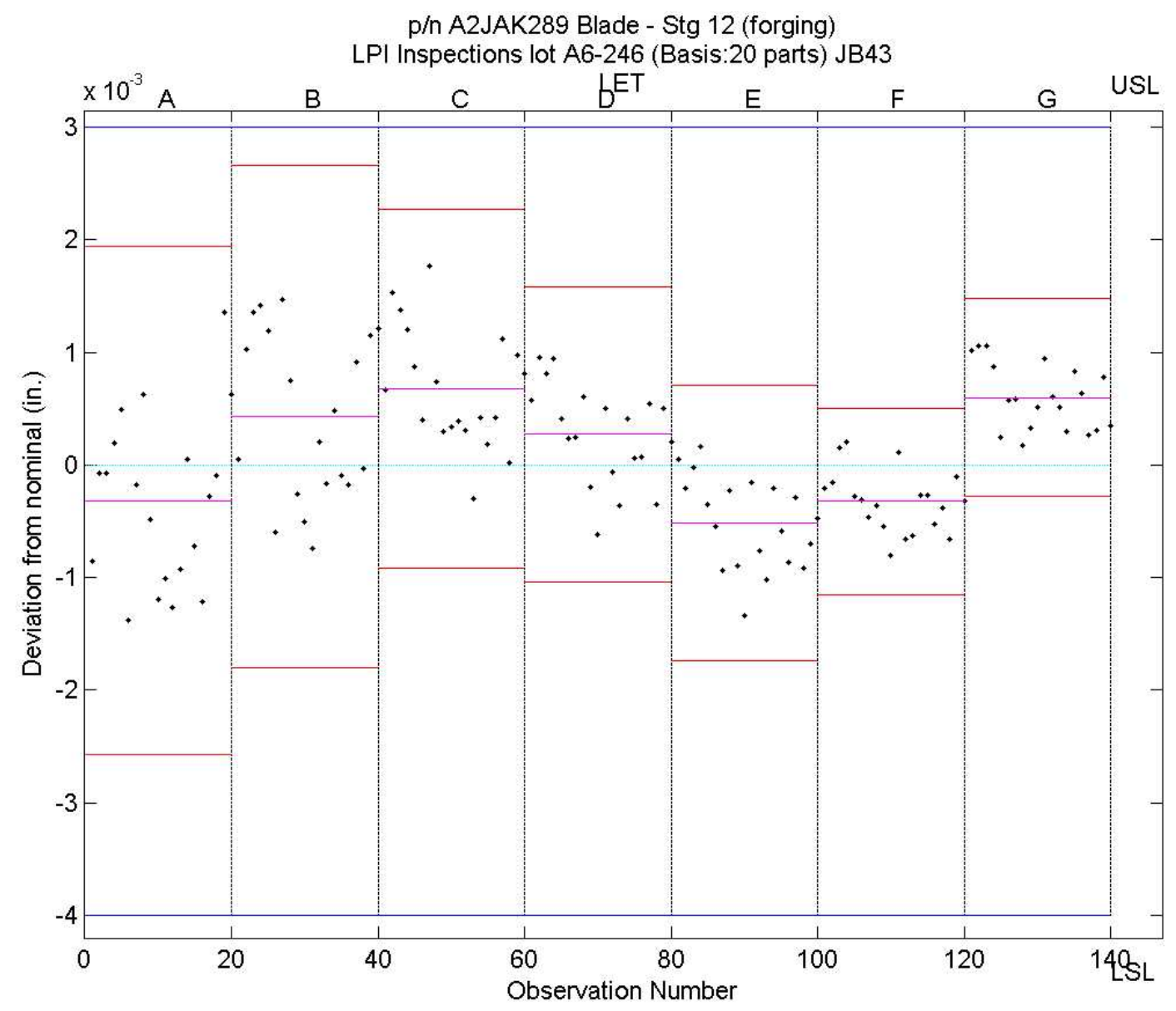

Figure 5-19: Leading Edge Thickness Section A-G 
As shown in Figure 5-20, the LET (Leading Edge Thickness) final calculations are done using the Equation 7. The UCL and LCL were calculated using equation 16. The USL and LSL are identical for all the sections. All other plot features are similar to other plots.

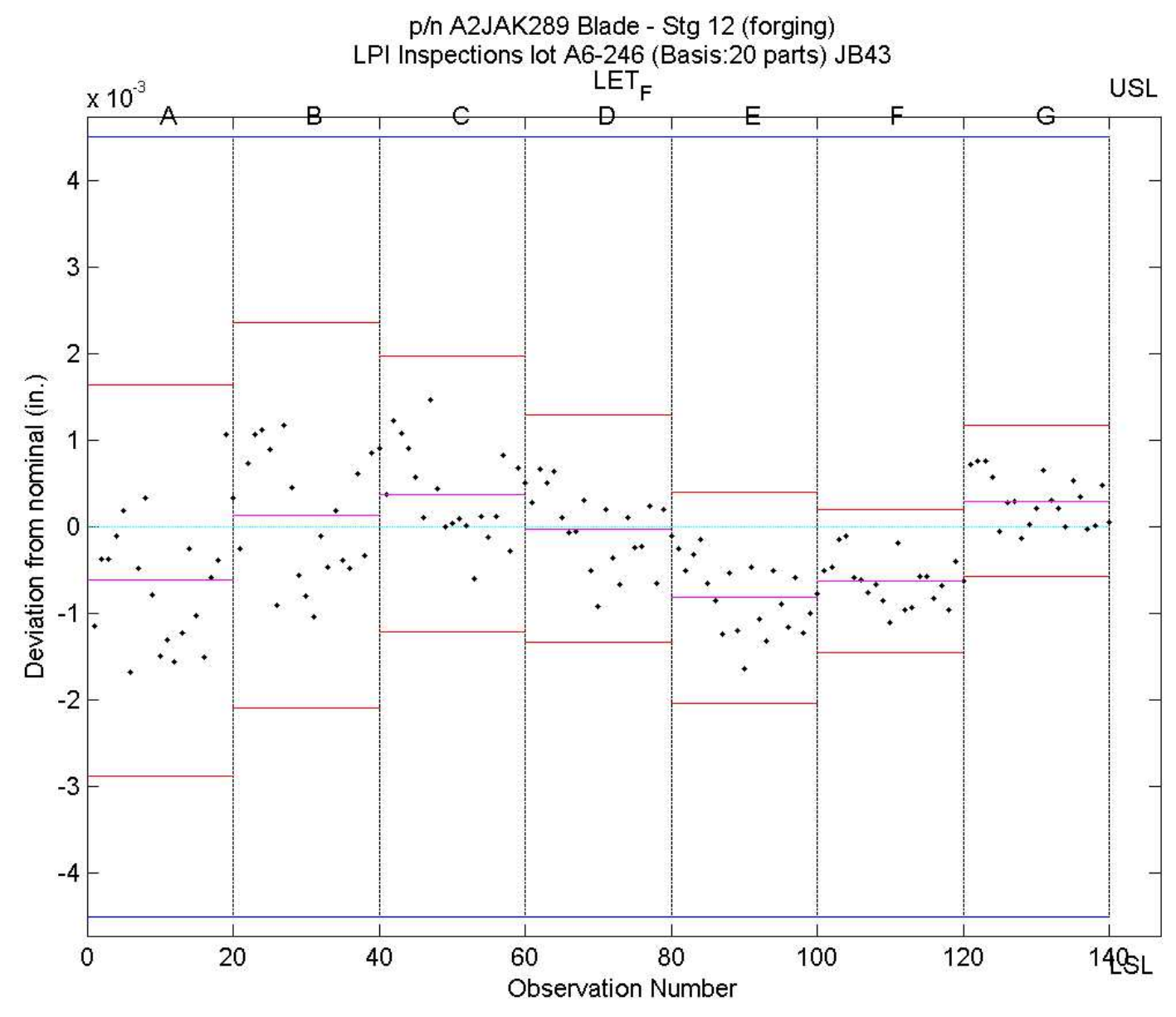

Figure 5-20: Leading Edge Thickness Final Section A-G 
As shown in Figure 5-21, the TET (Trailing Edge Thickness) is plotted similar to LET. The UCL and LCL were calculated using Equation 16. The USL and LSL are identical for all sections.

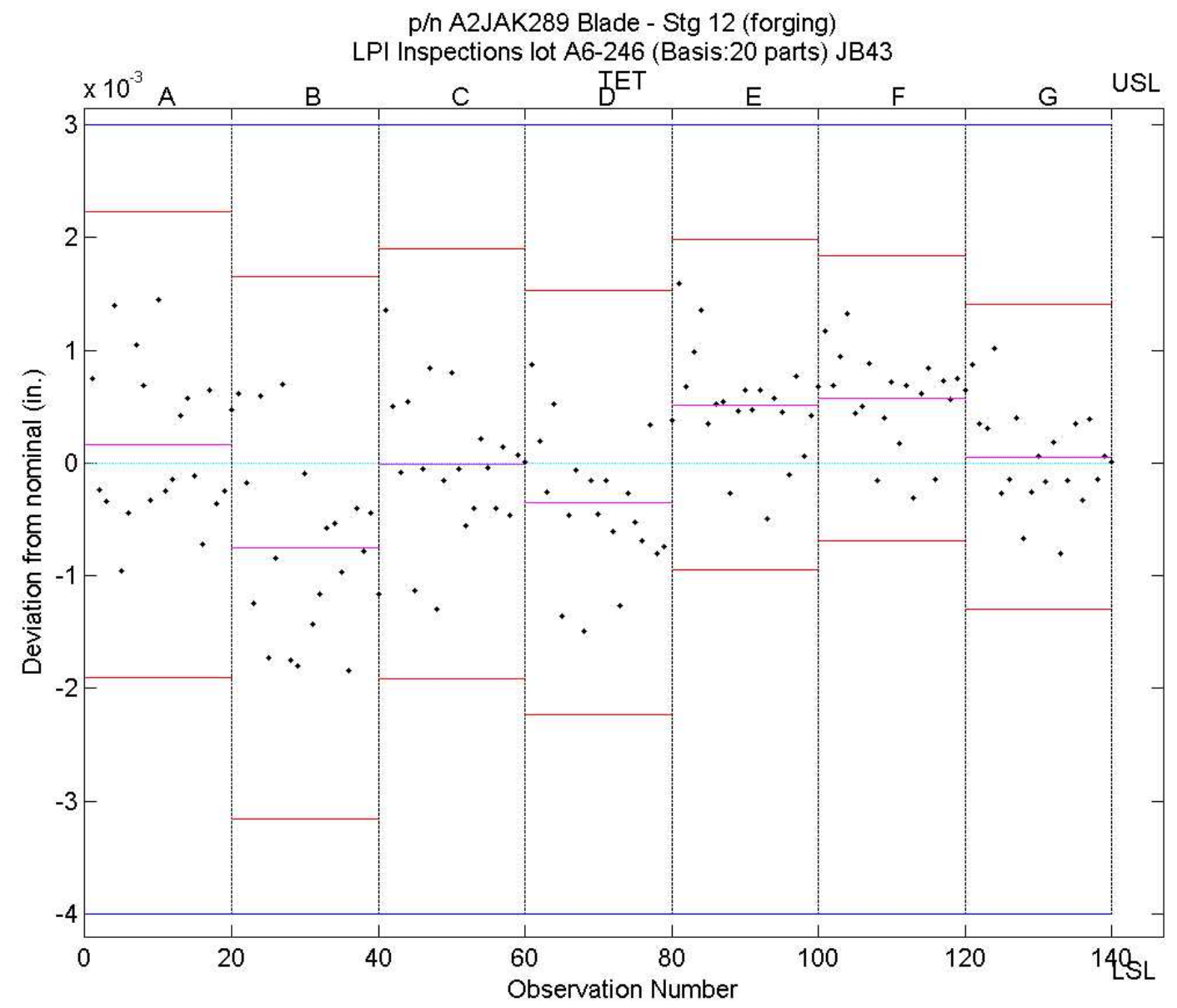

Figure 5-21: Trailing Edge Thickness Section A-G 
As shown in Figure 5-22, the TET (Leading Edge Thickness) final calculations are done using the Equation 8. The UCL and LCL were calculated using equation 16. The USL and LSL are identical for all sections. All other plot features are identical to the other plots.

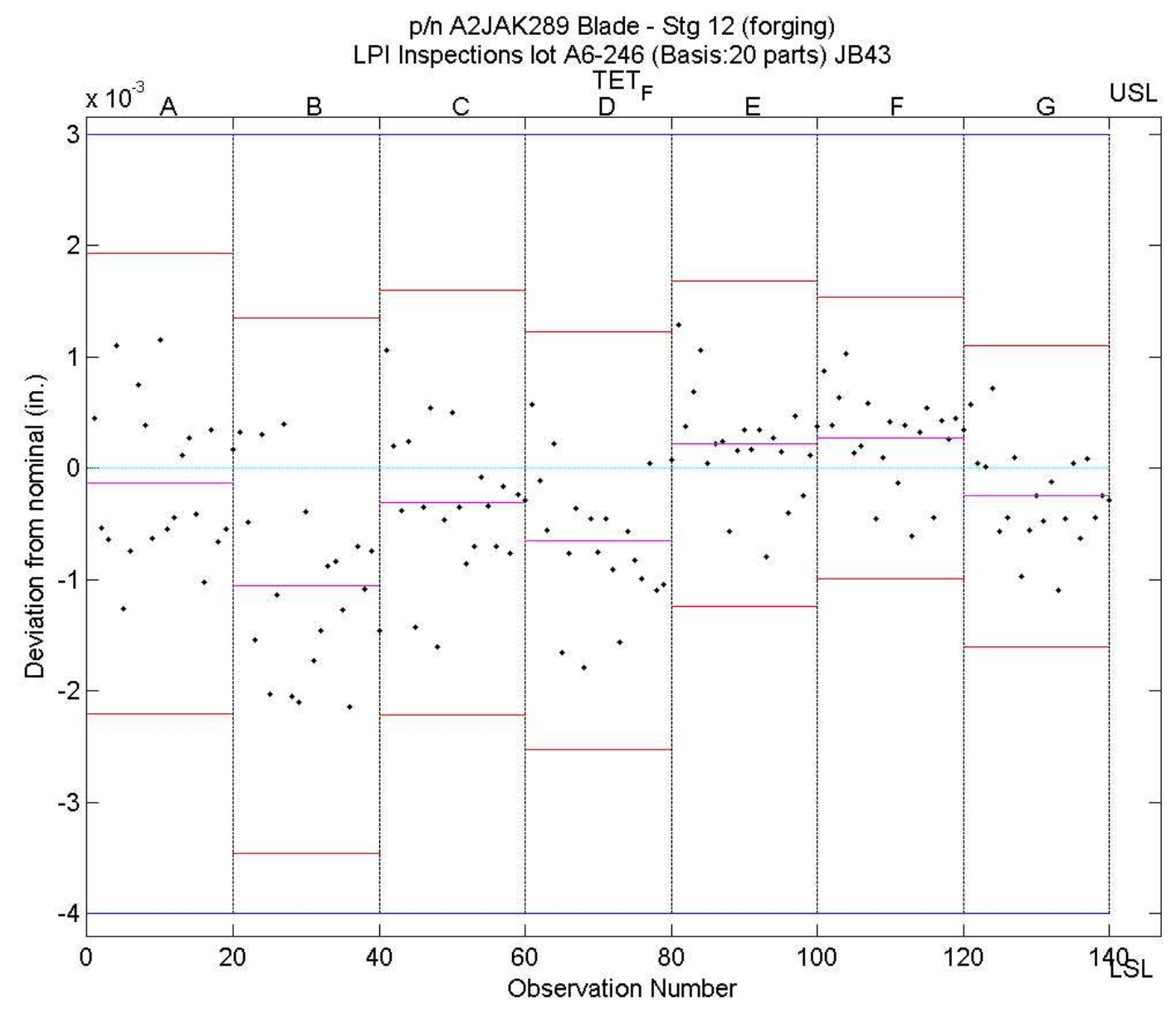

Figure 5-22: Trailing Edge Thickness Final Section A-G 
As shown in Figure 5-23, the MXT (Maximum Edge Thickness) is plotted similar to LET and TET. The UCL and LCL were calculated using Equation 16. The USL and LSL are identical for all sections. All other plot features are identical to the other plots.

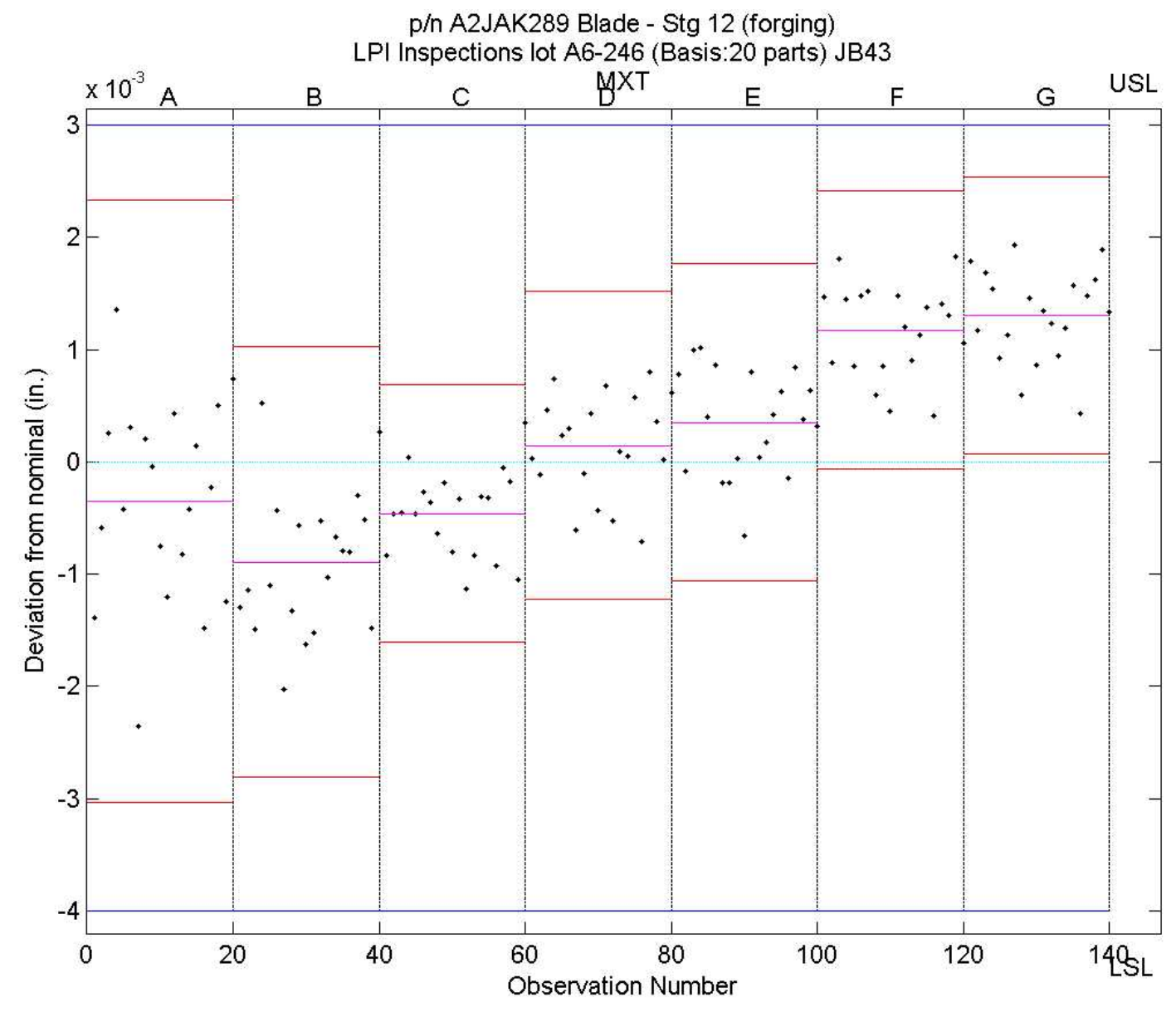

Figure 5-23: Maximum Thickness Section A-G 
As shown in Figure 5-24, the MXT (Leading Edge Thickness) final calculations are done using the Equation 9. The UCL and LCL were calculated using equation 16. The USL and LSL are identical for all sections. All other plot features identical to the other plots.

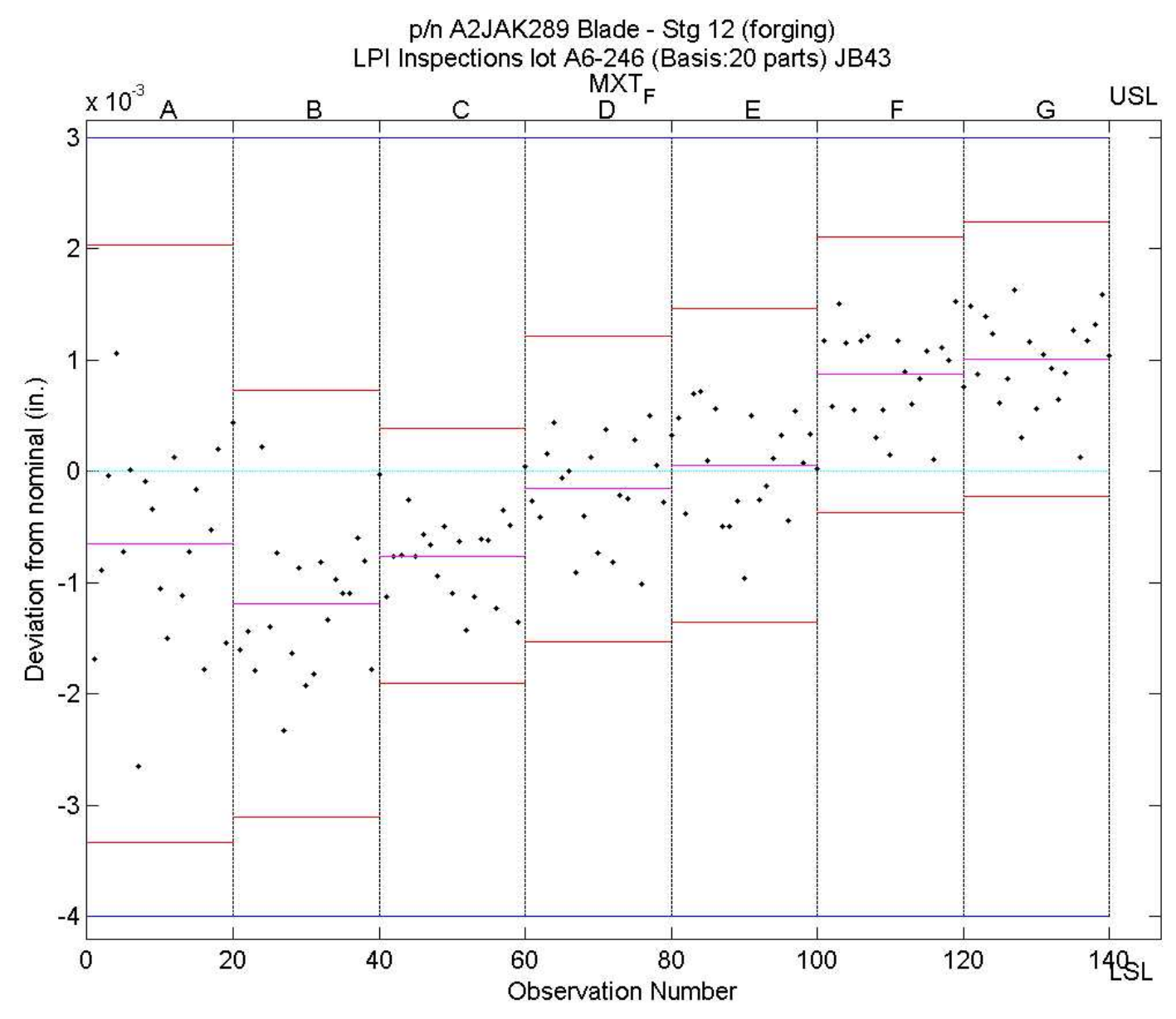

Figure 5-24: Maximum Thickness Final Section A-G 
As shown in Figure 5-25, the post-peen N-angle was calculated using Equations 10 and 14. The USL and LSL values are identical after final processing for each section. The $\mathrm{N}$-angle target was calculated using the maximizer999 algorithm. A box plot is used which still shows the UCL and LCL using the red lines for each sections.

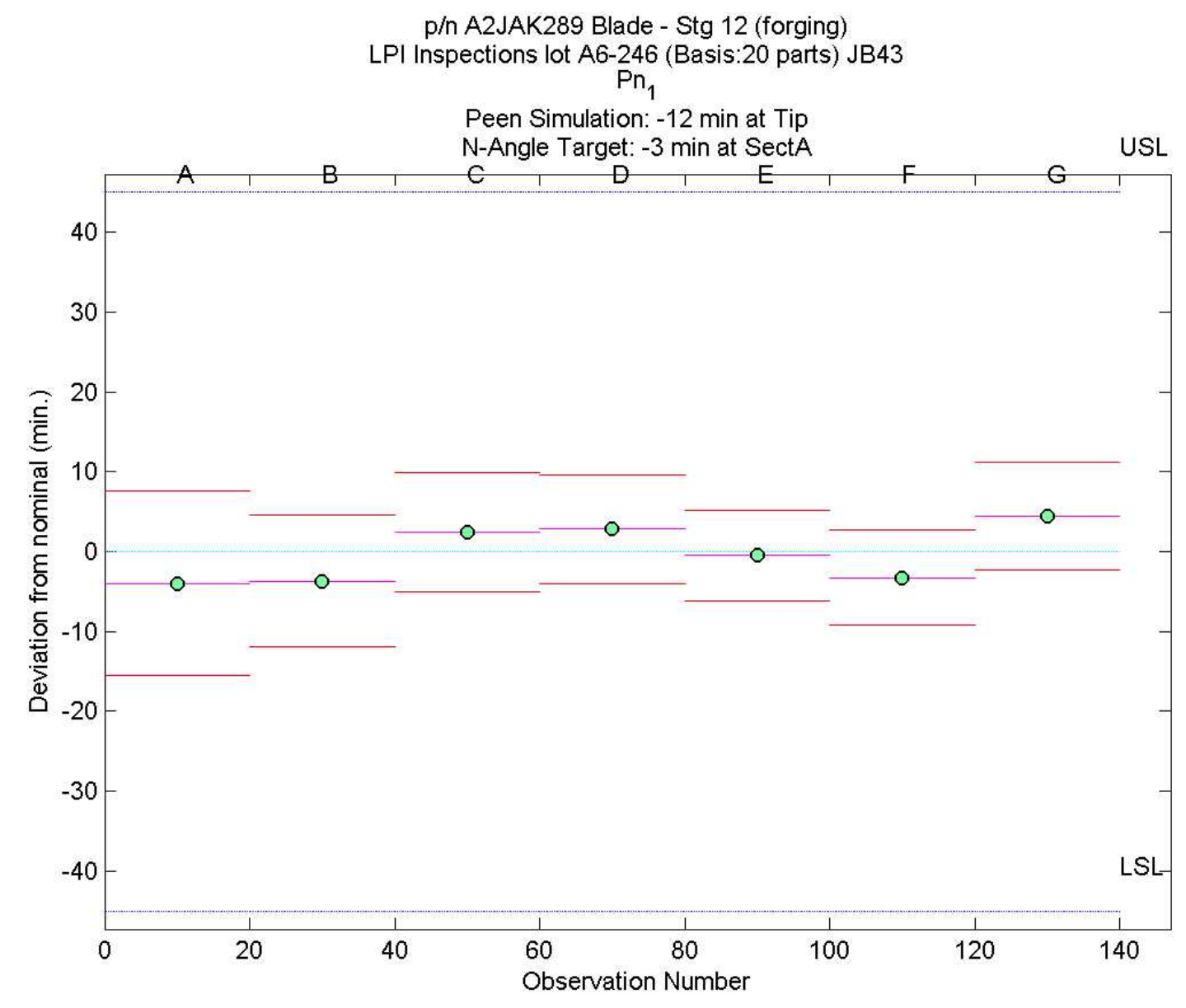

Figure 5-25: N-angle (post-peen) Section A-G with N-angle target offset 
As shown in Figure 5-26, the post-peen LEA was calculated using Equations 11 and 14. The USL and LSL values are identical after final processing for each section. The $\mathrm{N}$-angle target was calculated using the maximizer999 algorithm. A box plot is used which still shows the UCL and LCL using the red lines for each section.

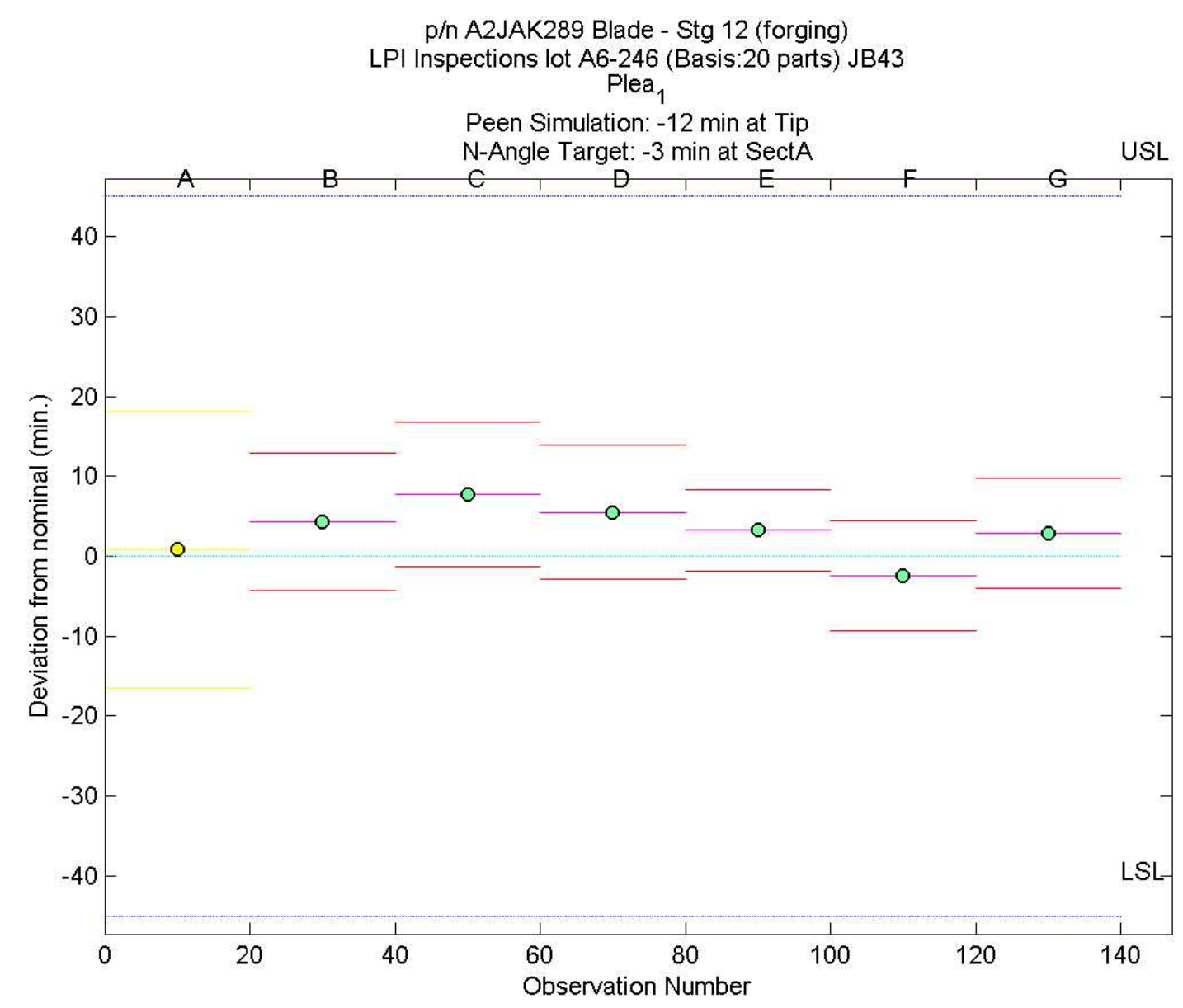

Figure 5-26: Leading Edge Angle (post-peen) Section A-G with N-angle target 
As shown in Figure 5-27, the post-peen TEA was calculated using Equations 12 and 14. The USL and LSL values are identical after final processing for each section. The $\mathrm{N}$-angle target is calculated using the maximizer999 algorithm. A box plot is used which still shows the UCL and LCL using the red lines for each sections.

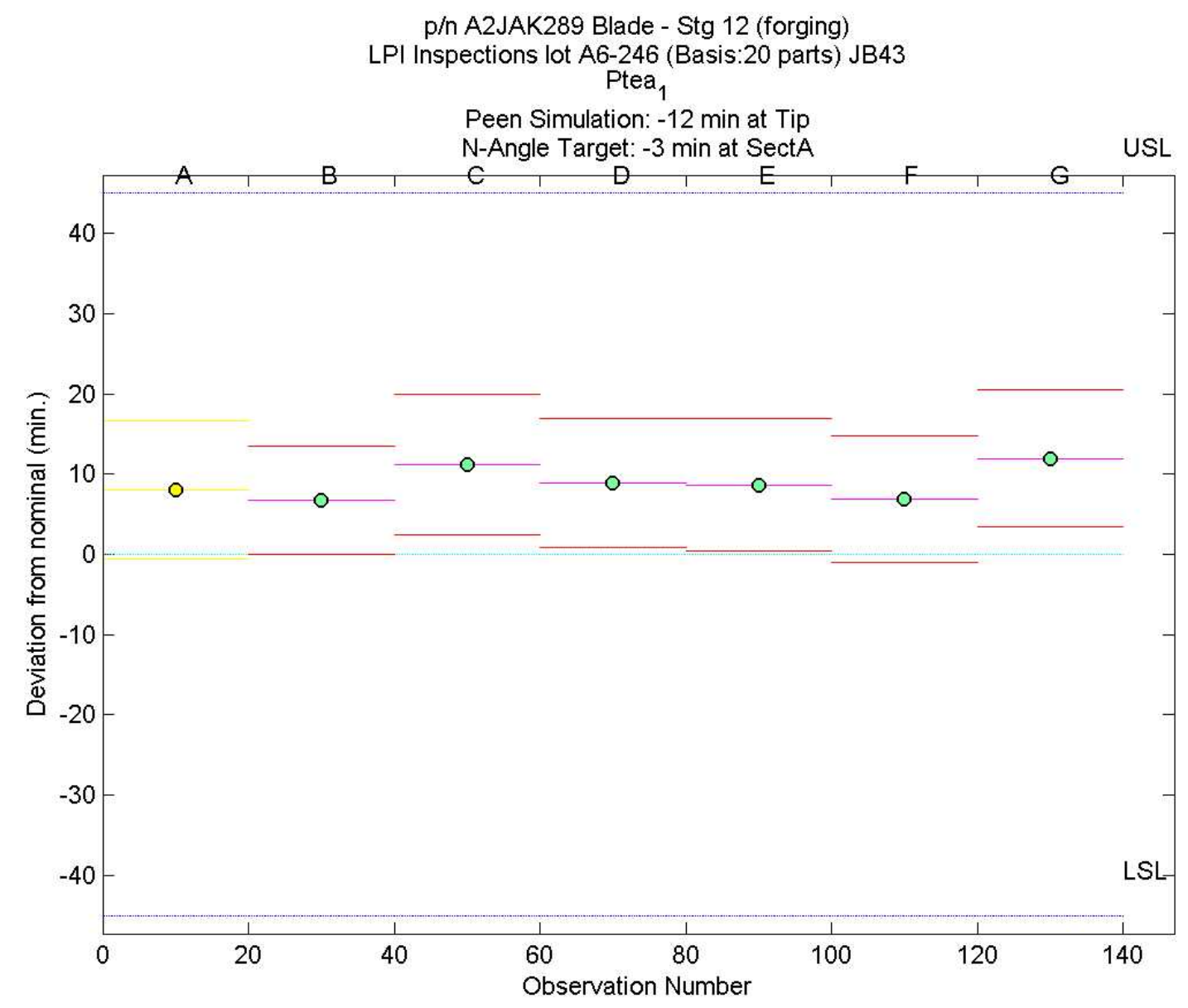

Figure 5-27: Trailing Edge Angle (post-peen) Section A-G with N-angle target 
As shown in Figure 5-28, the Adjacent Chord was calculated using Equation 1 similar to DTPX, DTPY and DTPN, the deviation difference for a given section and its immediate adjacent sections are plotted. The USL and LSL are identical for each section calculations. The UCL and LCL were calculated using Equation 16.

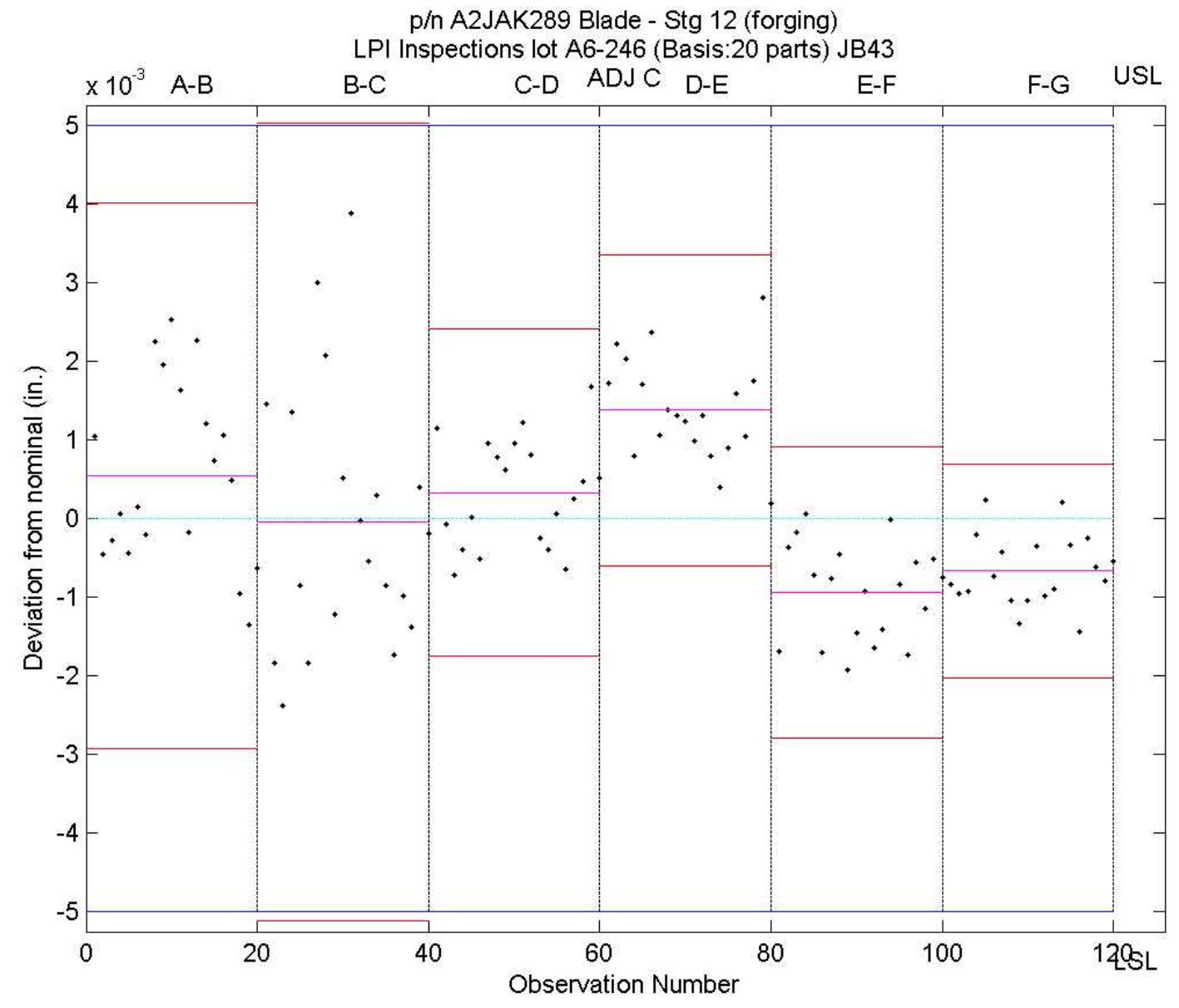

Figure 5-28: Adjacent Chord 
As shown in Figure 5-29, the Adjacent MXT was calculated using equation 1 similar to DTPX, DTPY and DTPN, the deviation difference for a given section and its immediate adjacent sections are plotted. The USL and LSL are identical for each section calculations. The UCL and LCL were calculated using Equation 16.

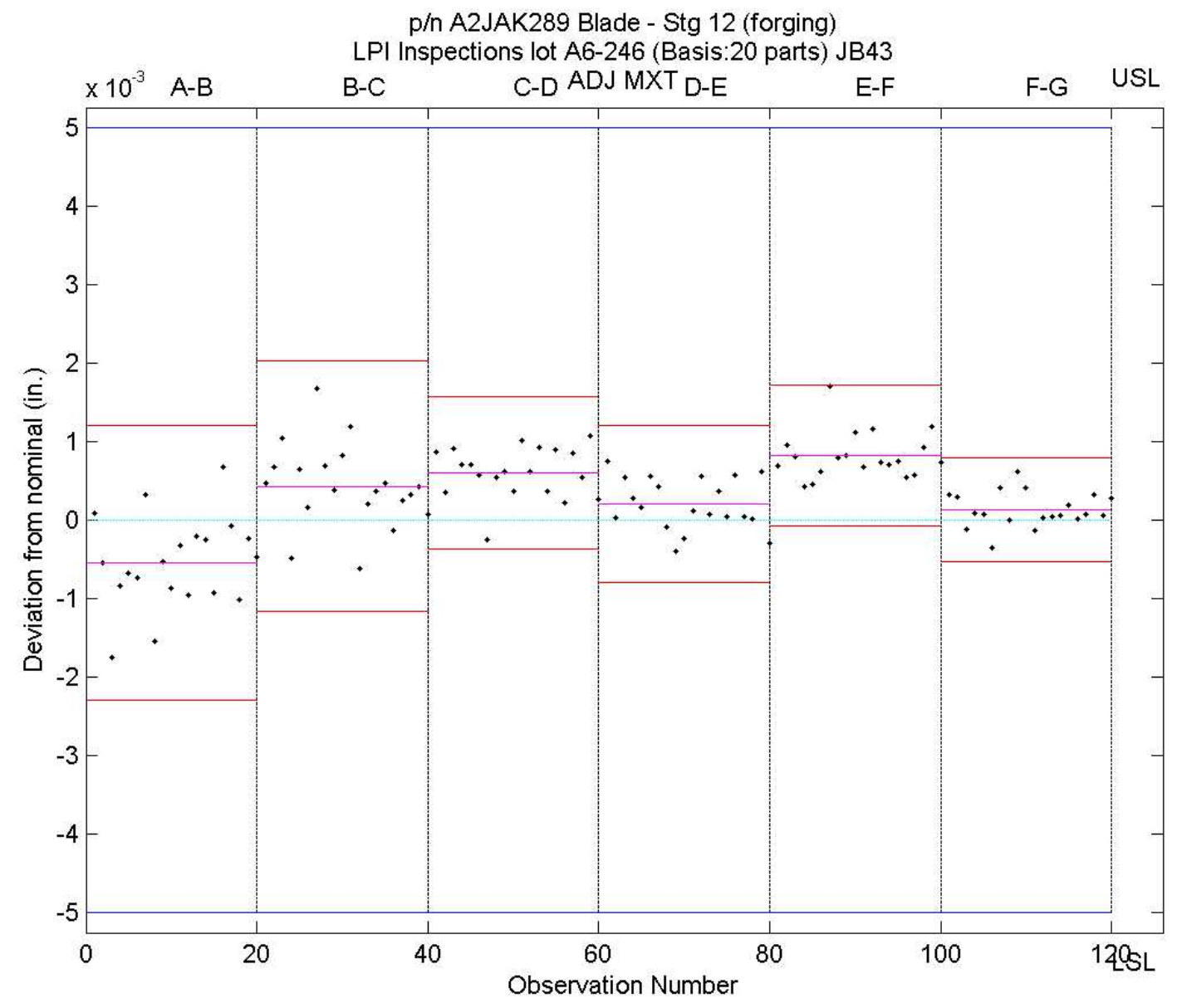

Figure 5-29: Adjacent MXT 
As shown in Figure 5-30, the platform values were plotted for each section of the platform. Since this is not an airfoil feature, it is only plotted on a scatter lot. The USL and LSL are identical for all sections.

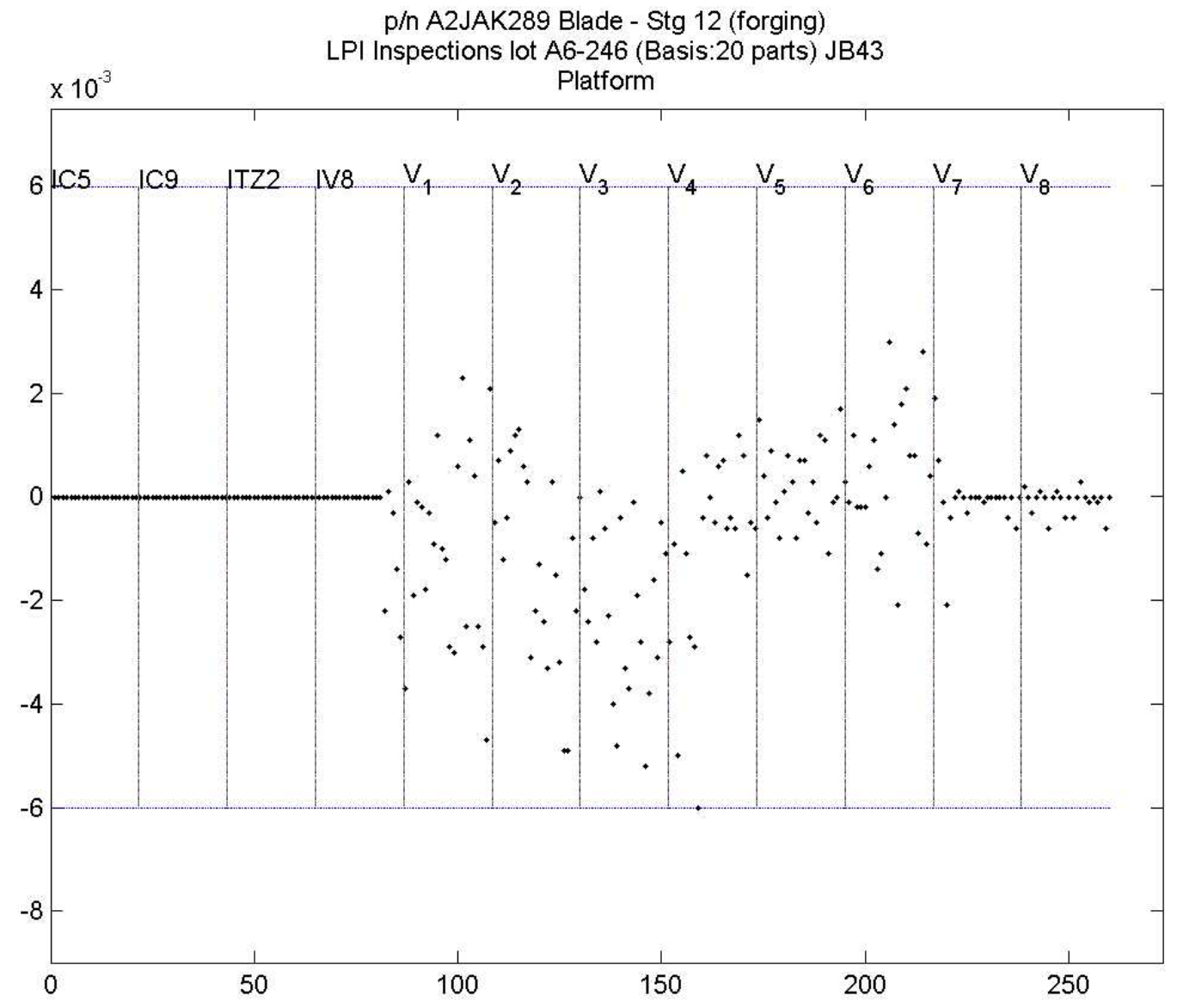

Figure 5-30: Platform deviation 
As shown in Figure 5-31, the fillet values were plotted similar to platform. This profile is not tied to any datum as it is a free form profile. The fillet values are taken on the convex side of the airfoil only, hence the section CV3 to CV5. It is plotted using a scatter plot. USL is same for all sections and it is one sided plot.

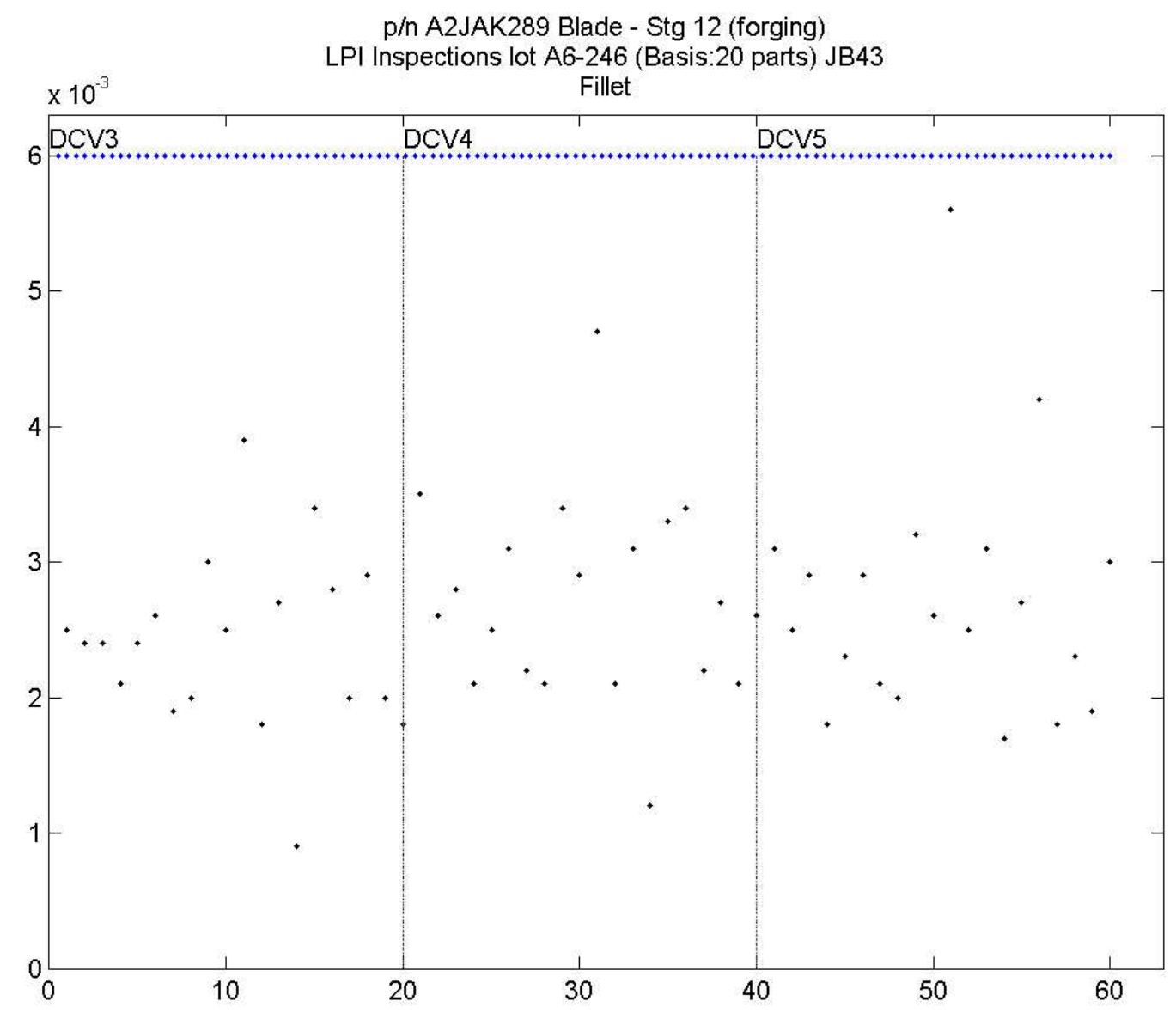

Figure 5-31: Fillet Sections CV3, CV4 and CV5 


\subsection{Data Analysis and Interpretation}

The output data from the software tool is a spreadsheet with all raw data and final calculations for all compressor blade features. The $C_{p}$ and $C_{p k}$ values (see Table 5-8) for the established features [XXX, YYY, C, N, LEA, TEA, LET, TET, and MXT] are reviewed to understand whether the forging process was in control by looking at $\mathrm{Cp}$ and the targeting of the data using $\mathrm{C}_{\mathrm{pk}}$. Typically in a manufacturing process it is common practice to aim to have $C_{p}$ and $C_{p k}$ greater than 1.33 (4-sigma), but in general $C_{p}$ and $\mathrm{C}_{\mathrm{pk}}$ values of less than 1.0 (3-sigma) are considered bad and anything greater than 1.0 (3-sigma) as good. The $\mathrm{C}_{\mathrm{pk}}$ (see Table 5-9) after final processing gives you a clear picture of how the lot is going to behave after all processing. This is a great way to focus your inspection efforts on features with bad $\mathrm{C}_{\mathrm{p}}$ and $\mathrm{C}_{\mathrm{pk}}$ and specifically their respective sections that need to be inspected to have a safety net, in case there is fallout. It is a requirement to inspect a minimum of three sections even if every feature and their corresponding sections of the lot have relatively good $\mathrm{Cp}$ and $\mathrm{Cpk}$ values. This way minimum inspection requirement is met to have enough confidence within our manufacturing process.

\section{Disposition}

Based on the results provided by the tool an engineer dispositions whether the lot is accepted or rejected. If the lot is accepted an IMS [Inspection Method Sheet] is created that has the ideal N-angle target offset and minimum sections that are needed to be inspected from the In-process stage to final stage, as shown in Figure 5-32. 


$\begin{array}{lll}\text { INSPECTION } & \text { LPI Corporation } & \text { IMS-4259 Rev N/C } \\ \text { METHOD } & & \text { November } 5,2007 \\ \text { SHEET } & & \text { Page } 1 \text { of } 1 \text { nMs.429. Doc }\end{array}$

Subject: REDUCED SECTION INSPECTION

Reduced section inspection is permitted with written authorization using the Reduced Section Inspection Authorization form below when this IMS is specified in the quality plan or IMS. The completed form shall remain on file. This authorization is limited to the lot number shown.

\section{REDUCED SECTION INSPECTION AUTHORIZATION FORM}

\begin{tabular}{|l|l|}
\hline P/N: & JAK289 \\
\hline Lot no: & A6-246 \\
\hline Lot Qty: & 120 Parts \\
\hline Inspection Basiz: & 20 Parts \\
\hline
\end{tabular}

\begin{tabular}{|c|}
\hline $\begin{array}{c}\text { SECTIONS TO } \\
\text { INSPECT AT }\end{array}$ \\
\hline B \\
\hline D \\
\hline G \\
\hline \\
\hline \\
\hline \\
\hline
\end{tabular}

Comments:

At root installation, Target $\mathrm{N}$-angle as follows:

\begin{tabular}{|c|c|}
\hline Section & N Target (degrees) \\
\hline B & -0.05 \\
\hline D & $0.10(\mathrm{ref})$ \\
\hline G & $0.25(\mathrm{ref})$ \\
\hline
\end{tabular}

Note: The targets at the outer sections are products of the targeting at the root section and the twist distribution measured in the forging.

Authorized by:

\begin{tabular}{|c|c|}
\hline Dejoet Engent & Den: \\
\hline Dertes, ABU Enginething & Dets \\
\hline ABt Culing & $D=:$ \\
\hline
\end{tabular}

Figure 5-32: IMS sheet for N-Angle target and CMM reduced section inspection 


\subsection{Software Validation}

The processing effects on all compressor blade features that were modeled are compared to actual data to validate the effects; this process has taken much iteration to fine tune the models. Certain caution is used while finalizing the models, as we took a conservative approach towards calculations of feature variations. As with any model, continuous studies have to be carried out to understand the process shifts and revalidate the calculations to accommodate any process shifts due to introducing new machines, complete new approach to machine setups etc.

One such validation to a compressor blade is shown below. The forging data from several heat codes was inspected, and feature variations were calculated using this software tool, and all the features are inspected at final CMM inspection to validate the tool and its feature variation calculations. In the final CMM inspection data, certain non-conforming parts were scrapped due to operator mishandling and visual rejections. The Table 5-4 below shows the estimated final values for each main feature calculated by the software tool, while Table 5-5 shows the actual final CMM inspection values, and Table 5-6 shows the difference between the calculated and actual values. Based on the data within the tables it can be concluded that the software tool has accurately calculated the airfoil feature variations to within the CMM inspection capability tolerances.

The cost saving shown in Table 5-13, shows the calculations based on hourly shop floor rate of $\$ 96.71$ commonly used in savings calculations. The average number of airfoil sections used is 8 , this number varied from small (6 sections) to large compressor blades (13 sections). Total number of blades used is 50 per lot and 100 lots per month. The airfoil sections are CMM inspected twice, once at the In-process stage at root installation and again at final CMM stage after all the processing has been completed. Average inspection time per section is around 3 minutes using a scanning head probe, and this varies 
with the size of the blade as well. Based on the calculations, an estimated $\$ 1,160,520.00$ is saved annually after reducing the number of airfoil sections by implementing the software.

Table 5-10: Calculated feature output from the software tool

\begin{tabular}{|c|c|c|c|c|c|c|c|c|c|c|c|}
\hline P/N & UNITS & SECT & XXX & YYY & C & N & LEA & TEA & LET & TET & MXT \\
\hline FINAL & in/min & A & -0.0032 & 0.0016 & -0.0016 & -4.0 & 0.8 & 8.0 & -0.0006 & -0.0001 & -0.0006 \\
\hline FINAL & in/min & B & -0.0034 & 0.0026 & -0.0010 & -3.7 & 4.4 & 6.8 & 0.0001 & -0.0011 & -0.0012 \\
\hline FINAL & in/min & C & -0.0036 & 0.0040 & -0.0013 & 2.4 & 7.8 & 11.1 & 0.0004 & -0.0003 & -0.0008 \\
\hline FINAL & in/min & D & -0.0032 & 0.0047 & -0.0011 & 2.8 & 5.5 & 8.8 & 0.0000 & -0.0007 & -0.0002 \\
\hline FINAL & in/min & E & -0.0038 & 0.0059 & 0.0001 & -0.5 & 3.2 & 8.6 & -0.0008 & 0.0002 & 0.0000 \\
\hline FINAL & in/min & F & -0.0038 & 0.0054 & -0.0011 & -2.8 & -2.3 & 7.2 & -0.0006 & 0.0003 & 0.0009 \\
\hline FINAL & in/min & G & -0.0040 & 0.0051 & -0.0020 & 4.5 & 2.9 & 12.0 & 0.0003 & -0.0002 & 0.0010 \\
\hline
\end{tabular}

Table 5-11: Actual feature output of manufactured lot (CMM Final Inspection)

\begin{tabular}{|c|c|c|c|c|c|c|c|c|c|c|c|}
\hline P/N & UNITS & SECT & XXX & YYY & C & N & LEA & TEA & LET & TET & MXT \\
\hline FINAL & in/min & A & -0.0027 & 0.0090 & -0.0013 & -5.3 & 1.7 & 6.3 & -0.0008 & -0.0003 & -0.0009 \\
\hline FINAL & in/min & B & -0.0026 & 0.0015 & -0.0011 & -4.2 & 4.0 & 7.1 & -0.0002 & -0.0013 & -0.0014 \\
\hline FINAL & in/min & C & -0.0029 & 0.0032 & -0.0011 & 3.9 & 5.9 & 12.9 & 0.0003 & -0.0005 & -0.0010 \\
\hline FINAL & in/min & D & -0.0033 & 0.0039 & -0.0011 & 4.1 & 4.3 & 10.1 & -0.0002 & -0.0008 & -0.0004 \\
\hline FINAL & in/min & E & -0.0031 & 0.0053 & -0.0001 & 0.9 & 2.4 & 6.9 & -0.0010 & -0.0001 & -0.0002 \\
\hline FINAL & in/min & F & -0.0039 & 0.0059 & -0.0008 & -1.9 & 3.4 & 7.9 & -0.0008 & 0.0000 & 0.0006 \\
\hline FINAL & in/min & G & -0.0043 & 0.0048 & -0.0021 & 5.3 & 3.9 & 10.5 & 0.0000 & -0.0004 & 0.0007 \\
\hline
\end{tabular}

Table 5-12: Difference between calculated and actual feature output

\begin{tabular}{|c|c|c|c|c|c|c|c|c|c|c|c|}
\hline P/N & UNITS & SECT & XXX & YYY & C & N & LEA & TEA & LET & TET & MXT \\
\hline DIFF & in/min & A & 0.0005 & 0.0074 & 0.0003 & -1.3 & 0.9 & -1.6 & -0.0002 & -0.0002 & -0.0003 \\
\hline DIFF & in/min & B & 0.0008 & -0.0011 & -0.0001 & -0.5 & -0.4 & 0.4 & -0.0003 & -0.0003 & -0.0002 \\
\hline DIFF & in/min & C & 0.0007 & -0.0008 & 0.0002 & 1.5 & -1.9 & 1.8 & -0.0001 & -0.0002 & -0.0002 \\
\hline DIFF & in/min & D & -0.0001 & -0.0008 & 0.0000 & 1.3 & -1.2 & 1.3 & -0.0002 & -0.0001 & -0.0003 \\
\hline DIFF & in/min & E & 0.0007 & -0.0006 & -0.0002 & 1.4 & -0.8 & -1.7 & -0.0002 & -0.0003 & -0.0002 \\
\hline DIFF & in/min & F & -0.0001 & 0.0005 & 0.0003 & 0.9 & 5.7 & 0.7 & -0.0002 & -0.0003 & -0.0003 \\
\hline DIFF & in/min & G & -0.0003 & -0.0004 & -0.0002 & 0.8 & 1.0 & -1.5 & -0.0003 & -0.0002 & -0.0003 \\
\hline
\end{tabular}


Table 5-13: Cost saving before and after tool implementation

\begin{tabular}{|c|c|c|c|c|}
\hline Items & \multicolumn{2}{|r|}{ Before } & \multicolumn{2}{|r|}{ After } \\
\hline Average no of sections per blade & & 8 & & 4 \\
\hline Blades/Lot & & 50 & & 50 \\
\hline Lots/Month & & 100 & & 100 \\
\hline Blades/Year & & 60000 & & 60000 \\
\hline CMM Inspection count (In-Process, Final) & & 2 & & 2 \\
\hline Average Inspection Time (minutes/section) & & 3 & & 3 \\
\hline Average Inspection Time (minutes/blade) & & 24 & & 12 \\
\hline Shop Floor Rate $(\$ / \mathrm{hr})$ & $\$$ & 96.71 & $\$$ & 96.71 \\
\hline CMM Inspection Cost (\$/blade) & $\$$ & 38.68 & $\$$ & 19.34 \\
\hline Cost per lot & $\$$ & $3,868.40$ & $\$$ & $1,934.20$ \\
\hline Cost per month & $\$$ & $386,840.00$ & $\$$ & $193,420.00$ \\
\hline Cost per year & $\$$ & $2,321,040.00$ & $\$$ & $1,160,520.00$ \\
\hline Cost savings per year (approx) & & & & $1,160,520.00$ \\
\hline
\end{tabular}




\section{CHAPTER 6. CONCLUSION AND FUTURE WORK}

\subsection{Conclusion}

In conclusion the objective of this thesis is to develop and implement a software tool to calculate the airfoil feature variations throughout the manufacturing process. The author did not come across any literature where such a tool was presented or described, at least not on open literature. The tool was developed mainly to help reduce the number of airfoil sections that are being inspected by using the process control data $\left(\mathrm{C}_{\mathrm{p}}, \mathrm{C}_{\mathrm{pk}}\right)$. The reduced section inspection was justified based on validation results for each stage compressor blade's forging airfoil inspection data, where each of the feature values that are estimated by the tool after all manufacturing process is compared to the actual process data. Only after the data is validated, by making sure that the tool is predicting the feature variations per processing models, the tool is approved and implemented for that stage blade. The reduced sections are chosen based on the $\mathrm{C}_{\mathrm{p}}$ and $\mathrm{C}_{\mathrm{pk}}$ values after all processing; the criteria suggested to choose a particular section to be inspected is if for that section the airfoil feature $\mathrm{C}_{\mathrm{p}}$ and $\mathrm{C}_{\mathrm{pk}}$ values are $<1$ (3-sigma), and if all the sections have values greater than 1 , then only a minimum of three sections are required to be inspected for the blade, usually bottom, middle and top section of the blade. This ensures that quality of the airfoil is not compromised as those three sections are inspected for all compressor blades in that lot.

The $\mathrm{N}$-angle target is a substantial aid to the grind operators as they target at an optimal $\mathrm{N}$-angle offset suggested as opposed to targeting at nominal. This eliminated all the fallouts after final CMM inspection due to N-angle, LEA and TEA non-conformances. 
And last but the least, the team is now able to disposition the forging lot as accept or reject based on the information provided. This saved a lot of scrapped parts that would have otherwise been processed and provided huge cost savings for the company.

Since the implementation of the tool, the business unit has saved approximately $\$ \$ 1,160,520.00$ per year; numbers are calculated based on $\$ 96.71 / \mathrm{hr}$ shop floor compensation rate. This dollar amount is based on average 8 section blade and the numbers are also calculated using a conservative estimate. The biggest impact is the dollars we have saved for the business, also potentially eliminating the bottleneck operation that was the CMM inspection.

\subsection{Future Work}

Future enhancements to the software tool might help generic audiences with the tool usage, for example writing the tool as a standalone executable will eliminate the need of having the parent software installed on the computer. Improved graphical user interface would help the users get a progress bar to understand what the status of the tool is. Automation of the current manual data crunching of the CMM inspection data (Input data) and programming the tool in a way the data output from the CMM can be directly used as the input to the tool would further enhance this tool. Another farfetched idea is to use the $\mathrm{N}$-angle offset target as a live tool where the data can be adjusted real time after each CMM inspected part that feeds into the tool real time and gives an automatic $\mathrm{N}$-angle target for the next part improving the yield of the lot. Lastly use similar methodologies towards root inspection and help reduce the number of root features inspected, this would significantly help in cutting down the CMM inspection time while providing cost savings to the business. 


\section{REFERENCES}

[1] E. Torenbeek, H. W. (2002). Flight Physics: Essentials of Aeronautical Disciplines and Technology, with Historical Notes. London, NY: Springer Dordrecht Heidelberg.

[2] Allan, R. A. (2001). A History of the Personal Computer: The People and the Technology. London, Ontario, Canada: Allan Publishing.

[3] O'Regan, G. (2008). A Brief History of Computing. New York: Springer London Dordrecht Heidelberg.

[4] Hunecke, K. (2003). Jet Engines: Fundamentals of Theory, Design and Operation. Osceola, WI: Motorbooks International Publishers \& Wholesalers.

[5] Brown, R. N. (2005). Compressors: Selection And Sizing. Elsevier, Inc.

[6] http://www.wisegeek.com/. (n.d.). Retrieved from http://www.wisegeek.com/:

http://www.wisegeek.com/what-is-an-airfoil.htm

[7] Siṃha, R. (2006). Introduction To Basic Manufacturing Process \& Workshop Technology. New Delhi: New Age International (P) Ltd, Publishers.

[8] FIA: Forging Industry Association. (n.d.). https://www.forging.org/forging-facts\#1. Retrieved 2012, from http://www.forging.org: http://www.forging.org

[9] Hocken, R. J. (1995). Coordinate Measuring Machines and Systems. New York: Marcel Dekker, Inc.

[10] Boyce, M. P. (2012). Gas Turbine Engineering Handbook. Oxford: Elsevier Inc.

[11] FAA-H-8083-3A. (2004). Airplane Flying Handbook. U.S. Department of Transporation.FAA.

[12] Axial Compressor. (n.d.). Retrieved 11 2012, from wikipedia:

http://en.wikipedia.org/wiki/Axial_compressor

[13] Oates, G. C. (1985). Aerothermodynamics of Aircraft Engine Components. N.Y: American Institute of Aeronautics and Astronautics, Inc.

[14] JA650. (02/14/2002). Engineering SPecification. Airfoil Geometric Definition, Terminology, and Methods .

[15] Wiki_GE_CF6. (n.d.). Retrieved from http://en.wikipedia.org/wiki/General_Electric_CF6

[16] wiki_CFM56. (n.d.). Retrieved from http://en.wikipedia.org/wiki/CFM_International_CFM56 
[17] Hitech Alloy Products. (n.d.). Retrieved 11 2012, from www.hitechalloys.com: http://www.hitechalloys.com/hitechalloys_002.htm

[18] Grinding (abrasive cutting). (n.d.). Retrieved 05 2012, from wikipedia.org: http://en.wikipedia.org/wiki/Grinding_\%28abrasive_cutting\%29

[19] Benedict, G. F. (1987). Nontraditional Manufacturing Processes. N.Y: Marcel Dekker, Inc.

[20] Donald O. Thompson, D. E. (1997). Review of Progress in Quantitative Nondestructive Evaluation, Volume 16. N.Y: Plenum Press, New York.

[21] Fluorescent Penetrant Inspection. (n.d.). Retrieved 11 2012, from http://turbineresourcesinc.com: http://turbineresourcesinc.com/services/services.htm

[22] Fluorescent penetrant inspection materials. (n.d.). Retrieved 11 2012, from http://www.tjskl.org.cn/:

http://www.tjskl.org.cn/products/cza23159/fluorescent_penetrant_inspection_materials-pz25d74cd.html

[23] Shot Peening. (n.d.). Retrieved from www.wikipedia.org:

http://en.wikipedia.org/wiki/Shot_peening

[24] Hocken, R. (2011). Coordinate measuring machines and systems. CRC Press Inc.

[25] PC-DMIS Blade. (n.d.). Retrieved 11 2012, from www.pcdmis.com:

http://www.pcdmis.com/products/pc-dmis-blade

[26] Barbara F. Ryan, B. L. (2005). Minitab Handbook: Updated for Release 14. Canada: Thomson Learning, Inc.

[27] MATLAB: Guide by Higham, Desmond J; Higham, Nicholas J; Books24x7, Inc

[28] Defense, D. o. (1989). MIL-STD-105E. U. S. Army Armament Research, Development and Engineering Center. 


\section{APPENDIX: SOURCE CODE}

\section{Main Program:}

function forecast(str)

close all

clc

global bladecount c_loss $r$ lot sectQ

global header sect closure Pref partfile

global Naoffset Psim Ftol Ptol

disp('Please select from the following: ')

disp('1 - Full Forecast (Lots over 100 pieces)')

disp('2 - Limited Forecast (Lots 100 pieces and less)')

choice $=$ input $\left({ }^{\prime}\right.$ Please choose a number and press enter: ');

clc

disp('Please select from the following: ')

disp('1 - Automatic N-Angle Targeting')

disp('2 - Manual N-Angle Entry')

nChoice $=$ input $\left({ }^{\prime}\right.$ Please choose a number and press enter: ' $)$;

clc

\%Read in and determine size of the raw data from the Excel

Spreadsheet

[num txt raw] $=x$ lsread $(s t r$, 'Sheet1'); clear txt; \%Reading in excel file with raw data.

$\left[\begin{array}{ll}r & c\end{array}\right]=\operatorname{size}(r a w) ;$

lot $=\operatorname{raw}\{2,2\} ; \quad \%$ Sets the lot name from the raw data

partFile $=\operatorname{raw}\{2,1\} ;$ Finds what part is being estimated

\%Determine what sections are present and how many sections in total section $=\operatorname{raw}\{2,7\}$;

count $=1$;

sectQ $=[]$;

sectcount $=[]$;

for index $=3: r$

test $=\operatorname{raw}\{$ index, 7$\}$;

if strcmpi(test, section)

count $=$ count +1 ;

if index $==r$

sectQ $=[$ sectQ, section $]$;

sectCount $=[$ sectCount, count $]$;

end 


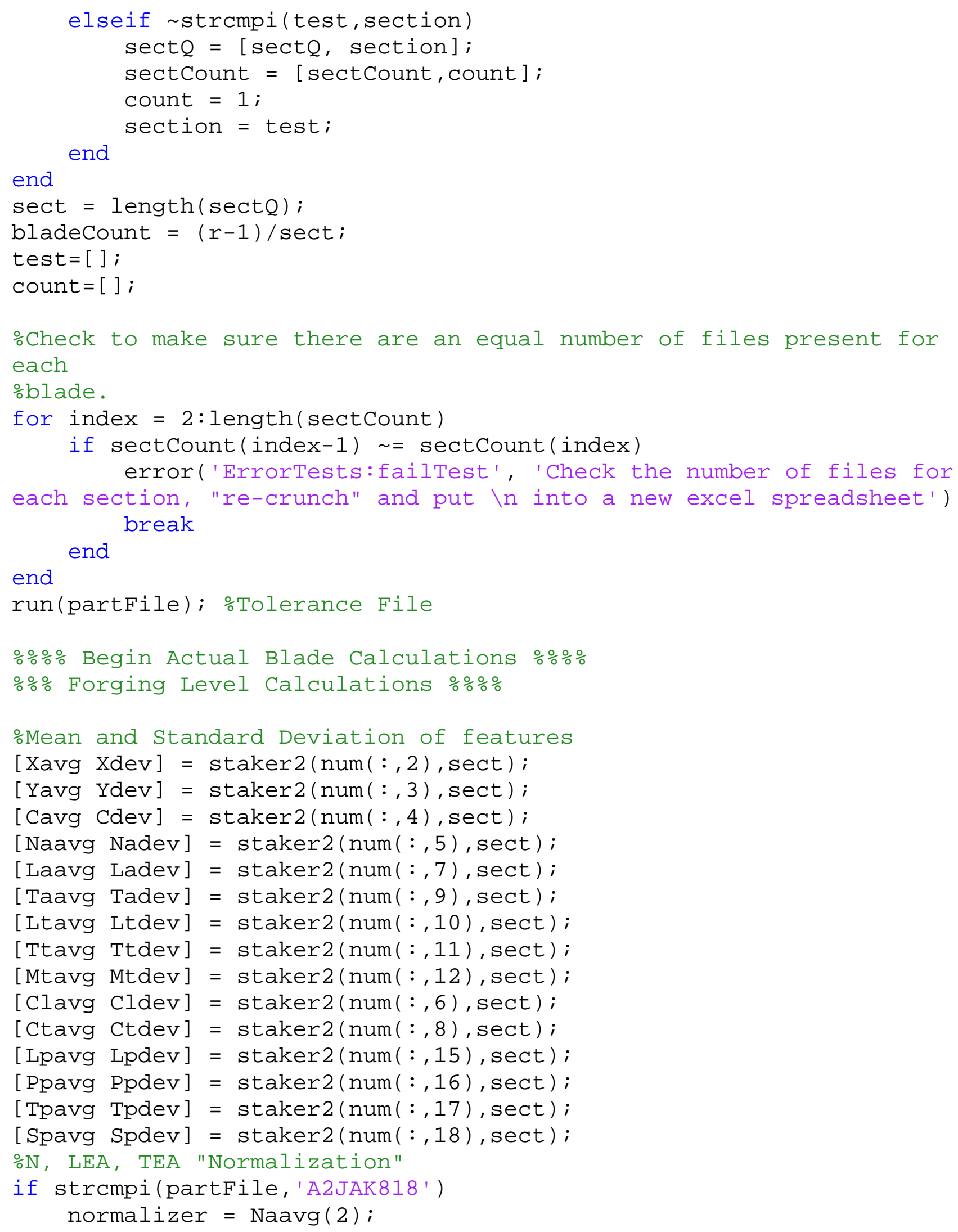




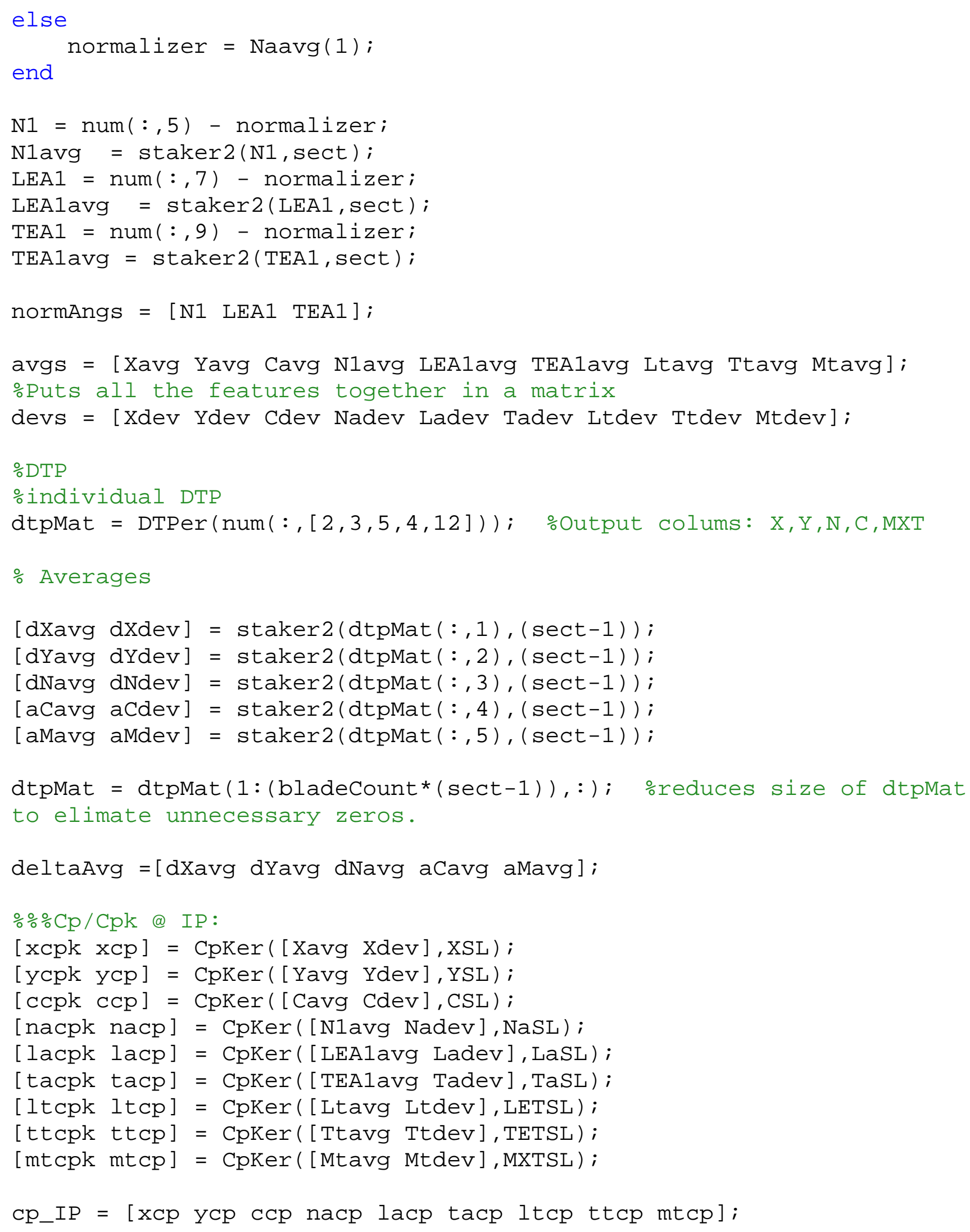


cpk_IP $=$ [xcpk ycpk ccpk nacpk lacpk tacpk ltcpk ttcpk mtcpk];

IPpc $=[$ cP_IP; $z \operatorname{zeros}(1,9) ; c$ pk_IP $]$;

$\% \%$ Estimated Final Estimates \%\%

\%Chord@Final:

[cfAvg cfDev cfMat $]=\operatorname{chordCalcs}($ num $) ;$

\%LET, TET, MXT Final Calculation:

LET $=\operatorname{num}(:, 10)$;

LET_F = LET + etch;

Ltfavg = Ltavg + etch;

TET = num $(:, 11)$;

TET_F = TET + etch;

Ttfavg = Ttavg + etch;

MXT = num $(:, 12)$;

MXT_F = MXT + etch;

Mtfavg = Mtavg + etch;

\%Peen Simulation

if nChoice $==1$

NaOffset $=\operatorname{maximizer} 599($ normAngs, $\operatorname{devs}(:, 4: 6), \mathrm{PnSL})$;

elseif nchoice $==2$

disp( ' ' )

Naoffset = input(' Enter the desired N-Angle Offset: ');

end $\operatorname{disp}($ ' ' $)$

Pn1 = peenER (N1, NaOffset $)$;

PNavg = staker $2(\mathrm{Pn} 1$, sect $)$;

Plea1 = peenER (LEA1, Na0ffset $)$;

PLavg = staker2(Plea1, sect);

Ptea1 $=$ peenER $($ TEA1, Na0ffset $)$;

PTavg = staker2 $($ Ptea1, sect $)$;

peenData $=[$ Pn1 Plea1 Ptea1 $] ;$

\%CpK@Final:

cfcpk = CpKer ([cfAvg cfDev], CFSL);

nafcpk $=$ CpKer ([PNavg Nadev], PnSL);

lafcpk = CpKer ([PLavg Ladev],PISL);

tafcpk = CpKer ([PTavg Tadev], PtSL);

ltfcpk = CpKer ([Ltfavg Ltdev], LET_FSL);

ttfcpk $=$ cpKer $([$ Ttfavg Ttdev $]$, TET_FSL $)$;

mtfcpk $=$ CpKer ([Mtfavg Mtdev], MXT_FSL); 
cpk_F $=$ [cfcpk nafcpk lafcpk tafcpk ltfcpk ttfcpk mtfcpk];

\%rite to the Excel spreadsheet:

$\%$ create the raw data spreadsheet

raw $=$ spreadsheetMaker ( raw, sectCount, avgs, devs, dtpMat, deltaAvg, ... cfMat, cfAvg, normAngs, [N1avg, LEA1avg, TEA1avg], peenData, ... [PNavg, PLavg, PTavg], [LET_F, TET_F, MXT_F] );

filename = [partFile '_' lot '_rawData.Xls'];

\%rite raw data

xlswrite(filename, raw, 'Raw Data')

\%Write IP cp/cpk data

xlswrite(filename, IPpc, 'Cp-Cpk at IP')

\%write FINAL cpk data

xlswrite(filename, cpk_F,'CPK at FINAL')

\% Plot data

\%create text for graphs

ref_onlyText = 'Information 0nly - not a product requirment';

if strcmp('A2JAK818', partFile) else

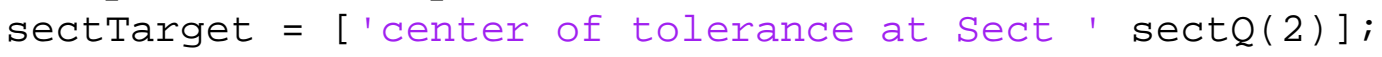

end

sectTarget $=[$ 'center of tolerance at sect ' sectQ(1)];

AngleTargetText $=[$ 'N-Angle distribution adjusted to',$\ldots$ sectTarget ];

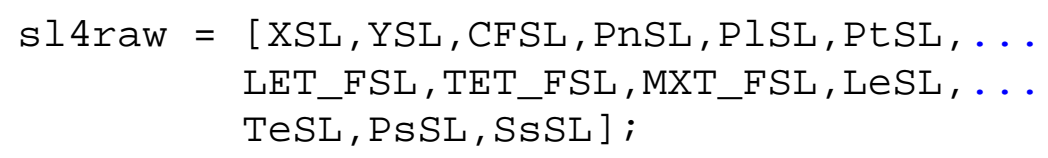

if choice $==1$

\%Plot ALL features

normalPlot(XSL, ' ' , 'XXX', num (:, 2), Xavg, Xdev)

$\%$ figure

normalPlot(DTPSL, ' ', 'DTP X', dtpMat $(:, 1), \mathrm{dXavg}, \mathrm{dXdev}$ )

$\%$ figure

normalPlot(YSL, ' ', 'YYY', num(: , 3), Yavg, Ydev)

$\%$ figure

normalPlot(DTPSL, ' ', 'DTP Y',dtpMat $(:, 2)$, dYavg, dYdev)

$\%$ figure

normalPlot (CSL, ' ', 'C' , num(: ,4), Cavg, Cdev)

$\%$ figure

normalPlot (CFSL, ' ', 'C_f', cfMat, cfAvg, cfDev, chordText)

$\%$ figure 


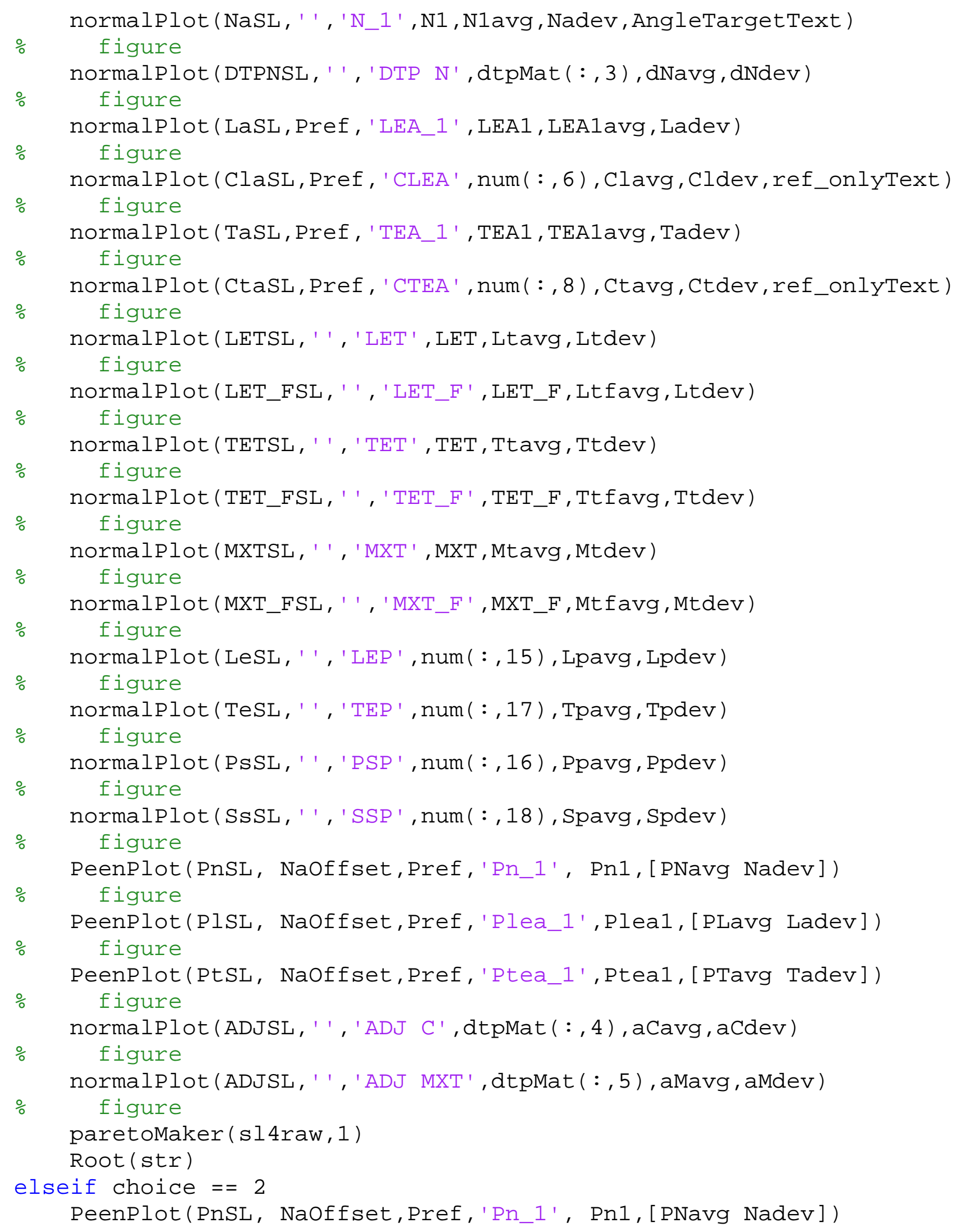




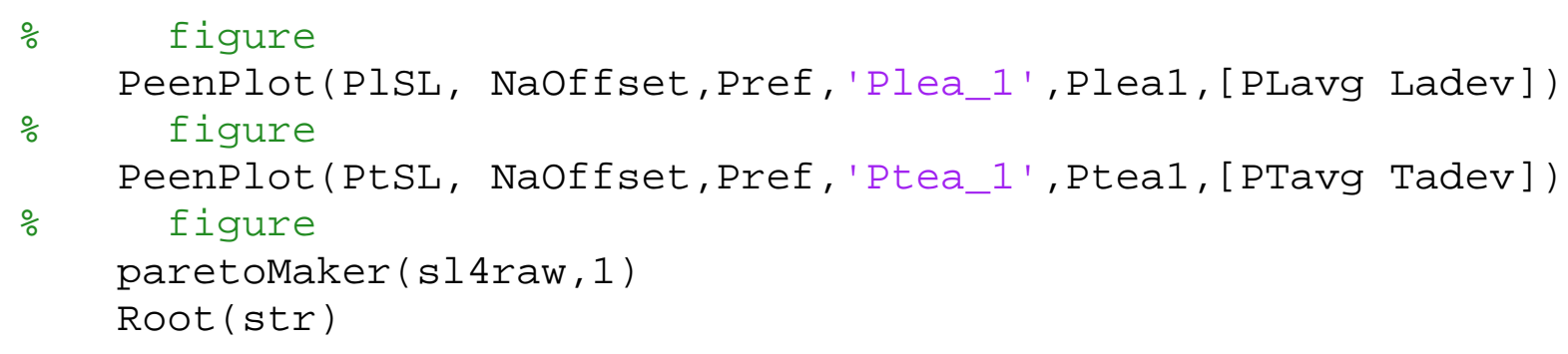

end 


\section{Standard Deviation \& Average Calculations:}

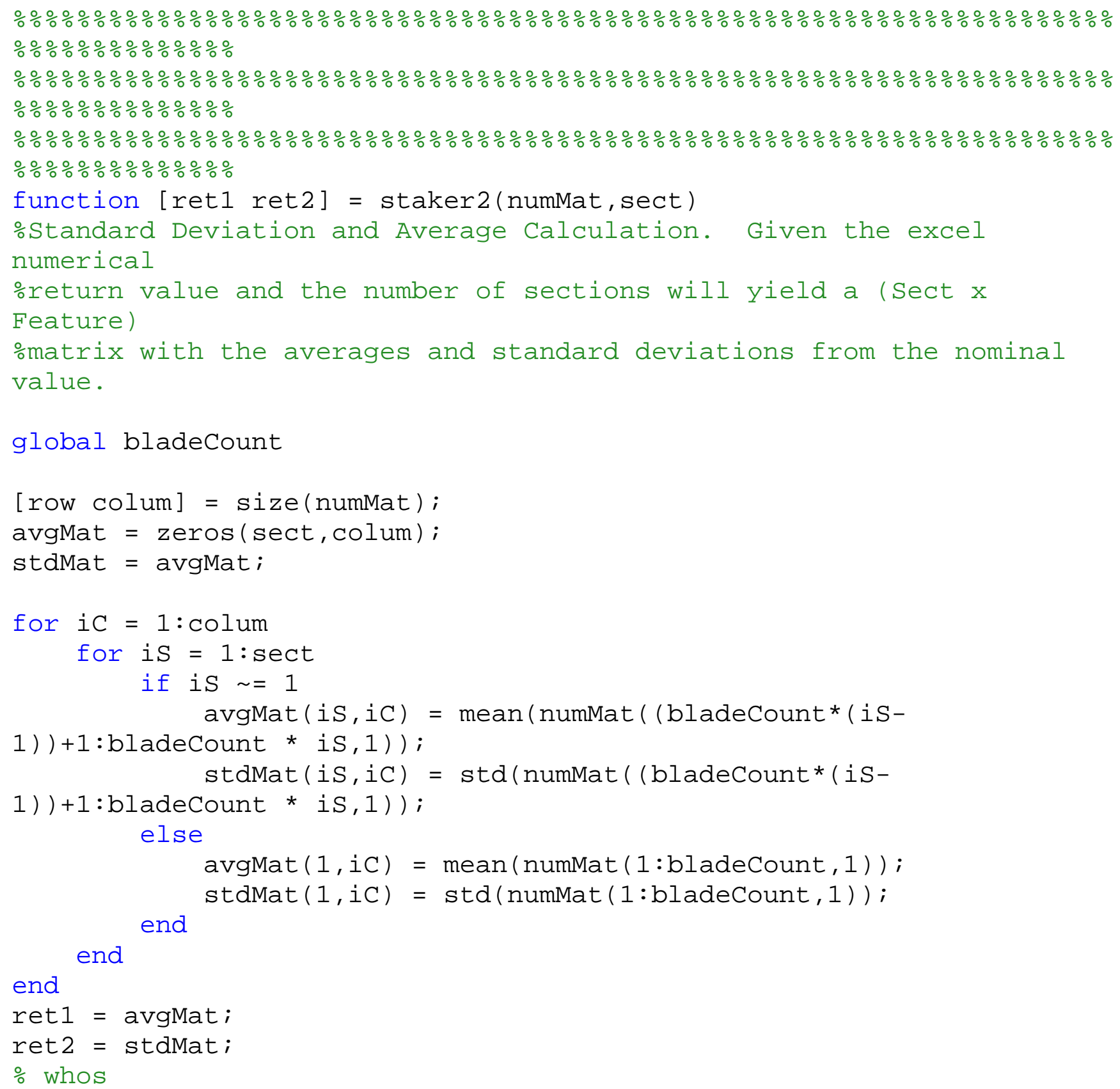




\section{DTP Calculations:}

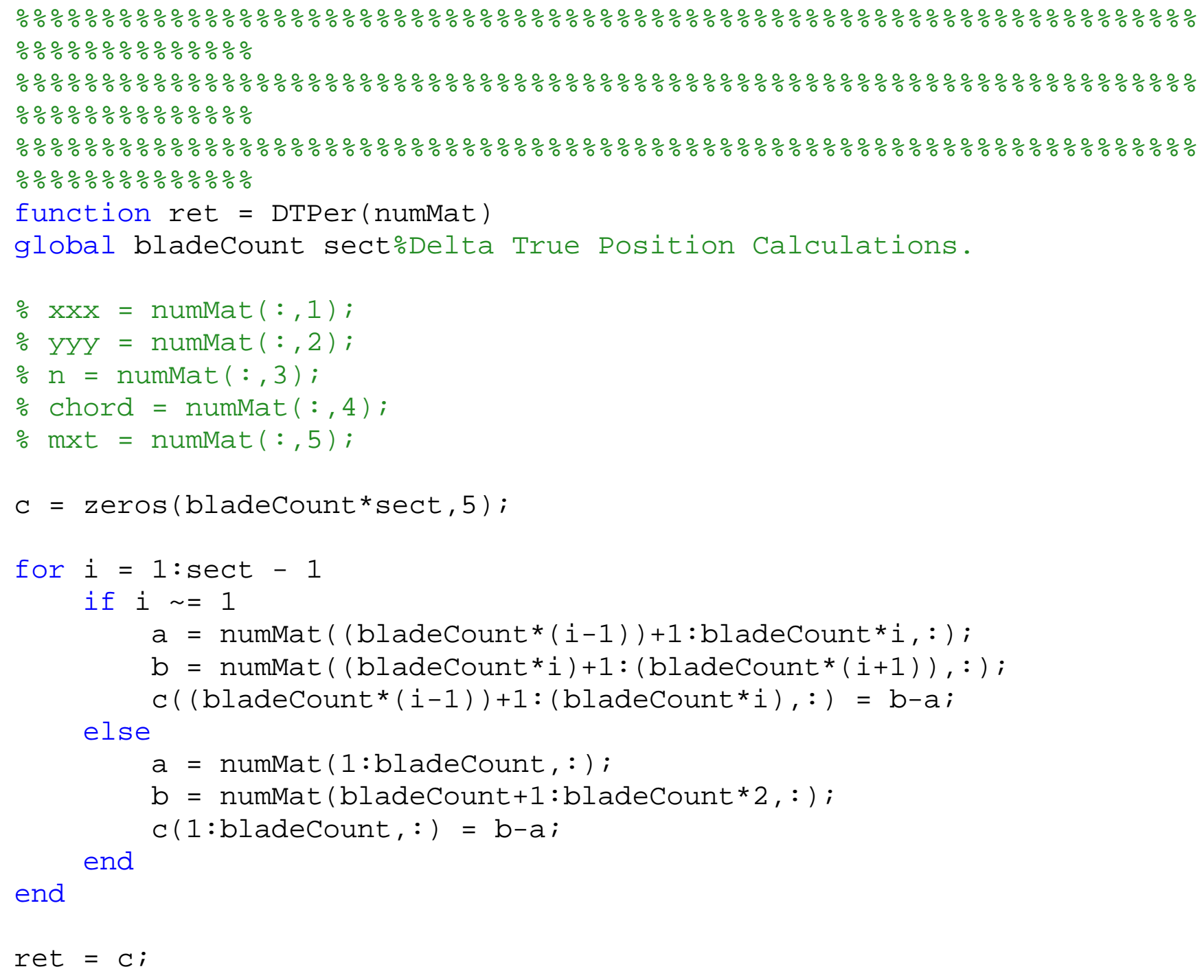




\section{Chord Loss Calculations:}

\%\%\%\%\%\%\%\%\%\%\%\%\%\%\%\%\%\%\%\%\%\%\%\%\%\%\%\%\%\%\%\%\%\%\%\%\%\%\%\%\%\%\%\%\%\%\%\%\%\%\%\%\%\%\%\%\%\%\%\%\%\%\%\% \%\%\%\%\%\%\%\%\%\%\%\%

\%\%\%\%\%\%\%\%\%\%\%\%\%\%\%\%\%\%\%\%\%\%\%\%\%\%\%\%\%\%\%\%\%\%\%\%\%\%\%\%\%\%\%\%\%\%\%\%\%\%\%\%\%\%\%\%\%\%\%\%\%\%\%\% \%\%\%\%\%\%\%\%\%\%\%\%

\%\%\%\%\%\%\%\%\%\%\%\%\%\%\%\%\%\%\%\%\%\%\%\%\%\%\%\%\%\%\%\%\%\%\%\%\%\%\%\%\%\%\%\%\%\%\%\%\%\%\%\%\%\%\%\%\%\%\%\%\%\% $\% \% \% \% \% \% \% \% \%$

function [mu sigma data] = chordCalcs (numMat)

\%chord average and Chord Loss Calculation. Plots Chord and the predicated

\%final chord deviations.

\%NOTE: This sub-function returns the average and standard deviation@ FINAL

global bladecount Cindex c_loss sect

$\mathrm{b}=[]$

\%Chord Loss Calculations

for is $=1:$ length (c_loss)

if is $\sim=1$

$a=\operatorname{numMat}(($ bladeCount $*($ is -1$))+1:$ bladeCount $*$ is, 4$)+$

c_loss (is);

$b=[b ; a]$;

else

$a=\operatorname{numMat}(1:$ bladeCount $*$ is, 4$)+c \_l o s s(i s)$;

end

$\mathrm{b}=[\mathrm{b} ; \mathrm{a}]$;

end

\%Chord Average@final

avgMat $=\operatorname{zeros}(\operatorname{sect}, 1)$;

stdMat = avgMat;

for is $=1: \operatorname{sect}$

if is $\sim=1$

$\operatorname{avgMat}($ is $)=\operatorname{mean}(b(($ bladeCount $*($ iS -1$))+1:$ bladeCount * is $))$;

else

stdMat $($ iS $)=\operatorname{std}(b(($ bladeCount* $($ iS -1$))+1:$ bladeCount * is $))$;

$\operatorname{avgMat}($ is $)=\operatorname{mean}(b(1: b l a d e C o u n t)) ;$

end

$\operatorname{stdMat}($ is $)=\operatorname{std}(b(1:$ bladecount $))$;

end

$\mathrm{mu}=$ avgMat $;$ 
sigma = stdMat;

data $=b$;

$\%$ whos 


\section{Cp \& $C p_{k}$ Calculations:}

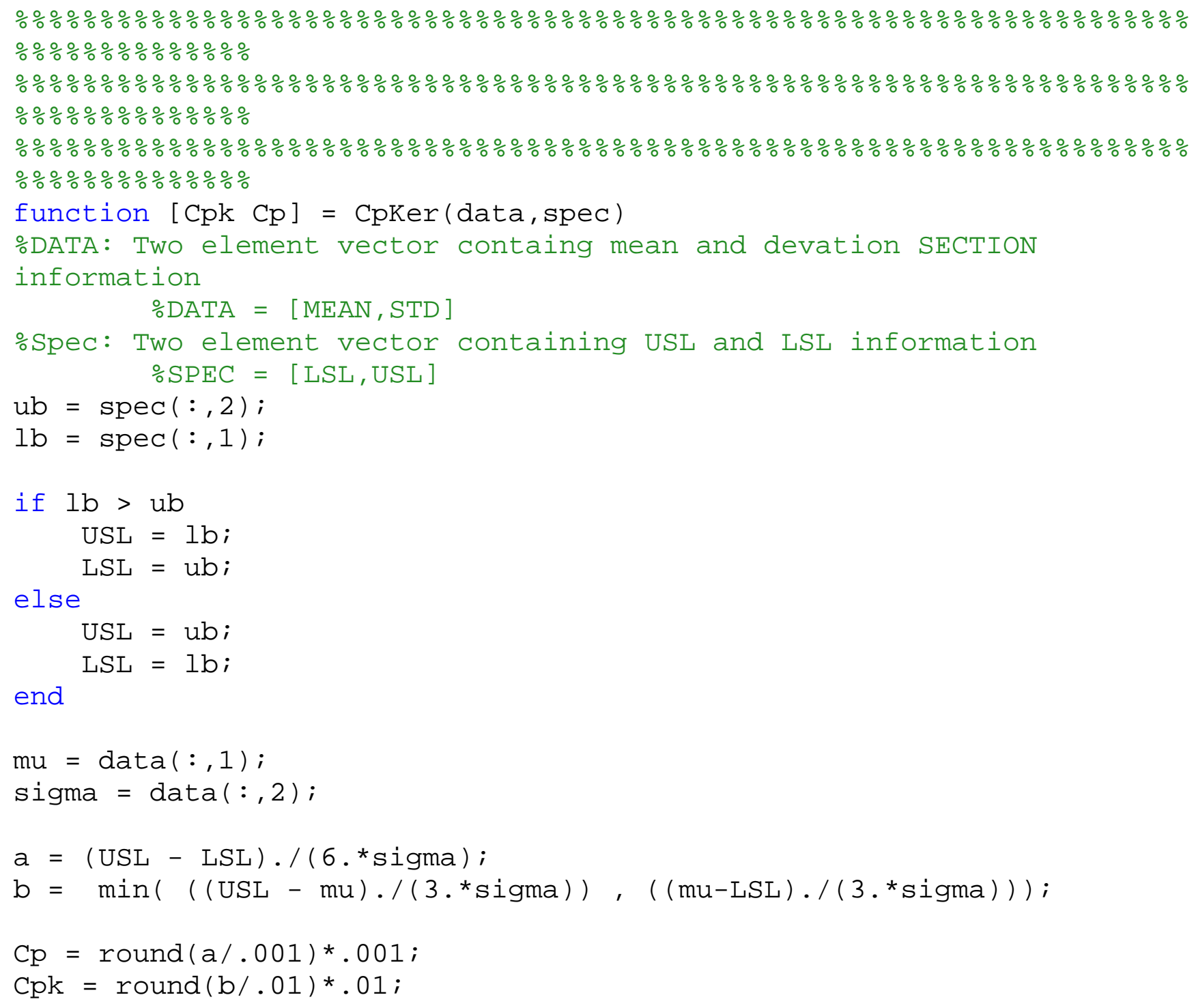




\section{Maximizer599 Calculations:}

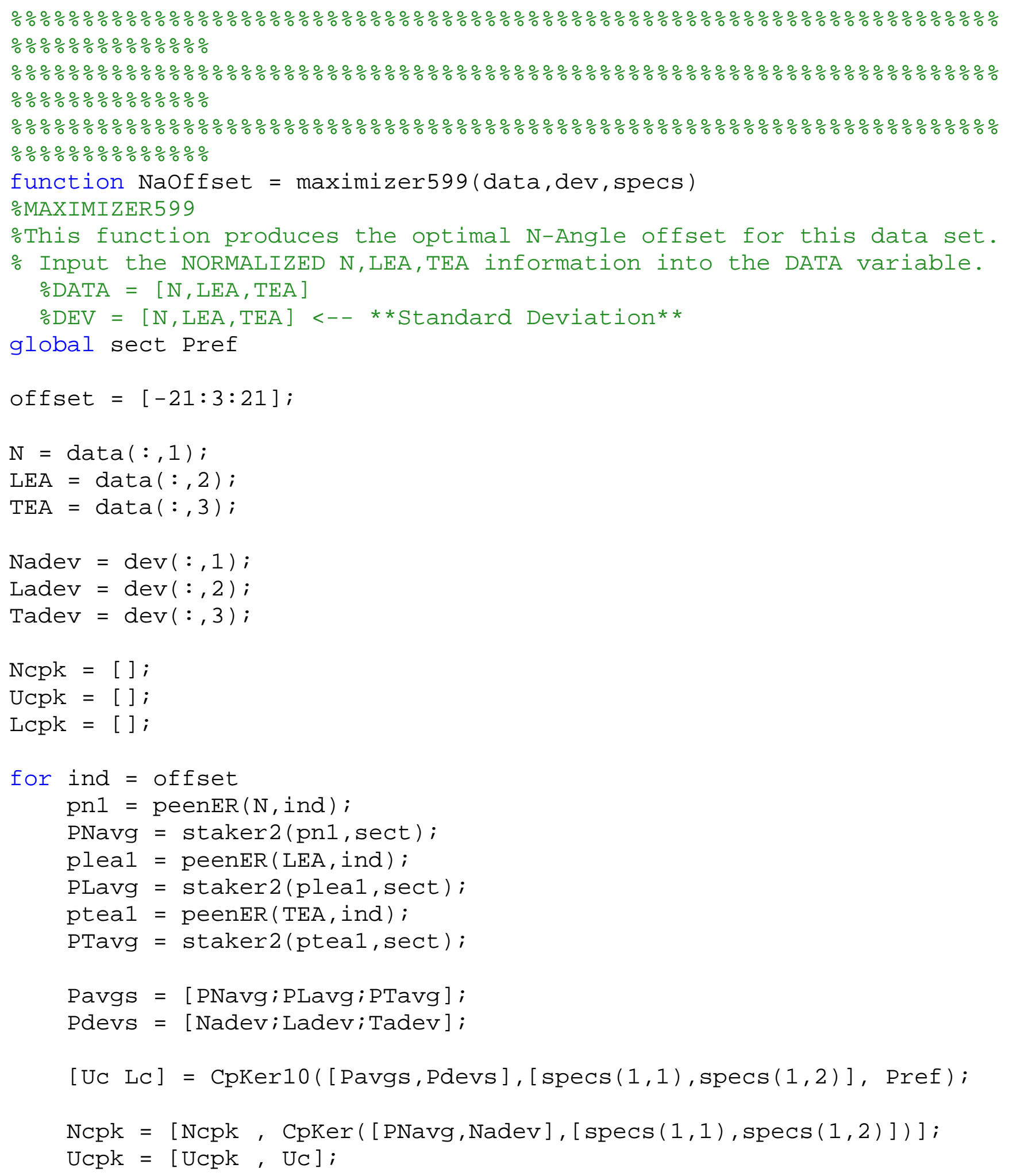


$\qquad$ Lcpk = [Lcpk, Lc $] ;$
end
plot (offset, Lcpk, offset, Ucpk)
ULdiff = abs(Ucpk - Lcpk);
maxYield = min(ULdiff);
NaOffset = offset(find(maxYield == ULdiff)); 


\section{Minimum Cpk Calculations:}

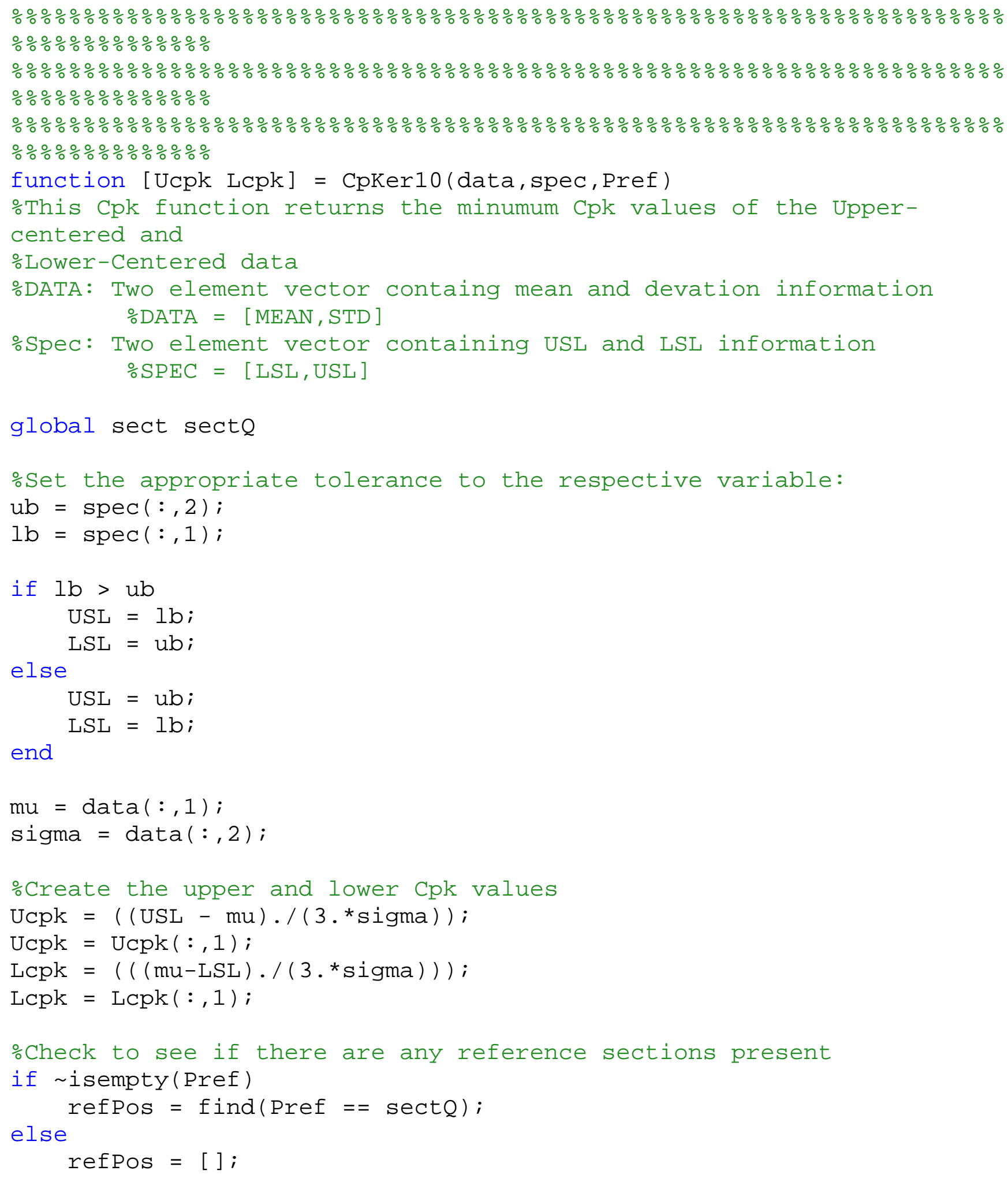


end

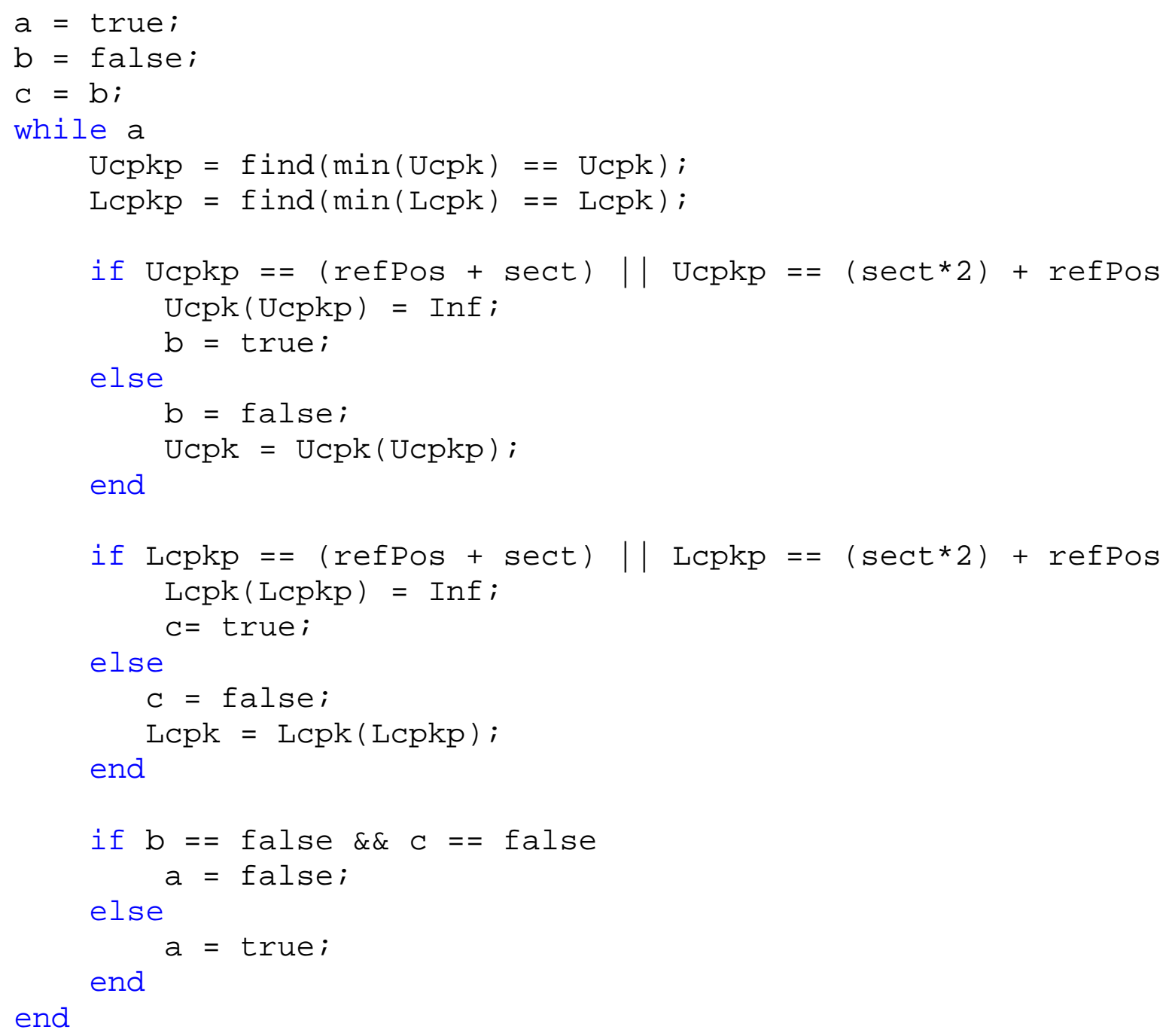




\section{Post Peen Calculations:}

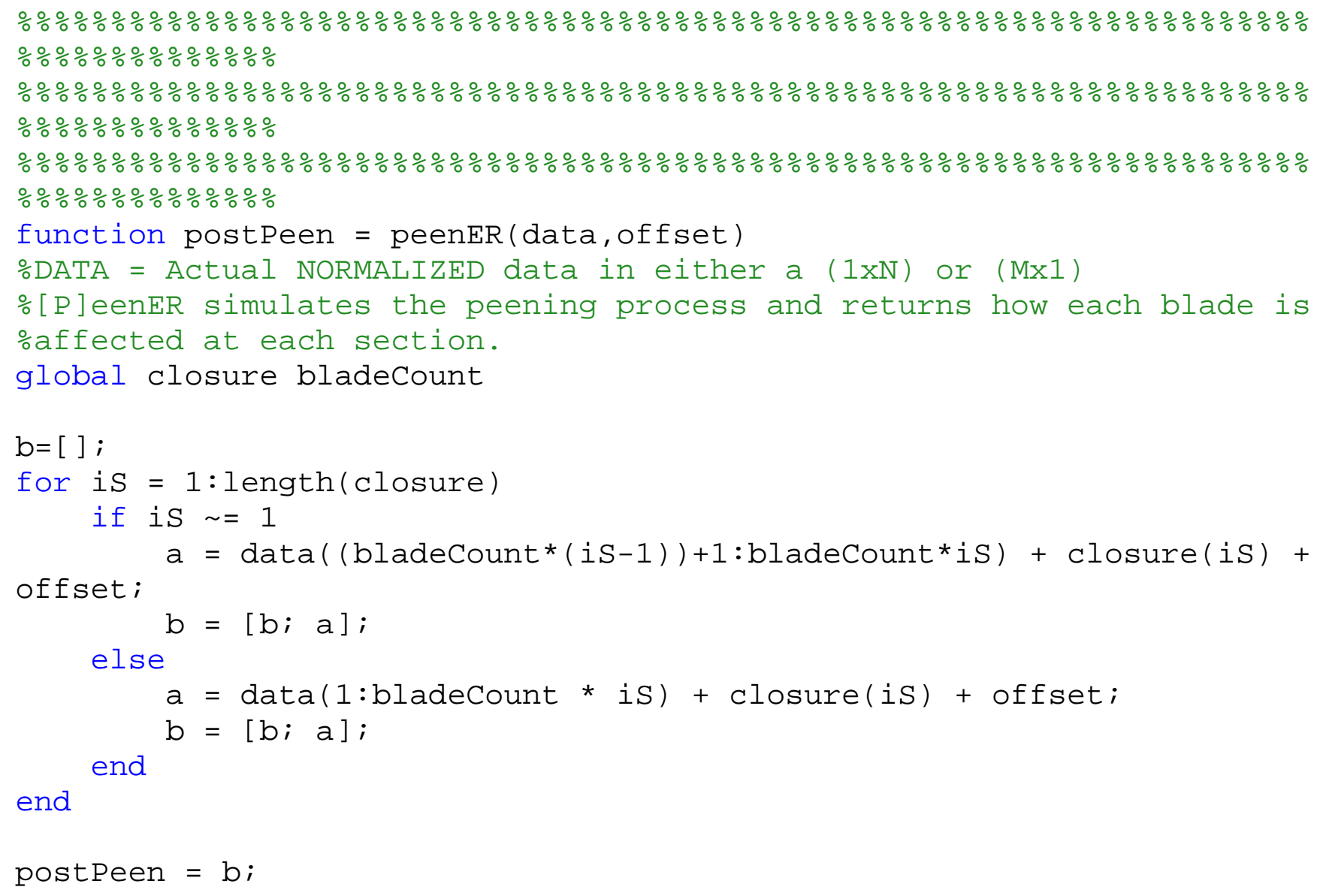




\section{Spreadsheet Maker Calculations:}

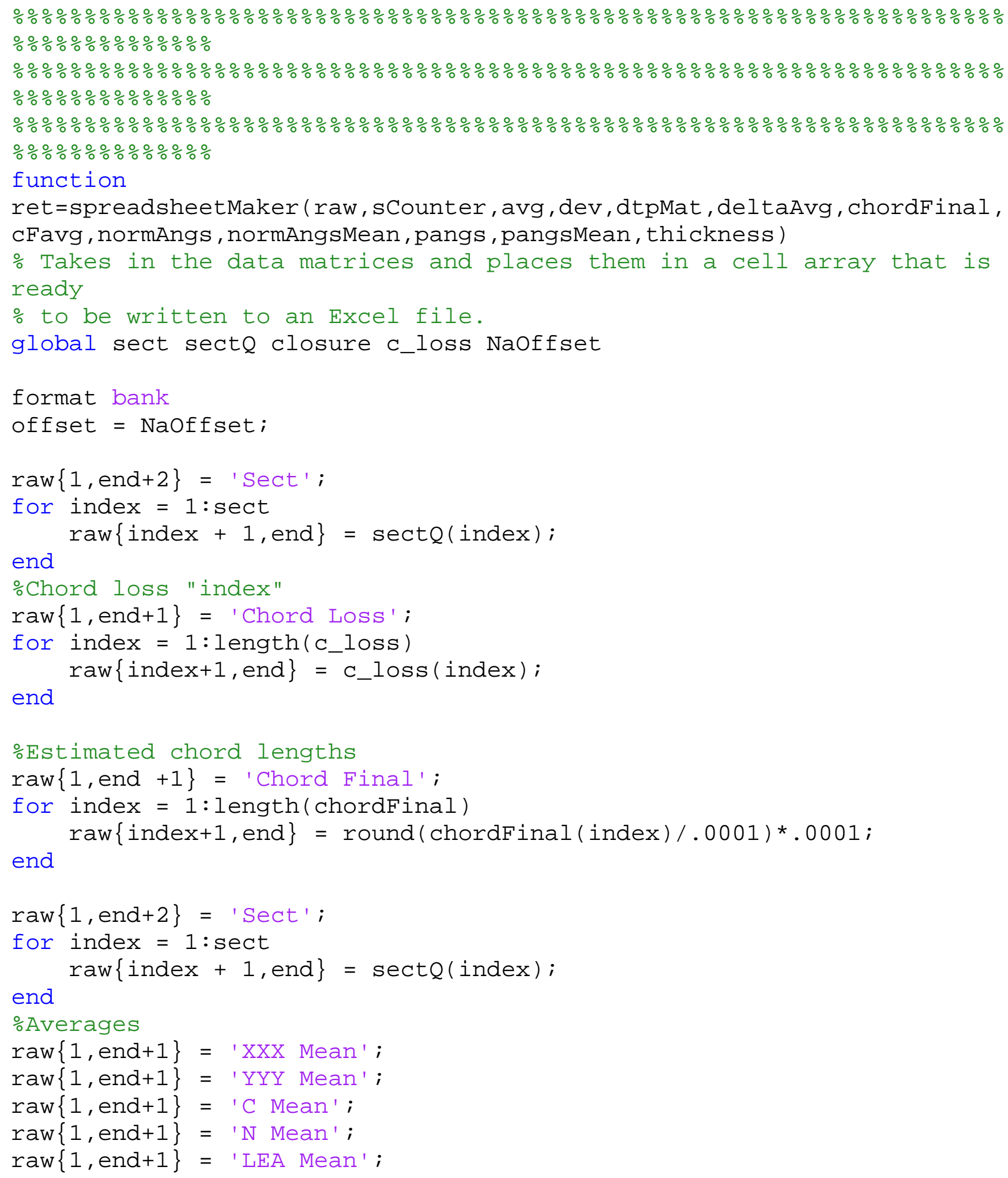




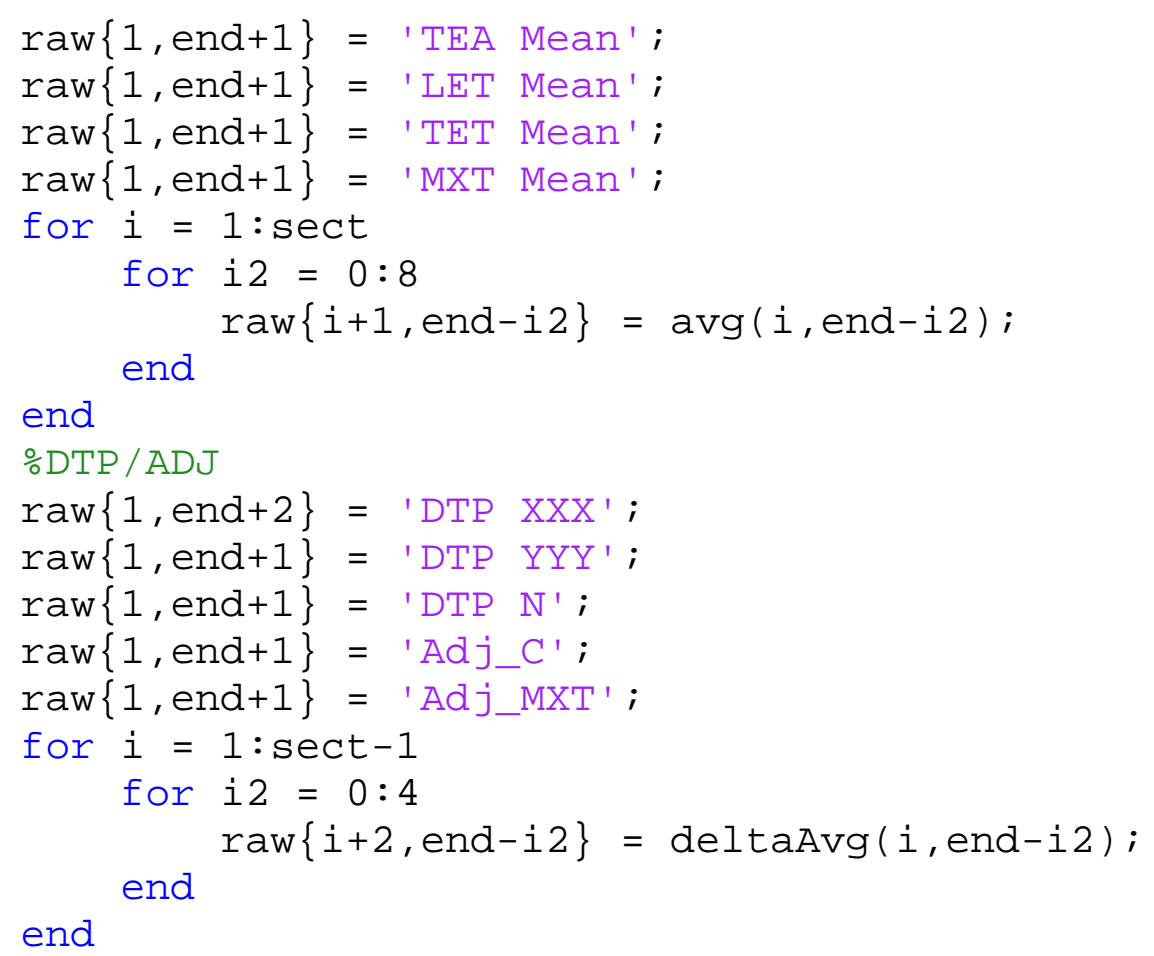


end

\%Chord@Final average - by section

$\operatorname{raw}\{1$, end +1$\}=$ 'Chord Average@ Final';

for index $=1:$ length $($ cFavg $)$

$\operatorname{raw}\{$ index +1, end $\}=\operatorname{round}(\operatorname{cFavg}($ index $) / .0001) * .0001 ;$

end

\%Normalized [N LEA TEA]

$\operatorname{raw}\{1$, end +2$\}=$ 'N_1';

$\operatorname{raw}\{1$, end +1$\}=$ 'LEA_1';

$\operatorname{raw}\{1$, end +1$\}=$ 'TEA_1';

for $i=1:$ length (normAngs)

for $i 2=0: 2$

end

$\operatorname{raw}\{i+1$, end $-i 2\}=\operatorname{round}(\operatorname{normAngs}(i$, end $-i 2) / .0001) * .0001$;

end

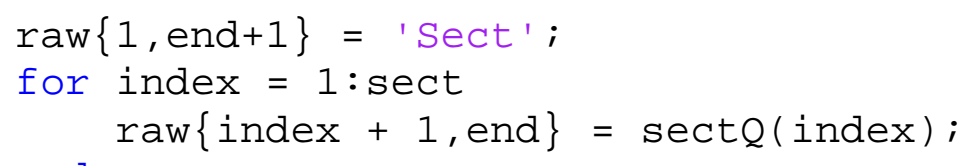

end

$\%[$ Navg LEAavg TEAavg] Averages by section

$\operatorname{raw}\{1$, end +1$\}=$ 'N_1 Mean';

$\operatorname{raw}\{1$, end +1$\}=$ 'LEA_1 Mean' ;

$\operatorname{raw}\{1$, end +1$\}=$ 'TEA_1 Mean';

for $i=1:$ length (normAngsmean)

for $i 2=0: 2$

$\operatorname{raw}\{i+1$, end $-i 2\}=\operatorname{round}(\operatorname{normAngsMean}(i$, end $-i 2) / .0001) * .0001$;

end end

\%DTP - by blade

$\operatorname{raw}\{1$, end +2$\}=$ 'DTP $X '$;

$\operatorname{raw}\{1$, end +1$\}=$ 'DTP $Y^{\prime}$;

$\operatorname{raw}\{1$, end +1$\}=$ 'DTP $N^{\prime}$;

$\operatorname{raw}\{1$, end +1$\}=$ 'ADJ $C^{\prime}$;

$\operatorname{raw}\{1$, end +1$\}=$ 'ADJ MXT';

for $i=1:$ length (dtpMat)

for $i 2=0: 4$

end

$\operatorname{raw}\{i+1$, end $-i 2\}=\operatorname{dtpMat}(i$, end $-i 2) ;$

end

$\operatorname{raw}\{1$, end +2$\}=$ 'Sect $^{\prime}$; 


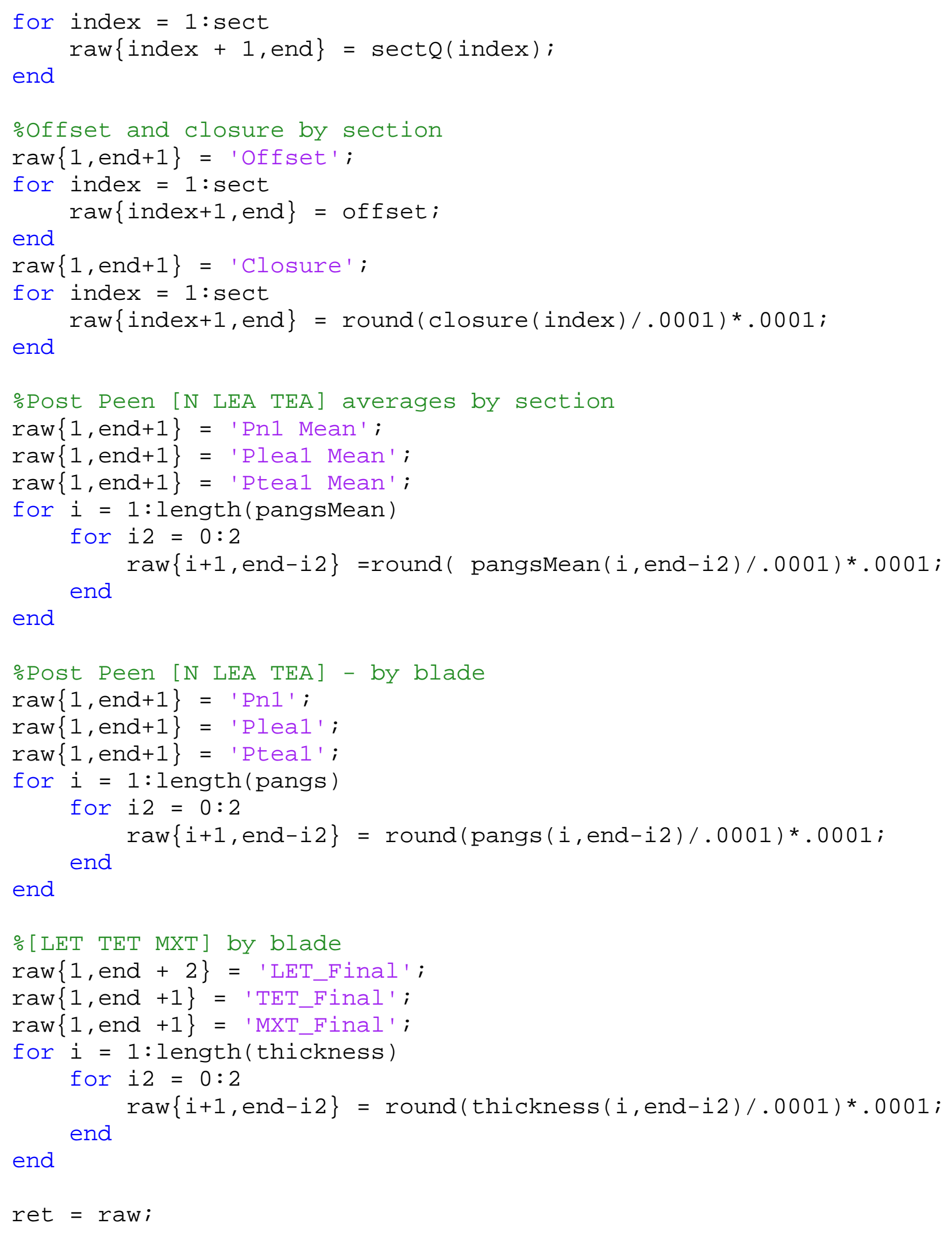




\section{Normal Plot Calculations:}

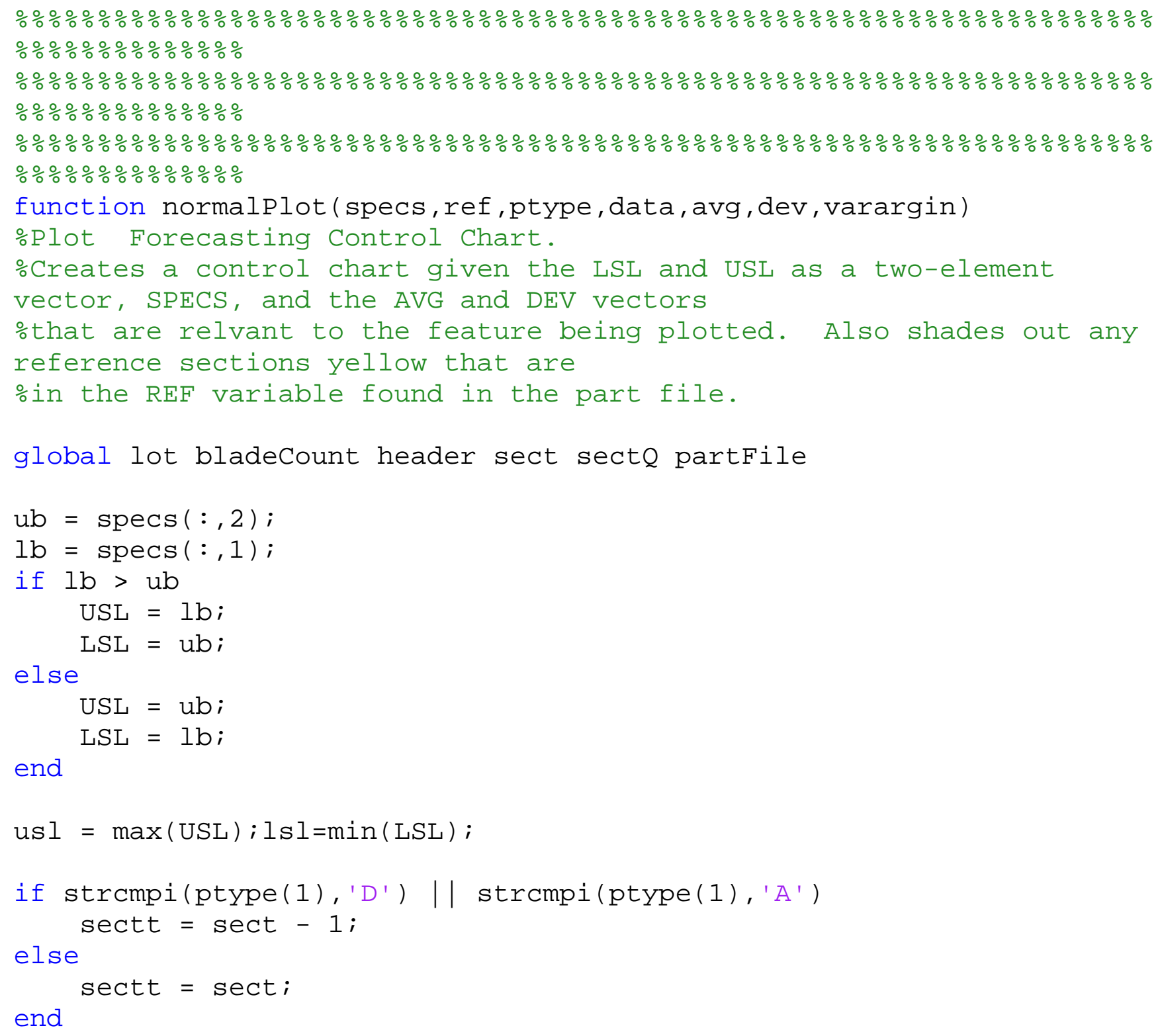




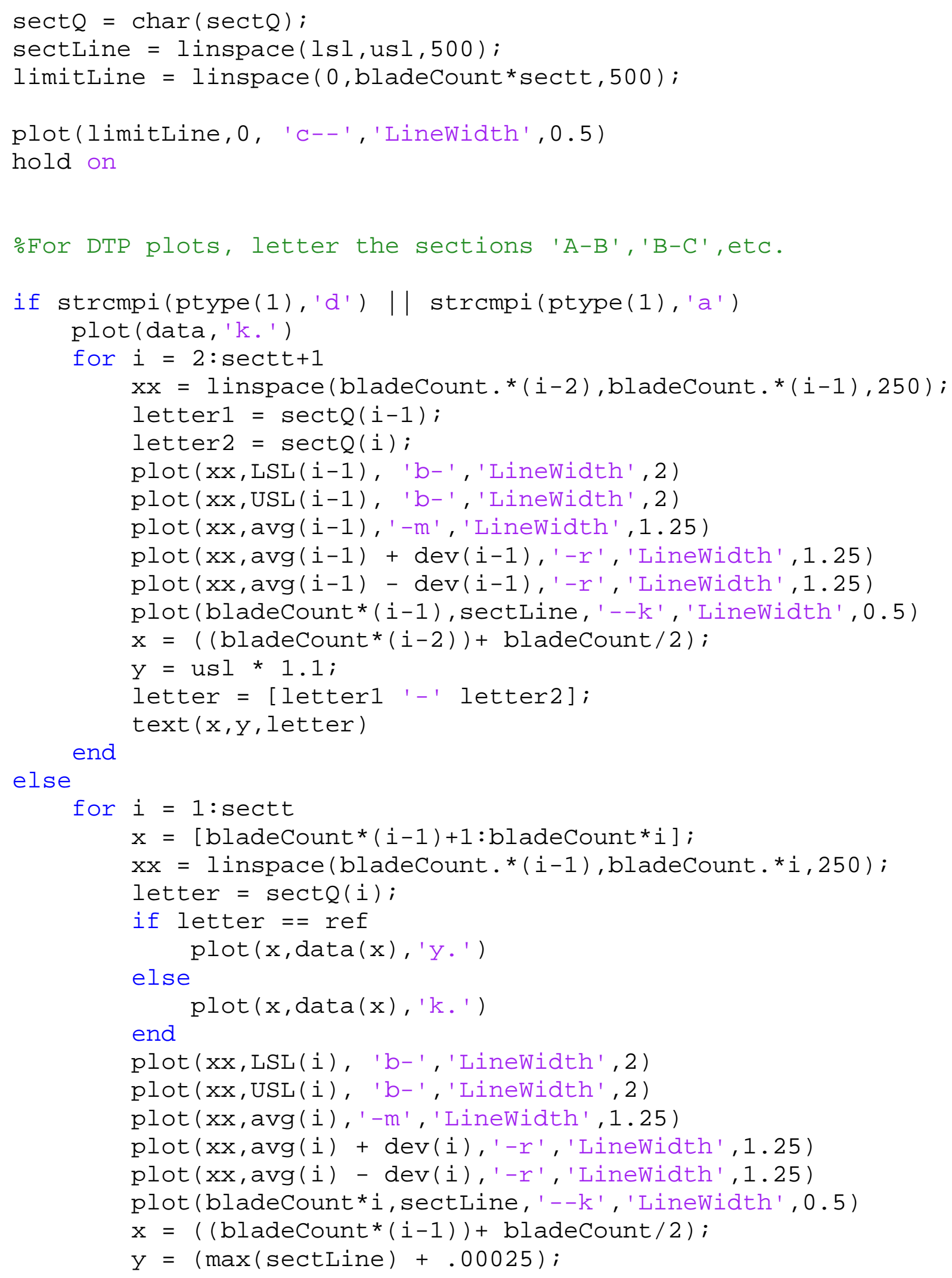


end ${ }^{\operatorname{text}(x, y, \text { letter })}$

\%Adds text to the graph

if strcmpi(ptype,' n_1') || strcmpi(ptype, 'lea_1') ||

strcmpi(ptype, 'tea_1') || strcmpi(ptype, 'DTP N') ||

strcmpi(ptype, 'CLEA') I| strcmpi(ptype, 'CTEA')

ylabel('Deviation from nominal (min.)'); xlabel('Observation Number' )

else Number' )

ylabel('Deviation from nominal (in.)'); xlabel('Observation

end

$\mathrm{bc}=$ num2str (bladeCount);

str2 = ['LPI Inspections lot ' lot ' (Basis:' bc ' parts) JB43'];

\%create title for graph

if -isempty(varargin)

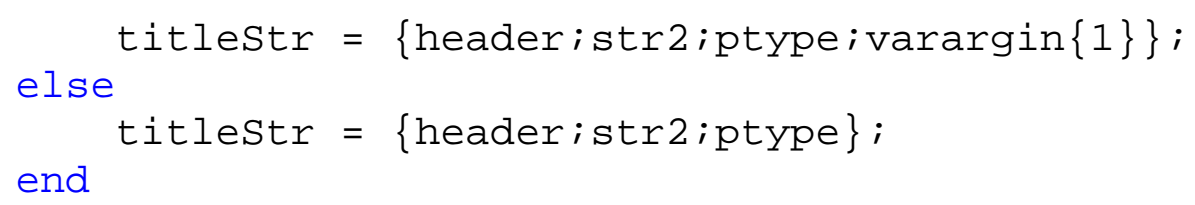

clear str2 str1 bc \%Free up some space and variables

clear str2 str1 bc \%Free up some space and variables

title(titlestr)

text (bladeCount *sectt, usl + ((usl*.25)/2), 'USL')

text (bladeCount * sectt, lsl + ((lsl*.25)/2), 'LSL')

\%Re-sizes the graph

if $\max ($ data $)<$ usl \&\& $\min ($ data $)>1 s l$

set (gca, 'xlim', [0 (length(data)+length(data $\left.\left.\left.{ }^{*} .05\right)\right]\right)$

else set (gca,'ylim', [(lsl + lsl*.05) (usl + usl*.05)])

set (gca, 'xlim', [0 (length(data)+length(data)*.05)] )

end $\operatorname{set}\left(\right.$ gca, 'ylim', [(lsl+min (data)*.5) (usl+max (data) $\left.\left.\left.{ }^{*} .5\right)\right]\right)$

hold off

\%save the graph toa jpeg file

\%build filename 
filename $=$ [partFile '_' lot '_' ptype '.jpg']; print('-djpeg50', filename); 


\section{Post Peen Plot Calculations:}

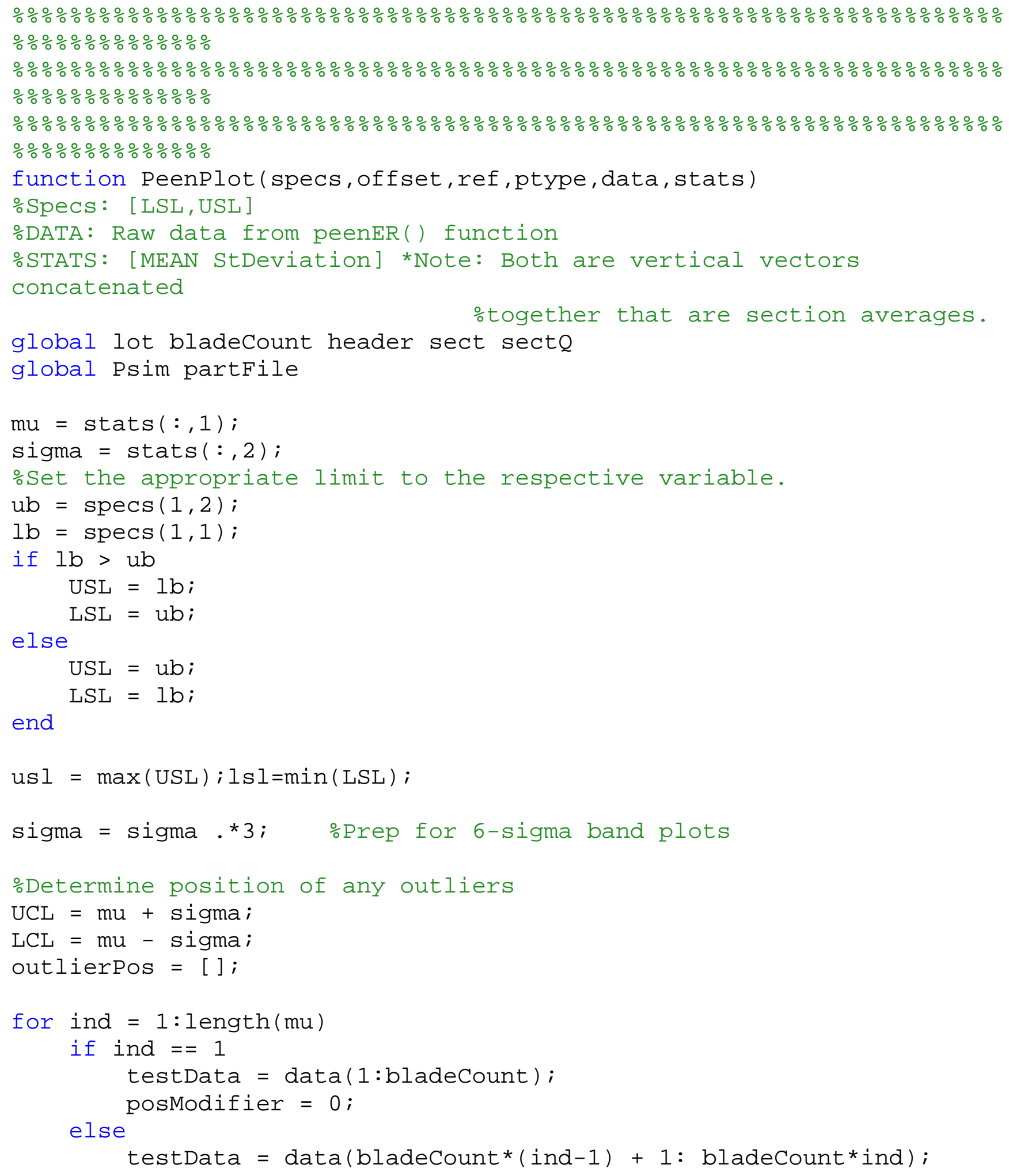




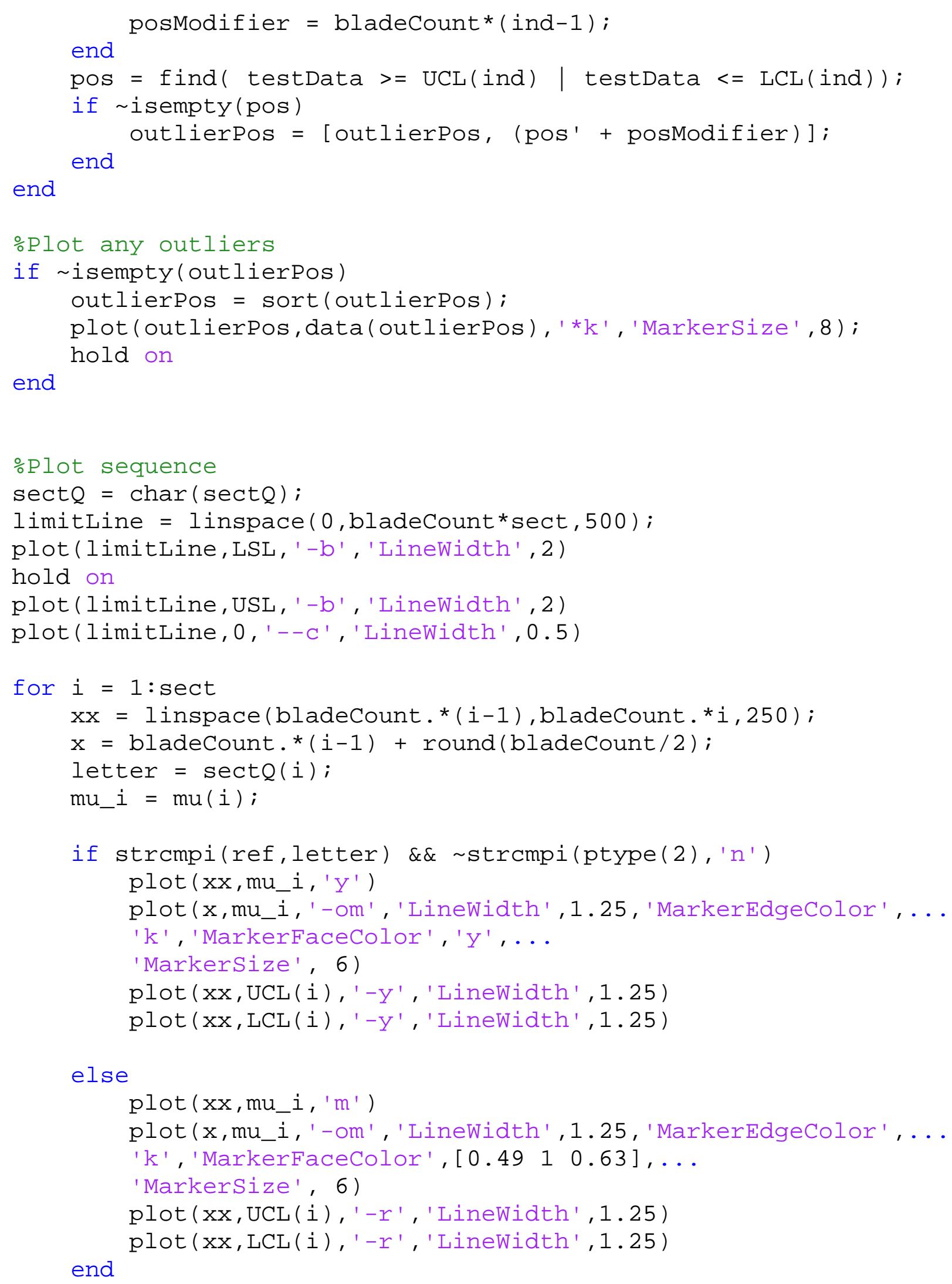




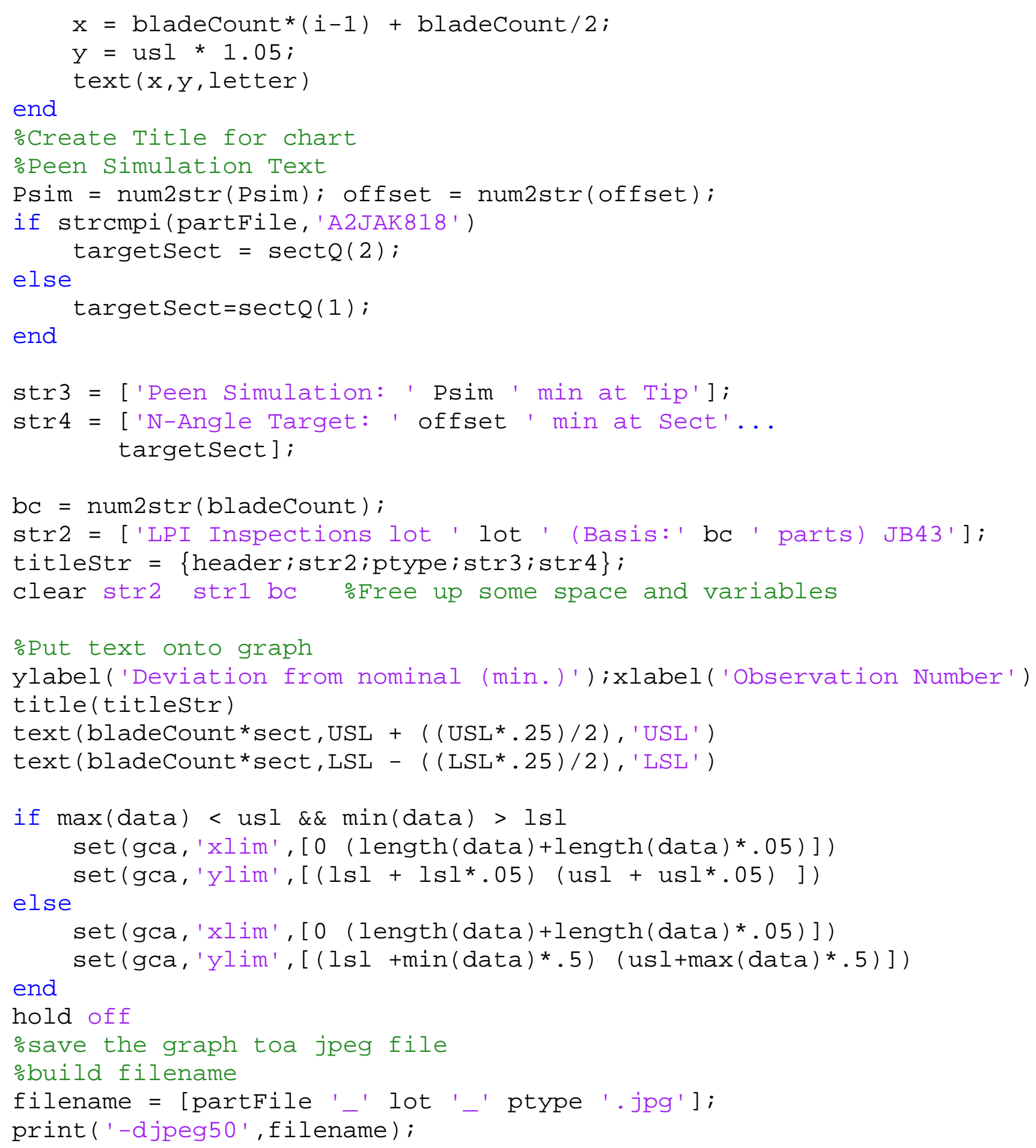




\section{DTP Calculations:}

\%\%\%\%\%\%\%\%\%\%\%\%\%\%\%\%\%\%\%\%\%\%\%\%\%\%\%\%\%\%\%\%\%\%\%\%\%\%\%\%\%\%\%\%\%\%\%\%\%\%\%\%\%\%\%\%\%\%\%\%\%\%\%\%\% \%\%\%\%\%\%\%\%\%\%\%\%

\%\%\%\%\%\%\%\%\%\%\%\%\%\%\%\%\%\%\%\%\%\%\%\%\%\%\%\%\%\%\%\%\%\%\%\%\%\%\%\%\%\%\%\%\%\%\%\%\%\%\%\%\%\%\%\%\%\%\%\%\%\%\%\% \%\%\%\%\%\%\%\%\%\%\%\%

\%\%\%\%\%\%\%\%\%\%\%\%\%\%\%\%\%\%\%\%\%\%\%\%\%\%\%\%\%\%\%\%\%\%\%\%\%\%\%\%\%\%\%\%\%\%\%\%\%\%\%\%\%\%\%\%\%\%\%\%\%\%\%\%

$\% \% \% \% \% \% \% \% \%$

function paretoMaker(specs, count)

\%rawCellarr = [xxx, yyy, cF, pn1, plea1, ptea1, let_f, tet_f,mxt_f, lep, tep, psp, ssp] \%SPECS is in the same order as MAT but contains the USL and LSL \%Function searches throught the data for blades out of tolerance $\%$ and creates a list of the defects and the measurement. global defectcount names header global bladecount sect lot partfile

filename $=[$ partFile ' $'$ lot '_rawData.xls'] ;
$[$ num txt raw] =xlsread (filename, 'Raw Data');

$\operatorname{col}=[2: 3,22,75: 77,79: 81,15,17,16,18]$;

posMod $=7$;

defectcount $=[]$;

names $=\{\}$;

dc $=[]$;

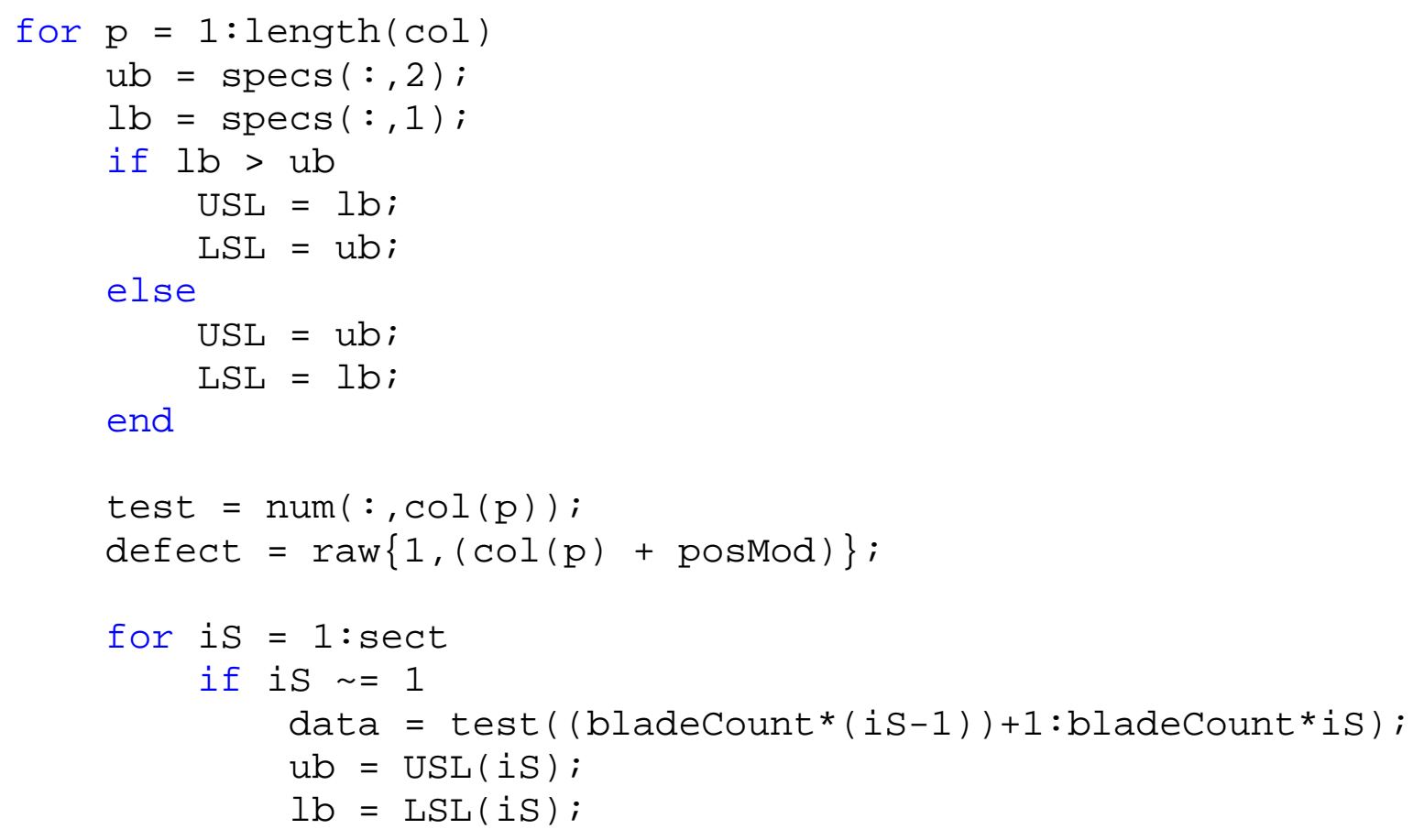




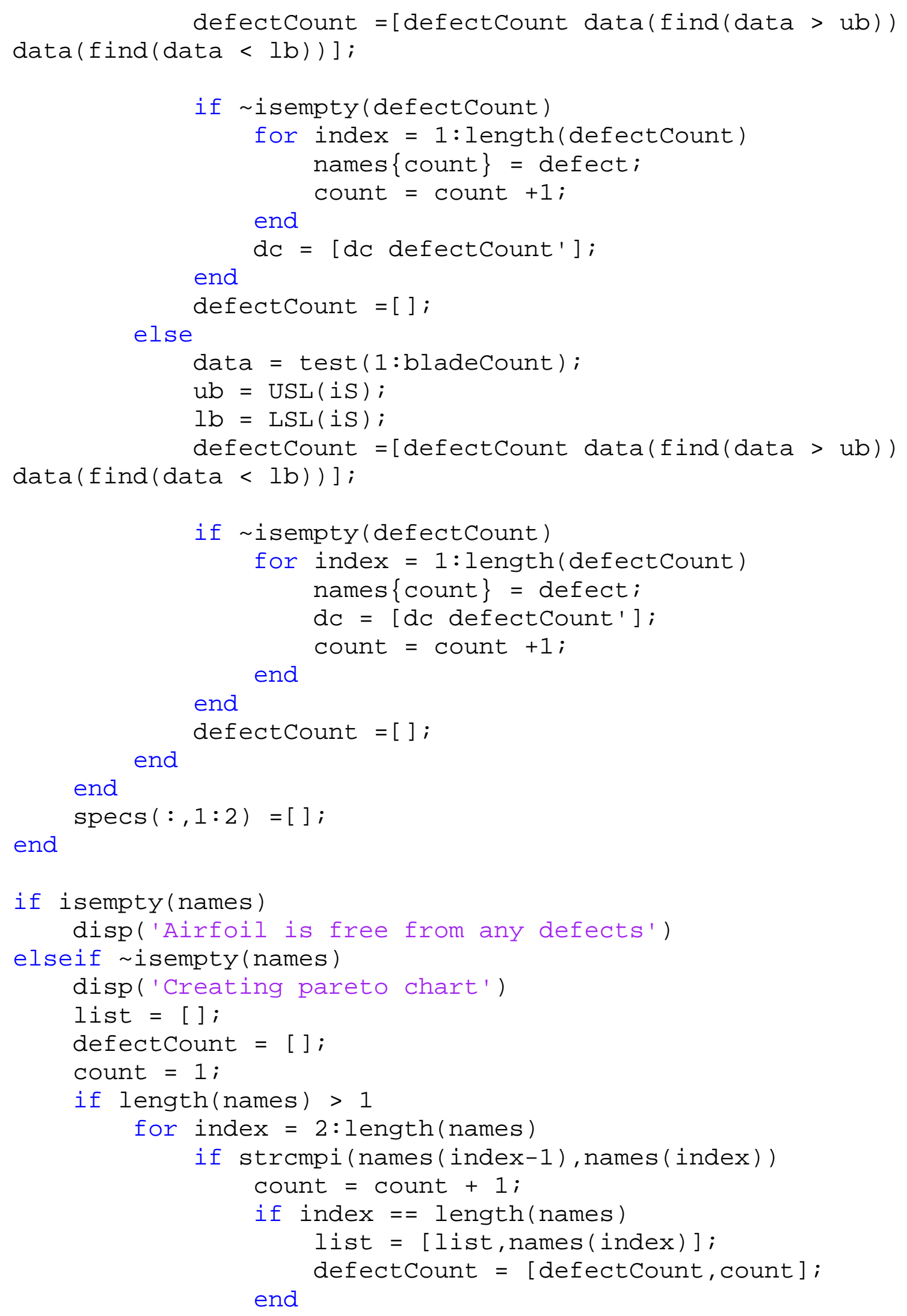




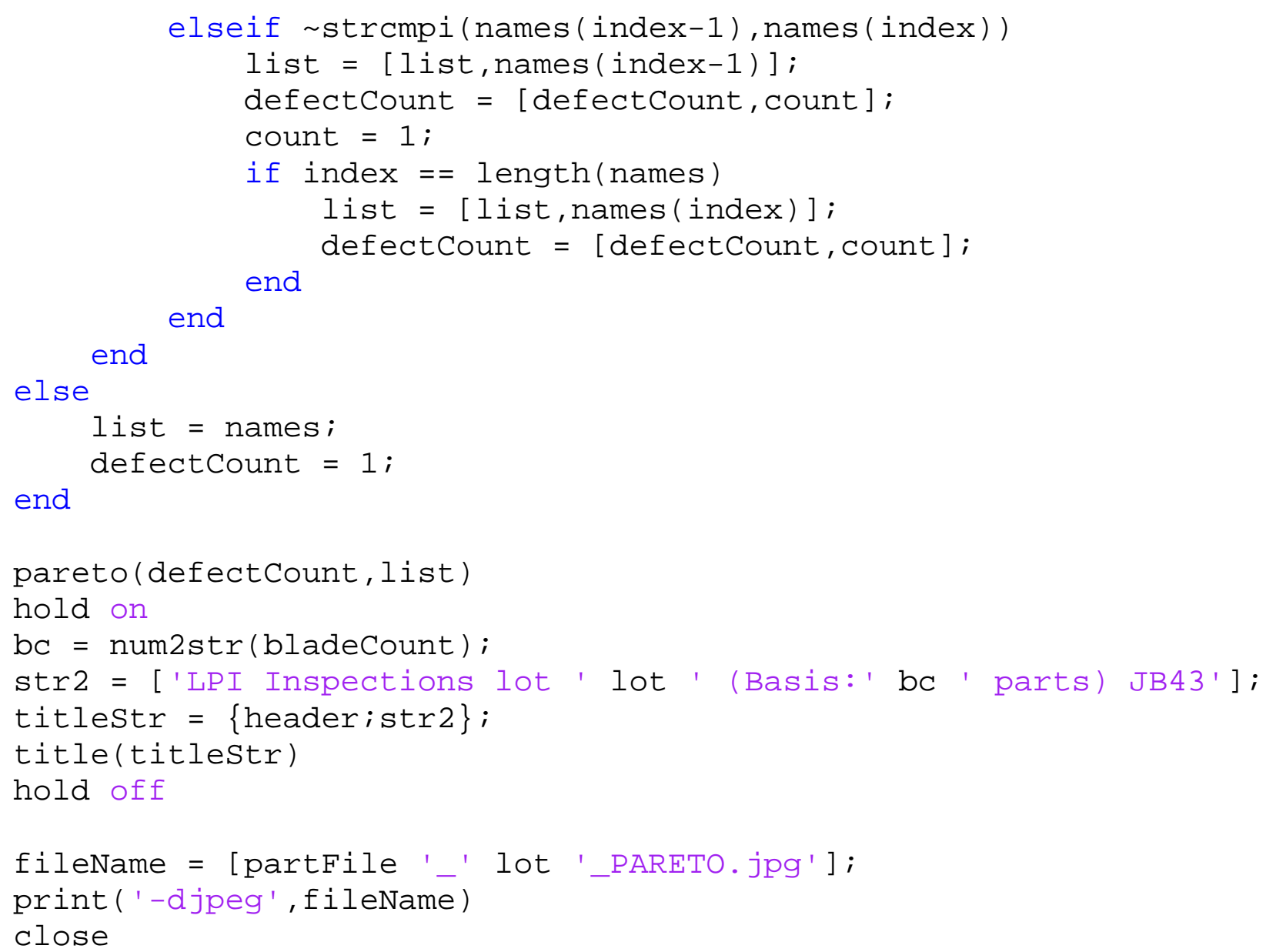




\section{Root Calculations:}

\%\%\%\%\%\%\%\%\%\%\%\%\%\%\%\%\%\%\%\%\%\%\%\%\%\%\%\%\%\%\%\%\%\%\%\%\%\%\%\%\%\%\%\%\%\%\%\%\%\%\%\%\%\%\%\% \%\%\%\%\%\%\%\%\%\%\%

\%\%\%\%\%\%\%\%\%\%\%\%\%\%\%\%\%\%\%\%\%\%\%\%\%\%\%\%\%\%\%\%\%\%\%\%\%\%\%\%\%\%\%\%\%\%\%\%\%\%\%\%\%\%\%\%\% \%\%\%\%\%\%\%\%\%\%\%

\%\%\%\%\%\%\%\%\%\%\%\%\%\%\%\%\%\%\%\%\%\%\%\%\%\%\%\%\%\%\%\%\%\%\%\%\%\%\%\%\%\%\%\%\%\%\%\%\%\%\%\%\%\%\%\%\% $\% \% \% \% \% \% \% \% \%$

function Root(str)

global Ftol Ptol lot partFile header bladecount [num1 t1 raw1] = xlsread (str, 'Sheet2'); clear t1

$\%$ create a check to see if data is present

if isempty(num1)

disp('No Fillet or Platform data is present') return

end

$\left[\begin{array}{ll}r & c\end{array}\right]=\operatorname{size}($ raw 1$)$

section $=\operatorname{raw} 1\{1,2\}$;

count $=1$;

queue $=\{\}$;

qCount $=[]$;

for index $=2: r$

test $=\operatorname{raw} 1\{$ index, 2$\}$;

if strcmpi(test, section)

count $=$ count +1 ;

if index $==r$

queue $\{$ end +1$\}=$ section;

end

qCount $=[$ qCount, count $]$;

elseif strcmpi(test, section)

queue $\{$ end +1$\}=$ section;

qCount $=$ [qCount, count $]$;

count $=1$;

section = test;

end

end

data $=\operatorname{num1}(:, 4)$;

sectIndex $=$ linspace $(\odot, r$, length $($ queue $)+1)$;

limitLine $=$ linspace $(\Theta$, sectIndex $($ end $), r * 2)$;

sectLine $=$ linspace $(\Theta$, Ftol, $(1 /$ Ftol $) * 3)$; 


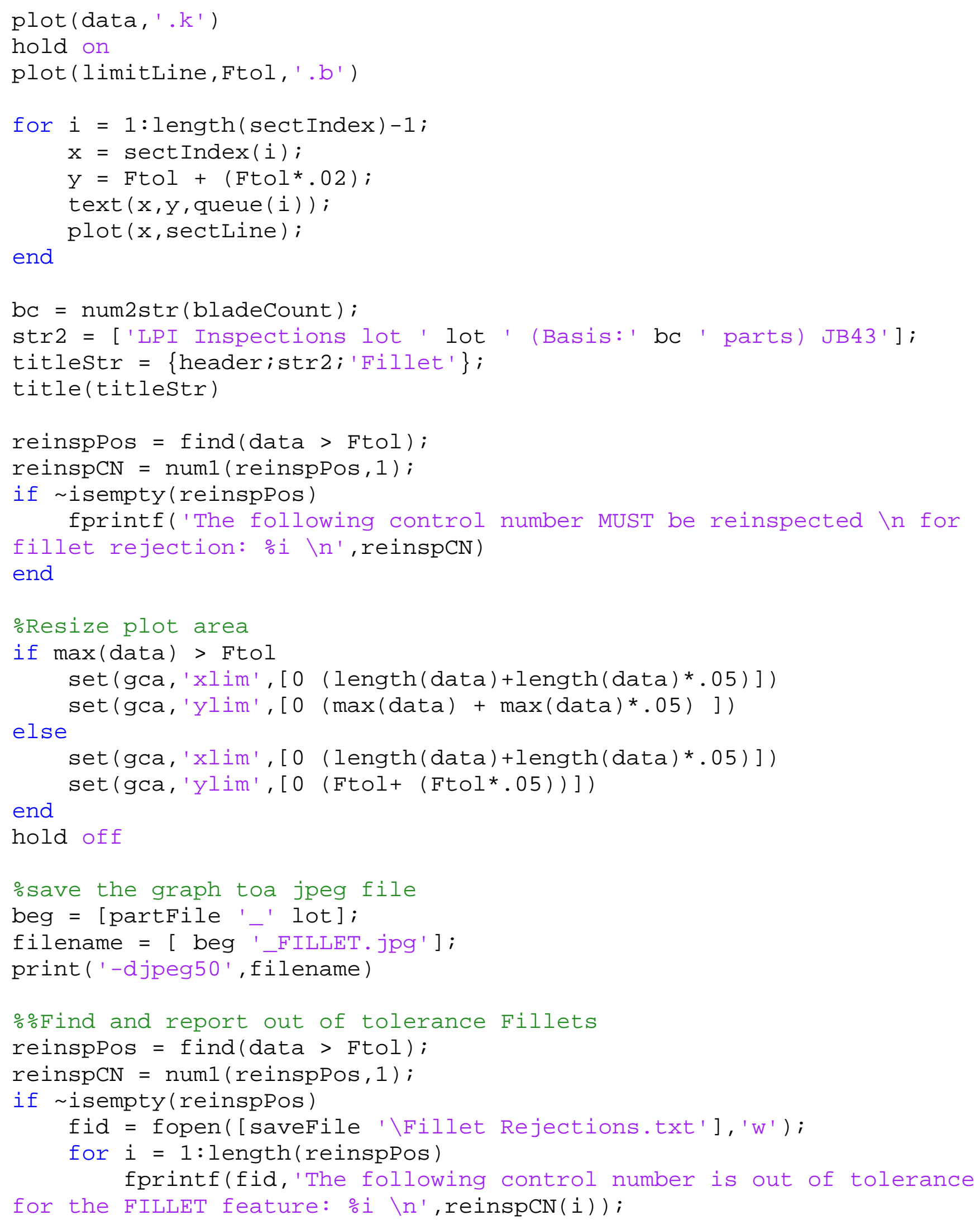




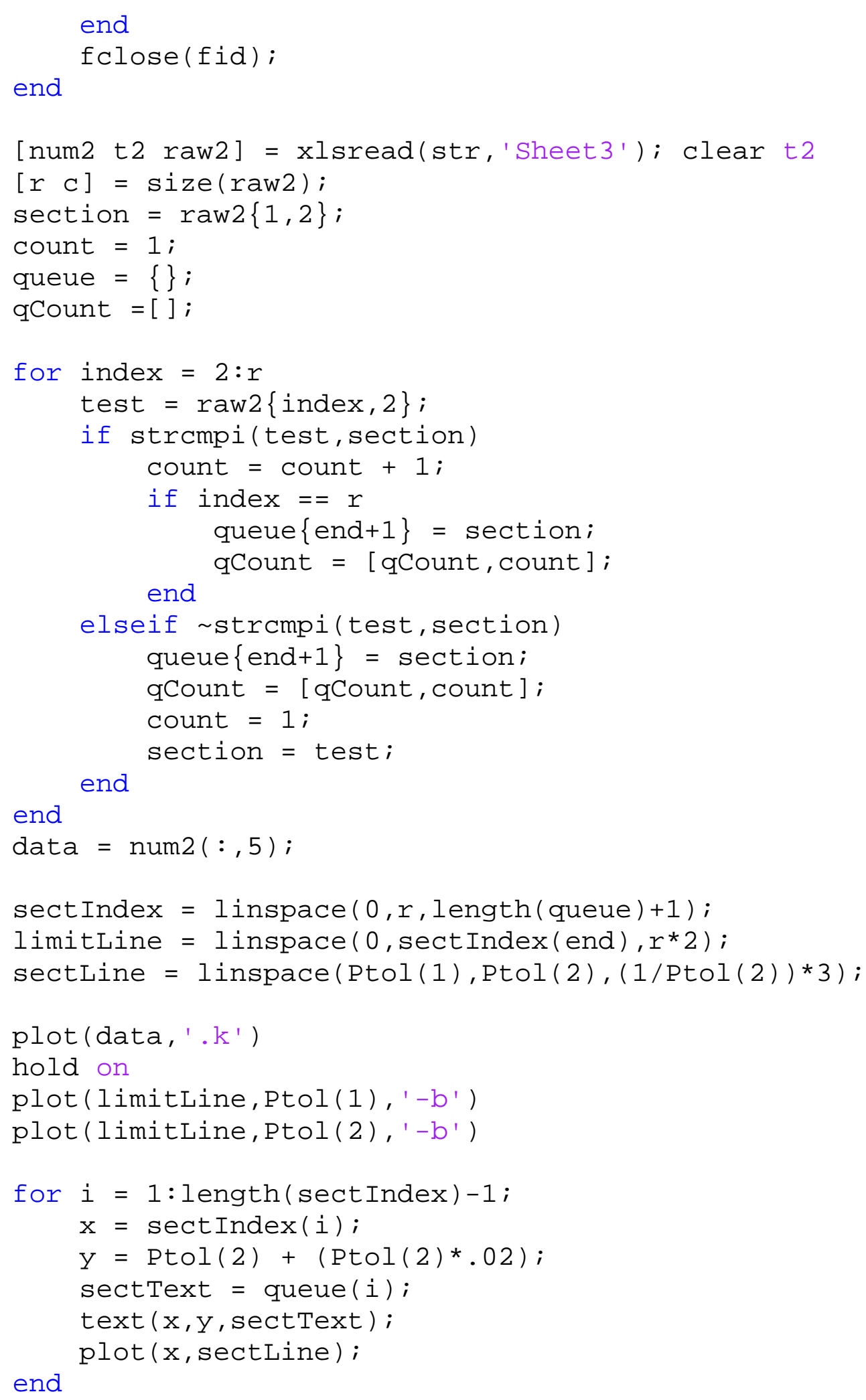




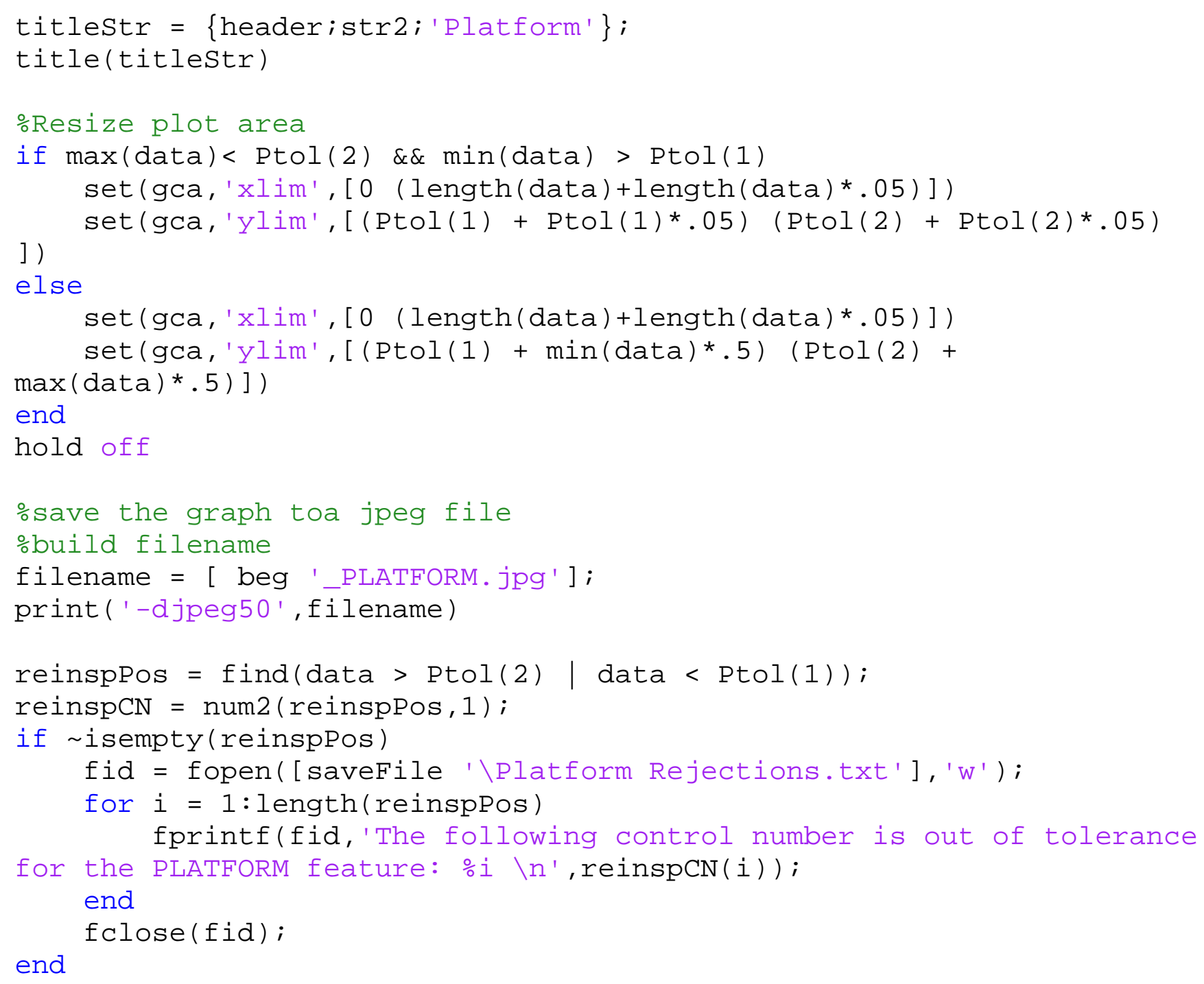




\section{Supporting Functions:}

function [mu sigma data] = chordCalcs (numMat)

\%chord average and Chord Loss Calculation. Plots chord and the predicated

\%final chord deviations.

\%NOTE: This sub-function returns the average and standard deviation@ FINAL

global bladeCount Cindex c_loss sect

disp('Calculating Chord Loss')

$\mathrm{b}=[]$

\%Chord Loss Calculations

for is $=1:$ length (c_loss)

if is $\sim 1$

$a=\operatorname{numMat}(($ bladeCount $*($ iS -1$))+1:$ bladeCount*is, 4$)+$

c_loss(is);

$$
\begin{aligned}
& b=[b ; a] ; \\
& \text { else } \\
& a=\operatorname{numMat}(1: \text { bladecount *is, } 4)+c \_ \text {loss }(i S) ; \\
& b=[b ; a] ; \\
& \text { end }
\end{aligned}
$$

end

\%Chord Average@final

avgMat $=\operatorname{zeros}(\operatorname{sect}, 1)$;

stdMat = avgMat;

for is $=1$ :sect

if is $\sim=1$ $\operatorname{avgMat}($ is $)=\operatorname{mean}(b(($ bladeCount $*($ iS -1$))+1:$ bladeCount * iS $))$; else $\operatorname{stdMat}($ is $)=\operatorname{std}(b(($ bladeCount* $($ is -1$))+1:$ bladeCount * is $))$;

$\operatorname{avgMat}($ is $)=\operatorname{mean}(b(1: b l$ adeCount $)) ;$

end end $\operatorname{stdMat}($ is $)=\operatorname{std}(b(1:$ bladeCount $)) ;$

$\mathrm{mu}=$ avgMat;

sigma = stdMat;

data $=b$; 
$\%$ whos

function [Ucpk Lcpk] = CpKer10(data, spec, Pref)

\%This cpk function returns the minumum Cpk values of the Uppercentered and

\%Lower-Centered data

\%DATA: Two element vector containg mean and devation information $\%$ DATA $=[$ MEAN, STD $]$

\%Spec: Two element vector containing USL and LSL information $\%$ SPEC $=[L S L, U S L]$

global sect sectQ

\%Set the appropriate tolerance to the respective variable:

$\mathrm{ub}=\operatorname{spec}(:, 2)$;

$1 b=\operatorname{spec}(:, 1)$;

if $l b>u b$

$\mathrm{USL}=1 \mathrm{~b} ;$

$\mathrm{LSL}=\mathrm{ub}$;

else

$\mathrm{USL}=\mathrm{ub}$;

end

$L S L=1 b ;$

$\mathrm{mu}=\operatorname{data}(:, 1) ;$

sigma $=\operatorname{data}(:, 2)$;

\%create the upper and lower cpk values

Ucpk = ( (USL $-\mathrm{mu}) \cdot /\left(3 \cdot{ }^{*}\right.$ sigma $\left.)\right)$;

Ucpk $=\operatorname{Ucpk}(:, 1)$;

Lcpk $=\left(\left((\right.\right.$ mu-LSL $) \cdot /\left(3 \cdot{ }^{*}\right.$ sigma $\left.\left.)\right)\right)$;

$\operatorname{Lcpk}=\operatorname{Lcpk}(:, 1)$;

\%check to see if there are any reference sections present

if -isempty(Pref)

refPos $=$ find $($ Pref $==$ sect $Q)$;

else

end

refpos $=[]$;
$a=$ true;
$\mathrm{b}=\mathrm{false}$;
$c=b$; 


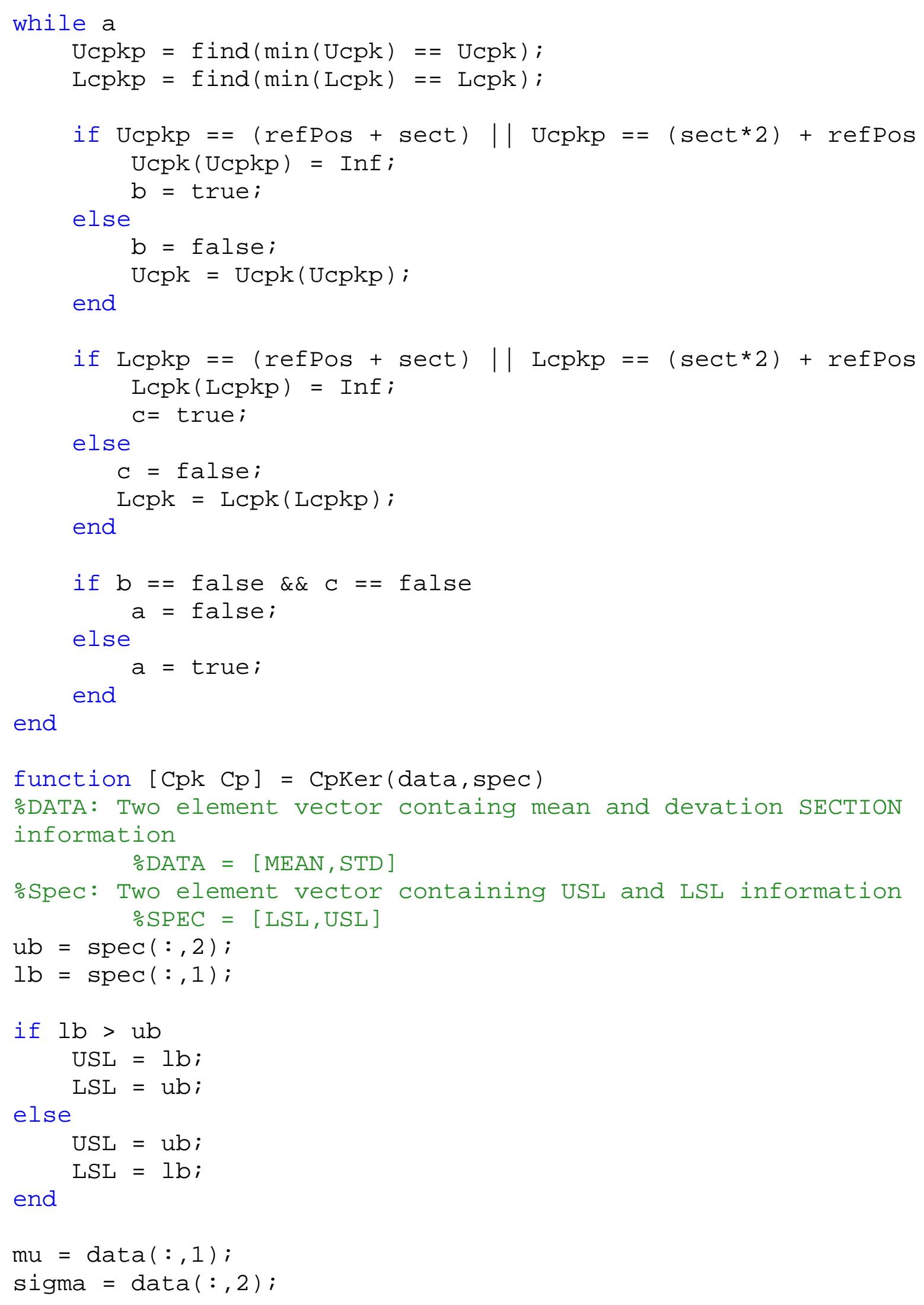


$\mathrm{a}=(\mathrm{USL}-\mathrm{LSL}) \cdot /\left(6 \cdot{ }^{*}\right.$ sigma $) ;$

$\mathrm{b}=\min \left(\left((\mathrm{USL}-\mathrm{mu}) \cdot /\left(3 \cdot{ }^{*}\right.\right.\right.$ sigma $\left.)\right),\left((\mathrm{mu}-\mathrm{LSL}) \cdot /\left(3 \cdot{ }^{*}\right.\right.$ sigma $\left.\left.)\right)\right)$;

$\mathrm{Cp}=\operatorname{round}(\mathrm{a} / .001) * .001$

$\mathrm{Cpk}=\operatorname{round}(\mathrm{b} / .01)^{*} .01$;

function NaOffset $=$ optimizer ()

global bladecount c_loss $r$ lot sectQ

global header sect closure Psim

disp('Please standby...work in progress...')

disp( ' ')

\%Read in and determine size of the raw data from the Excel

Spreadsheet

[num txt raw] = xlsread('test4.xls'); clear txt; \%Reading in excel file with raw data.

$\left[\begin{array}{ll}r & c\end{array}\right]=\operatorname{size}(r a w) ;$

lot $=\operatorname{raw}\{2,2\} ; \%$ Sets the lot name from the raw data

partFile $=\operatorname{raw}\{2,1\} ; \quad \%$ Finds what part is being estimated

\%Determine what sections are present and how many sections in total section $=\operatorname{raw}\{2,7\}$;

count $=1$;

sectQ $=[]$;

sectCount $=[]$;

for index $=3: r$

test $=\operatorname{raw}\{$ index, 7$\}$;

if strcmpi(test, section)

count $=$ count +1 ;

if index $==r$ sectQ $=[$ sectQ, section];

end sectCount $=$ [ sectCount, count];

elseif strcmpi(test, section)

sect $Q=[$ sectQ, section];

sectCount $=$ [sectCount, count $]$;

count $=1$;

section = test;

end

end

sect $=$ length $(\operatorname{sect} Q)$; 
bladecount $=(r-1) / \sec t$;

clear test count

\%Check to make sure there are an equal number of files present for each

\%blade.

for index $=2:$ length ( sectcount)

if $\operatorname{sectCount}($ index -1$) \sim=\operatorname{sectCount}($ index) error('ErrorTests:failTest', 'Check the number of files for each section, "re-crunch" and put \n into a new excel spreadsheet') break

end

end

run(partfile) \%Tolerance File

lot $=\operatorname{raw}\{2,2\}$

$[$ Naavg Nadev] $=\operatorname{staker} 2(\operatorname{num}(:, 5)$, sect $)$;

if -isempty (Pref)

posRef $=$ find $($ Pref $==$ sect $Q)$;

else

end

$\operatorname{posRef}=[] ;$

[Laavg Ladev] $=\operatorname{staker} 2(\operatorname{num}(:, 7), \sec t) ;$

$[$ Taavg Tadev $]=\operatorname{staker} 2(\operatorname{num}(:, 9)$, sect $)$;

normalizer $=$ Naavg $(1)$

$\mathrm{N} 1=\operatorname{num}(:, 5)-$ normalizer;

$\mathrm{N} 1$ avg = staker2(N1, sect);

LEA1 = num $(:, 7)$ - normalizer;

LEA1avg = staker2(LEA1, sect);

TEA1 = num $(:, 9)$ - normalizer;

TEA1avg = staker2(TEA1, sect $)$;

offset $=[-18: 3: 18]$;

NATcpk $=[]$;

$\mathrm{UCPK}=[]$;

LCPK $=[]$

UPOS $=[]$;

LPOS $=[]$;

for $i 1=1:$ length (offset)

Pn1 $=\operatorname{peenER}(N 1$, offset $(i 1))$;

PNavg = staker2 $($ Pn1, sect $)$;

Plea1 $=\operatorname{peenER}($ LEA1, offset $($ i1 $))$; 


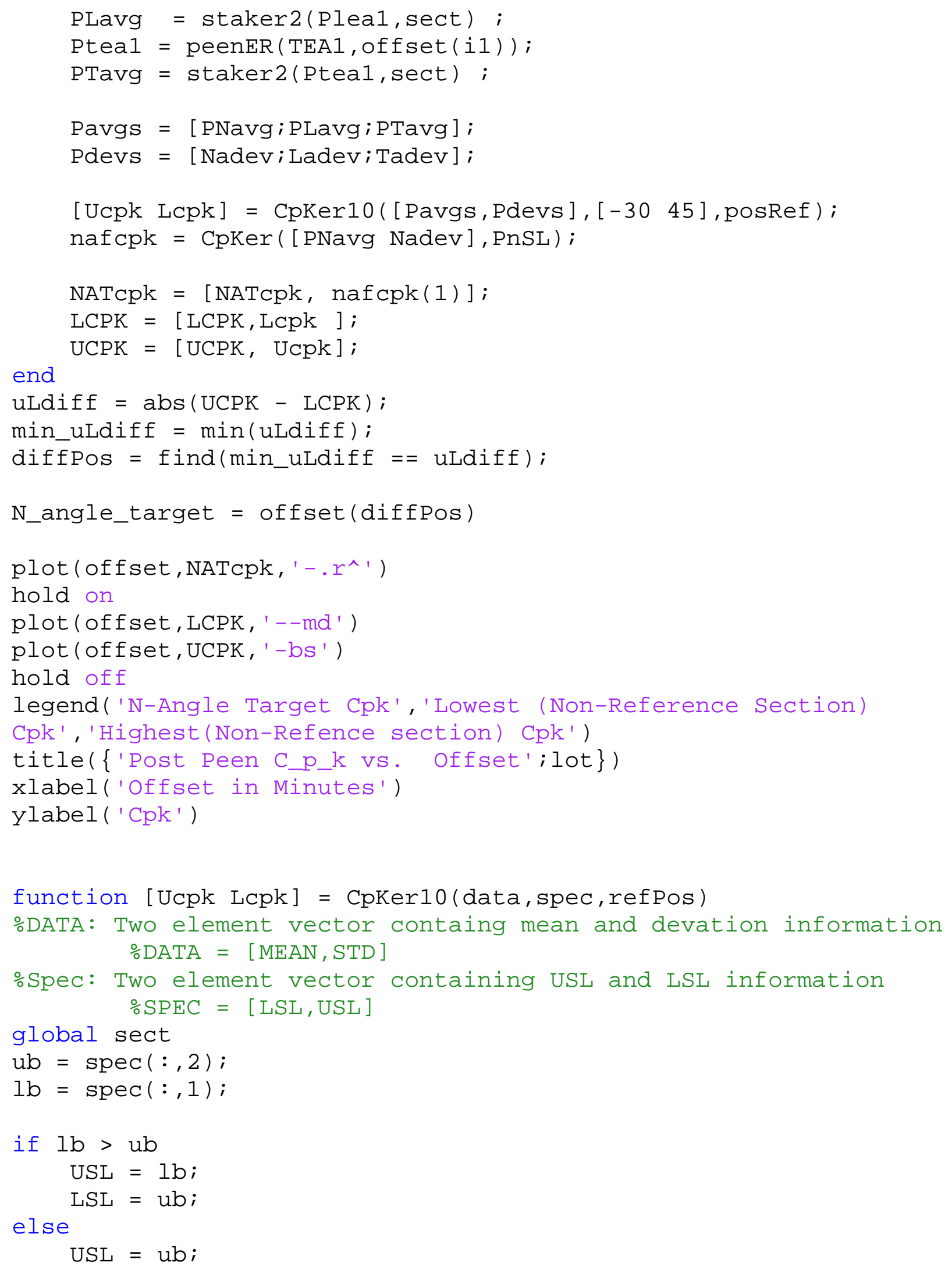




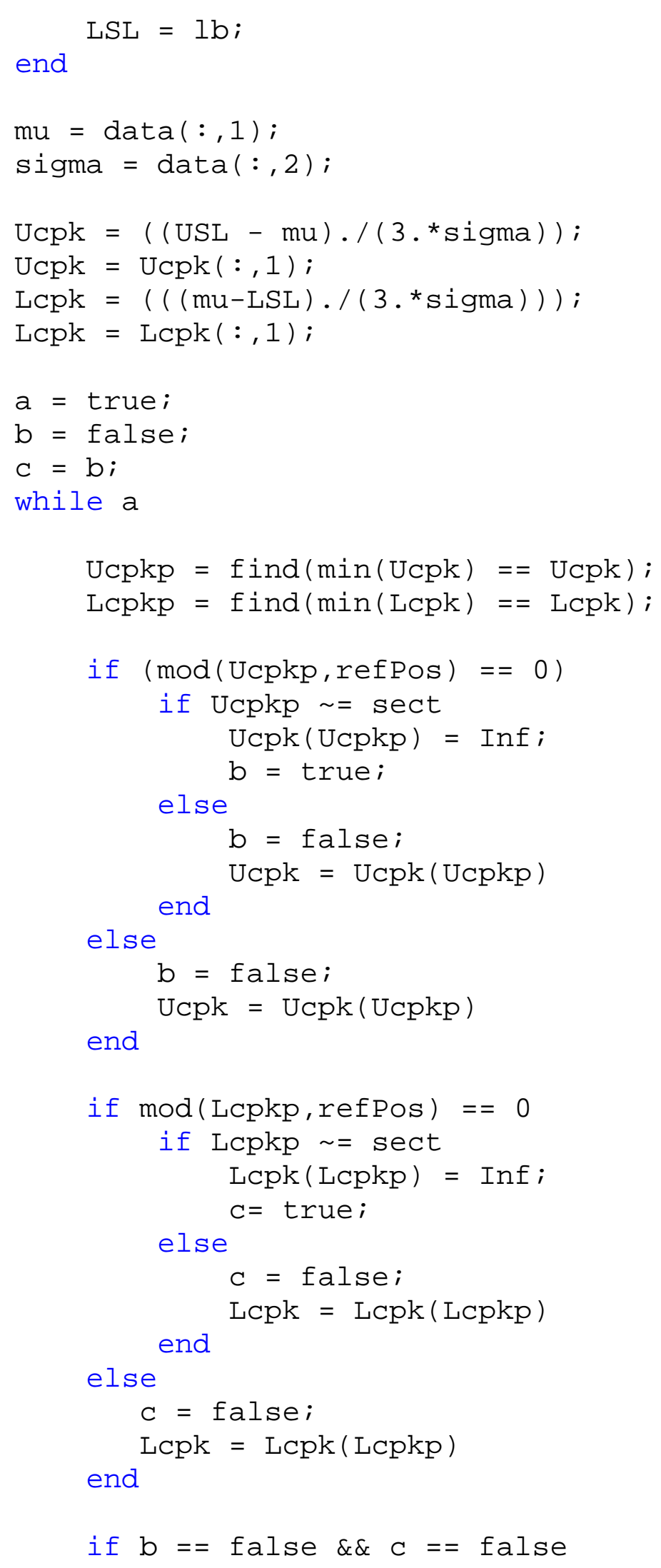




$$
\begin{aligned}
& \text { else }^{a}=\text { false } \\
& \text { end }
\end{aligned}
$$

end

function ret $=\operatorname{DTPer}$ (numMat)

global bladeCount sect\%Delta True Position Calculations.

$\% \mathrm{xxx}=\operatorname{numMat}(:, 1) ;$

$\%$ yyy $=\operatorname{nummat}(:, 2)$;

$\% \mathrm{n}=\operatorname{numMat}(:, 3)$;

$\%$ chord $=$ numMat $(:, 4)$;

$\% \operatorname{mxt}=\operatorname{numMat}(:, 5)$;

disp('Calculating Delta True Position for each blade...')

$c=$ zeros $\left(\right.$ bladecount $\left.{ }^{*} \sec t, 5\right)$;

$$
\begin{aligned}
& \text { for } i=1: \sec t-1 \\
& \text { if } i \sim=1 \\
& a=\text { numMat }\left(\left(\text { bladeCount }{ }^{*}(i-1)\right)+1: \text { bladeCount }{ }^{*} i,:\right) \text {; } \\
& b=\operatorname{numMat}\left(\left(\text { bladeCount }{ }^{*} i\right)+1:(\text { bladeCount } *(i+1)),:\right) \text {; } \\
& \text { else } \\
& c((b l a d e C o u n t *(i-1))+1:(b l a d e C o u n t * i),:)=b-a \text {; } \\
& \mathrm{a}=\operatorname{numMat}(1: \mathrm{bladeCount},:) ; \\
& \mathrm{b}=\text { numMat }\left(\text { bladeCount }+1: \text { bladeCount }{ }^{*} 2,:\right) \text {; } \\
& c(1: b l a d e C o u n t,:)=b-a \text {; } \\
& \text { end }
\end{aligned}
$$

end

ret $=c$;

function forecast(str)

close all

clc

global bladecount c_loss $r$ lot sectQ

global header sect closure Pref partFile

global Naoffset Psim Ftol Ptol

disp('Please select from the following: ')

disp('1 - Full Forecast (Lots over 100 pieces)')

disp('2 - Limited Forecast (Lots 100 pieces and less)')

choice $=$ input ( ' Please choose a number and press enter: ' $)$; clc 


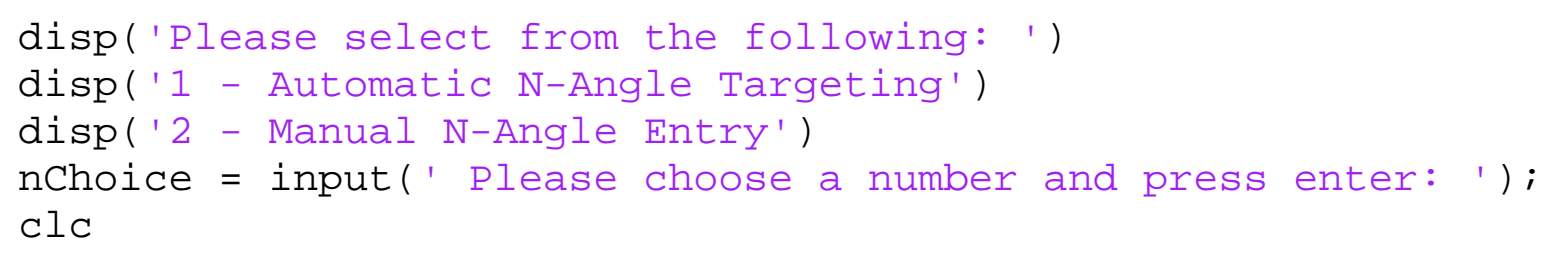

\%check to make sure there are an equal number of files present for each

\%blade.

for index $=2:$ length ( sectcount)

if $\operatorname{sectCount}($ index -1$) \sim=\operatorname{sectCount}($ index) 


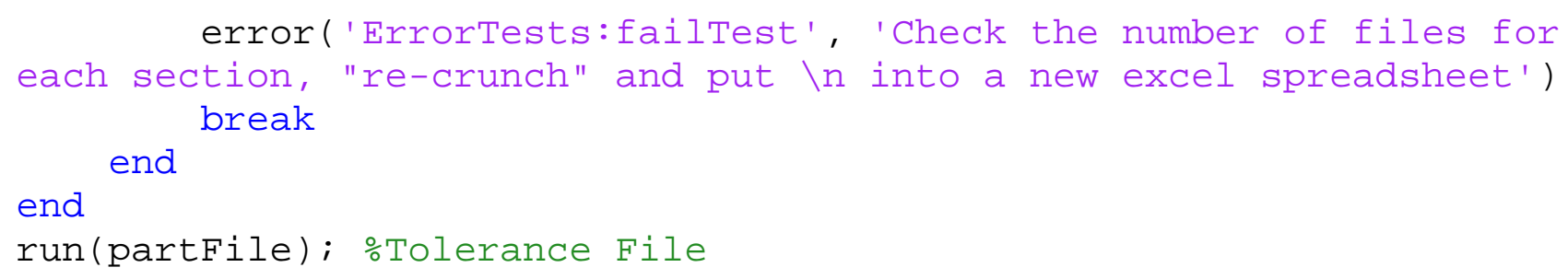


\%DTP

\%individual DTP

dtpMat $=\operatorname{DTPer}(\operatorname{num}(:,[2,3,5,4,12])) ;$ \%Output colums: X, Y, N, C, MXT

$\%$ Averages

$[\mathrm{dXavg} d X d e v]=\operatorname{staker} 2(\operatorname{dtpMat}(:, 1),(\operatorname{sect}-1)) ;$

$[$ dYavg dYdev $]=\operatorname{staker} 2(\operatorname{dtpMat}(:, 2),(\operatorname{sect}-1))$;

$[$ dNavg dNdev $]=\operatorname{staker} 2(\operatorname{dtpMat}(:, 3),(\operatorname{sect}-1))$;

$[$ acavg acdev $]=\operatorname{staker} 2(\operatorname{dtpMat}(:, 4),(\operatorname{sect}-1))$;

$[$ aMavg aMdev $]=\operatorname{staker} 2(\operatorname{dtpMat}(:, 5),(\operatorname{sect}-1))$;

$\operatorname{dtpMat}=\operatorname{dtpMat}(1:($ bladecount* $(\operatorname{sect}-1)),:) ; \quad \%$ reduces size of dtpMat to elimate unnecessary zeros.

deltaAvg $=[$ dXavg dYavg dNavg aCavg aMavg];

$\% \%$ cp/Cpk@IP:

$[\mathrm{xcpk}$ xсp $]=\operatorname{CpKer}([\mathrm{Xavg} X \mathrm{Xdev}], \mathrm{XSL})$;

$[$ ycpk ycp] $=\operatorname{CpKer}([$ Yavg Ydev $], Y S L)$;

$[$ ccpk ccp $]=\operatorname{cpKer}([$ Cavg Cdev], CSL $)$;

[nacpk nacp $]=\operatorname{CpKer}([\mathrm{N} 1$ avg Nadev $], \mathrm{NaSL}) ;$

[lacpk lacp $]=$ CpKer ([LEA1avg Ladev], LaSL);

$[$ tacpk tacp $]=\operatorname{CpKer}([$ TEA1avg Tadev $]$, TaSL $) ;$

[ltcpk ltcp $]=\operatorname{CpKer}([$ Ltavg Ltdev $]$, LETSL $) ;$

$[$ ttcpk ttcp $]=\operatorname{CpKer}([$ Ttavg Ttdev $]$, TETSL $)$;

$[\mathrm{mtcpk}$ mtcp $]=\operatorname{CpKer}([$ Mtavg Mtdev $], M X T S L)$;

cp_IP $=[x c p$ yсp cсp nacp lacp tacp ltcp ttcp mtcp];

cpk_IP $=[$ xcpk ycpk ccpk nacpk lacpk tacpk ltcpk ttcpk mtcpk];

IPpc $=[$ cp_IP; zeros $(1,9) ; c$ pk_IP $]$;

$\% \%$ Estimated Final Estimates \%\%

\%Chord @ Final:

[ cfAvg cfDev cfMat $]=\operatorname{chordCalcs}($ num $) ;$

\%LET, TET, MXT Final Calculation:

LET $=\operatorname{num}(:, 10)$;

LET_F = LET + etch;

Ltfavg = Ltavg + etch;

TET = num $(:, 11)$;

TET_F = TET + etch;

Ttfavg = Ttavg + etch;

MXT = num $(:, 12)$; 


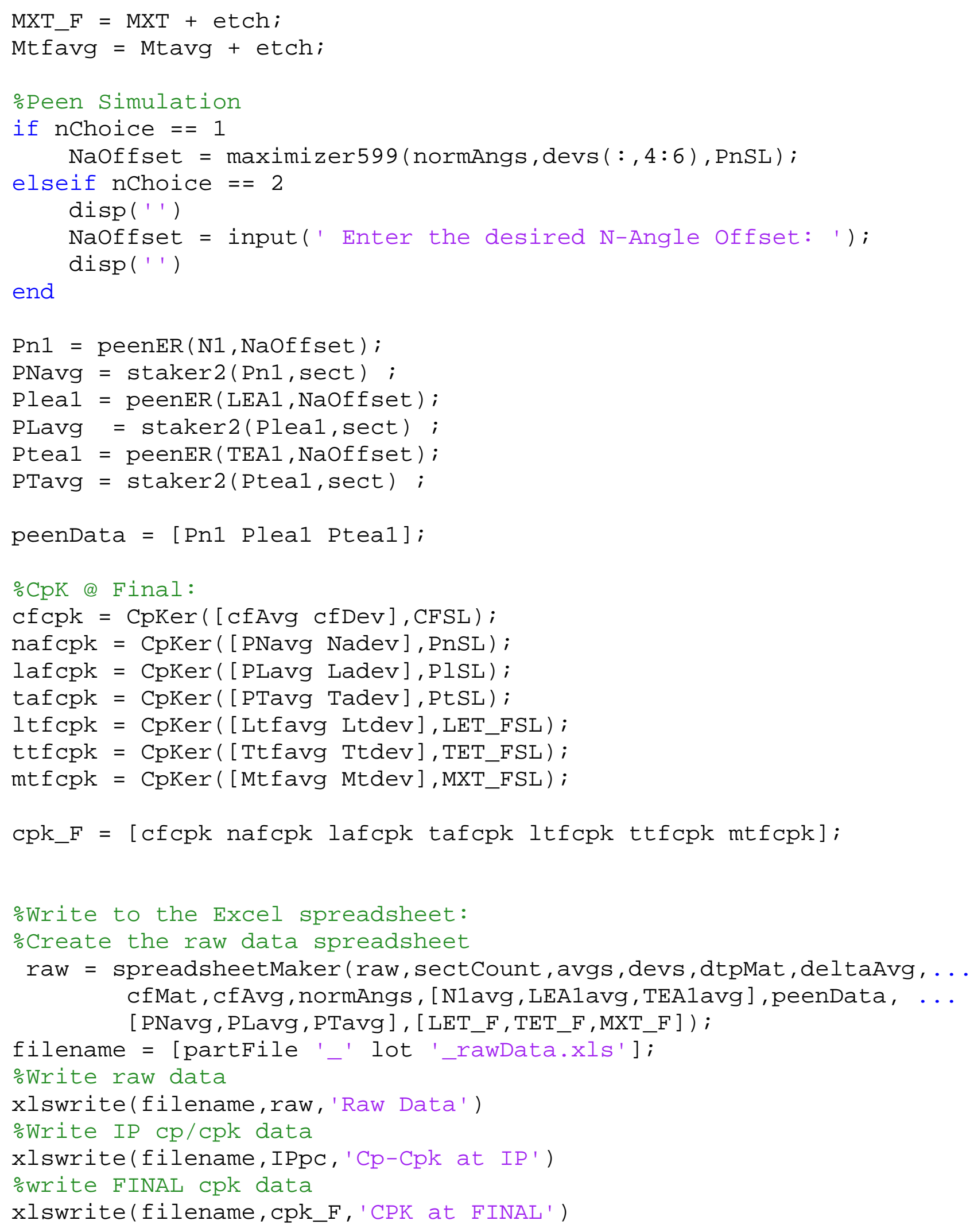




\section{\%Plot data}

\%create text for graphs

ref_onlyText = 'Information 0nly - not a product requirment'; if strcmp('A2JAK818', partFile) else sectTarget $=[$ 'center of tolerance at sect ' sectQ(2)]; end sectTarget $=[$ 'center of tolerance at Sect ' sectQ(1)];

AngleTargetText $=\left[{ }^{\prime} \mathrm{N}\right.$-Angle distribution adjusted to',$\ldots$ sectTarget];

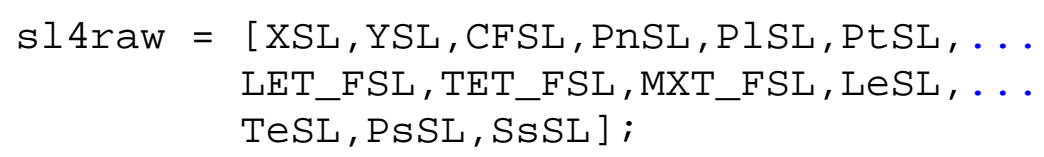




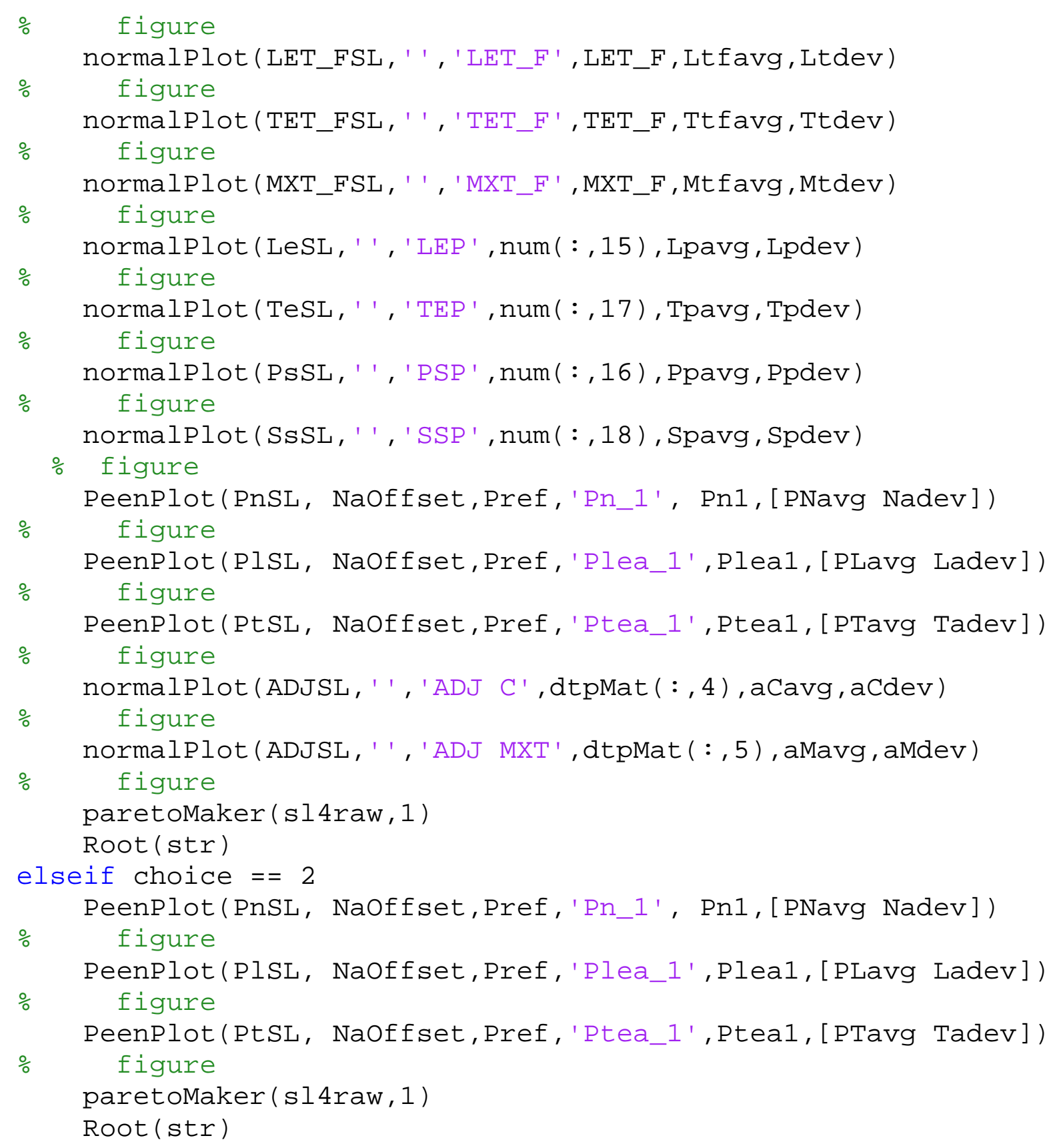

end

function NaOffset $=$ maximizer599 $($ data, dev, specs $)$

\%MAXIMIZER599

\%This function produces the optimal $\mathrm{N}$-Angle offset for this data set.

$\%$ Input the NORMALIZED N, LEA, TEA information into the DATA variable. $\%$ DATA $=[\mathrm{N}, \mathrm{LEA}, \mathrm{TEA}]$

$\% \mathrm{DEV}=[\mathrm{N}, \mathrm{LEA}, \mathrm{TEA}]<--* *$ Standard Deviation** 


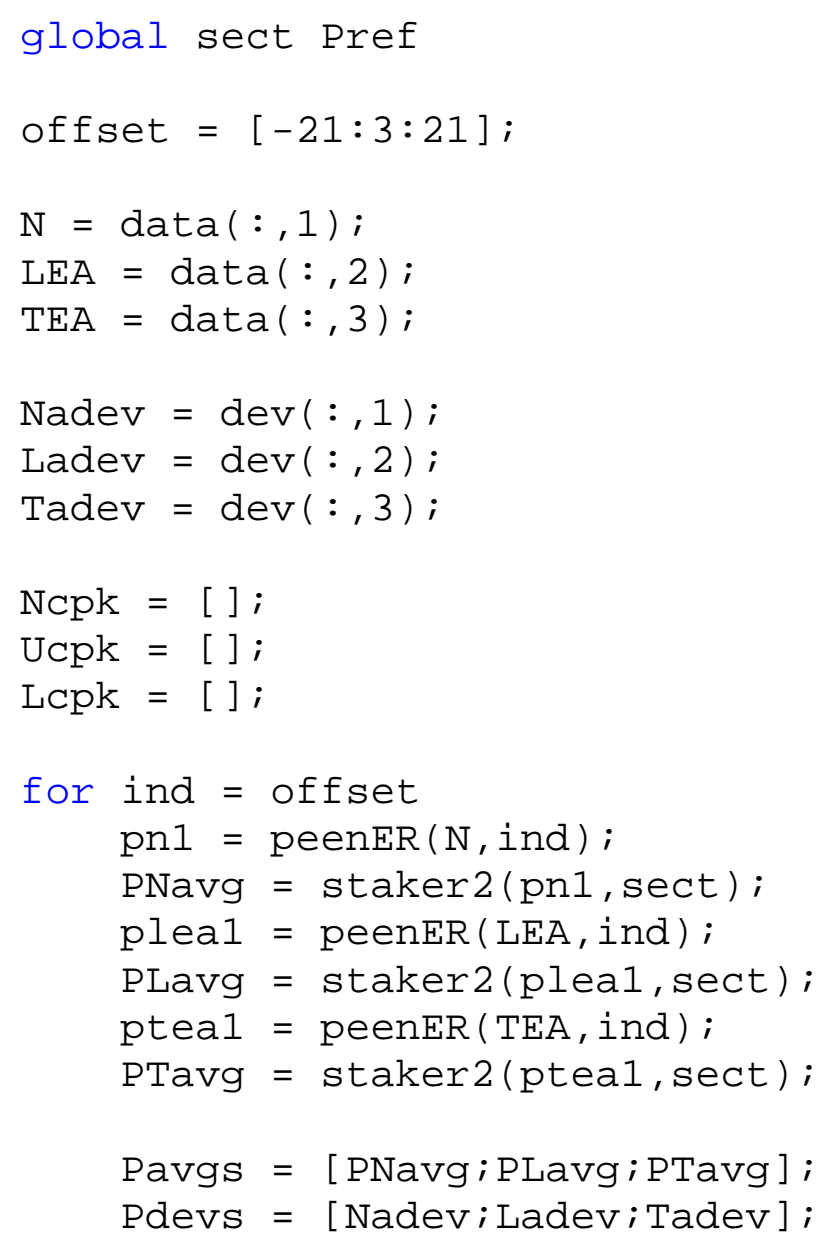

$[$ Uc LC $]=C p K e r 10([$ Pavgs, Pdevs $],[\operatorname{specs}(1,1), \operatorname{specs}(1,2)]$, Pref $) ;$

$$
\begin{aligned}
& \text { Ncpk }=[\text { Ncpk, CpKer }([\operatorname{PNavg}, \operatorname{Nadev}],[\operatorname{specs}(1,1), \operatorname{specs}(1,2)])] ; \\
& \text { Ucpk }=[\operatorname{Ucpk}, \operatorname{Uc}] ;
\end{aligned}
$$

end$$
\text { Lcpk = [Lcpk, Lc }] \text {; }
$$

plot (offset, Lcpk, offset, Ucpk)

ULdiff = abs (Ucpk - Lcpk);

maxYield $=\min ($ ULdiff);

NaOffset $=\operatorname{offset}($ find $(\operatorname{maxYield}==$ ULdiff $))$;

function normalplot (specs, ref, ptype, data, avg, dev, varargin) \%lot Forecasting Control chart. \%Creates a control chart given the LSL and USL as a two-element vector, SPECS, and the AVG and DEV vectors \%that are relvant to the feature being plotted. Also shades out any reference sections yellow that are \%in the REF variable found in the part file. 
global lot bladecount header sect sectQ partFile

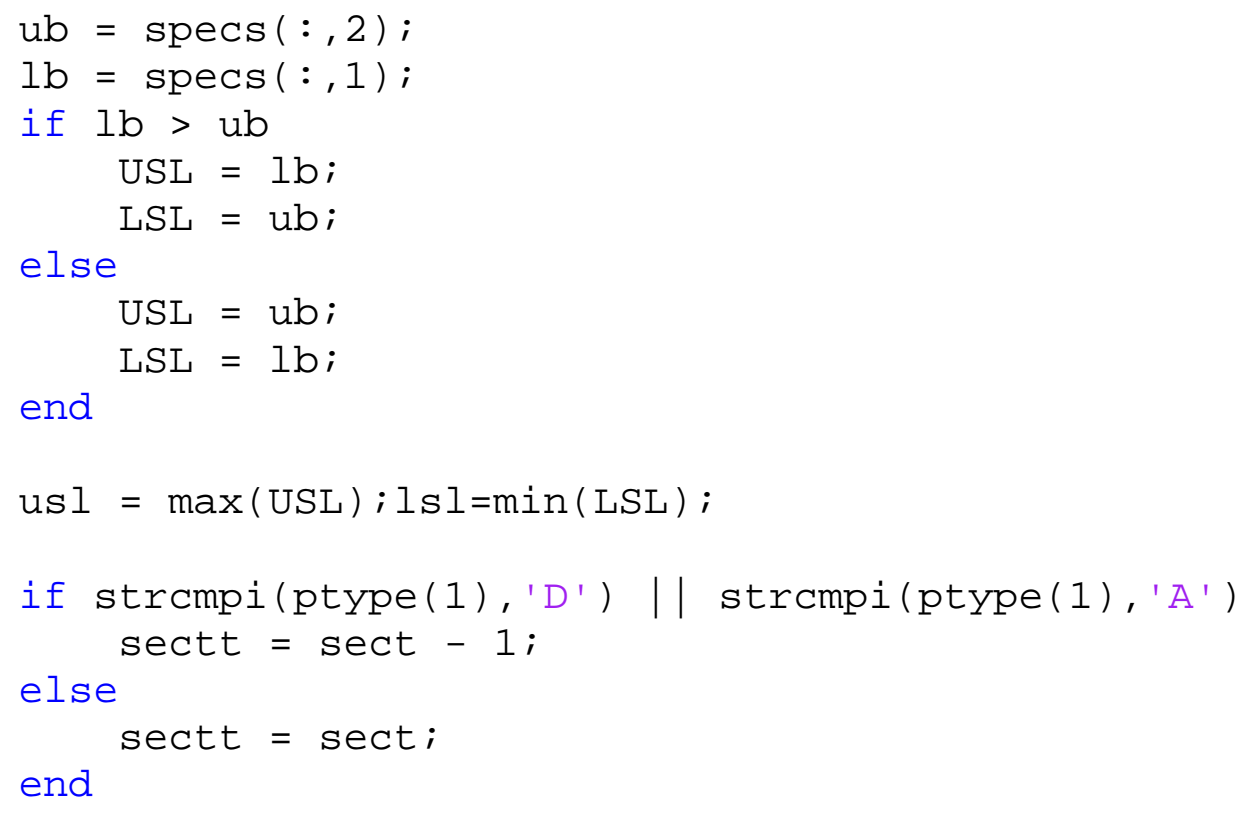




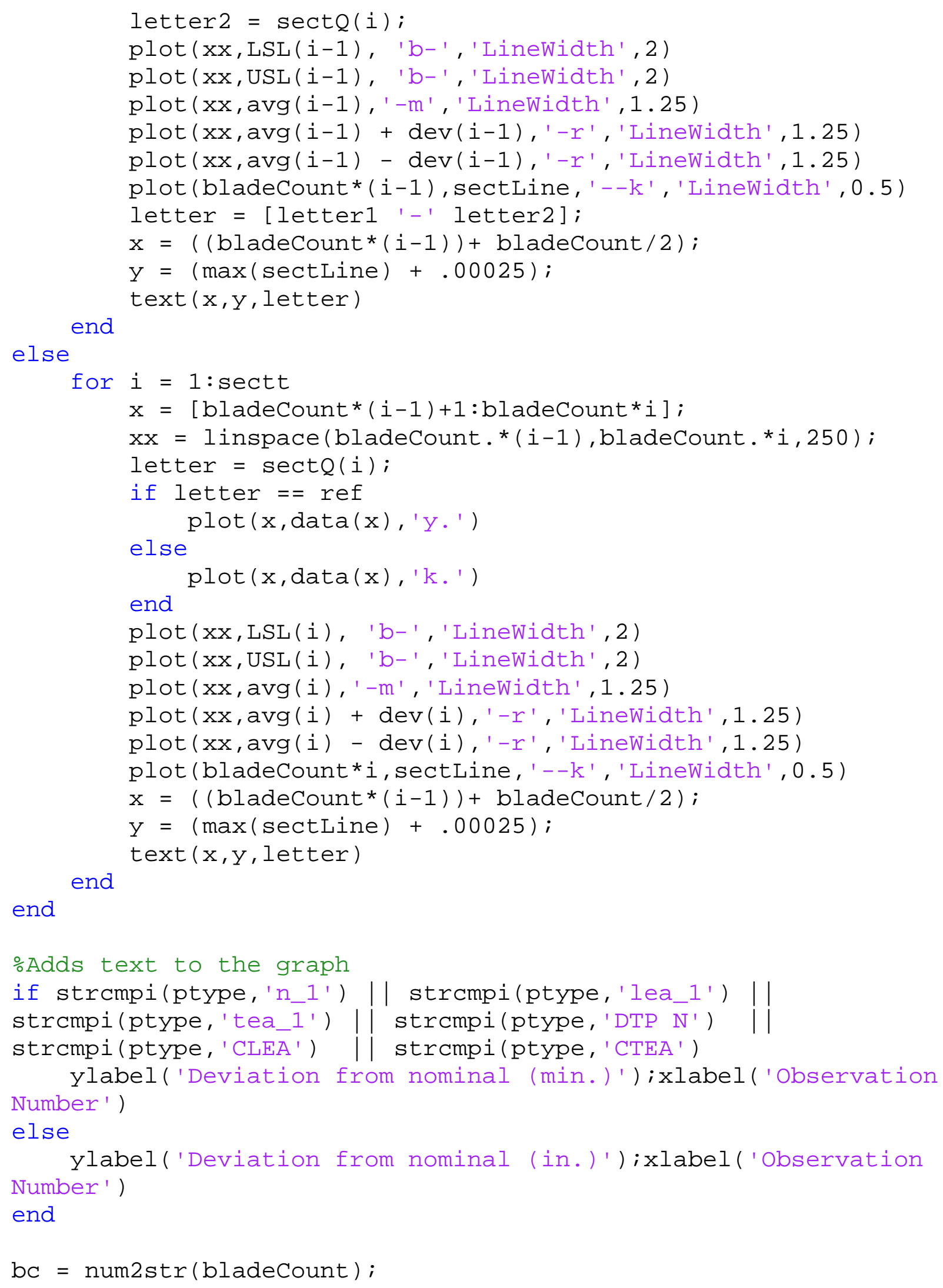




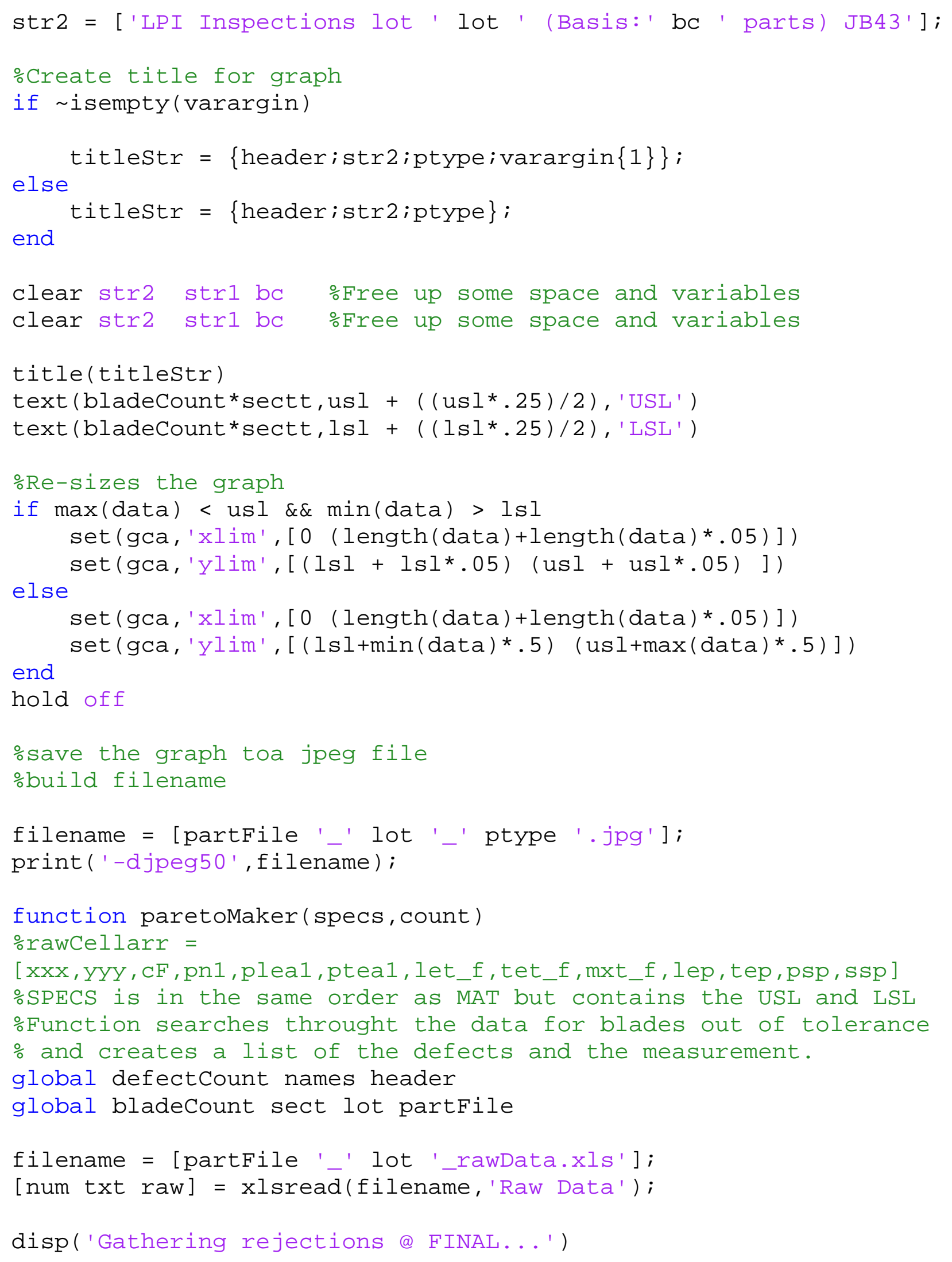




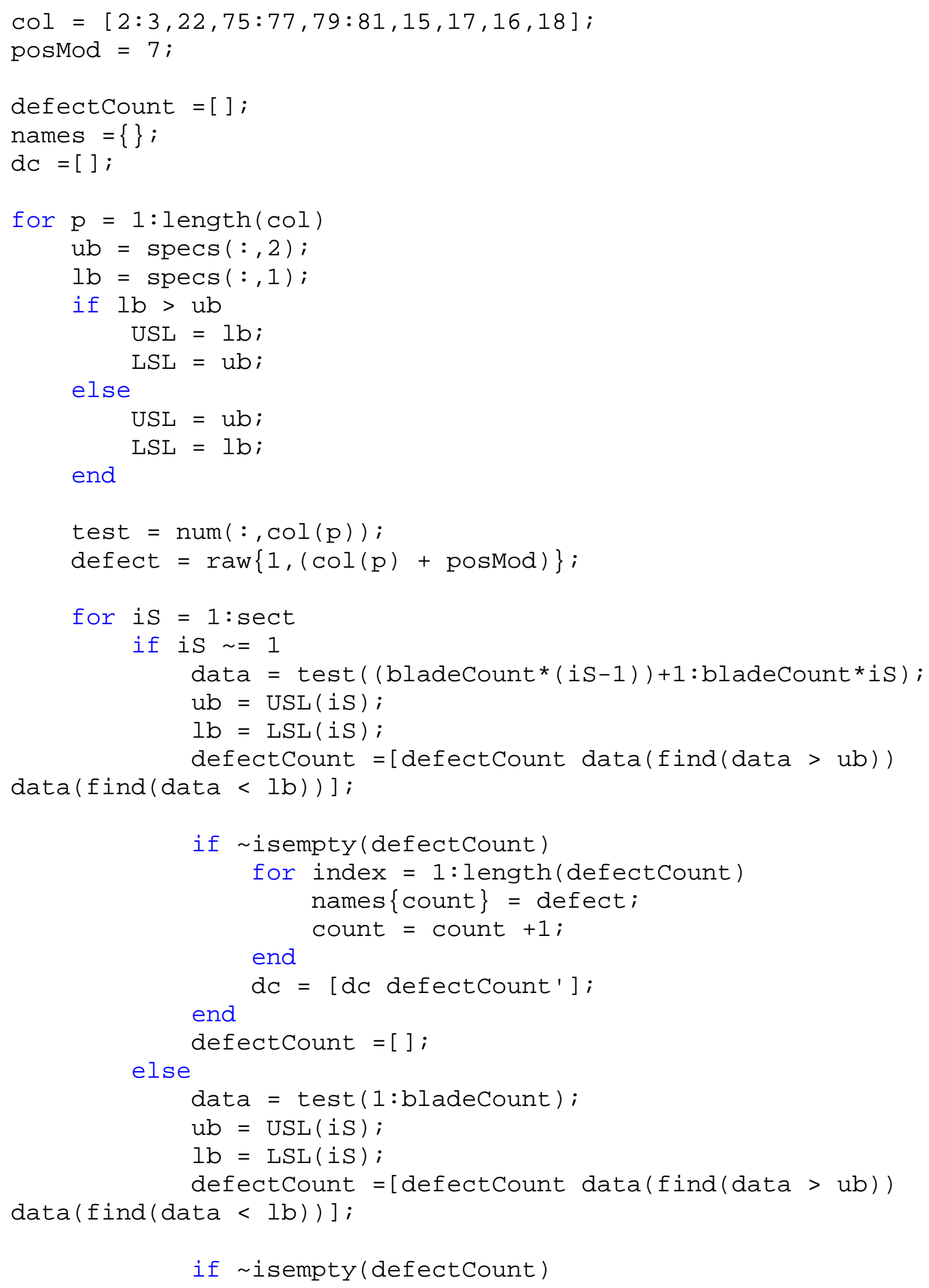




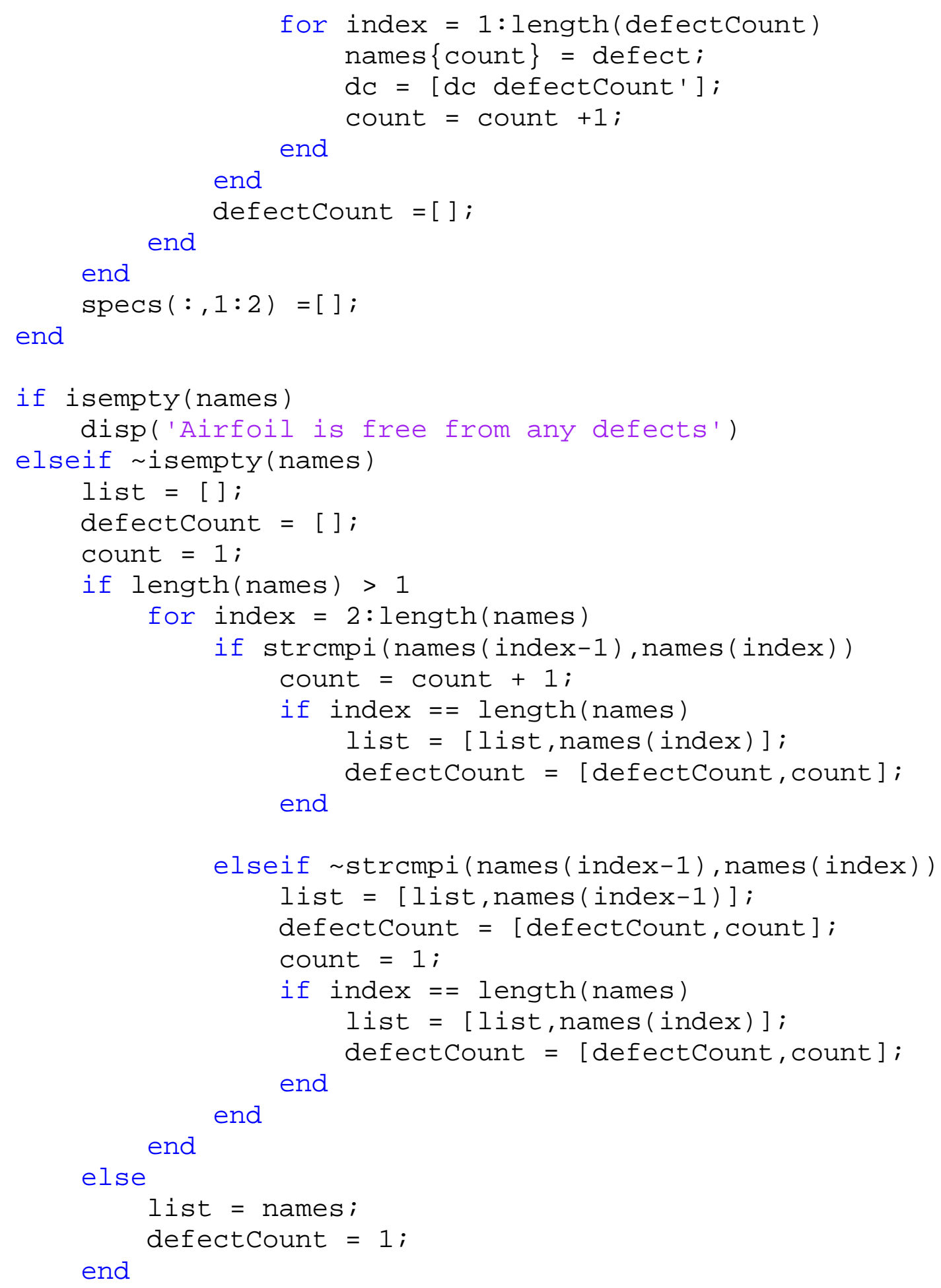




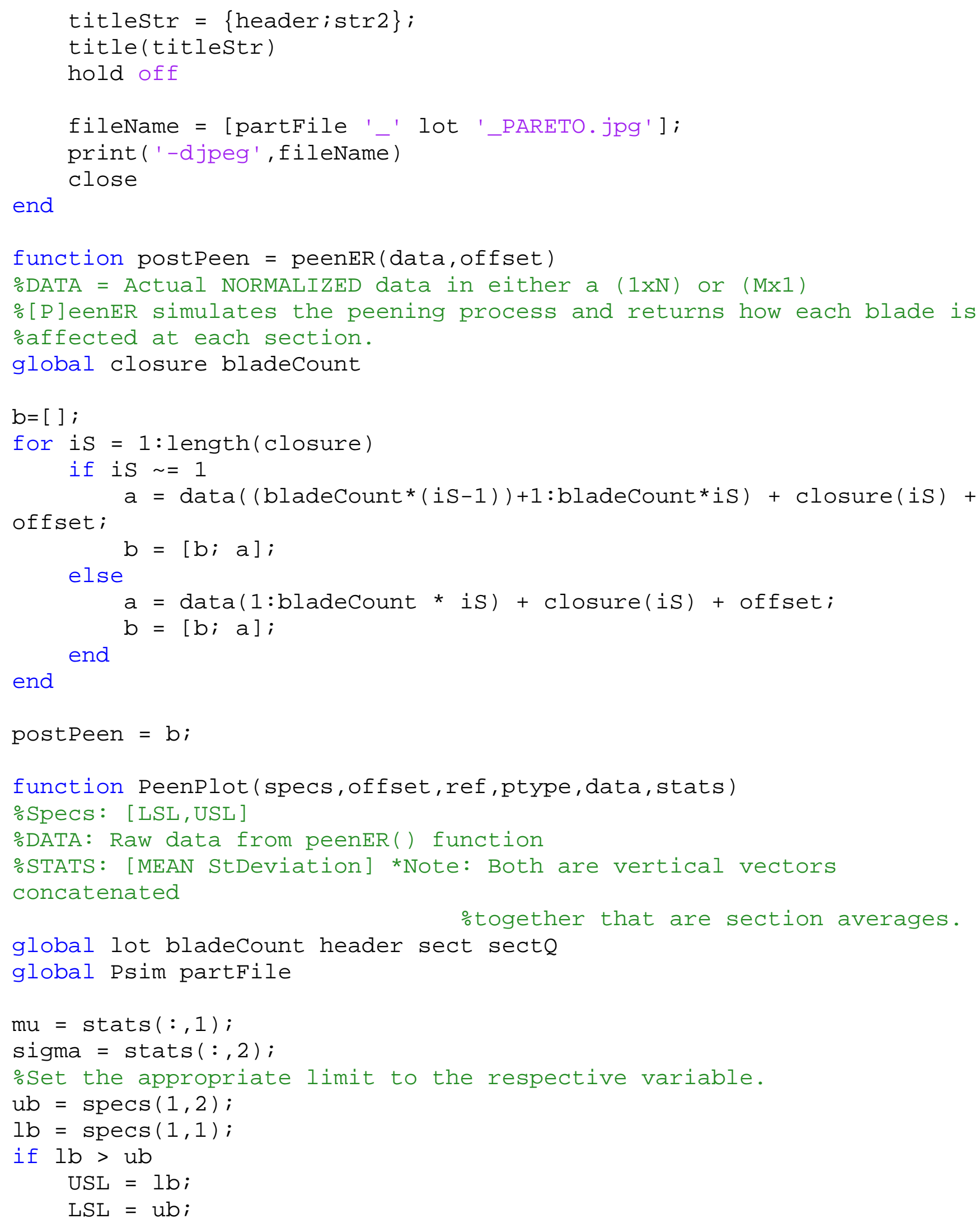




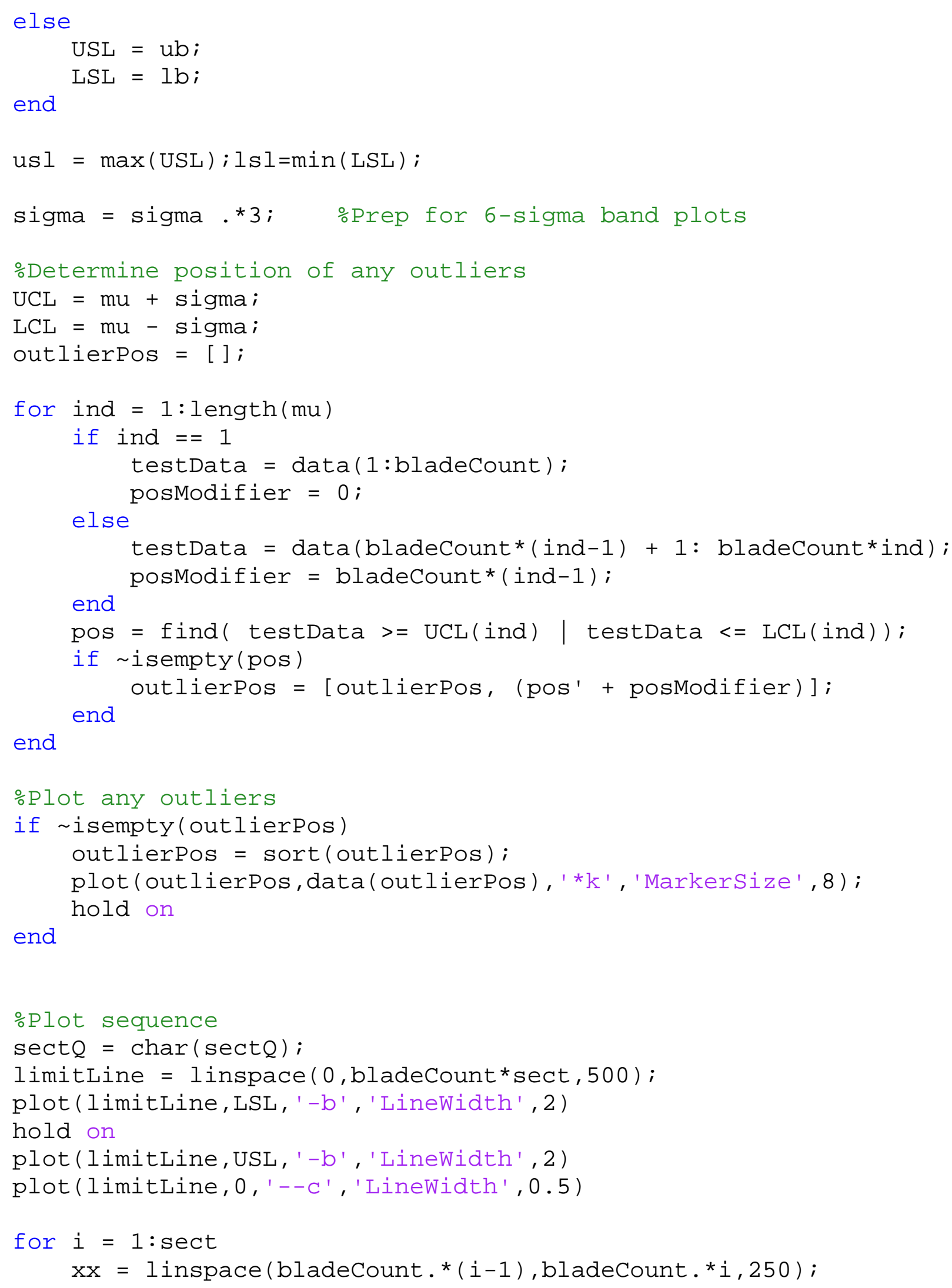




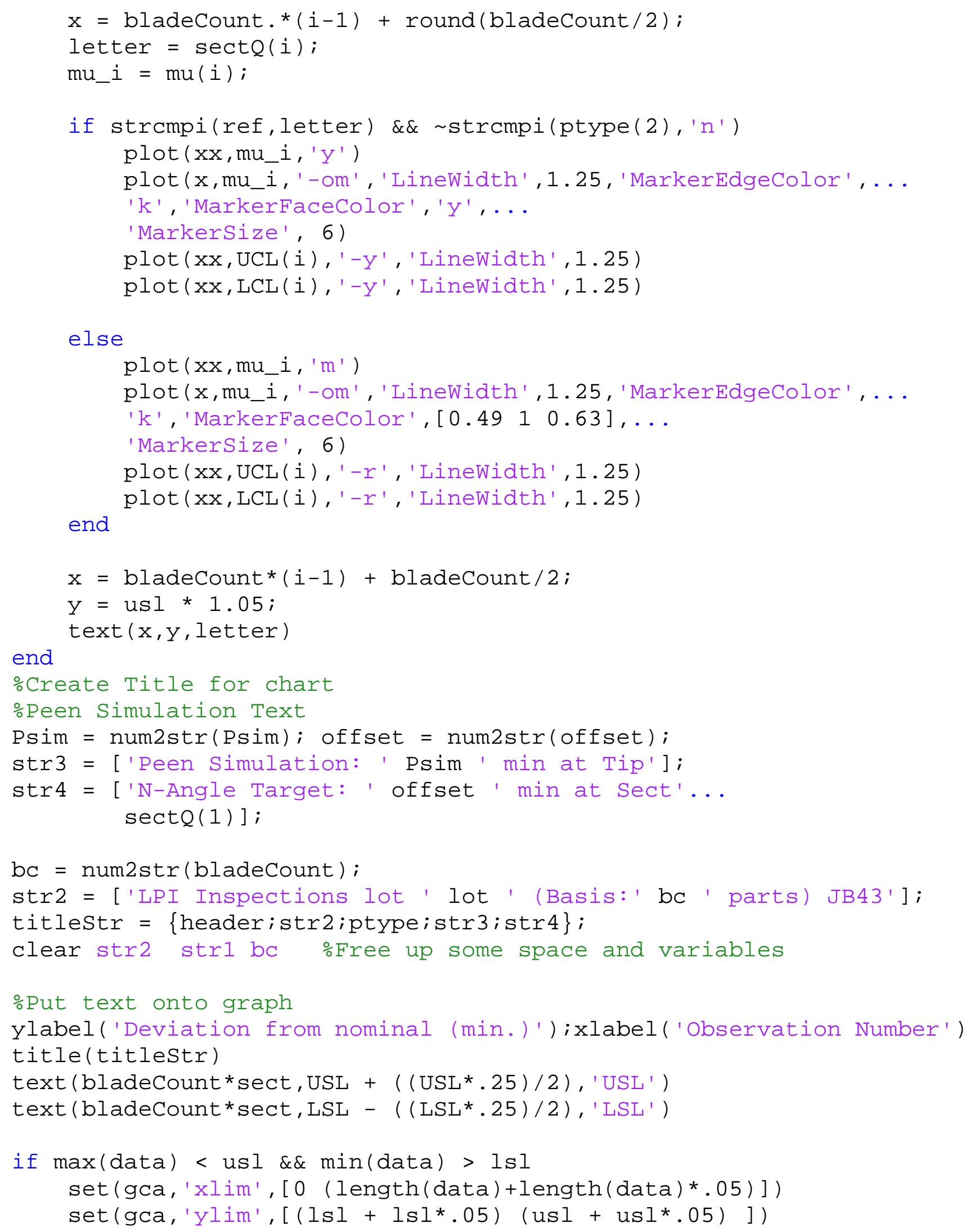




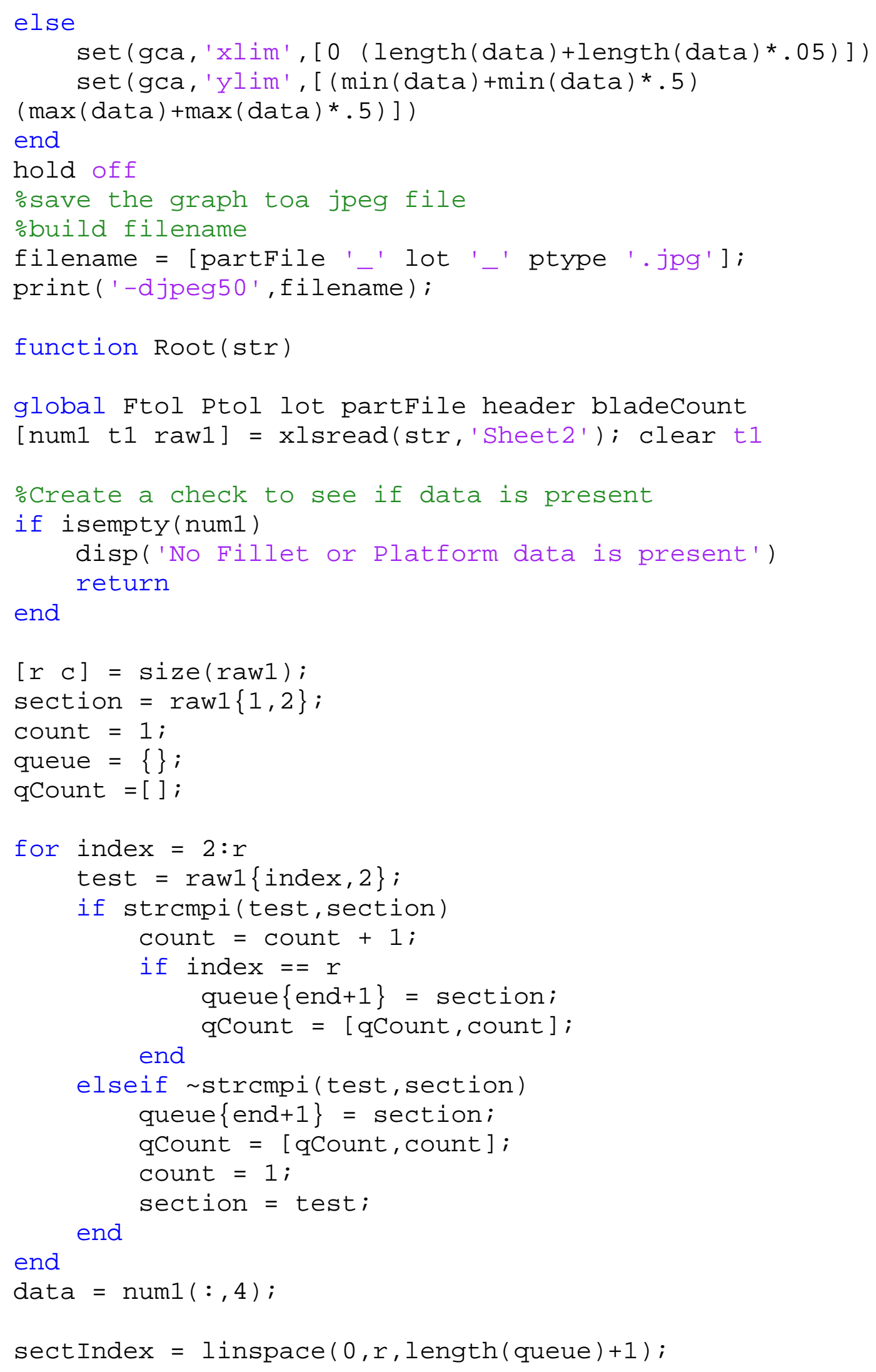




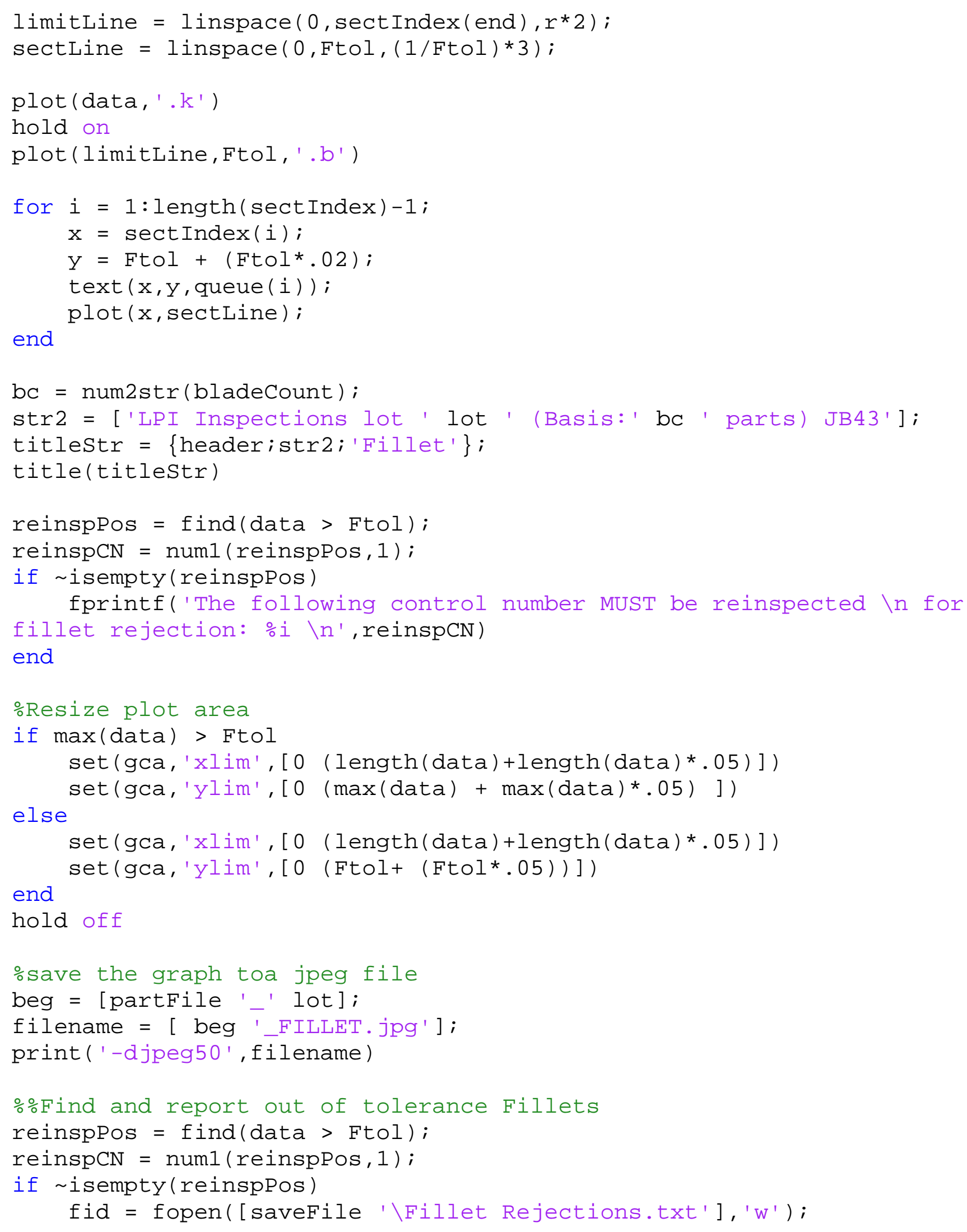




\section{for $i=1:$ length (reinspPos)}

fprintf(fid, 'The following control number is out of tolerance

for the FILLET feature: \%i $\backslash n^{\prime}$, reinspCN(i));

end

fclose(fid);

end

[num2 t2 raw2] = xlsread(str,'Sheet3'); clear t2

$[r \quad c]=\operatorname{size}($ raw2 $)$

section $=\operatorname{raw} 2\{1,2\}$;

count $=1$;

queue $=\{\}$;

qCount $=[]$;

for index $=2: r$

test $=\operatorname{raw} 2\{$ index, 2$\}$;

if strcmpi(test, section)

count $=$ count +1 ;

if index $==r$

queue $\{$ end +1$\}=$ section;

end

qCount $=$ [qCount, count $]$;

elseif strcmpi(test, section)

queue $\{$ end +1$\}=$ section;

qCount $=$ [qCount, count $]$;

count $=1$;

section = test;

end

end

data $=\operatorname{num} 2(:, 5)$;

sectIndex $=$ linspace $(\Theta, r$, length (queue $)+1)$;

limitLine $=$ linspace $(\Theta$, sectIndex $($ end $), r * 2)$;

sectLine $=$ linspace $(\operatorname{Ptol}(1), \operatorname{Ptol}(2),(1 / \operatorname{Ptol}(2)) * 3)$;

plot (data, '. k')

hold on

plot (limitLine, Ptol(1), ' - b')

plot (limitLine, Ptol(2), ' - b')

for $i=1$ : length ( $\operatorname{sect}$ Index $)-1$;

$x=\operatorname{sectIndex}(i)$;

$y=\operatorname{Ptol}(2)+(\operatorname{Ptol}(2) * .02)$;

sectText = queue $(i)$;

text $(x, y$, sectText $)$;

$\operatorname{plot}(x$, sectLine); 
end

titlestr $=\{$ header;str2;'Platform' $\}$;

title(titlestr)

\%Resize plot area

if $\max ($ data $)<\operatorname{Ptol}(2)$ \&\& $\min ($ data $)>\operatorname{Ptol}(1)$

set (gca, 'xlim', [0 (length(data)+length(data)*.05)] )

]) set (gca, 'ylim', [(Ptol(1)+Ptol(1)*.05) (Ptol(2) +Ptol(2)*.05)

else

set (gca, 'xlim', [0 (length(data)+length(data)*.05)] )

set $($ gca, 'ylim', $[(\operatorname{Ptol}(1)+\min ($ data $) * 5)(P t o l(2)+$

$\left.\left.\max (\text { data })^{*} .5\right)\right]$ )

end

hold off

\%save the graph toa jpeg file

\%build filename

filename = [ beg '_PLATFORM.jpg'];

print ('-djpeg50', filename)

reinspPos $=$ find $($ data $>$ Ptol(2) $\mid$ data $<$ Ptol(1));

reinspCN = num2 (reinspPos, 1$)$;

if -isempty (reinspPos)

fid = fopen([saveFile '\Platform Rejections.txt'],'W');

for $i=1$ : length (reinspPos)

fprintf(fid, 'The following control number is out of tolerance

for the PLATFORM feature: \%i \n', reinspCN(i));

end

end

fclose(fid);

function

ret=spreadsheetMaker ( raw, scounter, avg, dev, dtpMat, deltaAvg, chordFinal, cFavg, normAngs, normAngsMean, pangs, pangsMean, thickness)

$\%$ Takes in the data matrices and places them in a cell array that is ready

$\%$ to be written to an Excel file.

global sect sectQ closure c_loss NaOffset

format bank

offset = NaOffset;

$\operatorname{raw}\{1$, end +2$\}=$ 'Sect' ;

for index $=1: \operatorname{sect}$

$\operatorname{raw}\{$ index +1 , end $\}=\operatorname{sectQ}($ index $) ;$ 


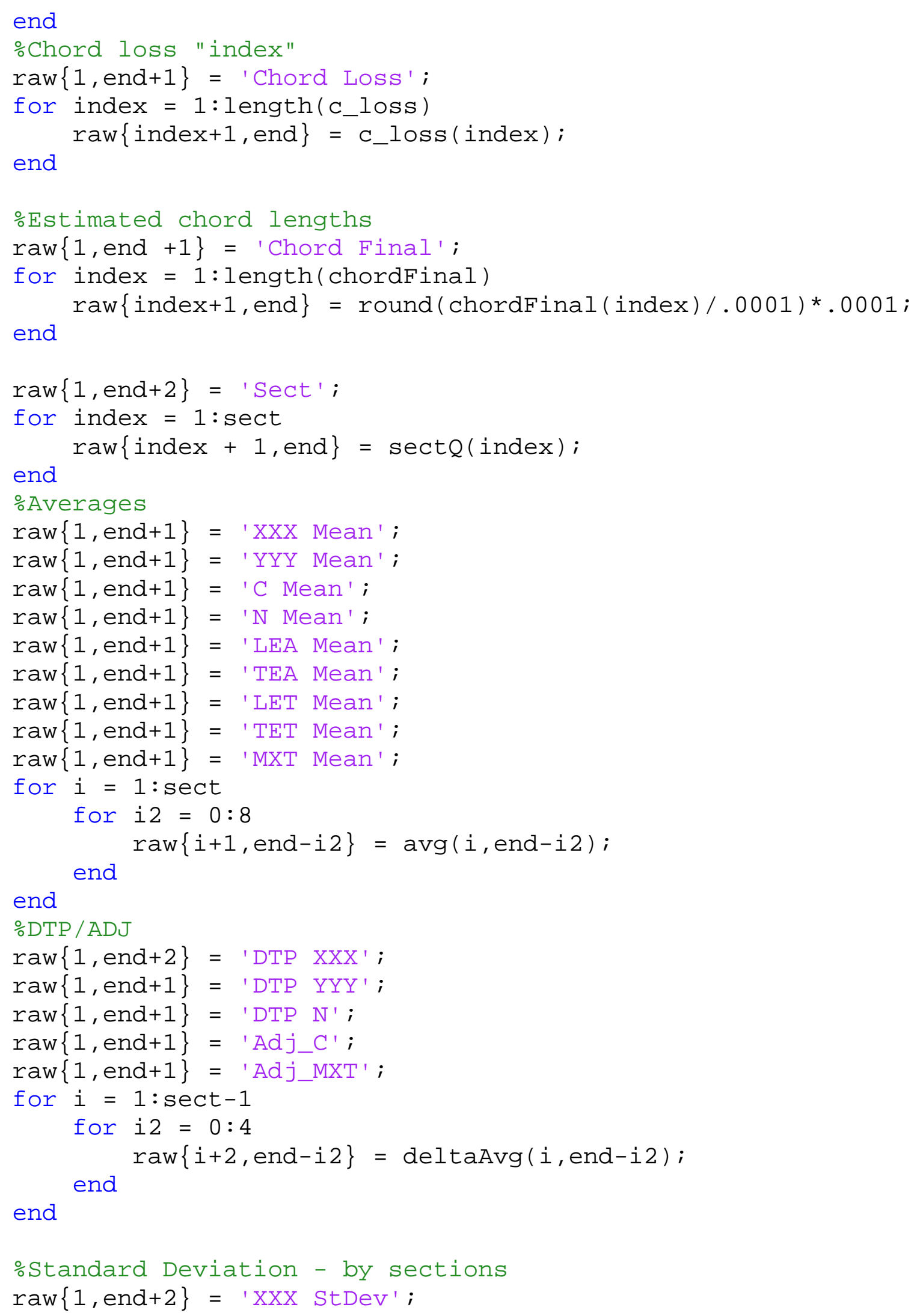




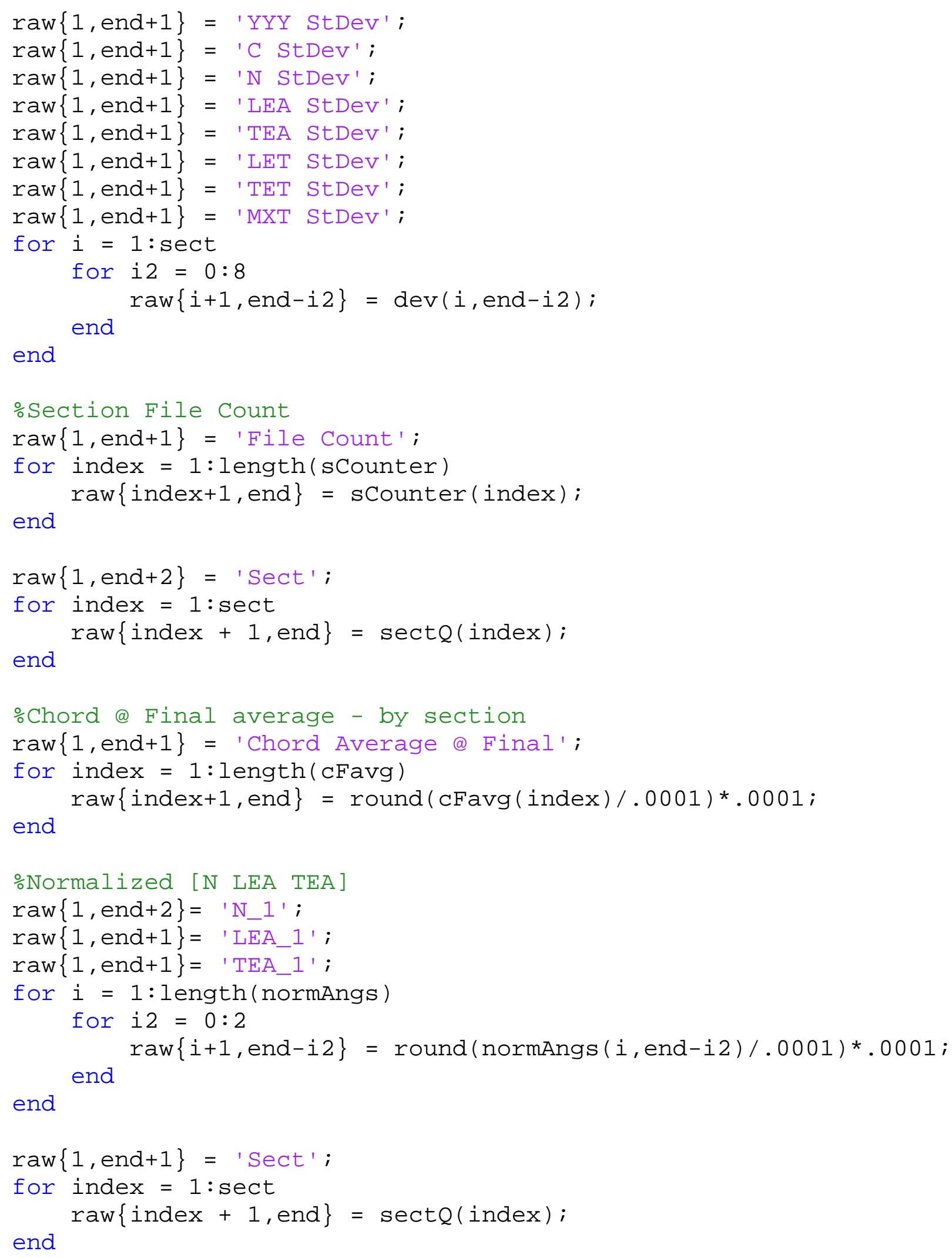




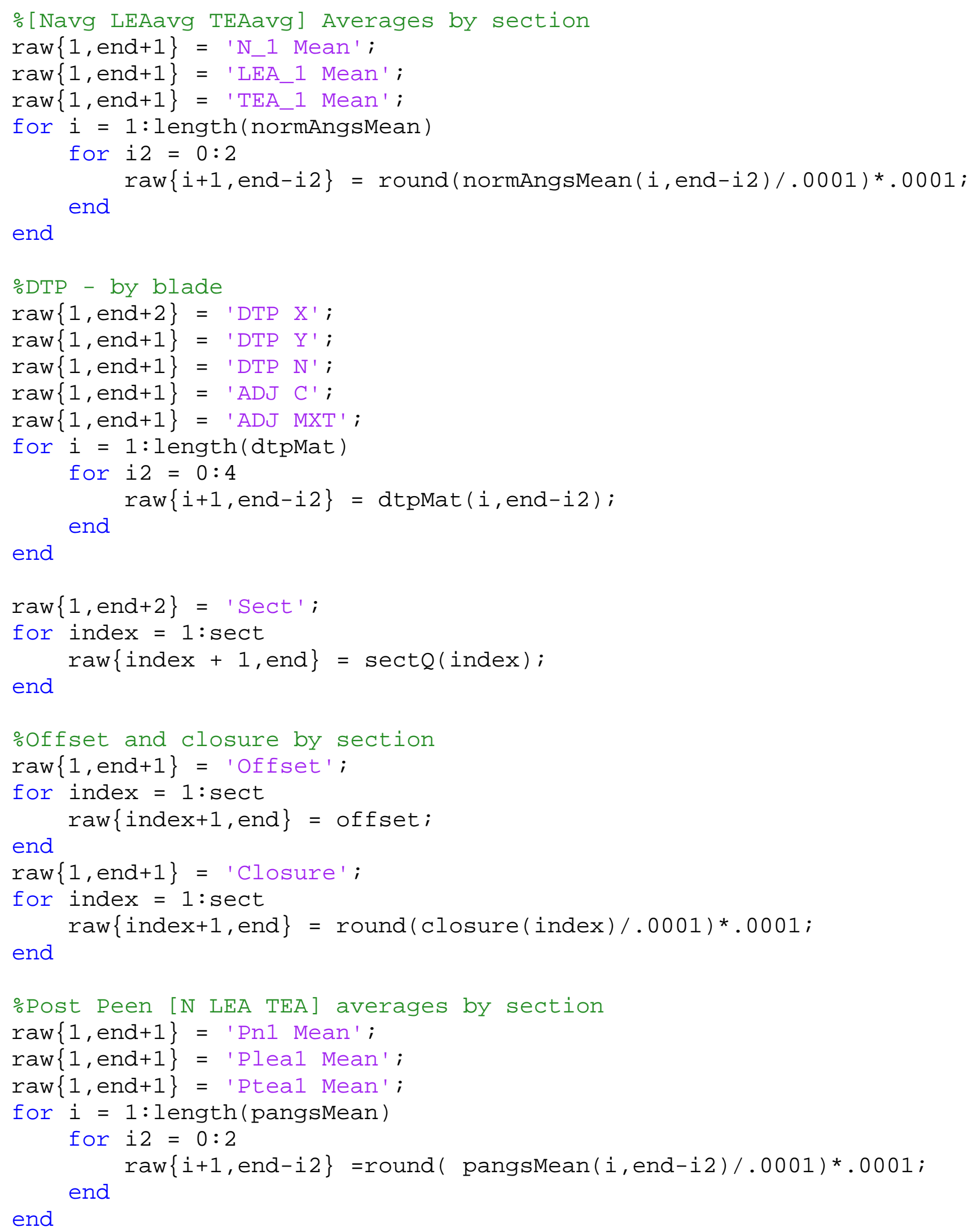




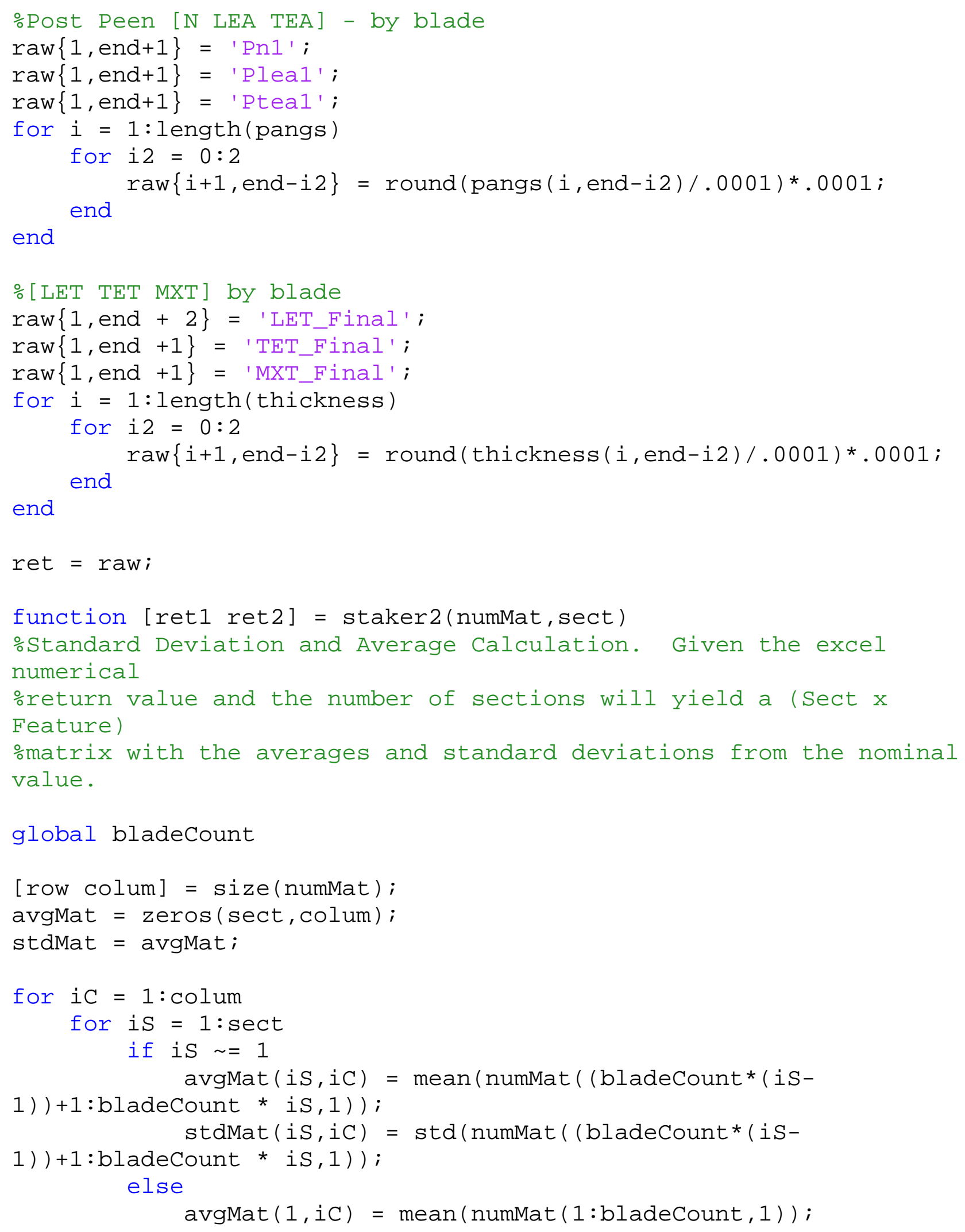




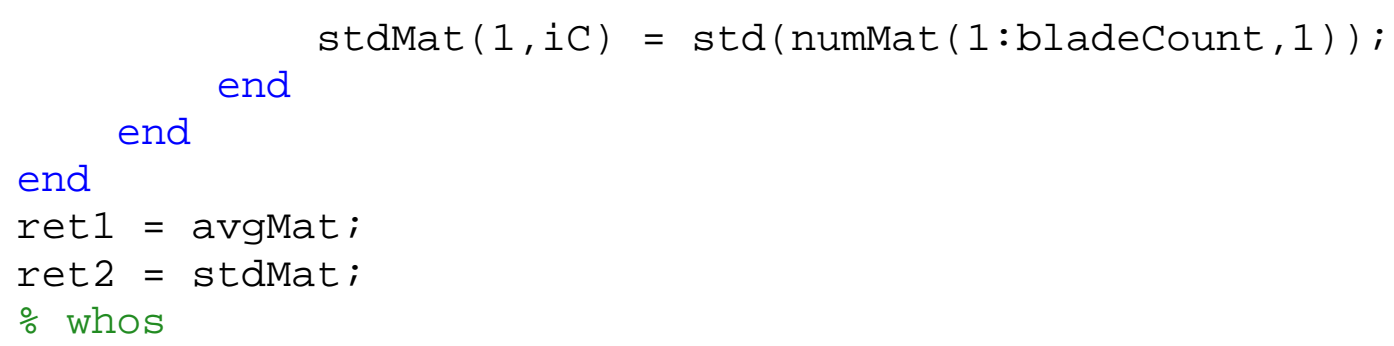

